

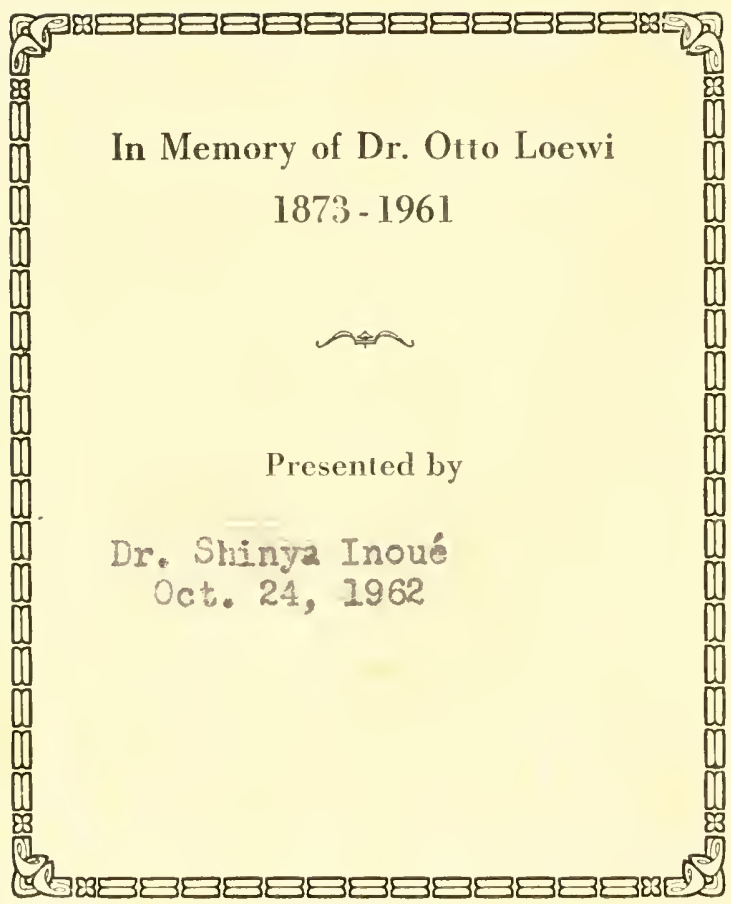




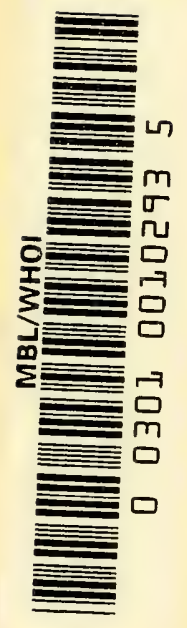




INTERNATIONAL SERIES OF MONOGRAPHS ON
PURE AND APPLIED BIOLOGY

Division: MODERN TRENDS IN PHYSIOLOGICAL SCIENCES

General Editors: P. Alexander and Z. M. Bace

Volume 9

PROGRESS IN MICROSCOPY 



\title{
PROGRESS IN MICROSCOPY
}

\author{
by \\ M. FRANÇON \\ PROFESSOR OF PHYSICS \\ THE SORBONNE, PARIS UNIVERSITY \\ AND THE INSTITUTE OF OPTICS
}

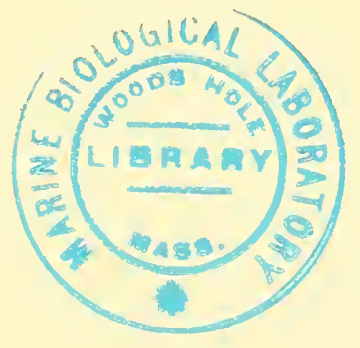

ROW. PETERSON AND COMPANY

Evanston, Illinois Elmsford, New York 
Not to be sold outside the

United States of America

or Canada

Copyright

(C)

1961

PERGAMON PRESS LTD

AMERICAN EDITION 1961

under license from

Pergamon Press Ltd

Library of Congress Card No. 61-10645

Printed in Poland to the order of Panstwowe Wrdawnictwo Namkone by Drukarmia Uniwersytetn Jagielloniskiego, Cracow 


\section{Contents}

FOREWORD

\section{Chapter I. Physical Aspects of Image Formation in Microscopy}

1. Introduction

2. Image of a luminous point exhibited by the objective of a microscope in monochromatic light

3. Image of a luminous point originated by a reflecting objective

4. Image of a luminous point when the microscope is not properly focused (monochromatic light)

5. Image of a luminous point in white light

6. Luminous point imaged in the presence of aberrations

7. Objects in coherent, incoherent and partially coherent illumination

8. Extended objects imaged in incoherent illumination

9. Extended objects imaged in coherent and partially coherent illumination

10. Perception limit and resolving power

11. Stray light

12. Reducing the grain of photographic images

\section{Chapter II. Phase-contrast Microscopy}

1. Transparent objects

2. Principle of the phase-contrast method

3. Contrast and sensitiveness of the method

4. Image structure in phase-contrast

5. Perception limit and resolving power. Effects of stray light

6. Phase-contrast microscopes in transmitted light

7. Phase-contrast applications

\section{Chapter III. Interference Microscopy in Transmitted Light}

1. Object and fundamentals of interierence microscopy 94

2. Fundamentals of two-wave interference microscopes 94

3. Dyson's interference microscope 97

4. Leitz's interference microscope 101

5. Polarization interference microscopes 103

6. Theoretical principle of polarizing interference microscopes 104

7. Smith's full image duplication interference microscope 110

8. Françon's compensated interference eyepiece 113

9. L. P. Johansson's interferometer eyepiece 117

10. Nomarski's interference microscope 118

11. Axial image duplication interference microscopes 120

12. Multi-wave interference microscopes 122

13. Menzel's interference microscope 127

$\begin{array}{ll}\text { 14. Sensitivity of interference microscopes } & 128\end{array}$ 


\section{CHAPTEK IV. REFLECTED-LIGHT MiCROSCOPY}

1. Methods applied in reflected-light microscopy

2. Reflected-light phase-contrast. Jupnik, Osterberg and Pride phase microscope. Françon, Nomarski, Bendford Seidenberg layouts

3. Oblique illumination and dark ground in reflected light. Schmaltz's slit, Menzel's method

4. Reflected-light interference microscopes: Sagnac, Linnik-Zeiss, Krug and Lau, Hilger and Watt, Baker, Mirau, Dyson, Françon, Nomarski. Multi-wave layouts

5. Observation in polarized light

6. Scrutinizing of hot-metal surfaces

\section{Chapter V. Geometrical Measurements}

1. Settings in microscopy

2. Setting accuracy

3. Measuring a length in a focusing plane by means of two transverse settings 167

4. Measuring a thin object by two axial settings 168

5. Measuring a length whose ends do not lie in the same focusing plane 168

6. Measuring small areas and volumes

7. Determining the normal in a point of a small reflecting surface

8. High precision-measurement microscopes

Chapter Vi. Measuring Refraction indices of Transparent Objects:

Unpolarized Two-wave Interference Microscopes

1. Measuring path differences

2. Determining the refraction index from path difference measurement

\section{Chapter ViI. Measuring Refraction indices of Transparent Objects:} POLARIZING INTERFERENCE MICROSCOPES

Application of the full-duplication method

1. Measuring path differences by means of the flat-tints process (full-duplication process)

2. Measuring path differences, applying the fringes process (full-duplication process)

3. Determining the refraction index from path-difference measurement (full-duplication process)

4. Measuring the refraction index of liquids (full-duplication process)

\section{Application of the differential method}

5. Measuring path difference using the flat-tints method (differential process)

6. Fringe-shift path-difference measurements (differential process) 203

7. Determining the refraction index from path-difference measurements 205

8. Measuring the refraction index of liquids (differential process) 206

9. Measuring birefringence through a polarizing interference microscope 210

10. Conclusion

Chapter Vili. Measuring Thickness and Slopes with Interference Microscopes. Measuring Dry Masses

1. Transparent objects

2. Reflective objects (thickness measurement) 
3. Measuring angles of contact between liquid and solid surfaces

4. Measuring dry masses by means of an interference microscope

Chapter IX. Infra-Red AND Ultra-violet Microscopy

1. Objectives of infra-red and ultra-violet microscopes

2. Observing and recording infra-red and ultra-violet imagery

3. Fluorescence

\section{Chapter X. Microspectroscopy AND MicrospeCtrophotometry}

1. Fundamentals of microspectroscopy and microspectrophotometry

2. The object is illuminated in monochromatic light

3 . The object is illuminated in white light

4. Microspectrophotometric measurements

5. Causes of errors in microspectrophotometry

6. Photometric eyepieces

7. Fluorescence microspectrophotometry

\section{Chapter XI. Chemical Microscopy}

1. Introduction

2. Hot stage and cold stage

3. Calibrating the hot stage

4. Determining the melting point

5. Measuring the refraction index of a melted substance

6. Köfler's contact method

7. Temperature composition diagram of binary mixtures

8. Application of fusion methods

9. Characterizing and identifying fusible compounds 



\section{Foreword}

THE extensive development of microscopy, whether in the field of methods or instrumentation, requires from the user an ever-growing knowledge regarding the phenomena on which modern techniques are based. The images observed and the frequently tricky measurements made by means of complex instruments must be interpreted correctly. This is the reason why Chapter I is devoted to image formation in the microscope. The image of a pinpoint light-source is first examined, followed by an investigation of extended objects according to the type of illumination used. The mathematical developments related to such matters are not gone into and the object of this work will be only to emphasize general principles and basic results.

This preliminary survey serves as introduction to the chapters that follow in which observation methods in visible, ultra-violet and infra-red light are set forth, together with geometrical and physical measurements and fundamentals of microspectrophotometry and chemical microscopy.

Measurements of path differences in interference microscopy and their application to the determination of refractive indices and thicknesses are fully gone into. Lastly, the description of new devices will be generally preceded by that of more conventional instruments in order to make quite clear the underlying principles of up-to-date techniques and instrumentation.

Paris

M. FrançON 



\section{CHAPTER I}

\section{Physical Aspects of Image Formation in Microscopy}

\section{INTRODUCTION}

MicrosCOPIC investigation of image formation and interpretation cannot be based solely on considerations of geometrical optics. In order to ascertain the distribution of light-intensities in the images exhibited by a microscope, the laws of physical optics have to be brought into play.

The first and simplest case to consider is that of an object sufficiently small as to be likened to a pin-point of light. In the image, the light is distributed according to a law determined by diffraction. This image is the elementary diffraction pattern known as Airy's disk: it is the perfect image that would be exhibited by an aberration-free and perfectly focused microscope.

Images of large objects are all derived from the more or less complex process of such an elementary picture since any object may be split into elements small enough to be considered as discrete pin-point sources. Therefore, under such conditions, it can be anticipated that diffraction effects shall be the more prominent as the dimensions of the object are closer to those of Airy's disk. The finer details of a specimen are those which are the more markedly altered by diffraction. Since, naturally, it is such details that are the most valuable to microscopists, it is essential to determine the results given by the diffraction theory in order to reduce interpretation errors to a bare minimum.

However, in microscopy, the problem is rather the converse: it is not a given image which needs ascertaining: it is the actual structure of the object corresponding to it. No difficulty is involved when the size of the object is large in relation to the diffraction pattern as the geometrical aspect of the image prevails. Such is not the case when the size of the object approximates that of Airy's disk. Then a com- 
parison is to be proceeded with, by reverting to the problem as considered under its initial aspect. Since the structure of images of typical objects such as disks, lines, rectangles, edges and so forth, is known beforehand, the geometry of the detail examined can be determined by comparison. This applies not only to specimens whose details are featured by differences in brightness but also to thoroughly transparent specimens in which details consist solely in refraction-index and thickness differences. Therefore, more often than not, it is by close examination and comparison of the images, together with the theoretical results obtained, that the problem of image interpretation may be solved.

\section{IMAGE OF A LUMINOUS POINT EXHIBITED BY THE OBJECTIVE OF A MICROSCOPE IN MONOCHROMATIC LIGHT}

Figure 1.1 shows a microscope diagrammatically, $O_{1}$ being the objective and $O_{2}$ the eyepiece. $O_{1}$ is, for instance, the first front lens of the objective. The specimen $P$ emits light only through a small
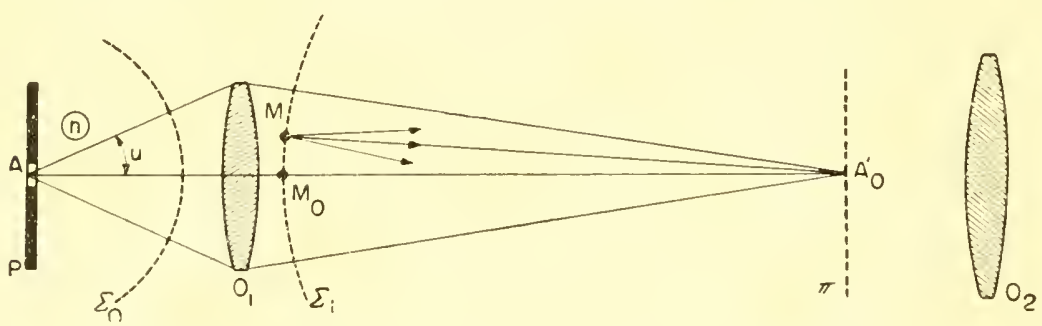

FlG. 1.1. Image of a luminous point produced by a microscope objective.

aperture whose size is far smaller than the resolving power of the objective and plays the part of the object. Assuming the light emitted by $A$ to be monochromatic, $n$ is the refraction index of the objectmedium and $u$ the angle under which the light-ray of the first objective lens is seen from $A$.

The objective $O_{1}$ (numerical aperture (N. A.) $n \sin u$ ) shows in $A_{0}^{\prime}$ an image of $A$. Required: to know the structure of the image $A_{0}^{\prime}$, i.e. the distribution of light-intensity in the plane $\pi$, across $A_{0}^{\prime}$.

The sphere $\Sigma_{0}$ (centre at $A$ ), is a wave surface in the object space. Assuming the objective $O_{1}$ to be perfect, wave surfaces in image space are spheres such as $\Sigma_{i}$. The centres of these waves are at point $A_{0}^{\prime}$. which is a geometrical image of $A$. According to Huyghens' principle. the image $A_{0}^{\prime}$ is formed as if the various points of the wave surface $I_{i}$ were actual sources of light in the same vibratory state. Any point, 
such as $M$, emits luminous vibrations, not only towards the geometrical light-ray $M A_{0}^{\prime}$ but in other directions as well. The various points of the wave surface $\Sigma_{i}$ diffract the light which overspreads on the image surrounding the point $A_{0}^{\prime}$.

Figure 1.2 shows that, $A_{0}^{\prime}$ being the centre of the sphere $\Sigma_{i}$, all the vibrations, originated from this wave surface, reach $A_{0}^{\prime}$ in the same vibratory state. In order not to over-elaborate the figure, only the vibrations emitted by two points, $M_{0}$ and $M_{1}$ are shown. The luminous amplitude, at $A_{0}^{\prime}$ is merely the sum of amplitudes of all vibrations reaching such point. Since intensity of light is proportional to the squared amplitude, squaring of the aforesaid sum determines intensity at $A_{0}^{\prime}$. Hence, light is maximum at $A_{0}^{\prime}$.

Let us now consider the diffracted vibrations reaching the random point $A_{1}^{\prime}$ of the plane $\pi$ (Fig. 1.3). Since point $A_{1}^{\prime}$ is removed from the sphere's centre, the distances from the various points of $\Sigma_{i}$ to $A_{1}^{\prime}$ are no longer equal. Hence, the vibrations emitted by $\Sigma_{i}$ do not reach $A_{1}^{\prime}$ in the same condition. In the case of Fig. 1.3, both points $M_{0}$ and $M$ emit vibrations which are in opposition at $A_{1}^{\prime}$. If $\lambda_{\text {. is the }}$ wave-length, then: $A_{1}^{\prime} M-A_{1}^{\prime} M_{0}=\lambda / 2$ : both vibrations are cancelled out: the luminous amplitude at $A_{1}^{\prime}$, originated by $M_{0}$ and $M$ is zero.

It follows that intensity at $A_{1}^{\prime}$ is the outcome of all points of the wave surface $\Sigma_{i}$ and not merely that of points $M_{0}$ and $M$ only.

Computing the phenomenon discloses the following: the image of the luminous point $A$ is shown by a very bright, circular, central disk (Fig. 1.4), surrounded by alternately bright and black rings whose intensity decreases rapidly as distance increases. Only two or three luminous rings are usually visible in a microscope as the others are absorbed by the stray light and, therefore, too weak. The curve in Fig. 1.5 shows the distribution of light-intensity in the diffraction pattern. Ordinates show the intensities; abscissae are determined by the parameter $Z$ which depends not only on the geometrical distance from the centre $A_{0}^{\prime}$ but also on the characteristics of the microscope's objective. If $g$ is the objective magnification and $C$ the geometrical distance from the point $A_{0}^{\prime}$ to the point where the light intensity is to be calculated, the diffraction theory conduces to write:

$$
Z=\frac{2 \pi n \sin u}{\lambda} \frac{C}{g}
$$



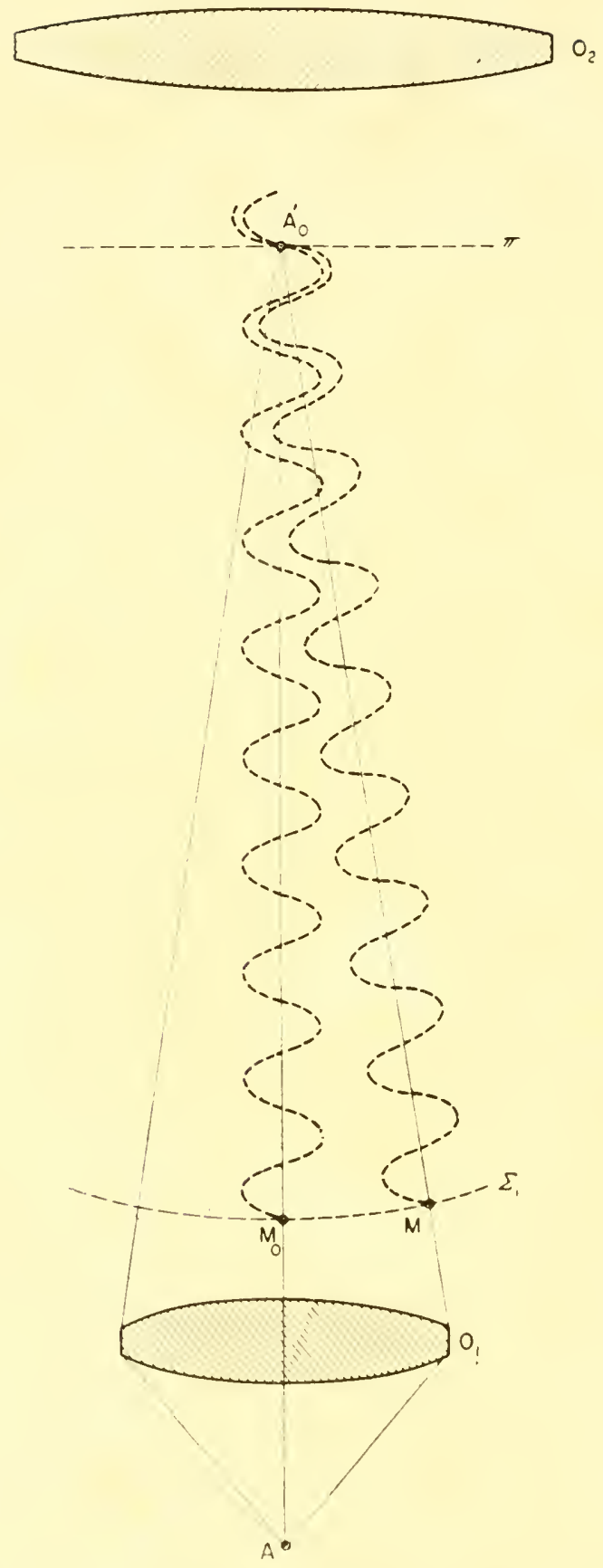

FIG. 1.2. $M_{0}$ and $M$ emit vibrations which are in phase at $A_{0}^{\prime}$. 
PHYSICAL ASPECTS OF IMAGE FORMATION

5
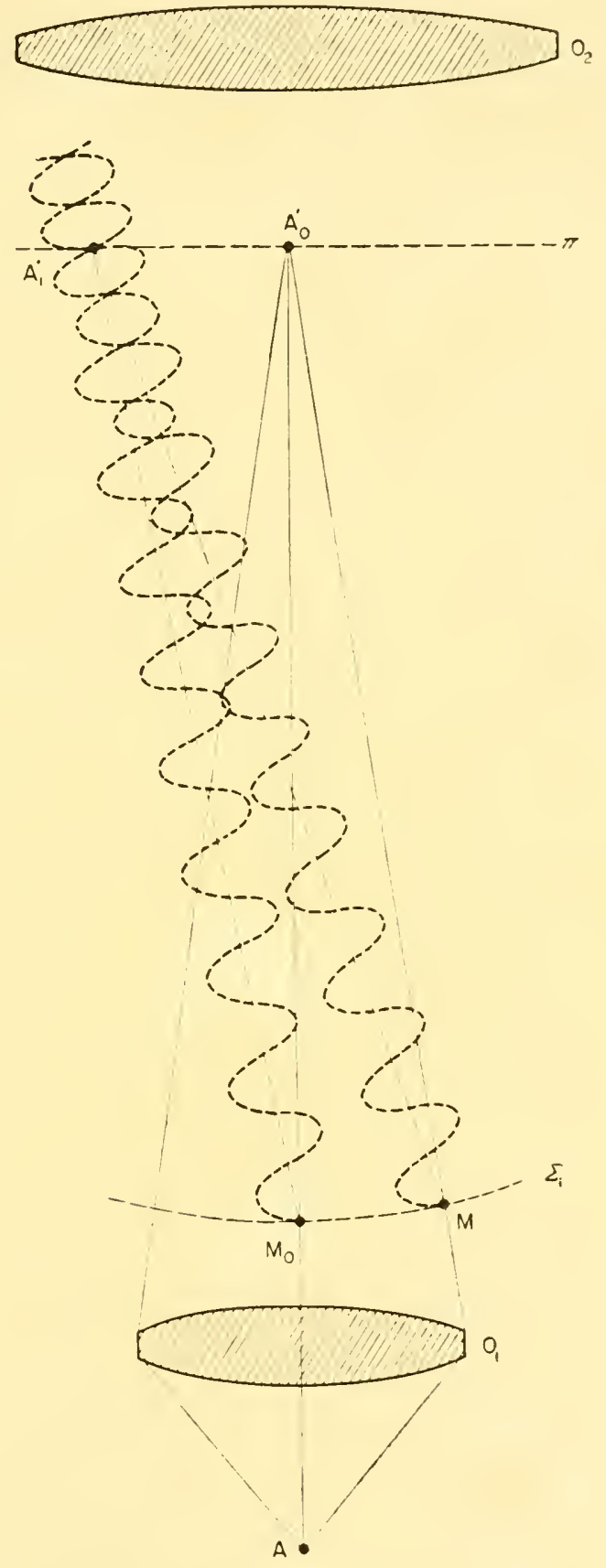

FIG. 1.3. The vibrations are in opposition at $A_{1}^{\prime}$. 


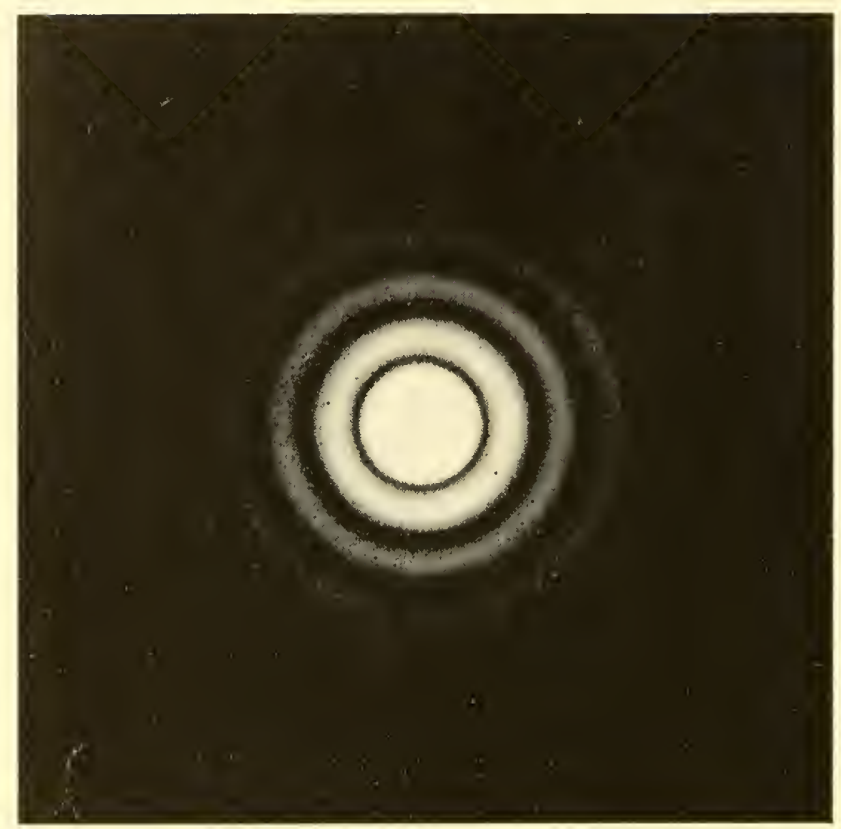

FIG. 1.4. Airy disk.

Positions of the black rings are shown to correspond to $Z=3.83$ $7.02 \quad 10 \cdot 17 \ldots$ and positions of peaks to $Z=0 \quad 5.14 \quad 8.46 \quad 11.62 \ldots$ $\begin{array}{lllll}\text { Peak intensities are } 1 & 0.0175 & 0.0042 & 0.0016 .\end{array}$

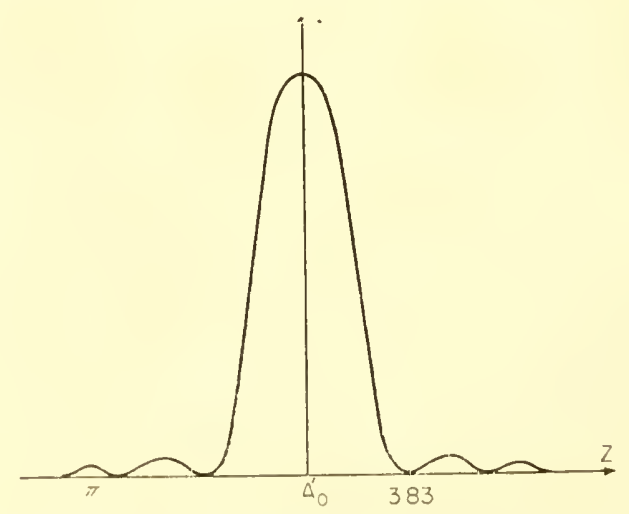

FIG. 1.5. Light distribution in the Airy disk.

The first dark ring corresponds to $Z=3.83$; therefore, the linear radius $C_{0}$ of the central diffaction in the image plane $\pi$ can be cal- 
culated. Substituting $Z$ for 3.83 gives,

$$
C_{0}=\frac{1 \cdot 22 \lambda}{2 n \sin u} g \text {. }
$$

As a matter of fact, it is the central disk which acts as the imaged point $A$. The luminous rings surrounding the central disk merely enlarge it and are spurious light.

It follows that enlarging the image is always detrimental and, to observe very fine details, the diameter of the central spot is to be as minute as possible. This result is achieved, in accordance with equation 1.2, by using a high numerical aperture objective. Let us take for example, a N. A. $n \sin u=0.30$ with magnification $g=25$. At wave-length $\lambda=0.6$ microns, $C_{0}=30$ microns. Phenomena can be considered in the specimen plane, whereupon they occur as if the pinpoint object $A$ were substituted for a small disk having $C_{0} g=1.22 \lambda /(2 n \sin u)$ as radius. With a $1.30 \mathrm{~N}$. A. objective the diameter of the central diffraction disk drops to 0.28 microns for the same wave-length. In the specimen plane, this diameter depends solely on the objective N.A. and the wave-length of the light used.

Figure 1.6 shows the diffraction patterns of various numerical apertures for the same intensity in the disk centres.
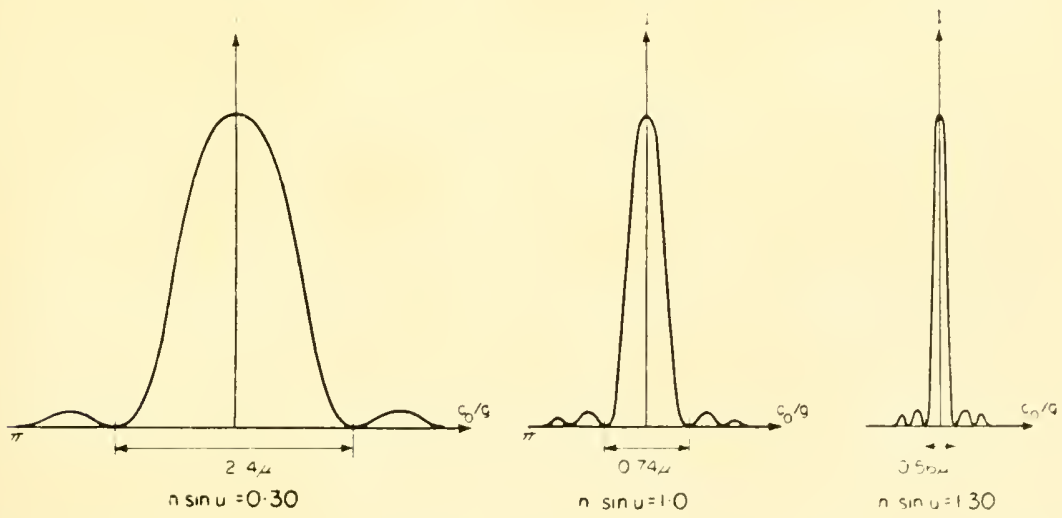

FIc. 1.6. Diffraction patterns with various numerical apertures in the specimen plane (same intensity in the disk centres) $(\lambda=0.6 \mu)$.

However, it should be mentioned that the foregoing applies strictly to low or medium numerical apertures only. When high numerical apertures are involved, A. Wolf has shown that the disk is somewhat 
enlarged and that minimums are no longer zero. Enlargement is in the region of 8 per cent for an aperture $\sin u=0.85$. Alterations of the diffraction pattern are therefore small and may be neglected in a first approximation.

\section{IMAGE OF A LUMINOUS POINT ORIGINATED BY A REFLECTING OBJECTIVE}

It may occur occasionally that a portion of the $\Sigma_{i}$ wave, originated by the objective, is masked by an opaque screen as, e.g. in a reflecting objective. Many types of reflecting objective are used in microscopy, but from the standpoint now under discussion, they may all be likened to the diagram in Fig. 1.7.

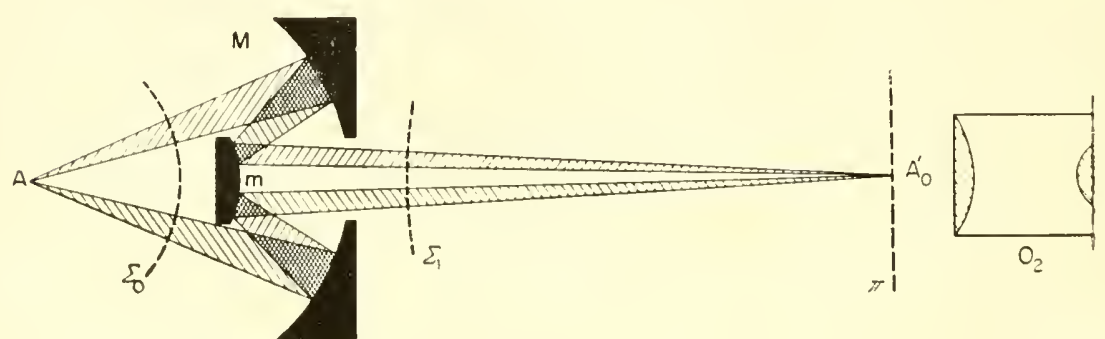

FIG. 1.7. Diagram of the ray paths through a reflecting objective.

In this type of objective. the light. originated by the object $A$, is reflected first by the concave mirror $M$ then by the convex mirror $m$ and ends at the image $A_{0}^{\prime}$ being examined through the eyepiece $O_{2}$. The small mirror $m$ screens the beam which is reflected on $M$.

The surface waves $\Sigma_{0}$ and $\Sigma_{i}$ are ring-shaped and the diffraction pattern is altered. Curve (1) in Fig. 1.8 shows the distribution of intensity in the conventional diffraction disk brought about by the whole wave (Airy's disk). Curve (2) shows the structure of the diffraction disk when the wave is masked in the central area by a circular opaque screen. If the small mirror is kept in position by three thin strips $120^{\circ}$ apart, another perturbation of the diffraction phenomenon is developed: light is accumulated in three $120^{\circ}$ directions.

Curve (2) in Fig. 1.8 shows that screening the beam reduces the central disk, which would be beneficial were it not that the luminous rings are intensified. When extended high-contrast objects are dealt with. there is nevertheless a gain, but the intensified rings originate 
spurious diffraction fringes making observation uncomfortable. Obturation also greatly reduces the definition of extended objects of low contrast. this conducing to reduce the diameter of mirror $m$ as much

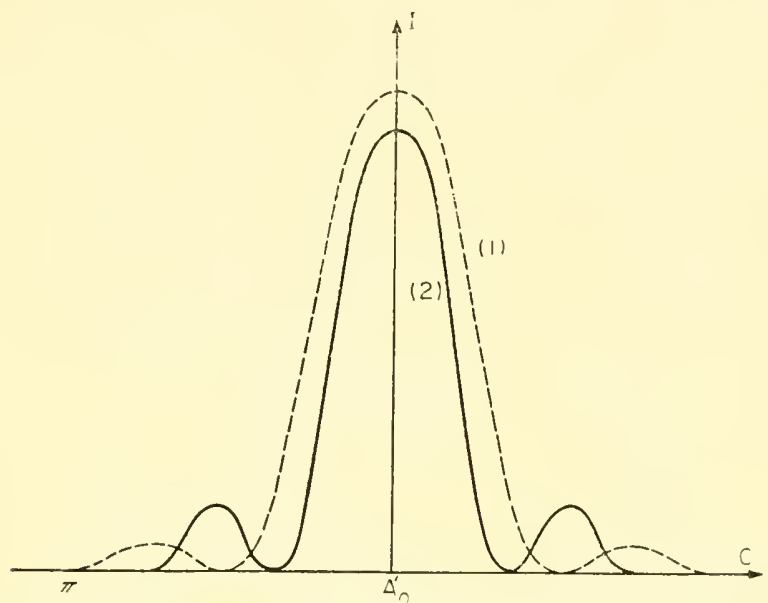

FIG. 1.8. (1) Airy disk: (2) Diffraction pattern with a reflecting objective.

as possible. Provided the masked area does not exceed 10 per cent of the whole area of the wave $\Sigma_{i}$, it can be assumed that the diffraction phenomenon is not substantially altered.

\section{IMAGE OF A LUMINOUS POINT WHEN THE MICROSCOPE IS NOT PROPERLY FOCUSED (MONOCHROMATIC LIGHT)}

The foregoing images $(\S 2)$ require an excellent objective and very precise focusing. Now, microscopists frequently alter focusing during observations in order to ascertain that any particular detail is seen under the best conditions. Therefore, it is desirable to go into the diffraction-pattern changes taking place when focusing is not carried out in the plane of the perfect image but slightly in front of or behind it.

Let us consider Fig. 1.9 which is a simplified version of Fig. 1.2. As the microscope is no longer faultlessly focused, phenomena occur as if the eyepiece were focused on the plane $\pi$ at the short distance $x$ from $\pi$. The luminous vibrations, diffracted by the various points of the surface wave $\Sigma_{i}$, do not reach the point $B^{\prime}$ at the time because said point is not the centre of the sphere $\Sigma_{i}$. Point $B^{\prime}$ is the centre of sphere $\Sigma_{i}^{\prime}$ which is removed from sphere $\Sigma_{i}$ by a distance $A$ that increases as the wave edge is neared. The vibrations, originated by $M$, 
travel farther than do those originated by $M_{0}$. The difference between

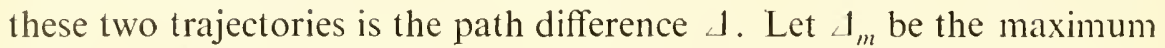
value of $\Delta$ which correspond to the edge of the wave. The depth of focus may be characterised by the path difference $J_{m}$. If $\iota_{m}=0$, focusing is carried out on the plane of the flawless image whose structure was investigated previously. For a $\perp_{m}$ value other than zero, the focusing
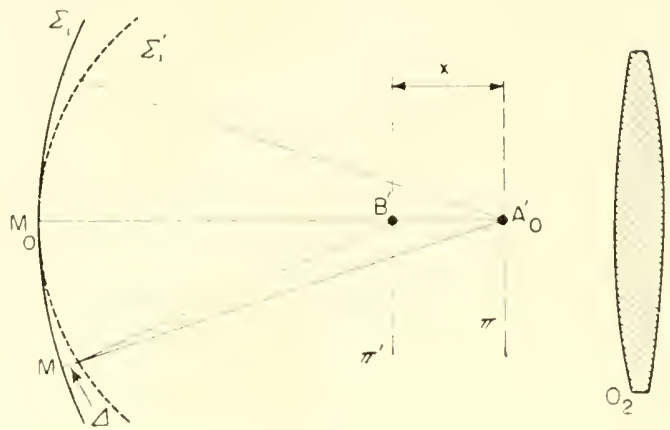

FIG. 1.9. Path difference $\angle$ produced by a shift of the focusing plane.

plane no longer passes through the flawless image $A_{0}^{\prime}$. Assuming the focusing to be carried out at $B^{\prime}$, at the short distance $x$ from $A_{0}^{\prime}$, what would be the structure of the image observed? It follows that the diffraction pattern at $B^{\prime}$ depends on the defective focusing, i.e. on $I_{m}$. However, in general, decreased intensity of the central peak and intensified luminous rings are observed.

Figure 1.10 shows this. Curve (1) illustrates Airy's disk and curve (2) the diffraction disk structure when focusing is but slightly defective. The diffraction disk is blurred and the image contrasts of extended objects greatly impaired. When out-of-focus becomes marked. the diffraction disk structure is completely altered. Such effects are readily observed in the microscope by using as specimen an object-slide, processed by a vacuum-evaporated aluminium-metallisation and covered by a cover-slip if the objective is of the dry-front lens type. The aluminium coating of such object-slide is never completely opaque: there always remain small holes forming ideal pin-point sources of light.

Figure 1.11 shows how the first luminous rings are accentuated when defective focusing has a $L_{m}$ value smaller than $\lambda$. When $I_{m}=\lambda$. Fig. 1.12 shows the minimum to be zero in the centre of the diffraction disk. Light-intensity changes can be evinced by a curve 
showing changes in intensity when focusing is altered in the centre of the diffraction disk. Results are shown in Fig. 1.13. Focusing changes are made by fine focusing movements but Fig. 1.13 can be

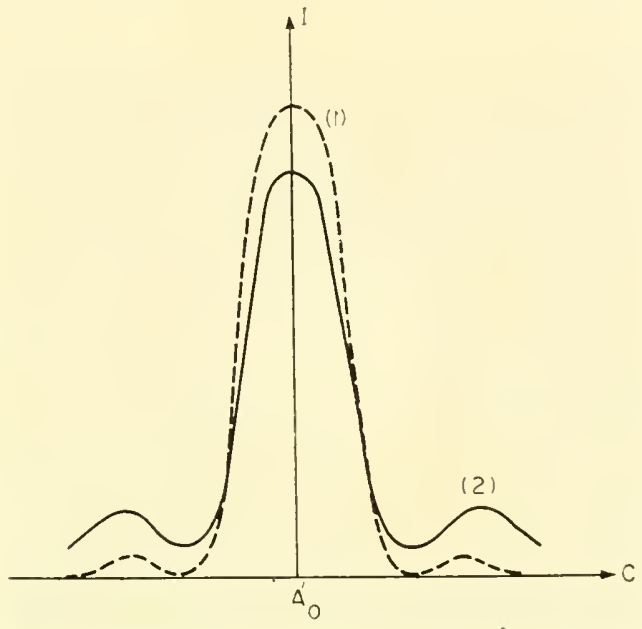

FIG. 1.10. (1) Airy disk; (2) Diffraction pattern when focusing is but slightly defective.

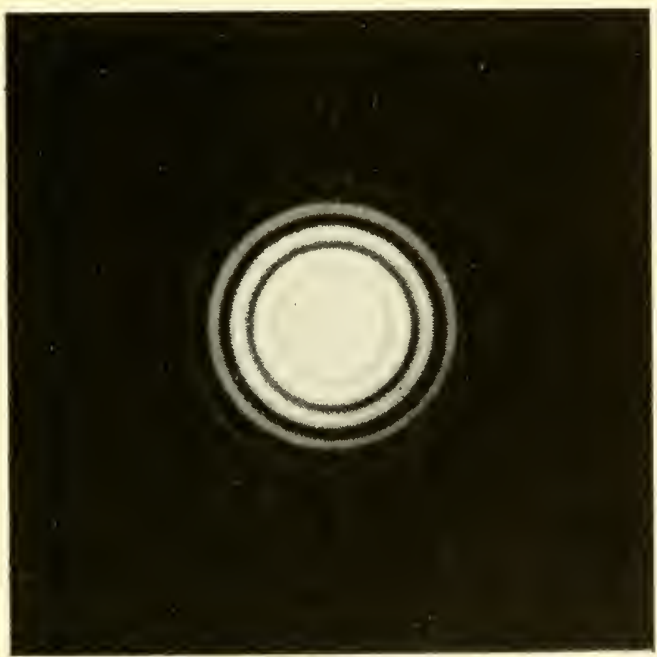

FIG. 1.11. Diffraction pattern when defective focusing has a value smaller than $\lambda$.

interpreted more readily assuming that it was the eyepiece that moved. If the eyepiece is focused at $A_{0}^{\prime}$ peak intensity $I_{0}$ is in the centre of the diffraction pattern. The curve (Fig. 1.13) shows that defective focusing 
decreases intensity in the centre. If the eyepiece is properly focused on a plane lying at a distance from the perfect image equal to $B^{\prime} A_{0}^{\prime}$, the disk-image appears as shown in Fig. 1.12. Figure 1.13 evinces that diffraction patterns exhibiting dark centres in front of and at

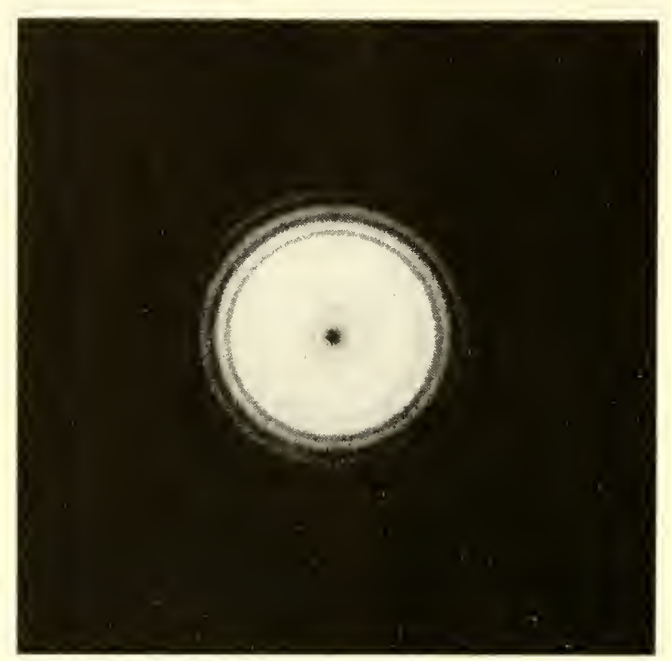

FIG. 1.12. Diffraction pattern when $d=\%$

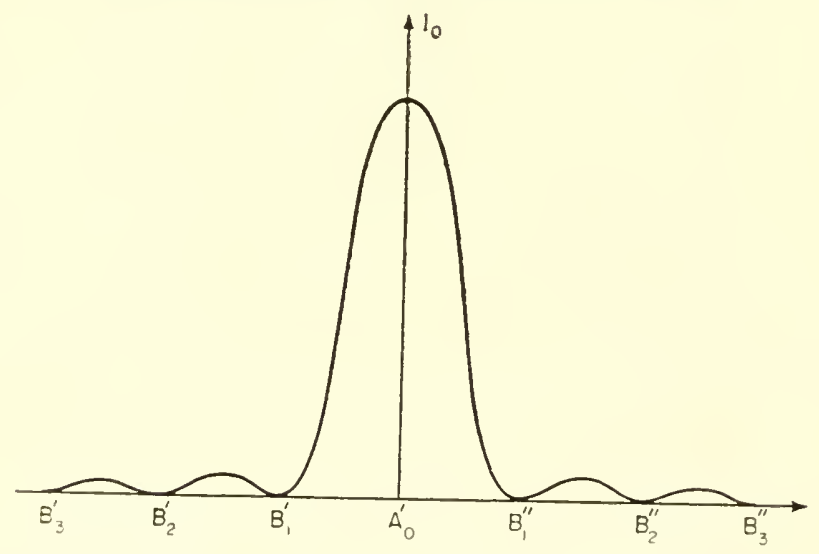

FIG. 1.13. Light-intensity changes in the centre of the diffraclion pattern when focusing is altered.

the back of Airy's disk are again recurrently met with. However, the intensity peak values in the disk centres become very low. These phenomena can be conveniently observed by means of a high magni- 
fication substantially diaphragmed-down objective in conjunction with a powerful eyepiece.

Figure 1.14 shows the results achieved. The isophotal lines, close to $A_{0}^{\prime}$, are plotted on the plane $M_{0} A_{0}^{\prime} M$ (Figs. 1.1, 1.2, 1.3) but, as the phenomena are of revolution about the centre-line $A A_{0}^{\prime}$ of the microscope, results are identical on any plane provided the latter passes through $A A_{0}^{\prime}$. In order to visualize the phenomena occurring in space, Fig. 1.14 should be rotated about the axis $A M_{0} A_{0}^{\prime}$. Cutting the resulting volume by a plane at right angles to $A A_{0}^{\prime}$, passing through $A_{0}^{\prime}$ (shown as $\pi$ in Fig. 1.14), will evince Airy's disk. The straight lines $M^{\prime} A_{0}^{\prime}$ and $M A_{0}^{\prime}$ are light-rays limiting the geometrical beam. The dark dots, plotted on the figure, show the positions of zero minimums of Airy's disk (dark rings). Correlation with Fig. 1.5 can be established since intersection of isophotal lines with $A A_{0}^{\prime}$ show the intensities obtaining along the microscope centre-line. The dark dots, plotted along $A A_{0}^{\prime}$, show the position of dark-centre diffraction patterns and correspond to points $B_{1}^{\prime}, B_{2}^{\prime} \ldots B_{1}^{\prime \prime}, B_{2}^{\prime \prime} \ldots$, in Fig. 1.13.

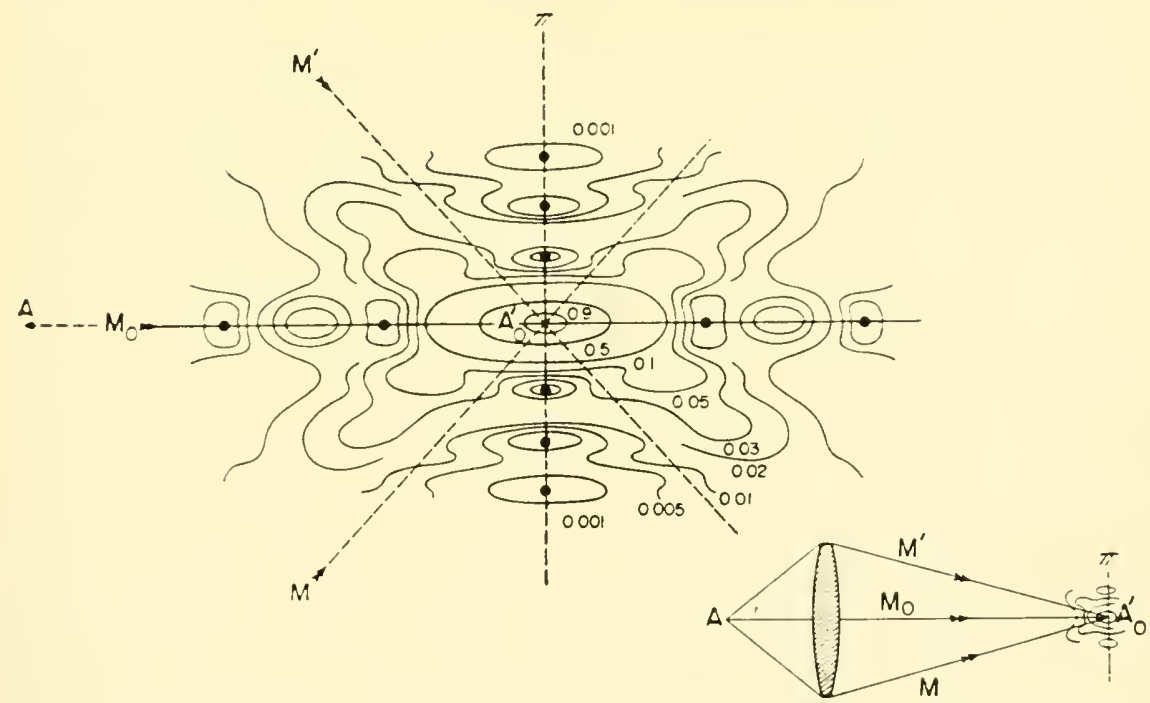

FIG. 1.14. Isophotes in a meridional plane (After E. H. Linfoot and E. Wolf).

Figure 1.14 shows at once that, on either side of the plane $\pi$, diffraction-patterns are identical, unless aberrations of the microscope objective are inadequately corrected. This is a simple and accurate way of evaluating the quality of an objective. Let us take the above- 
mentioned aluminium-based coating and a high-magnification eyepiece. After selecting a hole small enough to exhibit the conventional features of Airy's disk, it is brought in the centre of the field and the fine adjustment actuated. Then, the microscope is brought nearer the specimen whereupon the structure of the diffraction-disk is materially changed. Now, reverting to proper focusing, the operation is resumed by moving the objective away from the specimen. If the objective is well corrected, the same successive aspects as before are retained. If, conversely, the objective is not well corrected the aspect of the diffraction patterns is very different. On one side many diffraction rings are seen whereas, on the other, there are more or less uniform blobs. Strange as it may seem, such examination is quite easy to carry out, even by a layman, because the eye is very sensitive to small differences in appearance provided that the to-and-fro motion imparted to the focusing device be not too slow.

\section{IMAGE OF A LUMINOUS POINT IN WHITE LIGHT}

Owing to chromatism of the objective, images correlated to the various wave-lengths of the spectrum are not focused simultaneously. Assuming that the image $A_{0}^{\prime}$, correlated to wave-length $i_{\nu}=0.56 \mu$ in Fig. 1.15, is being focused: at wave-length $\lambda=0.65 \mu$, for instance,

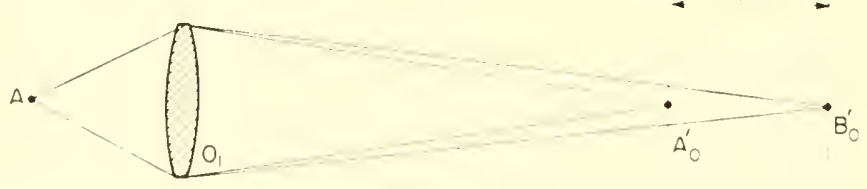

FIG. 1.15. Depth of fozus $x$ produced by chromatism.

the image would be at $B_{0}^{\prime}$ and the depth of focus equal to $x$. Image structures originated by the various radiations can be theoretically computed. Intensity at any point of the focusing plane $\pi$ equates the sum, at the said point, of the total illumination generated by the various radiations. It follows that the spectral distribution of energy emitted by the source and the speetral responsiveness of the receiver, (either the eye or photographic plate) are to be taken into account.

In perfectly chromatically-corrected microscope objectives as, e.g. in reflecting objectives, all images correlated to the various wave-lengths are Airy's disks in the plane 7 . Formula 1.2 shows that red images 
are larger than blue ones as diffraction gives rise to slight chromatism. This effect is hardly perceived in the central disk. Conversely, it may be detected in the first ring which is white in the central area but slightly iridescent along the edges. Such iridescence is red along the outer edge and blue along the inner.

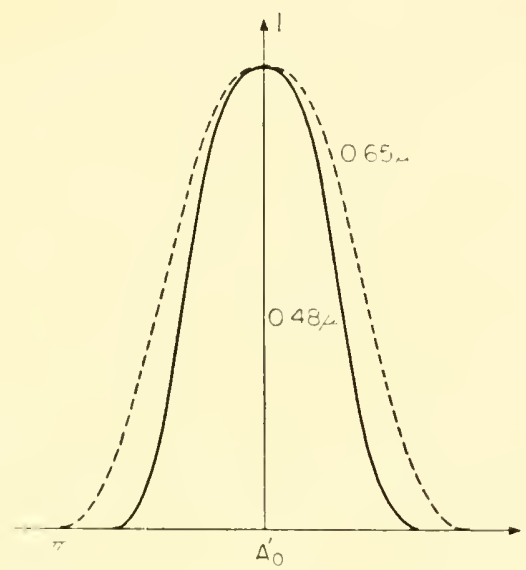

FIG. 1.16. Chromatism produced by diffraction.

Two Airy's disks are shown in Fig. 1.16: they correspond to the radiations $C(0.56 \mu)$ and $F(0.486 \mu)$, respectively. Abscissae show the relative widths of the two central disks, on the basis of identical intensity in the disk centres. This figure only shows the disk enlargement due to diffraction. As mentioned above, obtaining the actual ordinates requires taking into account the spectral characteristics of both source and receiver.

Figure 1.17 shows actual aspects when the source has an equivalentenergy spectrum and the receiver is an average eye whose spectral sensitiveness is given by the Gibson and Tyndall curve (Fig. 1.18). The $C$ and $F$ radiations are close to the ends of this curve and, therefore, but slightly efficient from the visual standpoint. Comparatively, the diffraction disk, corresponding to peak eye sensitiveness $(0.555 \mu)$, would have an intensity equal to 18.7 at the centre (Fig. 1.17).

These results do not apply to lens-type objectives the chromatism of which is never perfectly corrected. In order to compare the chromatism of microscope objectives, the chromatism curves, viz. the focal points (perfect images), are shown versus the wave-length. Such curves are not adequate to define image quality as the latter depends not 
only on longitudinal chromatism (distance between focal points) but also on the numerical aperture $n \sin u$ and the magnifying power $g$ of the objective. We think it advisable to show direct the diffraction-

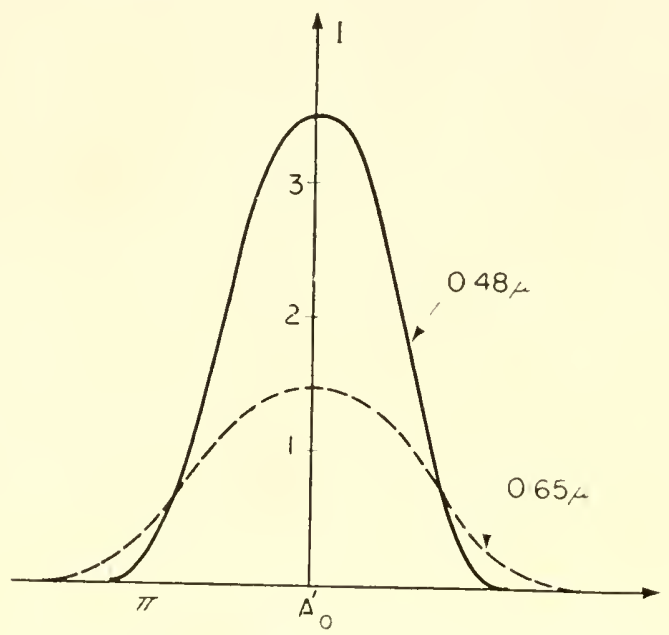

FIG. 1.17. Actual aspects of the diffraction pattern.

pattern structure in white light for three types of objectives: achromatic, fluorite and apochromatic. Computation was carried out taking the

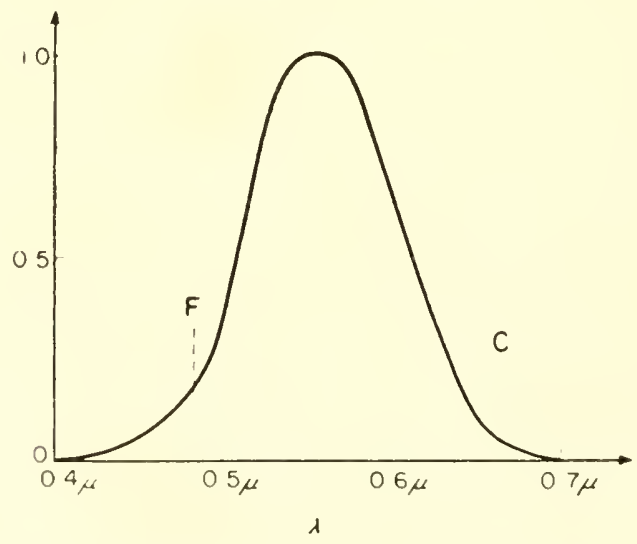

FIG. 1.18. Gibson and Tyndall curve of the spectral sensitivity of the eye.

eye as receiver (Gibson and Tyndall curve) and a standard microscope lamp as source whose spectral distribution of energy can be likened to the photometrists' standard A (Fig. 1.19). 
Results are shown by the curves in Fig. 1.20. Abscissae evince. in the focusing plane, the distance (in microns) to the geometrical centre of the image while ordinates show the luminous intensities, taking as intensity unit that obtaining in the centre of the diffraction pattern produced by the apochromatic objective. In the three objectives, then, $n \sin u=0.95$ and $g=60$.

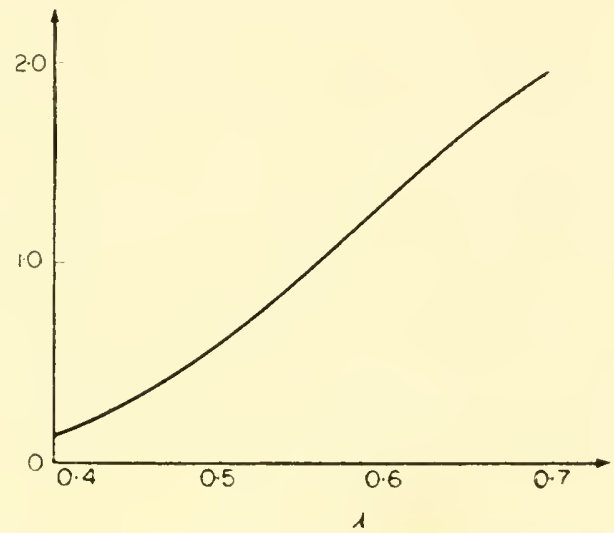

FIG. 1.19. Spectral distribution of energy of the photometrists standard $A$.

The maximum spectral distances from $\mathrm{C}$ to $\mathrm{F}$ (longitudinal chromatism), selected for calculations, are:

apochromatic objective $x=1 \mathrm{~mm}$

fluorite objective $\quad x=4 \mathrm{~mm}$

achromatic objective $\quad x=8 \mathrm{~mm}$.

Curve (a) shows the image structure afforded by the apochromatic objective. Ordinates, in this and the following curve, were multiplied by 40 from the abscissa 20 onwards. There are no zero minimums any longer. Were the image, shown in this figure, produced by an objective completely devoid of chromatism, e.g. a reflecting objective, the differences would be minute. Central peak intensity would be 1.015 , while that of the first luminous ring would drop by 0.001 . Hence, the difference between an apochromatic and a perfect objective is very slight. In both cases, vanishing of the dark rings is due to a change in diameter of the diffraction disks taking place in accordance with the wave-length. Therefore, effects of the residual chromatism of the apochromatic objective are less prominent than those of diffraction-originated chromatism. 
Curve (b) corresponds to a fluorite objective: the first minimum. close to $20 \mu$ is more prominent: decreased image contrast may, therefore, be expected. In all these curves, the central disk, acting in fact as image, has a diameter of 20 microns approximately. Anything outside it is detrimental diffracted light. In an achromatic objective, the detrimental diffracted light is particularly marked and a drop of the central peak is evinced. Perception limit of small details is consequently much less satisfactory in an achromatic objective. Numerical values are shown later. On the other hand, the all-important colour of the diffraction pattern is not evinced in the curves shown in Fig. 1.20. The central disk, in both a perfect or an apochromatic objective, is definitely white. Only the first luminous ring exhibits a slight iridescence. Iridescence is more prominent in the fluorite objective and, in the achromatic objective the slightly coloured central disk is ringed by diffracted light whose colour is vivid (usually greent purple). As in achromatic objectives, the amount of diffracted ligh-

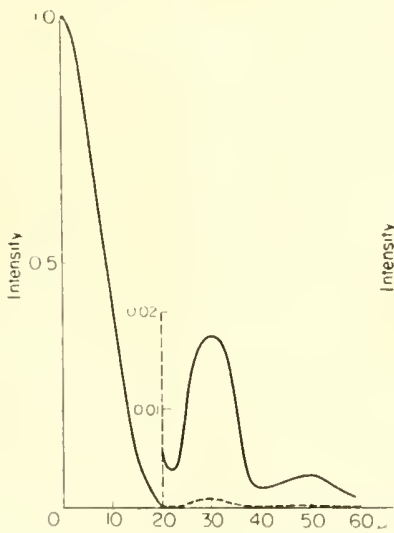

(a) Apachrort.ulic ablecilve

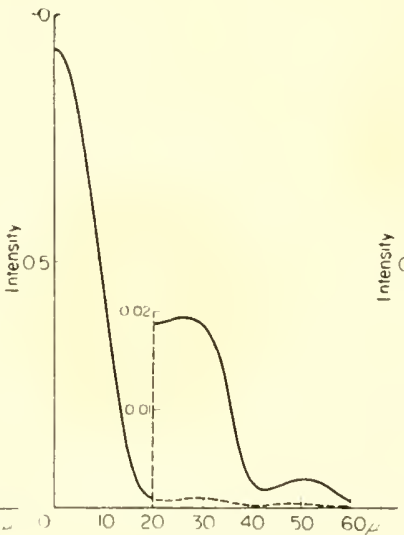

(b) Fluorite objective

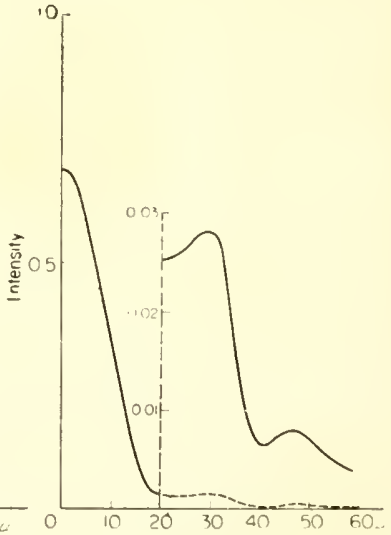

(c) Achromatic objecive

FIs, 1.20. Diffraction patterns: (a) - apochromatic objective; (b) - fluorite objective; (c) - achromatic objective.

outside the central disk is important and its detrimental effects on a coloured object can be readily visualized. Achromatic objectives should not be used, therefore, in colour photomicrography.

The foregoing performance shows the difference in quality between apochromatic and other lens-type objectives. The first bright ring. shown by an apochromatic objective, scarcely exceeds the intensity 
exhibited by a perfect objective. The first minimum is five times lower than that of a fluorite objective and seven times lower than that of an achromatic one.

Naturally, the structure of diffraction patterns may vary slightly with the chromatic correction of a given objective. Accordingly, the curves (Fig. 1.20) should merely be considered as showing the general aspect of the phenomena involved.

\section{LUMINOUS POINT IMAGED IN THE PRESENCE OF ABERRATIONS}

All the foregoing results apply to a perfectly corrected aberrationfree objective, the chromatism merely bringing about defective focusing but no wave-front deformation. When the objective is not perfectly aberration-corrected, the wave surface is no longer spherical thus entailing an altered diffraction disk. A portion of the luminous energy of the central diffraction disk is transferred to the rings which spread the image and lessen contrasts throughout. In Fig. 1.21, the sphere

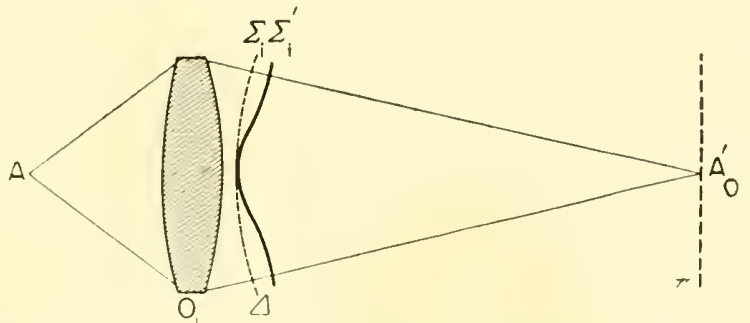

FIG. 1.21. Wave surface in presence of aberrations: $\Sigma_{i}^{\prime}$ is deformed.

$\Sigma_{i}$ represents the ideal surface-wave that would be achieved were the objective $O_{1}$ free from aberrations. As a matter of fact, aberrations distort the surface wave which becomes $\Sigma_{i}^{\prime}$. The distance between $\Sigma_{i}$ and $\Sigma_{i}^{\prime}$ is denoted by $\Delta$ and is the path difference between the actual wave $\Sigma_{i}^{\prime}$ and the reference sphere $\Sigma_{i}^{\prime}$. The $\angle$ values and variations are determined by the aberrations of the microscope objective. Going into the alterations brought about by aberrations in the diffraction disk structure is very helpful. Such alterations need investigating for they take effect on image formation and, hence, in their interpretation.

\section{Spherical aberration}

Let us assume that, notwithstanding its deformations the actual wave $\Sigma_{i}^{\prime}$ is a wave of revolution about the axis $A A_{0}^{\prime}$. In this case, 
the path difference $J$ at any point of the wave is merely a function of the distance to the axis of said point. Spherical aberration is present in the microscope objective. Such aberration is usually pretty well corrected and maximum values of the $\Delta$ deformation always small. In microscopy, the geometrical aspect of the phenomenon, i.e. development of a caustic curve, never occurs. With regard to defective focusing, it may be said, in general, that spherical aberration decreases the central peak of the diffraction pattern and eliminates the black rings as in Fig. 1.10. Provided the surface of the actual wave $\Sigma_{i}^{\prime}$ be enclosed between two spheres one quarter wave-length apart, it is considered that the diffraction-pattern structure does not differ materially of the Airy disk. This is Rayleigh's law, which serves to calculate objectives. The diffraction theory enables one to calculate structure

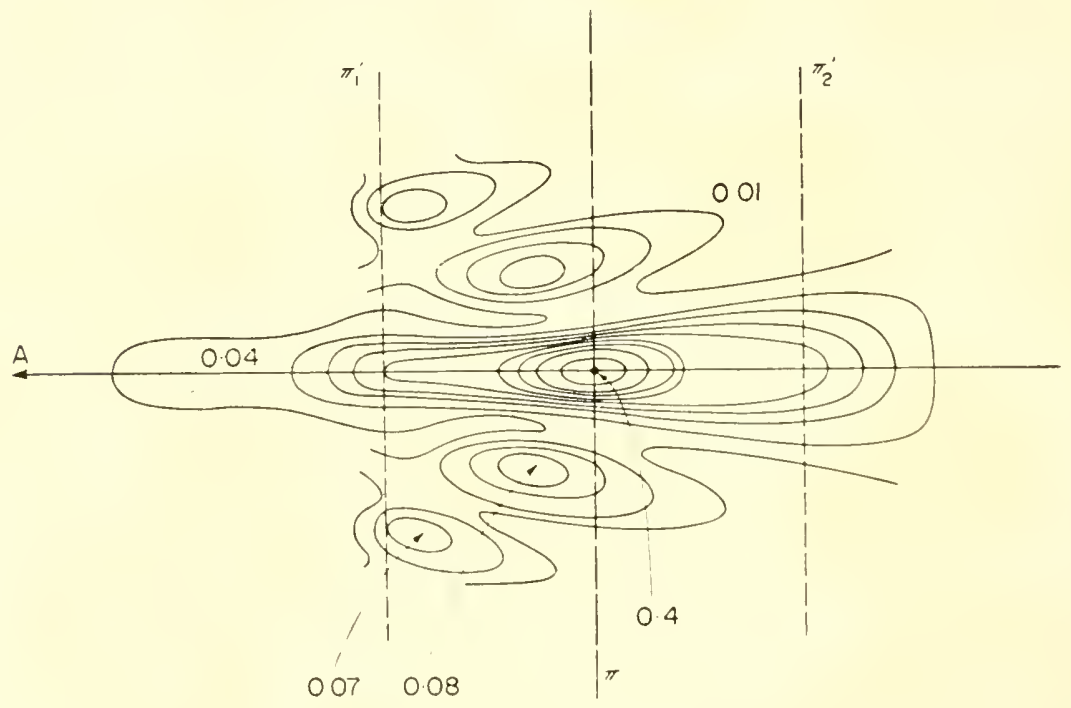

FIG. 1.22. Isophotes in a meridional plane in presence of spherical aberration (after J. Picht).

images in various planes parallel to the plane $\pi$ by combining spherical aberration and defective focusing. The phenomenon being one of revolution, the results can be shown by plotting the lines of equal intensity as in the case of Fig. 1.14. Figure 1.22 shows the result achieved when spherical aberration has a 1 peak value not exceeding $2 \lambda$. This time, obviously, the symmetry in relation to any plane perpendicular to $A A_{\theta}^{\prime}$ has vanished. If the best focusing plane is considered to be the plane $\pi$. 
the curves in Fig. 1.22 show that the diffraction pattern in the plane $\tau_{1}^{\prime}$ diverges from the diffraction pattern in the symmetrical plane $\tau_{.0}^{\prime}$. As mentioned in $\$ 4$, the differences in appearance observed on each side of the good image enable one to detect the objective's spherical aberration. When focusing on the side of the plane $\pi_{1}^{\prime}$, diffraction disks of varying complexity exhibit a fairly large number of diffraction rings. When focusing on the other side of $\pi$, a nearly uniform halo is visible, which grows larger as the focusing plane recedes from $\pi$. This method does not allow one to measure aberration quantitatively but merely to test it. However, it can be said that, if a small divergence in appearance is evinced to a trained eye, the objective's spherical aberration equates approximately the path difference $\lambda / 4$.

\section{Coma}

When the pin-point object and its image $A_{0}^{\prime}$ are along the axis (Fig. 1.1) and provided that the objective be corrected for both these points, the image $A_{0}^{\prime}$ is Airy's disk. The objective is stigmatic at points $A$ and $A_{0}^{\prime}$.

Let us now consider a luminous, pin-point object $B$, away from the axis at a short distance from $A$ (Fig. 1.23). If the stigmatism shown

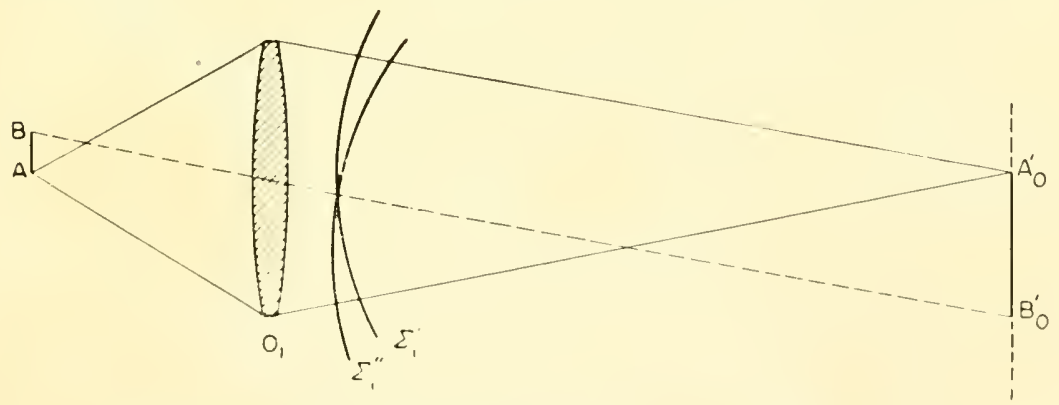

FIG. 1.23. Object $B$ and image $B_{0}^{\prime}$ out of the axis. Coma.

at points $A$ and $A_{0}^{\prime}$ does not hold good for both $B$ and its image $B_{0}^{\prime}$, the wave $\Sigma_{i}^{\prime \prime}$, related to points $B$ and $B_{0}^{\prime}$, is not spherical. The $B_{0}^{\prime}$ image is distorted and not of revolution; diffraction rings are no longer coaxial and the image has only one plane of symmetry. The image $B_{0}^{\prime}$ is shaped as a comet-tail and exhibits coma. Figure 1.24 shows the aspect of the diffraction pattern in presence of coma in a plane perpendicular to the microscope axis. The plotted lines show isophotal lines. In the case of the ideal diffraction pattern (Airy's disk) the 
isophotal lines shown in the same plane would be co-axial circumferences.

Provided the axial stigmatism requirement holds good at points $B$ and $B_{0}^{\prime}$, the objective meets Abbe's sine condition and is coma-cor-

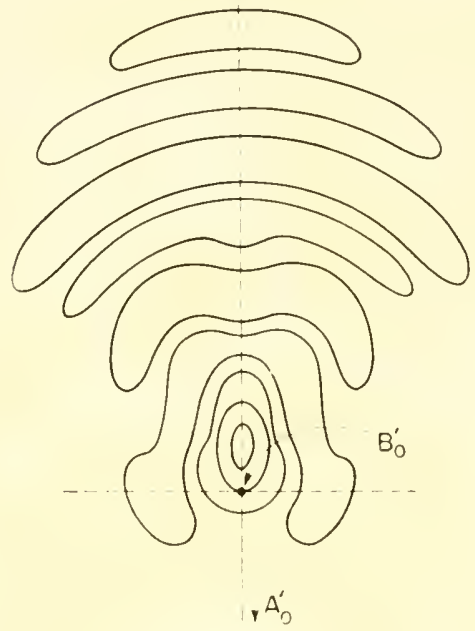

FIG. 1.24. Diffraction pattern in presence of coma. Isophotes after Nienhuis. If the system is very diaphragmed the image is located at $B_{0}^{\prime}$.

rected. This applies to microscope objectives. Such aberration is not readily observed in microscopy. With some objectives, it may be detected but may frequently be mistaken for astigmatism.

\section{Field curvature. Astigmatism}

The image $A_{0}^{\prime} B_{0}^{\prime}$ of a small flat object $A B$, as shown by a microscope objective, is not flat in general: the image is no longer located on the plane $\pi$ but on a surface of revolution the concave side of which is on the objective side. The objective exhibits some field curvature which is an aberration inherent in alt microscope objectives. From the standpoint of pattern-figure structure it acts as a focusing defect does. Field curvature is not objectionable in microscopy in the case of visual observation which is frequently restricted to a small detail in the field centre. Conversely, the use of plane-field objectives, the planachomats, is very effective in pholomicrography.

When observing the image $B_{0}^{\prime}$ of the luminous point $B$ when the former is close to the edge of the field of view, no focusing adjustment 
will provide the conventional diffraction disk (Airy"s disk). Imparting a to-and-fro motion to the focusing button discloses a small streak of light, pointing to the field centre, and another one, at right angles

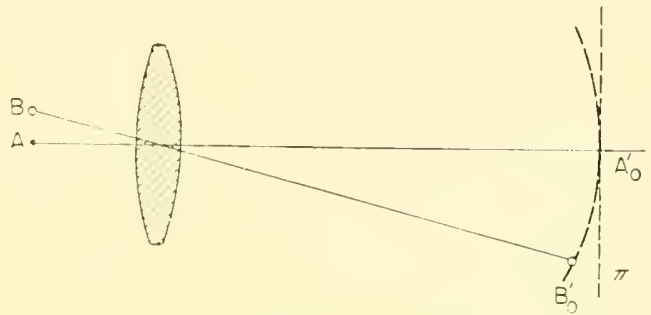

FIG. 1.25. Field curvature.

to the former: the objective is exhibiting astigmatism. These two small right-hand segments, $90^{\circ}$ from one another and lying in two different focusing planes, are astigmatic focal lines: they are mainly seen at the field edges. Figure 1.26 shows such a focal line. The diffraction fringes surrounding it are feeble and virtually invisible in a microscope.

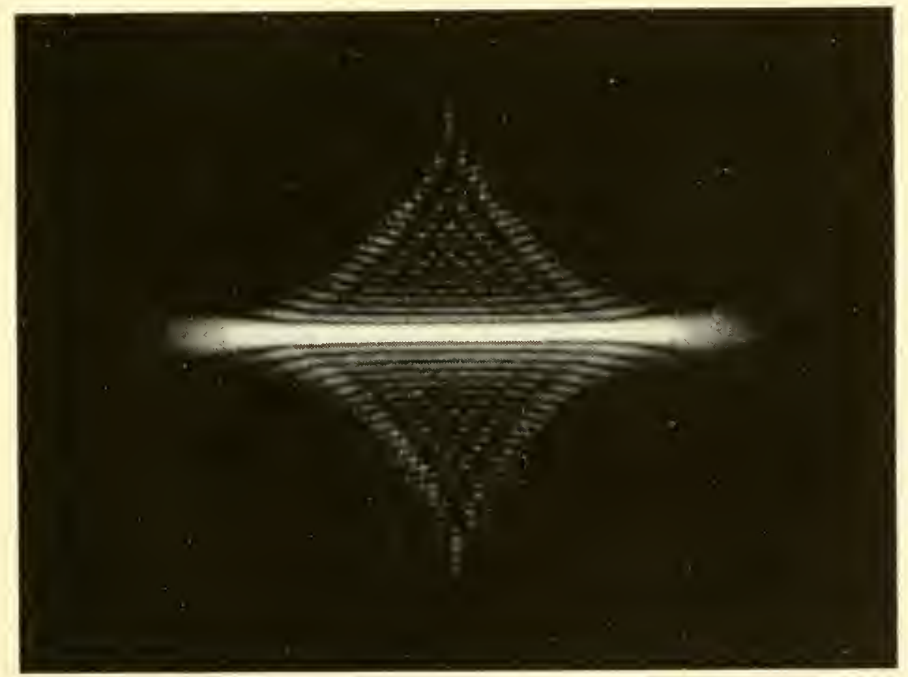

FIG. 1.26. Diffraction fringes surrounding a focal line (astigmatism).

The geometrical aspect of the problem prevails here. Both spherical aberration and coma must be considerable in order to cause the geometrical aspect of the phenomena to be evinced. This is the reason 
why the geometrical aspect of these two aberrations are never observed in microscopy. Their presence is only detected by slight alterations of the diffraction pattern. On the other hand, slight astigmatism affects substantially the diffraction pattern which vanishes and the focal lines of geometrical optics are evinced. Astigmatism is a common aberration in microscopy and is readily observed with high numerical aperture and low-magnifying objectives. It is advisable to ascertain that the astigmatism observed is not originated by the eyepiece which is

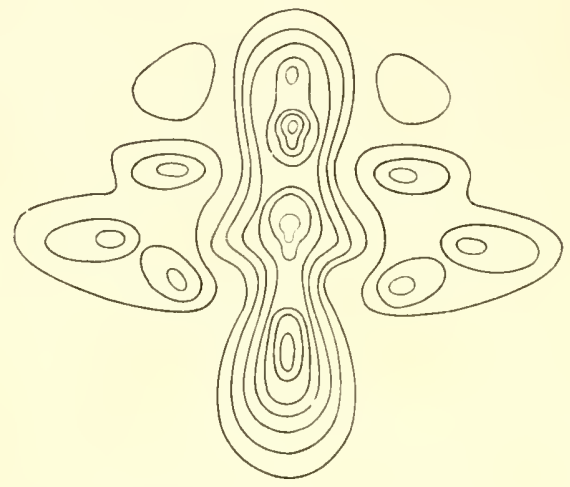

FIG. 1.27. Isophotes when spherical aberration, coma and astigmatism are present (after A. Marechal).

a common occurrence. While coma and astigmatism are tolerable on the edges of the field of view such is not the case for the centre because such aberrations would be due to the lens objective's being out of line. Such an objective is to be definitely rejected. When coma and astigmatism are present concurrently, the isophotal curves in the diffraction pattern may take on rather involved shapes. Figure 1.27 is a typical example of such a combination of aberrations.

\section{OB.JECTS IN COHERENT, INCOHERENT AND PARTIALLY COHERENT ILLUMINATION}

Optical objects termed "incoherent" are objects the various elements of which emit fully incoherent vibrations, i.c. independent from one another. Such objects, luminous per se, such as the sun, the various terrestrial sources, landscapes, are incoherent objects. The various monochromatic radiations forming the spectrum from a source are incoherent from one another. How emission of luminous vibrations takes place is still an uncompletely solved problem but it is known 
that energy exchanges between matter and radiation (electromagnetic waves) occur by quanta of value $h$ ( $h=$ Planck's constant, $v=$ frequency of the vibration emitted). It is usually considered that electromagnetic waves are emitted by wave-trains generated from the various atoms of the source.

Atoms emit for a very short time $\tau$. If a long time elapses in relation to $\tau$, as is always the case with our experiments, the initial vibrations shall have vanished. Other atoms will emit new vibrations having no relationship with the initial ones since their direction, amplitude and phase have changed. The various elements of a source are therefore incoherent.

Let us first consider the well-known experiment of Young's twin apertures. The latter (Fig. 128 ), $A$ and $B$, are identical and illuminated by the very minute source $S_{1}$. Interference fringes are seen on the screen $E$. Notwithstanding the very minute dimensions of the source $S_{1}$, the vibrations it emits are originated by a large number of atoms each of which emits at a given moment a vibratory motion unrelated to

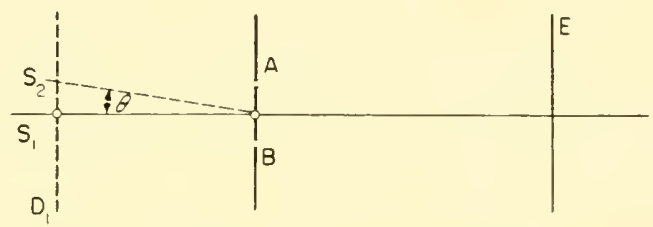

FIG. 1.28. Young's experiment.

those emitted by the other atoms of $S_{1}$. However, every atom-emitted vibratory motion gives rise to vibrations that reach $A$ and $B$ in the same condition because, provided $S_{1}$ be sufficiently small, it can be said that, from all its points, the optical paths $S_{1} A$ and $S_{1} B$ are equivalent. Phenomena occur as if apertures $A$ and $B$ emit coherent vibrations. Therefore the contrast of fringes is maximum on the screen. If $A$ and $B$ are illuminated by the second source $S_{2}$ alone, it would show well-contrasted fringes on the screen $E$ that, however, would be offset in relation to the interference fringes originated by $S_{1}$. Let us assume that sources $S_{1}$ and $S_{2}$ are illuminating $A$ and $B$ concurrently. Since both sources are incoherent, the resulting intensity, at any point of the screen $E$, is the sum of the intensities generated by both phenomena. When the angle $\theta$ is very small, offset of the two phenomena is negligible: apparently the latter 
occur as if source $S_{1}$ was the only one although the phenomenon seen on the screen is naturally brighter.

$A$ and $B$, therefore, can be illuminated with a large source in the plane $D_{1}$ without altering the visibility of the fringes, provided that the angle $\theta$, at which the source is seen from $A$ and $B$, be small ( $\theta$ must be small with respect to the interval between two fringes as seen from $A$ or $B$ ). When such is the case, from the standpoint of image contrast phenomena occur as if apertures $A$ and $B$ diffract coherent vibrations.

If the angle $\theta$ is increased, staggering of the various fringe systems due to the various elements of the source becomes prominent; phenomena do not coincide any longer, are blurred to a lesser or greater degree and contrast is decreased. If the angle $\theta$ is increased still further, a point is reached when phenomena are completely blurred, contrast is zero and fringes vanish. The two apertures $A$ and $B$ act as two incoherent sources and, of course, no interference can take place.

Let us now consider the next experiment (Fig. 1.29). The pin-point source $S_{0}$ is imaged on the plane $P$ by means of the lens $C$. Assuming,

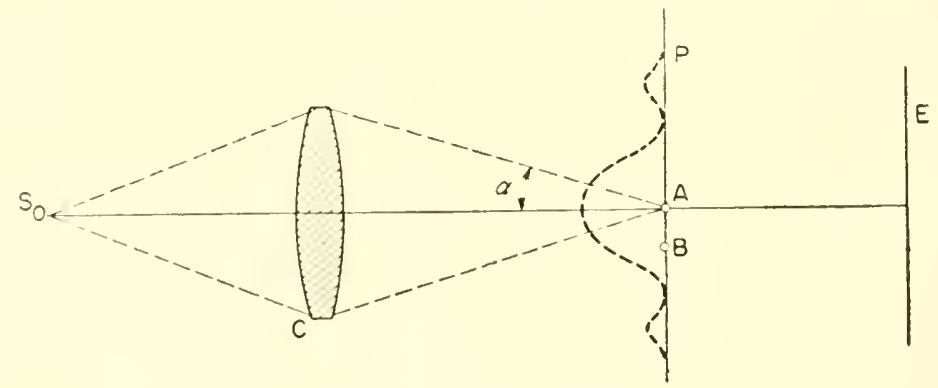

FIG. 1.29. Points $A$ and $B$ are illuminated in coherent light.

first, that aperture $\alpha$ of the lens $C$ be very small and, hence, the image of $S_{0}$ a well spread-out diffraction disk. Let points $A$ and $B$ (in plane $P$ ) be close enough to be within the central diffraction disk. Under such conditions, both theory and experimentation show that points $A$ and $B$ are illuminated in coherent light. Were $A$ and $B$ to consist of two holes pierced in the plane $P$, they would produce interference fringes on screen $E$ as in the previous experiment (Young's apertures). Let us increase the angle $\alpha$, viz. aperture of the lens $C$. The diffraction disk shrinks more and more and no alteration is evidenced if the distance from $A$ to $B$ is reduced adequately. The range within which $A$ and $B$ 
are coherent, the "range of coherence", merely shrinks. Without altering $\alpha$, let us take a large source (Fig. 1.30). This figure shows the diffraction disk related to the source centre $S_{0}$ and the diffraction disk produced by any point $S_{1}$. If the latter is close to $S_{0}$, both diffraction disks are slightly offset. Both points $A$ and $B$ are illuminated

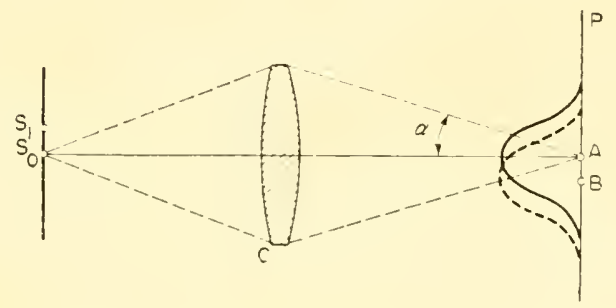

FIG. 1.30. Range of coherence with an extended source.

in coherent light by $S_{0}$ and $S_{1}$ too, but the two latter are incoherent. In both cases, $A$ and $B$ can produce interference fringes. The two fringe systems are incoherent but, as they are superimposed, nothing is altered. As point $S_{1}$ recedes from $S_{0}$, phenomena remain unaltered until the two diffraction disks are offset to such an extent that neither of points $A$ and $B$ are illuminated by $S_{1}$. Consequently, if a large source is imaged on the plane $P$ by means of the lens $C$, the two points $A$ and $B$ are always illuminated in coherent light provided that

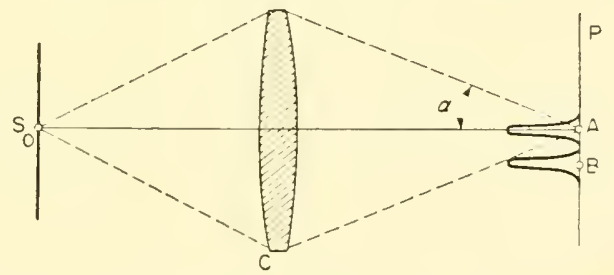

FIG. 1.31. Points $A$ and $B$ are illuminated in incoherent light.

the distance $A B$ be small in relation to the diffraction-disk diameter of $C$ (diameter depending solely on $\alpha$ ). When distance $A B$ is greater (Fig. 1.31) the two points $A$ and $B$ are no longer overspread by the same diffraction disk in relation to a point of the large source and, therefore, are illuminated in incoherent light. Hence, the coherence in relation to two points $A$ and $B$ in the plane $P$ depends solely on the aperture $\alpha$ of the lens $C$. 
Before going into these phenomena as they occur in a microscope, the two methods of illuminating a specimen will be set forth. In the Köhler method, the source $S_{0}$ is imaged by means of the lens $L$ on

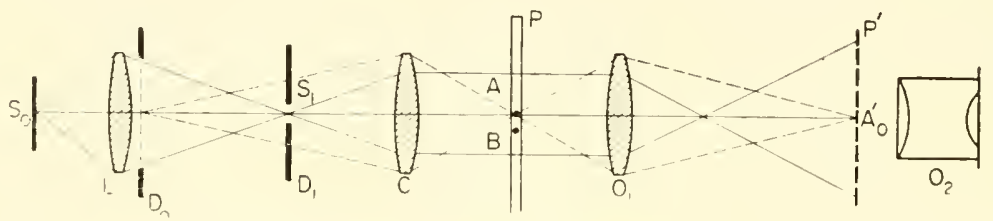

FIG. 1.32. Köhler illumination.

the iris diaphragm $D_{1}$ of the condenser $C . S_{0}$ and $L$ form the illuminator of the microscope and may be either separate or integral with the instrument.

The specimen is at $P$; the objective $O_{1}$ images it at $P^{\prime}$ where it is observed through the eyepiece $O_{2}$. Against the lens $L$ is the iris diaphragm $D_{0}$. The position of the condenser $\left(C\right.$ and $\left.D_{1}\right)$ is adjusted so as to image the diaphragm $D_{0}$ on the specimen $P$. Next, $D_{0}$ is diaphragmed to the extent that only the field actually observed is illuminated. In this way, spurious light is reduced to a minimum because even the specimen areas outside the visible field may transmit light within the microscope. Some of this light always reaches the image and the resulting halo weakens contrasts.

Figure 1.33 shows the other method of illumination: the condenser $C$ images the source $S_{0}$ into the specimen $P$. Figure 1.33 is simplified

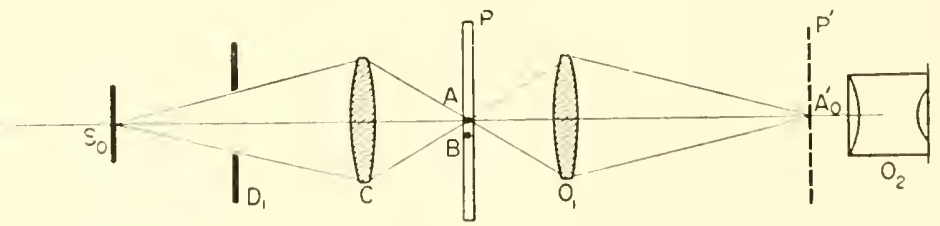

FIG. 1.33. Critical illumination.

because, by means of an additional optical system and another diaphragm, only the specimen field actually observed need be illuminated, as in the Köhler method. But this method may result in uneven illumination and show, superimposed on the object image, a spurious image possibly exhibiting luminance variaitons.

However that may be, theory shows that both Köhler and critical illumination take effect, from the coherence standpoint, in exactly 
the same way. As critical illumination is a more convenient basis for discussion, it will be used to investigate image coherence as it obtains in microscopy.

(a) The mumerical aperture of the condenser is very low

It is assumed that the aperture of the condenser iris diaphragm is minimal. Under such conditions, every point of the source $S_{0}$ (Fig. 1.34) gives rise to a diffraction disk overspreading substantially

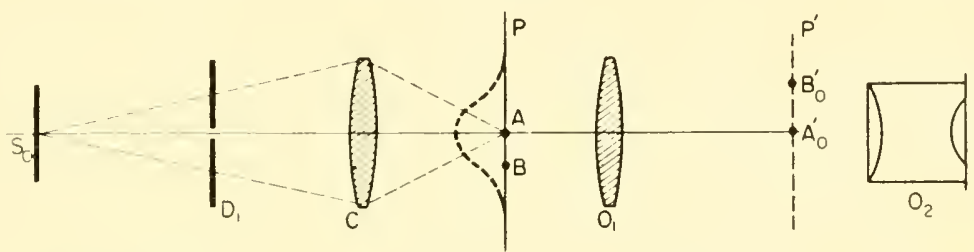

FlG. 1.34. Points $A$ and $B$ are within the condenser central diffraction disk.

in the plane $P$ of the specimen. In Fig. 1.34, the diffraction disk shown is correlated to a point of the extended source. Let us consider two pin-point details $A$ and $B$ in the specimen, close enough to be within the condenser-originated central diffraction disk. Under the effect of the incident light, $A$ and $B$ diffract coherent vibrations. Irrespective of the objective's numerical aperture $n \sin u$, images $A_{0}^{\prime}$ and $B_{0}^{\prime}$ of $A$ and $B$ are coherent diffraction disks. When $n \sin u$ is also very low, $A_{0}^{\prime}$ and $B_{0}^{\prime}$ are not discrete and these two points exhibit two diffraction disks merging at $P^{\prime}$. This instance is obviously not very useful in microscopy since the relevant objectives do not have very low numerical apertures. If $n \sin u$ increases, the images $A_{0}^{\prime}$ and $B_{0}^{\prime}$ may be separated although remaining, naturally, coherent. Therefore, when considering an extended object of high contrast whose dimensions are smaller than the diameter of the condenser-originated diffraction disk, it is seen in coherent illumination.

(b) The numerical aperture of the condenser is random but not low

Investigation is restricted to a high-contrasted object, but smaller than the diffraction disk of the objective. Let us take diaphragm $D_{1}$ of the condenser (Fig. 1.35) so that the diameter of the condenser-originated central diffraction disk is definitely smaller than distance $A B$. Both points $A$ and $B$ are overspread by two diffraction disks from two different points of $S_{0}$. The points $A$ and $B$ are incoherent and emit 
incoherent vibraitions. Let us assume that the numerical aperture $n \sin u$ of the objective is much lower than the condenser's. The central diffraction disk of the objective is wide (curve $U$ ) and overspreads in width the diffraction disks of the condenser at $A$ and $B$. The intensities shown are naturally random. The purpose sought is merely to compare the diameters of the central disks. When points $A$ and $B$ are integral with a small object of high contrast whose size is less

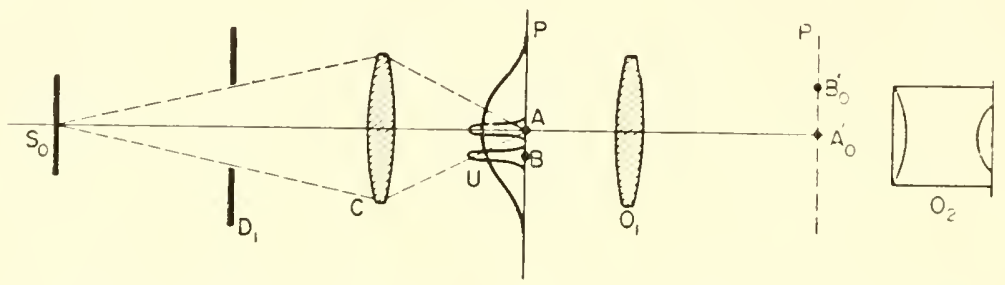

FIG. 1.35. The diameter of the condenser central diffraction disk is smaller than distance $A B$.

than the diameter of the objective's central diffraction disk, the object is seen in incoherent light. Such a case is not met with in microscopy as, in order to have images of high-contrasted objects in incoherent light, the objective's numerical aperture has to be so much lower than the condenser's that. when normal apertures are used in the latter, this is tantamount to using an objective of very low numerical aperture.

Diffraction disks are of the same magnitude when the N.A. of both objective and condenser are somewhat similar. Now, since we are considering an object of smaller size than the diameter of the objective's central diffraction disk. this means that points $A$ and $B$ are not to be considered as being as far apart as previously. The points $A$ and $B$ to be selected must be closer so as to be included within the central diffraction disk correlated to the objective. The condenser N.A. selected is usually lower than the objective's. The central diffraction disk of the condenser is thus wider than the objeclive's and the conditions shown in Fig. 1.34 are reverted to. Illumination is virtually coherent.

Thus far no mention has been made regarding condenser defects. Theoretical considerations show that its aberrations take no effect on image coherence which depends solely on the condenser aperture.

Other reasons militate in favour of a high-cuality condenser: Köhler's method provides a sharp image of the diaphragm $D_{0}$ on 
the specimen (Fig. 1.31). Areas not within the field of view are unilluminated and spurious light is minimized. Lastly a corrected condenser (aplanatic) provides the evenly illuminated field, indispensable in photomicrography.

To sum up it can be said that for objects of high contrast and smaller than the central diffraction disk of the objective, images are virtually in coherent illumination. Stich is the case of the more valuable details observed in a microscope.

Naturally, coherence varies somewhat when the condenser aperture is altered and, even when close to small-object coherent illumination. differences in appearance are developed whose effects are far-reaching and will be discussed later.

For objects of low contrast the phenoma are not the same. Coherence varies much more quickly. For a small black disk, illumination is again virtually coherent when apertures of the objective and condenser are equate. Under the same conditions, illumination is incoherent for an object of low contrast.

In general, incoherent illumination does not come in microscopy except for the object of low contrast and in fluorescence. In the latter case the specimen is illuminated by ultraviolet light and emits visible light. The various points of the specimen are then incoherent.

\section{EXTENDED OBJECTS IMAGED IN INCOHERENT ILLUMINATION}

When considering the extended object $P$ (Fig. 1.36), it may be broken up into small luminous elements acting as discrete pin-point sources. Thus the object $P$ can be considered as consisting of minute

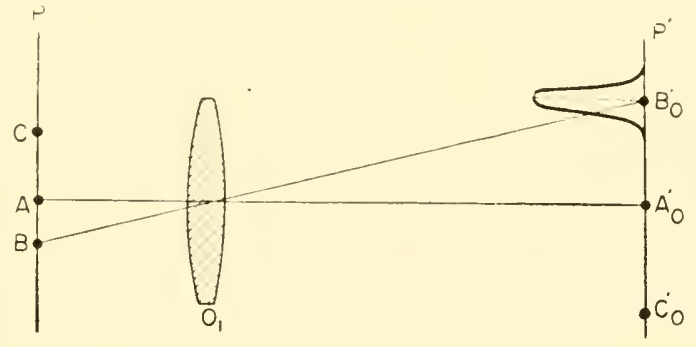

FIG. 1.36. Image formation of an extended object.

luminous sources $A, B, C$, etc. that can be likened to real pin-point sources. The object $P$ is seen in incoherent illumination provided its constituent sources $A, B, C$ act as thoroughly independent sources 
whose vibratory motions are totally unrelated. To every constituent source, e.g. $B$, corresponds the diffraction disk $B_{0}^{\prime}$. The image $P^{\prime}$ of the object $P$ is the outcome of the superimposition of all diffraction disks the centres of which are $A_{0}^{\prime}, B_{0}^{\prime}, C_{0}^{\prime}$, etc. It follows that intensity in the centre of a diffraction disk depends on the light-intensity of the relevant object point lines. In areas where the object is dark, the constituent sources transmit but little light and the relevant diffraction disks are likewise but slightly illuminated. To better illuminated areas correspond better illuminated diffraction disks. The image $P^{\prime}$ is achieved by summing all the intensities originated by all the diffraction disks. Figure 1.37 shows the imaged plane $P^{\prime}$ of object $P$.

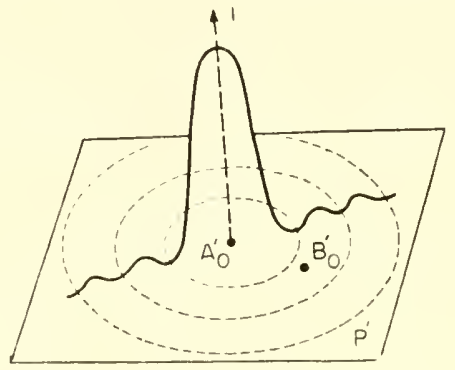

Fig. 1.37. Image formalion of an incoherent extended object.

Required: to determine the light-intensity at any point $B_{0}^{\prime}$ of the image. Were only the source $B$ involved, the intensity at $B_{0}^{\prime}$ would merely be the peak ordinate of the $B_{0}^{\prime}$ diffraction disk. If the source $A$ takes effect simultaneously, the light diffracted by $A_{0}^{\prime}$ and reaching $B_{0}^{\prime}$ must be taken into account. In fact the image $A_{0}^{\prime}$ is a diffraction disk extending in the plane $P^{\prime}$. To determine the light-intensity at $B_{0}^{\prime}$, originated by the sources $A$ and $B$, requires summing together the intensity originated by the source $B$ (peak ordinate of $B_{0}^{\prime}$ centre diffraction disk) and the intensity originated by the light diffracted by the image $A_{0}^{\prime}$. Effect of $A_{0}^{\prime}$ on $B_{0}^{\prime}$ increases as distance $A_{0}^{\prime} B_{0}^{\prime}$ is short in relation to the diffraction-disk size. To determine the structure of the whole $P$ image the operation is resumed for all the points of the image $P^{\prime}$, taking into account the relevant intensities of the object points. Thus is derived the distribution of imaged light-intensities. The structure of images can be derived mathematically. In many cases a deft application of the Fourier transformation enables one to calculate the phenomena in a comparatively simple manner. Owing to the 
multifariousness of images met with in microscopy, the results shown refer to typical objects similar in nature to the basic details of microscope specimens frequently met with.

Small black disk on white ground imaged in incoherent illumination

The object now considered is a black disk, small in relation to the objective-originated diffraction disk. Figure 1.38 shows the distribution of light-intensities in accordance with image diameter. The pecked line curve is related to the geometrical image of the object. Intensity

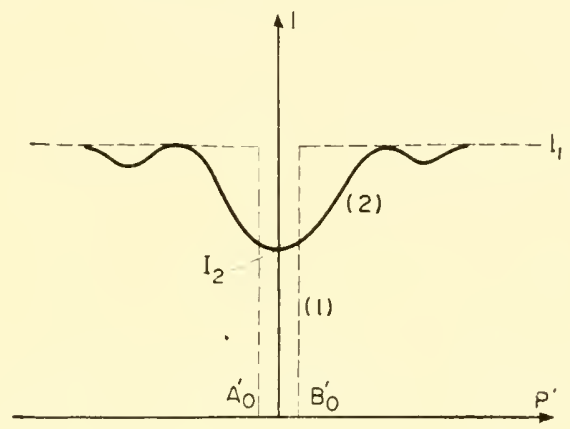

FIG. 1.38. Small black disk on white ground, imaged in incoherent illumination.

is zero within a circumference of diameter $A_{0}^{\prime} B_{0}^{\prime}$ (diameter of the geometrical image of the dark disk). Outside of this area, field illumination is uniform, its intensity being, e.g. $I_{1}$. Curve 2 shows the intensity distribution in the actual image of the dark disk. Owing to diffraction, the intensity $I_{2}$, in the image centre, is not zero; there are no breaks and the image is wider. The image contrast may be defined by the expression:

$$
\gamma=\frac{I_{1}-I_{2}}{I_{1}}
$$

If $I_{2}=0$, i.e. the image centre is black, the contrast is:

$$
\gamma=1 \text {. }
$$

The image contrast equates the contrast of the object itself and is then maximum. The contrast dwindles as $I_{2}$ increases. There is no contrast when $I_{2}$ equates $I_{1}$. Contrast values are, therefore, included between the minimum value 0 and the peak value 1 . In all the following applications, it is assumed that the observer's eye functions as if it was a perfect instrument. In normal daylight 
vision the eye is considered as a perfect instrument provided it functions with a pupil of approximately $0.5 \mathrm{~mm}$ in diameter. Such a diameter does not tally with an eye pupil but with an artificial pupil originated by the microscope itself as happens when the exit pupil of the microscope has a diameter of $0.5 \mathrm{~mm}$ (optimum magnification). Then the light entering the eye is restricted to a $0.5 \mathrm{~mm}$ diameter circle which acts as pupil: the eye functions as a perfect instrument featured by a single physiological datum: the minimum contrast perceivable. In the case of a small, imaged dark disk, the lowest contrast a normal eye can perceive is approximately 0.04 , for most other objects this figure drops to 0.02 . Owing to diffraction, contrast of the imaged dark disk varies as its radius $r$. As $r$ decreases, so does contrast and when $\gamma=0.04$ the image vanishes (for a small dark disk on a white ground). This obtains when the radius $r$ of the black disk is educed from the following expression:

$$
r=\frac{0 \cdot 13 \lambda}{2 n \sin u}
$$

For instance, with an objective of numerical aperture $n \sin u=1 \cdot 30$ and a mean wave-length value $\lambda=0.6 \mu$, then $r=0.03 \mu$.

Formula 1.4 implies that $r$ is small in relation to the diffraction disk. Image of a small white disk on black ground

Merely reversing Fig. 1.38 will show the image of a white disk on black ground. The pecked-line curve 1 (Fig. 1.39) shows the

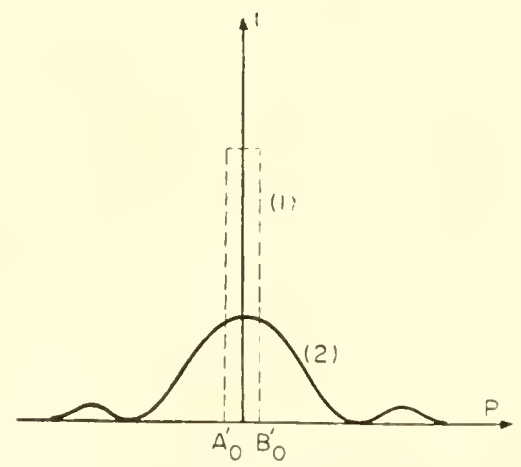

FIG. 1.39. Image of a small white disk on black ground.

intensity distribution in the geometrical image of the white disk and curve 2 the actual image, taking diffraction phenomena into consideration. As the disk with respect to the diffraction pattern is of 
small diameter, the image is virtually Airy's disk, whether coherently or incoherently illuminated.

Perception of a small white disk on black ground is a problem which has not the same aspect as that of a black disk on white ground. As the diameter of a small black disk shrinks, ingress of diffracted light increases until the image is completely blotted out. When the object is a small white disk on a black ground and its diameter decreases, the ultimate image is the conventional diffraction disk (Airy's disk). If the disk still dwindles in size, the curve showing the intensity distribution in the diffraction disk gradually gets flatter but the lateral dimensions of the diffraction disk remain constant. Visibility of a small white disk on black ground is, therefore, merely a matter of luminous flux. The smaller the disk, the more light it must send forth.

\section{Black line on white ground imaged in incoherent illumination}

Now the object is a thin, long black line. It follows that lightintensity is constant along a line parallel to the thin line and the information required is merely the light-intensity changes taking place along a straight line at right angles to it. The curves in Fig. 1.40 show

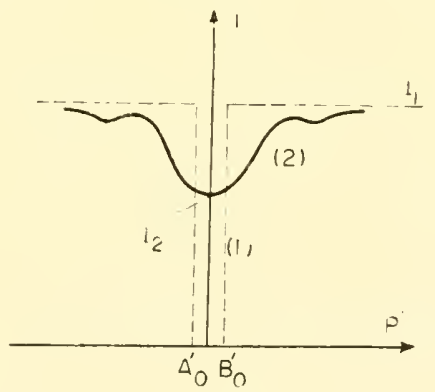

FIG. 1.40. Black line on white ground imaged in incoherent illumination.

the general aspect of the phenomena involved. The geometrical width of the imaged black line is shown by $A_{0}^{\prime} B_{0}^{\prime}$. Curve 2 shows how intensity is distributed in the imaged black line: this curve does not extend to the horizontal line anywhere. The lowest contrast perceivable to the eye for an object of this type is 0.02 . As with the black disk, there is a relationship between the contrast $\gamma$ of the imaged black line and its width $\varepsilon$. As the width of the line decreases so does the contrast until the image contrast has dropped to 0.02 , and the line has reached 
the limit of perception. Such is the case when

$$
\varepsilon=\frac{0.02 \lambda}{2 \pi \sin u} \text {. }
$$

For instance, with a 1.30 N.A. objective and $\lambda=0.6 \mu$, then $\varepsilon=0.005 \mu$. Naturally, as in the previous example, this theoretical result applies only to an aberration-free instrument.

Luminous thin streak on black ground imaged in incoherent illumination

This streak is educed at once from the image of the thin, black line. The distribution of light-intensities in the image of a thin streak of light on a black ground is shown by curve 2 in Fig. 1.41. There are

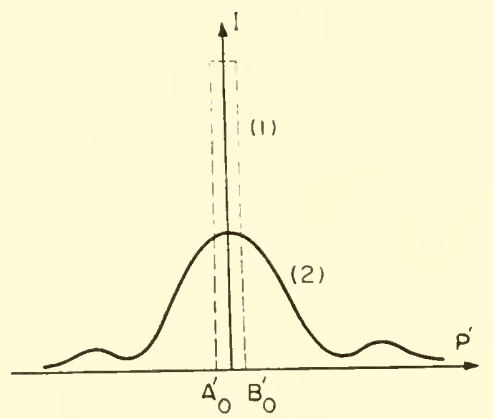

FIG. 1.41. Luminous thin line on black ground imaged in incoherent illumination.

no zero minima in the diffraction pattern. As with the small luminous disk on black ground, the problem of descrying a thin streak of light on a black ground is merely a matter of luminous flux; to be perceived. a very thin streak of light must emit enough of it.

Area edge imaged in incoherent illumination

Let us consider as object an illuminated half-plane, separated from the black area by a straight line (Fig. 1.42). The pecked curve 1 (Fig. 1.43) shows the structure of the geometrical image along a parallel

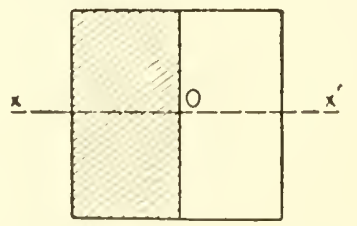

FIG. 1.42. Illuminated half-plane object. 
line $x^{\prime}$ (Fig. 1.42), and curve 2 (Fig. 1.43) shows the light-intensity distribution in the image in the presence of diffraction, which also does away with any sudden break in intensity when changing over from the imaged illuminated area to the black one. Shaded-off

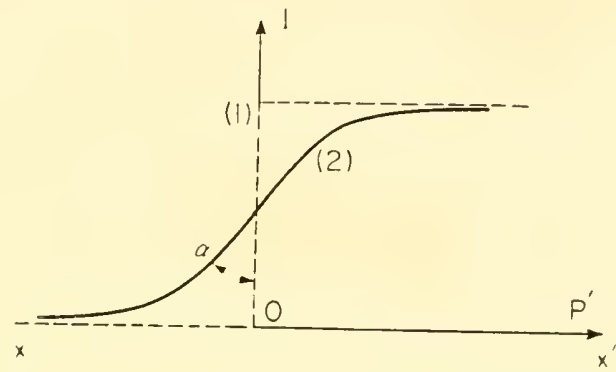

FIG. 1.43. Area edge imaged in incoherent illumination.

illumination straddles the geometrical boundary and extends on either side of it but soon becomes hardly perceptible. The smaller the angle $a$ the sharper the image outline.

Periodic object imaged in incoherent illumination

Let us take as periodic object a Foucault test divided into alternately light and dark parallel lines of identical length and width (Fig. 1.44). Such an object can be likened to some periodic structures occasionally

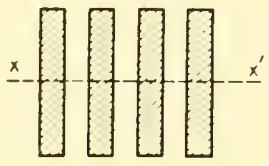

FIG. 1.44. Periodic object (Foucault test).

met with in microscopy. If the dark lines are absolutely black as in Fig. 1.45, the contrast equates unity. If not (Fig. 1.46) the contrast decreases and may have any value from 1 to 0 . Let $I_{1}$ be the intensity

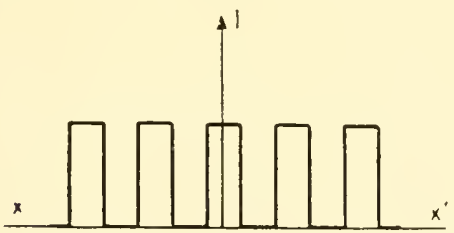

FIG. 1.45. Light distribution in the Foucault test.

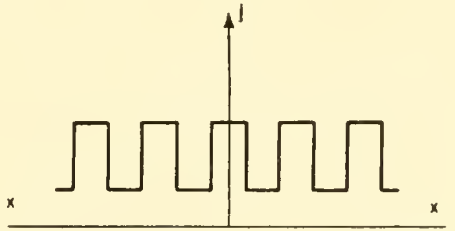

FIG. 1.46. Foucault test with contrast smaller than unity. 
of the bright lines and $I_{2}$ that of the dark ones. As with the previous objects, the contrast can be determined by the equation (1.3). In the image plane, intensity distribution has the shape shown by curve 2 in Fig. 1.47. Curve 1 shows the geometrical image. Therefore, the phenomenon obtained is always the same: there are no breaks; outlines are smoother and contrast is decreased.

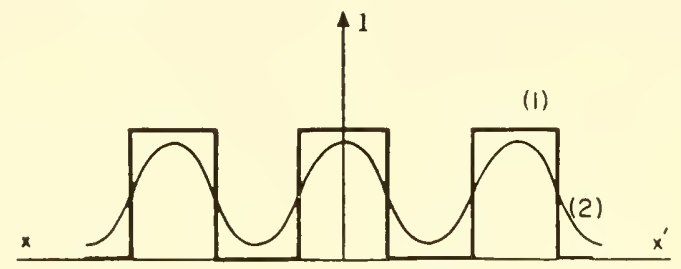

FiG. 1.47. Foucault test image - (1) geometrical; (2) actual image.

Theory shows that a Foucault test of the type depicted in Fig. 1.45 can be shown by superposing any number of sinusoids, similar to those in Fig. 1.48, the periods of which are $p, \frac{1}{2} p, \frac{1}{3} p$, etc. It follows that the instrument transmits the components of long periods (spacedout lines) much better than those of short periods (lines close together). Applying the term "frequency" to the converse of period $p$, it can be said that an instrument always conveys the low-frequency components

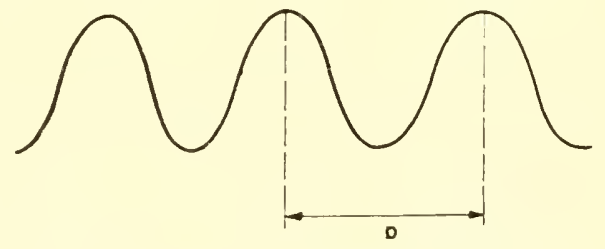

FIG. 1.48. Sinusoid the period of which is $p$.

better than the high-frequency ones, which is tantamount to saying that the closer together the elements of a periodic structure the less resolvable they are. Educing how such frequencies are transmitted by a specific instrument is desirable. The curve (Fig. 1.49) shows the results. Frequencies $1 / p$ are plotted as abscissace while the contrast factor, the parameter $M$, which determines how every frequency is transmitted by the instrument, is plotted as ordinates. If $M=1$, the frequency considered is transmitted faultlessly; if $M=0$, it ceases. At very low frequencies, the contrast factor is virtually equivalent to 1 and, hence, they are well transmitted. As the frequency rises, viz. when $p$ 
drops, so does the transmission of the sinusoidal object. The transmission becomes zero when $1 / p=(2 n \sin u) / \lambda$ (the period $p$ being measured in the object plane). The instrument does not transmit

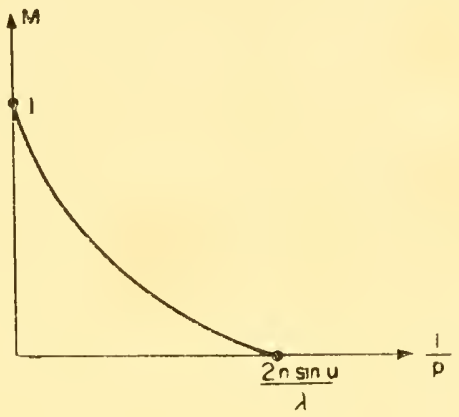

FiG. 1.49. Contrast factor of an optical instrument.

frequencies exceeding $(2 n \sin u) / \lambda$; the object-rod can therefore be distinguished only if its bars are spaced by $p$, as follows:

$$
p \geqslant \frac{\lambda}{2 u \sin u} \text {. }
$$

The equation (1.6) is tantamount to showing the instrument's resolving power. Therefore, with a test of the type shown in Fig. 1.45, the period of which is $p$, when resolving power is reached, the imaged test is a sinusoidal image exhibiting the same period whose value is derived from the above equation. For instance, with a 1.30 N.A. objective and $\lambda=0.6 \mu$ a periodic structure $p$ of at least $0.23 \mu$ can be perceived in incoherent illumination.

\section{EXTENDED OBJECTS IMAGED IN COHERENT AND PARTIALLY COHERENT ILLUMINATION}

Referring to Köhler illumination (Fig. 1.50), let us diaphragm down almost completely the condenser lens $D_{1}$. The light source from the very small aperture $S_{1}$ forms a cylindrical pencil of parallel rays that passes through the specimen $P$ and penetrates into the objective $O_{1}$. Owing to the direct light from $S_{1}$ a small detail $A$ diffracts some of it. The wider the angle $\theta$, at which the light is diffracted by $A$, the smaller the detail considered. The light diffracted by $A$ is collected by the objective $O_{1}$ and converges on $A_{0}^{\prime}$. The image $A_{0}^{\prime}$ of the detail $A$ results from superposing the coherent ground $B^{\prime} C^{\prime}$ and the 
light diffracted by $A$ which ends at $A_{0}^{\prime}$. This time, however, these two illuminations, the coherent ground and the diffracted light, although not independent from the vibratory standpoint since they are originated by the same pin-point source $S_{1}$, are coherent. Hence it is not both ground and the diffracted light-intensities that are to be added together

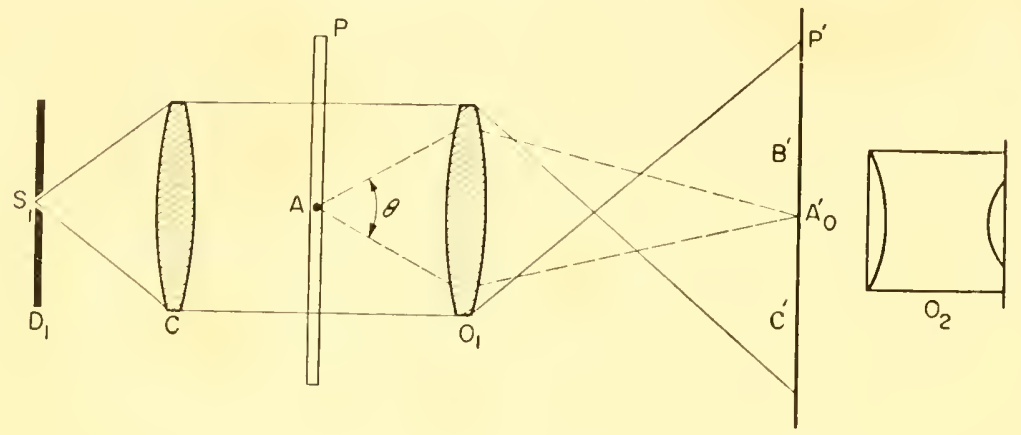

Fig. 1.50. Extended object $P$ in coherent illumination.

but those of the amplitudes of the luminous vibrations reaching $A_{0}^{\prime}$, taking into account the path difference or the phase difference of these vibrations. When the condenser diaphragm $D_{1}$ is opened, illumination becomes partially coherent and the image structure is usually difficult to calculate.

Small black disk on white ground imaged in coherent and partially coherent illumination

Let us first consider a small black disk imaged in coherent illumination, i.e. when $D_{1}$ is almost fully diaphragmed down. Results are shown by curve 2 (Fig. 1.51). The structure is similar to that shown in Fig. 1.38 but the fringes are much more prominent. Figure 1.52 conveys much information: it shows how the image

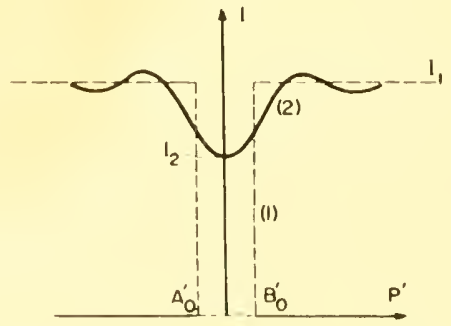

FIG. 1.51. Small black disk on white ground imaged in coherent illumination. 
evolves in accordance with the aperture of the condenser. Ordinates show the light intensities taking as unit the intensity at a point far removed from the imaged black disk; abscissae show the distances to the imaged geometrical centre. The geometrical width of the imaged black disk is shown by the distance included between the two small vertical lines above the curves in Fig. 1.52. When $D_{1}$ is almost fully

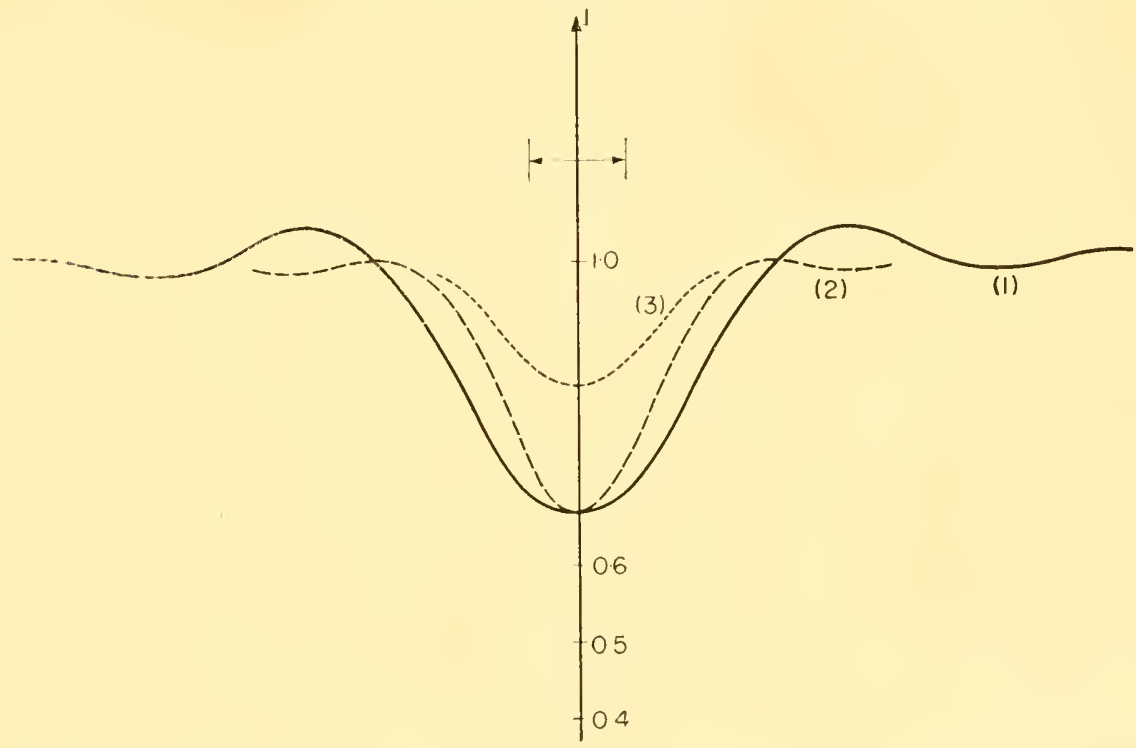

FIG. 1.52. Image of a small black disk in relation to the aperture condenser: (1) coherent illumination; (2) partially coherent illumination; (3) incoherent illumination.

diaphragmed down (coherent illumination) curve 1 shows the result. When the diaphragm aperture is increased (partially coherent illumination) so as to impart to the condenser a N.A. equivalent to that of the objective, curve 2 shows the result. When illumination is incoherent, curve 3 shows the result.

These curves enable one to state the following: as the condenser N.A. is increased, the clearer the image, the less prominent the diffraction fringes, without this virtually entailing a change in contrast of the image. Moreover, in order to prevent stray light, the condenser has a N.A. lower than that of the objective. Therefore it can be said that the image contrast of a small object, in microscopy, always equates the image contrast in coherent illumination. Since diaphragming down $D_{1}$ leads to extending the image to the detriment of its quality, it follows that it is best to open suitably the diaphragm $D_{1}$, 
i.e. to increase the condenser N.A. so as to lessen blur as much as possible. The curves in Fig. 1.52 correlate the results set forth in paragraph 7 , and show that the image contrast of a small disk in coherent illumination is improved 2 to 1 . If $r$ is the radius of the black disk, then:

$$
r-\frac{0.08 \lambda}{2 n \sin u}
$$

In order to determine the perception of a small detail, it is this formula which should be used in microscopy since, regardless of condenser N.A., and under normal observation conditions, phenomena occur as if illumination were coherent. As an instance, with a 1.30 N.A. objective and $\lambda=0.6 \mu, r=0.02 \mu$. This theoretical result implies, naturally, a perfect objective.

Let us now revert to the examples of achromatic, fluorite and apochromatic objectives mentioned in $\$ 5$. The chromatic effect of these three types of objectives on the contrast of an imaged small dark disk can be assessed as follows: using the same objectives as previously, it is found that the contrast of a small disk (radius $0.08 \mu$ ) is 0.33 for a perfect objective, 0.31 for the apochromatic objective, 0.27 for the fluorite objective and 0.18 for the achromatic objective (focusing wave-length $=0.56 \mu$ ). The contrast achieved with an apochromatic objective is improved 2 to 1 thus making much smaller details resolvable.

\section{Inage of bright small disk on black gronnd}

When the disk is small in relation to the diffraction-disk radius, the image is a conventional diffraction disk (Airy's disk), whether illumination is coherent or incoherent. Image perception is then only a matter of luminous flux.

Image of thin black line on white ground in coherent or partially' coherent illumination

As with a small black disk Fig. 1.51 shows the general aspect of the results. As the line attains the perception limit $\varepsilon$ its width is derived as follows:

$$
\varepsilon=\frac{0 \cdot 01 \lambda}{2 n \sin u}
$$

The black-line contrast is improved 2.35 to 1 in coherent illumination compared with incoherent illumination. If $n \sin u=1.30$ and 
$\lambda=0.6 \mu, \varepsilon=0.002 \mu$. As with a small black disk, evolvement of the imaged line in terms of condenser aperture is shown in Fig. 1.52. An imaged thin line is always very similar to the image in coherent illumination and it is equation (1.8) that should be used in microscopy except when fluorescence is involved. As condenser aperture is increased, diffraction fringes become less prominent and the image becomes finer while retaining the same contrast.

Image of thin streak of light on black ground in coherent illumination

The image is not the same as in incoherent illumination. In coherent illumination, the diffraction-pattern minima are zero minima
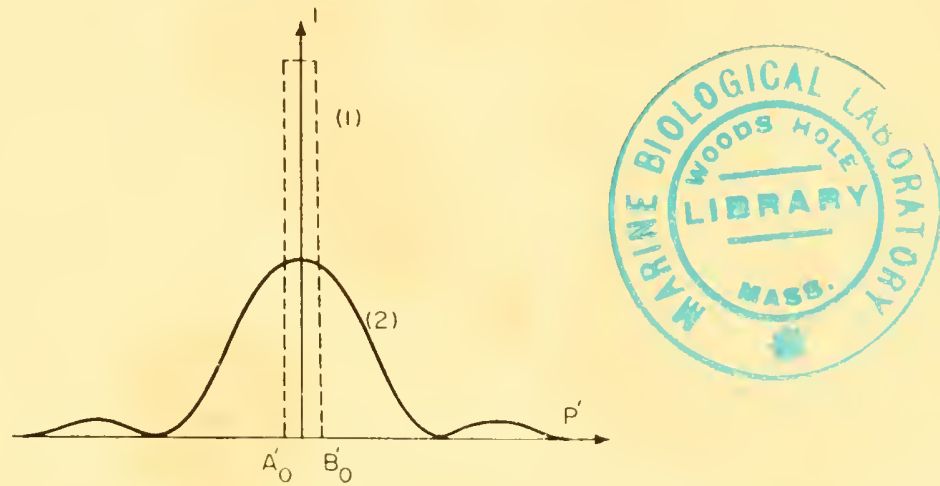

FIG. 1.53. Image of thin streat of light on black ground in coherent illumination.

(Fig. 1.53, curve 2). Perception of a thin streak of light on black ground is also a matter of luminous flux. Extremely fine streaks of light are perceivable, provided the luminous flux emitted by them be adequate.

Edge of area innaged in coherent and partially coherent illumination

Referring again to Fig. 1.42, the curves are those shown in Fig. 1.54. Curve 1 applies to coherent illumination, curve 2 to partially coherent illumination when the condenser N.A. is the same as the objective's. The diffraction fringes are clearly visible in coherent illumination and impair the image sharpness along the area edges. Increasing aperture of the condenser diaphragm causes these fringes to vanish.

Periodic object in coherent and partially coherent illumination

Referring again to the object shown in Fig. 1.44, the luminous lines consist of narrow parallel slits, evenly spaced and of equal size. 
Their number is assumed to be very large. Spacings between the slits are opaque and of the same width as the latter. This is, in fact,

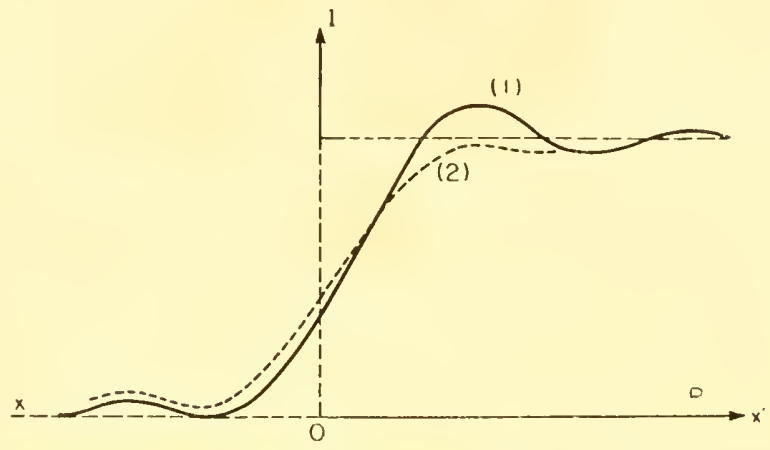

FIG. 1.54. Edge of area imaged in coherent and partially coherent illumination.

a grating which is substituted for the specimen $P$ and observed in monochromatic light (Fig. 1.55). Naturally, in Fig. 1.55, the slit width is unduly large. As the direct light from the source $S$ impinges against

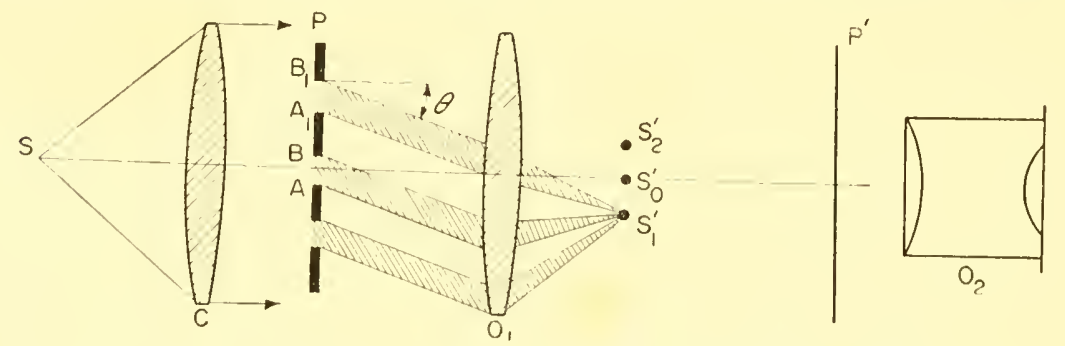

FIG. 1.55. Image of a periodic object in coherent illumination.

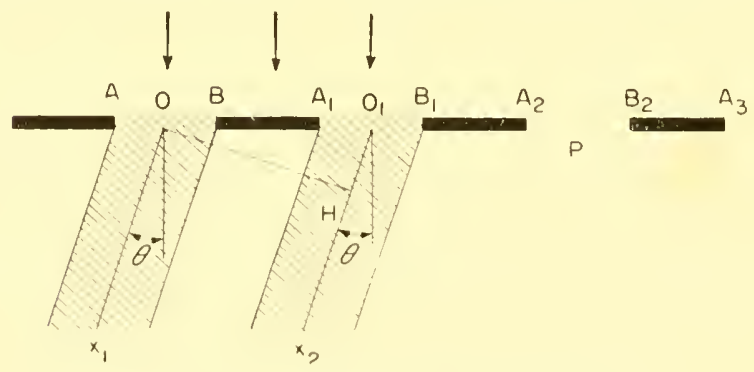

FIG. 1.56. Diffraction by parallel slits.

the object $P$, each slit, such as $A B$ or $A_{1} B_{1}$ diffracts some of the incident light. Let us consider the phenomena taking place at slit $A B$ and $A_{1} B_{1}$ (Fig. 1.56). The vibrations diffracted by the slit media $O$ 
and $O_{1}$ in a random direction $\theta$ do not travel along the same paths. Assuming the grating to be perpendicular in relation to the incident light-beam, points $O$ and $O_{1}$ are reached by the incident rays at the same time but the rays diffracted in the direction of 0 (vibrations originated by $O_{1}$ ) lag slightly in relation to those originated by $O$. Let us drop a perpendicular $O H$ from $O$ to $O_{1} x_{2}$. From $O$ and $H$ the vibrations diffracted by $O$ and $O_{1}$ travel together in the direction of $\theta$. The lag of the vibrations diffracted by $O_{1}$ equates $O_{1} H$. To every point of the slit $A B$ there is a matching point of slit $A_{1} B_{1}$ to which the same inference applies. At all points of slits $A B$ and $A_{1} B_{1}$ the phenomenon is identical with that at $O$ and $O_{1}$. Therefore all slits may be substituted for their centres.

The foregoing applies to the other slits too: the path difference increases or diminishes, from one slit to another, by the distance $O_{1} H$. The vibration diffracted in the direction of $\theta$ by slits $A_{1} B_{1}, A_{2} B_{2}$, $A_{3} B_{3}$, lag by $\lambda, 2 \lambda$, $3 \lambda$, etc., in respect of the vibrations diffracted by $A B$. As the phenomena recur identically within one wave-length or whole multiples thereof, nothing differentiates the slit-originated vibrations. Figure 1.57 shows there is no difference whatever between the vi-

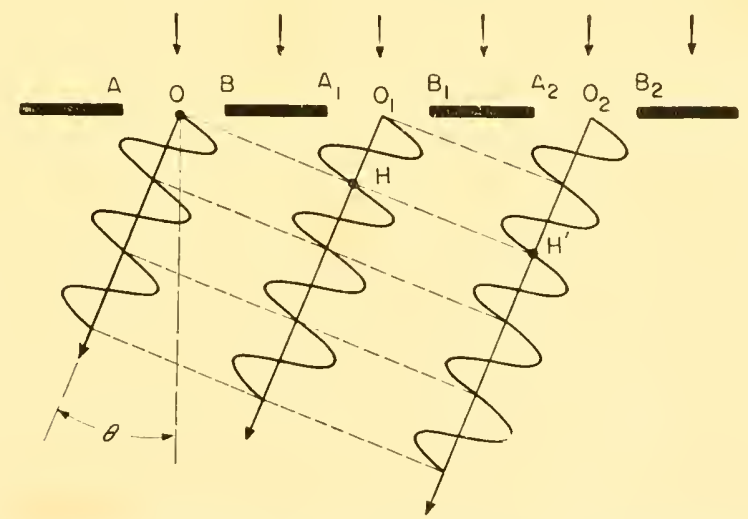

FIG. 1.57. The vibrations originated by the slits in the direction of $\theta$ are in phase.

brations $O, H, H^{\prime}, H^{\prime \prime}$, etc., and phenomena occur as if the vibrations originated by $O, O_{1}, O_{2}, \ldots$ were not out of phase. The vibrations diffracted by the slits in the direction of $\theta$ are in phase. Then, amplitude of the light emitted by the grating in that direction equates the sum of all the amplitudes emitted by the slits of the grating. After passing through the objective $O_{1}$ (Fig. 1.55) the maximum of light is produced in the focal plane at $S_{1}^{\prime}$. The rays diffracted in the 
direction of $\theta$ are concentrated in the focal plane of the objective $O_{1}$ because they are all parallel. Were $\theta$ changed to $-\theta$, i.e. were the phenomena considered to be symmetrical in relation to a perpendicular grating, nothing would be altered. The maximum of light would still obtain at $S_{2}^{\prime}$ and be symmetrical in relation to the axis. More particularly, were $O_{1} H=0$, viz. the light diffracted in the direction of the incident light $(\theta=0)$ investigated, the result would be the same. All the slit-originated vibrations in the direction of the axis $(0=0)$ are in phase and maximum light is produced at $S_{0}^{\prime}$, principal focal point of the objective $O_{1}$. The slit-originated vibrations are no longer in phase when there are slight divergences in the directions relevant to $O_{1} H=0, \lambda, 2 \lambda$. Some are leading, some lagging and, in the aggregate, they virtually cancel themselves out. There is virtually no light as soon as the directions, requiring a path difference amounting to a whole wave-length multiple, are diverged from. Lastly, when observing the focal plane of the objective $O_{1}$ (Fig. 1.55) the maximum of light is at $S_{0}^{\prime}$ on the axis and, symmetrically, on either side of $S_{01}^{\prime}$, a series of maximums $S_{1}^{\prime}$, $S_{2}^{\prime}$, etc. These maximums are images of the source. The image $S_{0}^{\prime}$ is the direct image of the source and images $S_{1}^{\prime}, S_{2}^{\prime}$, etc. the diffracted images, called spectra.

In the computing procedure originated by Abbe, formation of the imaged grating in the plane $f^{\prime}$ is investigated in lwo stages: first,

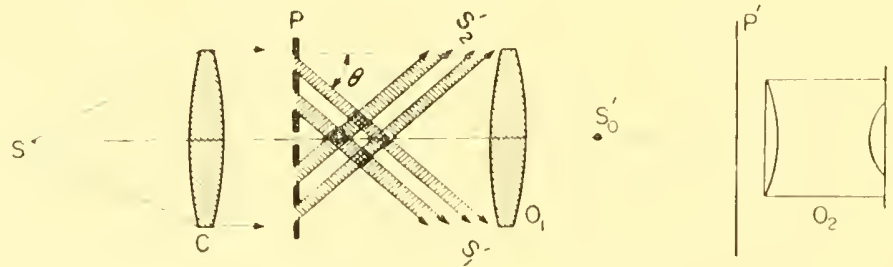

Fig. 1.58. All the spectra $S_{1}^{\prime}, S_{2}^{\prime}, \ldots$ are not transmilled by the objective $O_{1}$.

formation of the images diffracted in the focal plane of the objective $O_{1}$, and next, the change-over from the focal plane to the plane $P^{\prime}$ where the ultimate image is located. The light diffracted by the grating which penetrates in the objective $O_{1}$ is distributed in the images $S_{0}^{\prime}, S_{1}^{\prime}, S_{2}^{\prime}$, etc. These images act as in-phase radiants. When the slits of the grating are very close, spectra $S_{1}^{\prime}, S_{2}^{\prime}$, etc., are far removed from $S_{0}^{\prime}$. To collect the light diffracted in the direction of $\theta$ the objective N. A. must be adequate. If such is not the case, it may occur that all the spectra $S_{1}^{\prime}, S_{2}^{\prime}$, etc., are stopped short by the objective $O_{1}$ (Fig. 1.58). Only 
the light transmitted to $S_{0}^{\prime}$ passes into the objective. The image plane $P$ illuminated solely by the source $S_{0}^{\prime}$ is evenly illuminated. The field of view perceived by the eye from eyepiece $O_{2}$, is uniform and free from the imaged grating. Assuming that the N.A. of objective $O_{1}$ is adequate to let through image $S_{0}^{\prime}$ and spectra $S_{1}^{\prime}$ and $S_{2}^{\prime}$, the plane $P^{\prime}$

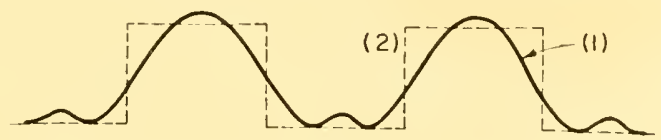

FIG. 1.59. Image of a periodic object when the N.A. of objective $O_{1}$ lets through $S_{0}^{\prime}$ and spectra $S_{1}^{\prime}$ and $S_{2}^{\prime}$.

is then illuminated by three coherent sources: $S_{0}^{\prime}, S_{1}^{\prime}, S_{2}^{\prime}$. Curve 1 in Fig. 1.59 shows the image structure. The pecked curve 2 shows the geometrical image of the grating. Instead of a sharp definition of the bright lines of the grating, the image exhibits shaded-off edges; the image period remains the same as that of the grating. Let us

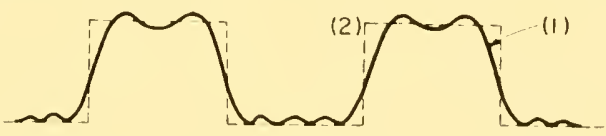

FIG. 1.60. Image of a periodic object when the N.A. of the objective $O_{1}$ lets through $S_{0}^{\prime}$ and two spectra on either side of $S_{0}^{\prime}$.

increase the objective's N.A. to let in two spectra on either side of $S_{0}^{\prime}$. The image then exhibits additional diffraction fringes (Fig. 1.60). As more spectra are let in, the image period does not alter and remains that of the object grating but the outline image gradually tends to

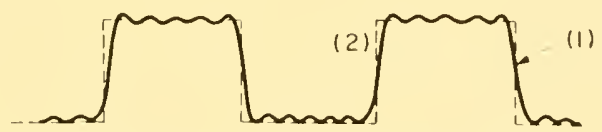

FIG. 1.61. As more spectra penetrate into the objective, the image looks more and more like the object.

a perfect image (Fig. 1.61). The geometrical image is the limit-image achievable were all the spectra involved, that is, with an objective of infinite aperture. If but few spectra are let in, the luminous continuities are not shown in the image and substituted for shaded-off outlines that do improve sharpness of the image. As more spectra 
penetrate into the objective, the image looks more and more like the object.

Let us consider a grating whose slits are very close and let us operate in oblique illumination. The layout may be that shown in Fig. 1.62 where only the direct image $S_{0}^{\prime}$ and the spectrum $S_{2}^{\prime}$ penetrate into the objective. The plane $P^{\prime}$ is illuminated by the two coherent sources $S_{0}^{\prime}$ and $S_{2}^{\prime}$. The imaged grating is a sinusoidal image similar

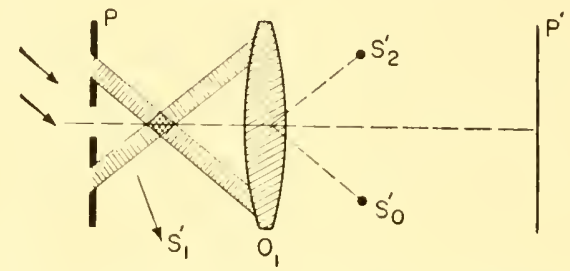

FIG. 1.62. Periodic object in oblique illumination.

to that in Fig. 1.48 (Young's fringes produced by the two sources $S_{1}^{\prime}$ and $S_{2}^{\prime}$ in the plane $P^{\prime}$ ). Therefore, all that is needed to see the imaged grating is that the objective N.A. be such that the image $S_{0}^{\prime}$ plus a spectrum, e.g. $S_{2}^{\prime}$, may penetrate into the objective. Oblique illumination of the grating shows a sinusoidal image the period $p$ of which is given by

$$
p=\frac{\lambda}{2 n \sin u}
$$

The expression shows the resolving power of a Foucault type periodic object in coherent illumination. The numerical value obtained is the same as in incoherent illumination (expression 1.6) although the resolving power is then reached after a steady dwindling of the image contrast. In coherent illumination, the process is different: as long as two images at least penetrate into the microscope's objective the periodic structure is clearly seen. The imaged grating vanishes only when one of the two images $S_{0}^{\prime}$ or $S_{2}^{\prime}$ is arrested by the objective.

\section{PERCEPTION LIMIT AND RESOLVING POWER}

The foregoing results are collected and discussed further in the present section.

If the phenomena are considered in the plane of the specimen, a pin-point detail can be substituted for a small disk of radius 
$1 \cdot 22 \lambda /(2 n \sin u)$. Two pin-point details on black ground, emitting identical luminous fluxes will appear discrete only if the relevant diffraction disks are not too close to one another. Let us first assume that the two pin-point details act as would two incoherent sources on black ground. In Fig. 1.63 the distance between the two diffraction disks

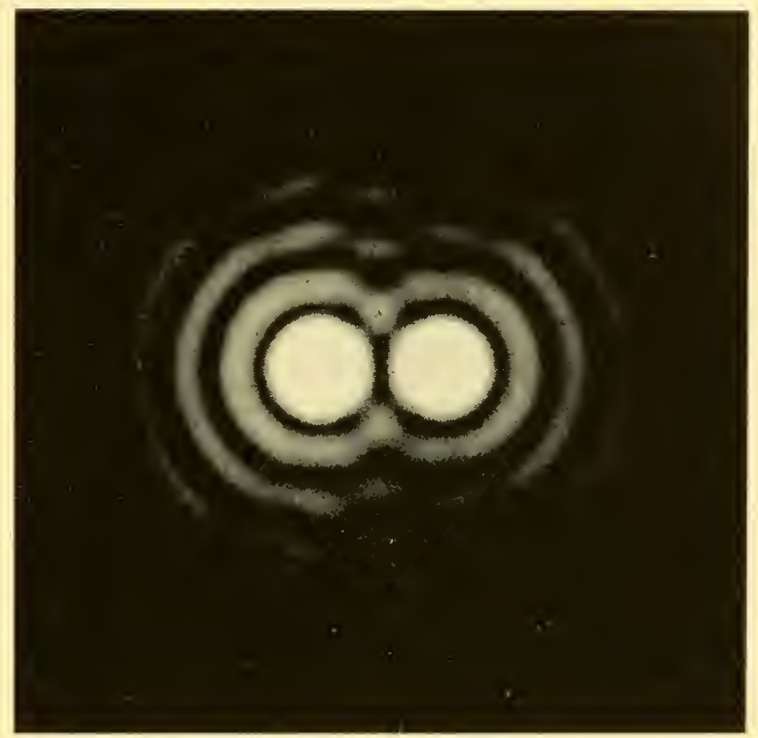

FIG. 1.63. Diffraction patterns the distance of which is greater than their diameter.

amounts to approximately twice their diameter and are readily disjoined. Resolving power of the two disks is usually considered to be reached when the central maximum of one of the diffraction patterns

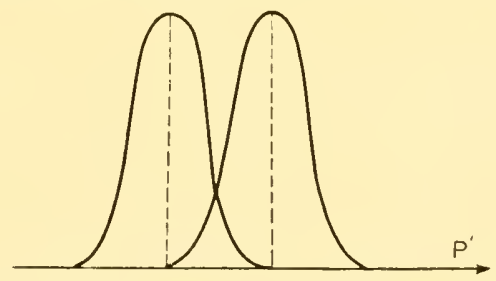

FIG. 1.64. Diffraction curves as resolving power is attained (incoherent illumination).

corresponds to the first zero minimum of the other and conversely, i.e. when the diffraction curves are as shown in Fig. 1.64. Under such conditions, light-intensity, half-way between the two images, 
amounts to $0 \cdot 74$, viz. a loss of 26 per cent. Figure 1.65 shows the two diffraction disks as resolving power is attained. From expression (1.2) the distance between the two luminous points in the specimen plane is given by the expression:

$$
y=\frac{1 \cdot 22 \lambda}{2 n \sin u} .
$$

As the N.A. of the microscope's objective increases the diameter of the diffraction disk shrinks and the closer details are resolvable. Figures 1.66, 1.67 and 1.68 show how an image structure evolves

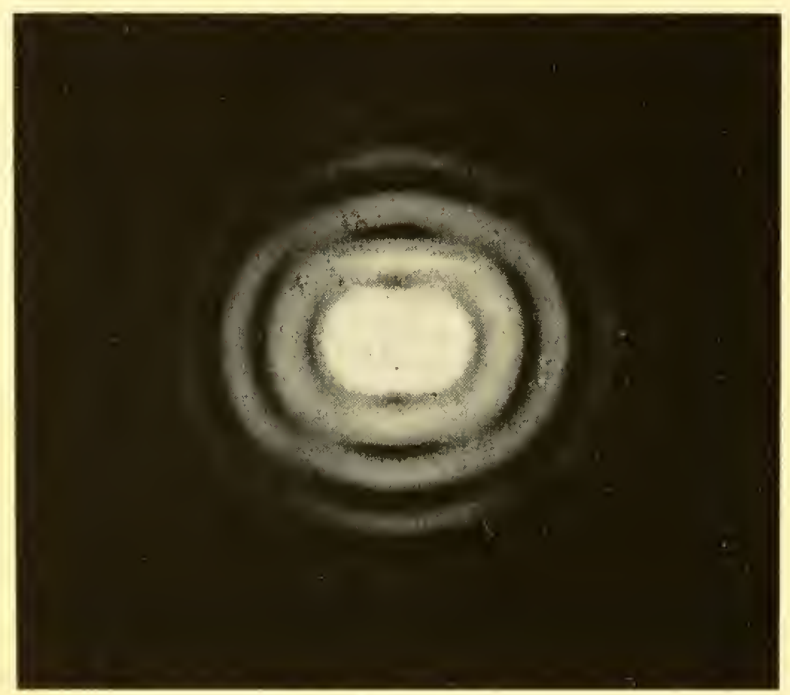

Fig. 1.65. Two diffraction patterns as resolving power is attained (incoherent illumination).

according to the objective's N.A. In Fig. 1.66 the N.A. is inadequate and details cannot be resolved: the image is indifferently defined and surrounded by diffraction fringes. As N.A. increases (Fig. 1.67) the structure outline of the object emerges. As N.A. is increased (Fig. 1.68) the diffraction disks are sufficiently fine to show that the object consists of discrete luminous points. This evinces clearly the significant effects of diffraction on the image structure of small objects. Figures 1.69 and 1.70 are instructive, too: they show the aspect of an object consisting of luminous points on dark ground in coherent and incoherent illumination. The imaged diffraction disks of the luminous points add up in incoherent illumination (Fig. 1.69). Diffraction rings retain their 
Fig. 1.66. Image structure according to the objective's N.A. $n \sin \|=0 \cdot 20$.

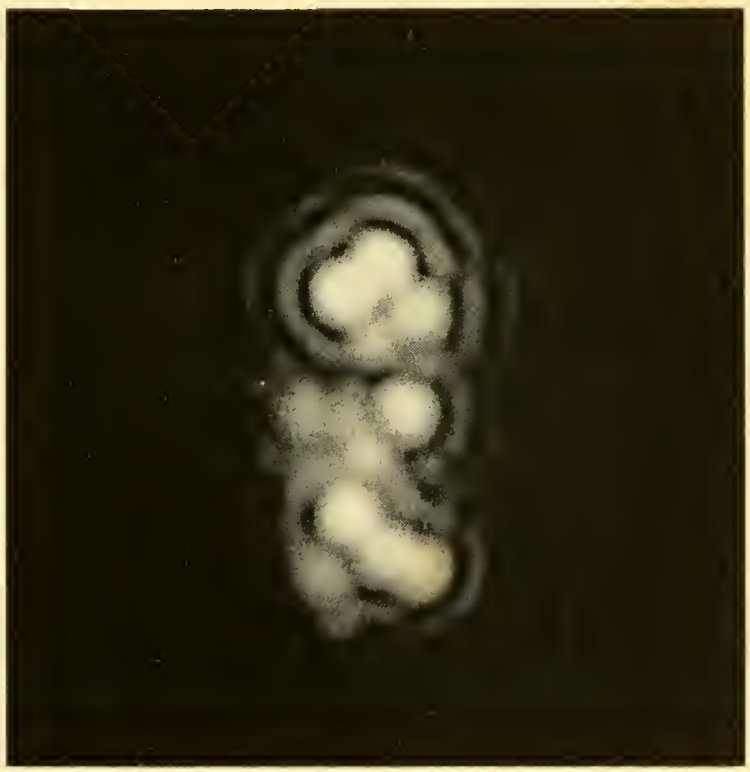

FIG. 1.67. Image structure according to the objective's N.A. $n \sin u=0 \cdot 60$. 
circular shape. Conversely, in coherent illumination (Fig. 1.70) there is some interference between the various diffraction disks. The rings are completely distorted and even the central disks are no longer circular. This would lead one to believe that the actual shapes of

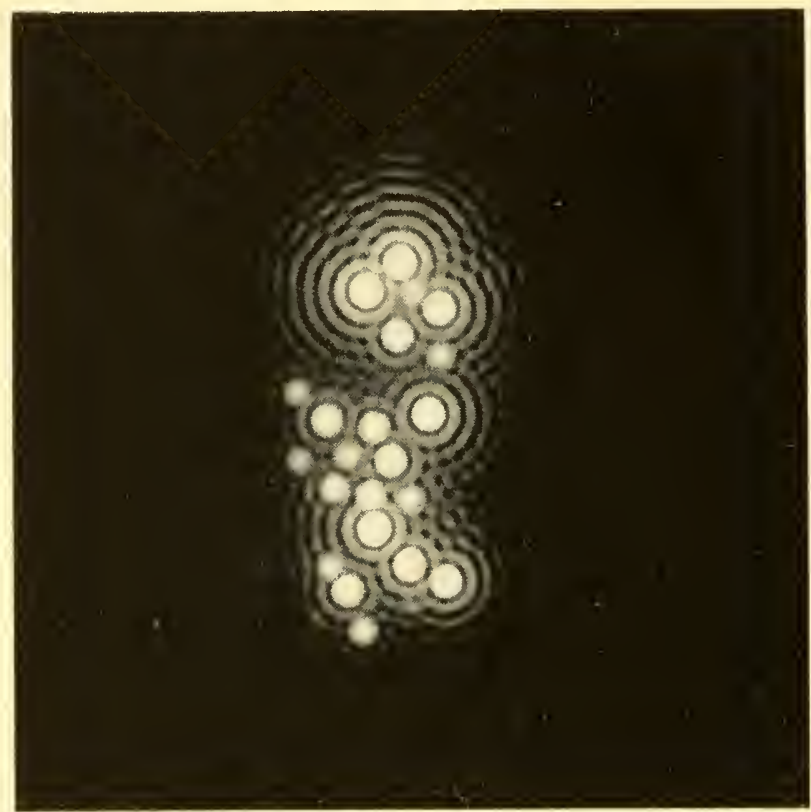

Fig. 1.68. Image structure according to the objective's N.A. $n \sin u=1 \cdot 30$.

luminous objects are emerging but such is not the case, since the errors that may be made when interpreting such images can readily be realized.

Let us now consider the case where two pin-point details act as two coherent sources on black ground. Under the same conditions, resolving power is reached when the distance $y$, from the two points in the specimen plane, is given by:

$$
y=\frac{1.63 \lambda}{2 n \sin u} .
$$

Two pin-point luminous sources on black ground are not as discrete in coherent illumination as in incoherent.

H. H. Hopkins investigated how the separation limit of two pinpoint sources on black ground varies versus the condenser N.A. The 
test is the same as before: resolving power is reached when the drop in intensity between the two images amounts to 26 per cent. If the condenser is almost fully diaphragmed, illumination is coherent and formula (1.11) applicable. The coefficient $K$, by which $\lambda /(2 n \sin u)$ is

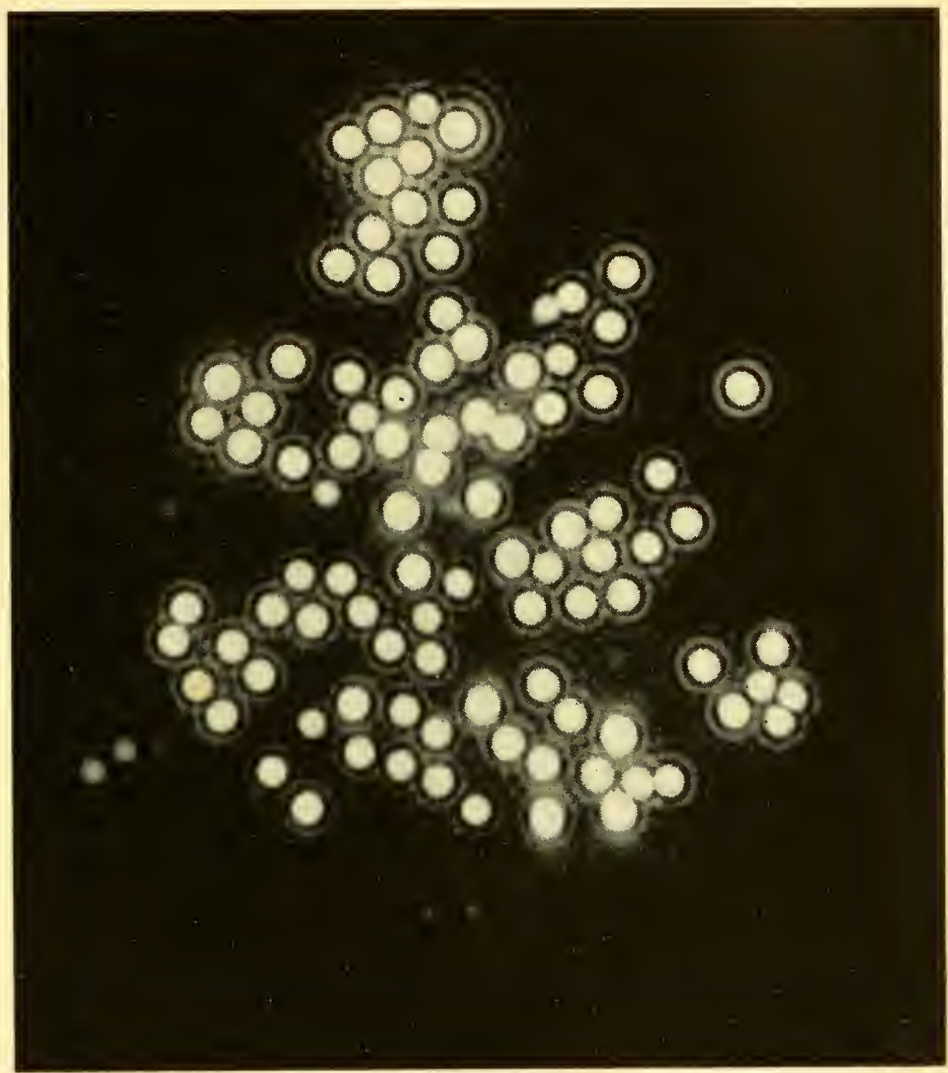

FiG. 1.69. Aspect of an object consisting of luminous points on black ground: incoherent illumination.

to be multiplied in order to have resolving power $y$, is plotted in Fig. 1.71, as ordinate and the ratio $m$ as abscissae

$$
m=\frac{\text { Condenser N.A. }}{\text { Objective N.A. }}
$$

When $m=0$ (coherent illumination), $K=1.63$ (expression 1.11). When $m=1$, i.e. when the N.A. of both condenser and objective is the same (partially coherent illumination), $K=1 \cdot 22$. Separation 
limit is much the same as in incoherent illumination (expression 1.10). The curve shows that resolving power is best when the condenser aperture is 1.5 times higher than the objective's. However, in actual practice, owing to spurious light, this theoretical result is not ap-

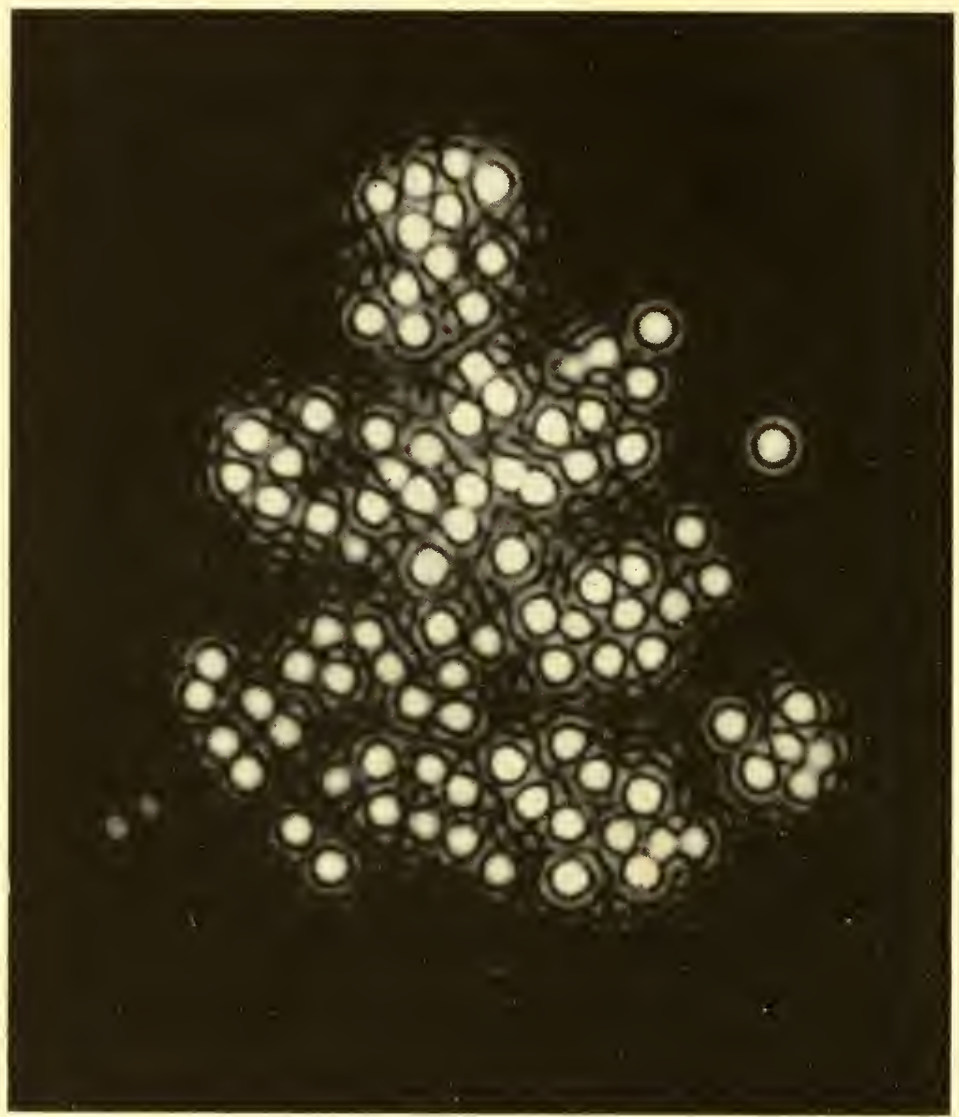

IIG. 1.70. Aspect of an object consisting of luminous points on black ground: coherent illumination.

plicable. Experience shows that when the ratio $m=0.6$ or 0.7 , it can hardly be exceeded without substantially increasing stray light and, concurrently, decreased image contrast.

The phenomenon is the same for a periodic object: resolving power improves as the condenser aperture is increased. In Fig. 1.72, illumination is coherent, the condenser lens is almost fully diaphragmed and phenomena oceur very much as if the light were originated from 
the pin-point source $S_{0}^{\prime}$. The grating $P$, the spectra of which do not penetrate into the objective $O_{1}$, is observed. Only the direct image $S_{0}^{\prime}$ does penetrate into the objective and only a uniform illumination

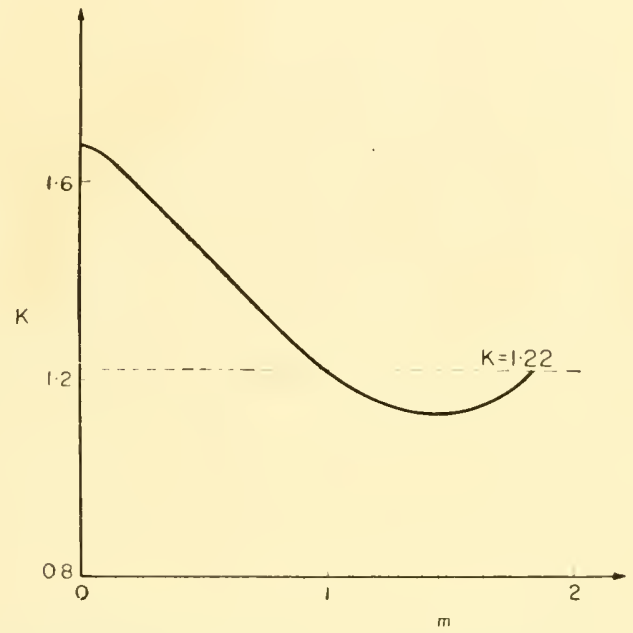

FiG. 1.71. Effect of the condenser aperture on the resolution of two pin-points of equal intensity (after H. H. Hopkins and P. M. Barham).

is visible in the image plane $P^{\prime}$. Let us open the condenser diaphragm: the light is originated from a wide source $S_{1} S_{2}$. The grating $P$ is obliquely illuminated by the edge of source $S_{2}$ and the arrangement

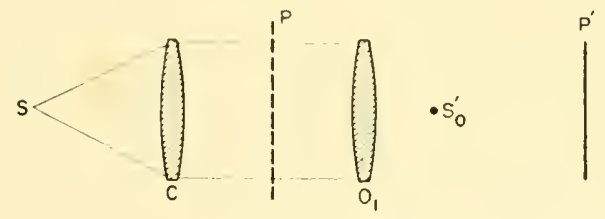

(a)

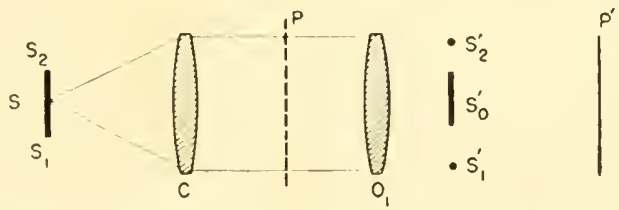

(b)

FIG. 1.72. Effect of the condenser aperture on the resolution of a periodic object.

is very similar to that in Fig. 1.62. The direct image $S_{0}^{\prime}$ spreads out and its lower portion in Fig. 1.72(b) matches the upper portion $S_{2}$ of the source $S_{1} S_{2}$. 
Assuming the edge $S_{2}$ to be at such a distance from the axis that only the very edge $S_{2}^{\prime}$ of the spectrum $S_{2}^{\prime}$ penetrates into the objective $O_{1}$. The lower edge of $S_{0}^{\prime}$ (lower edge of the spectrum $S_{2}^{\prime}$ ) conveys vibrations to the image plane $P^{\prime}$ which may bring about

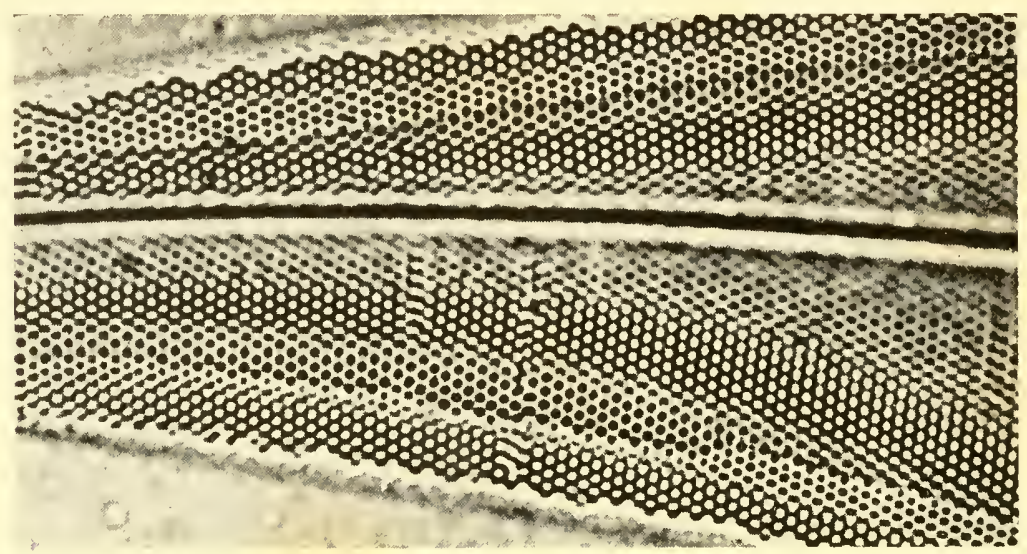

(a)

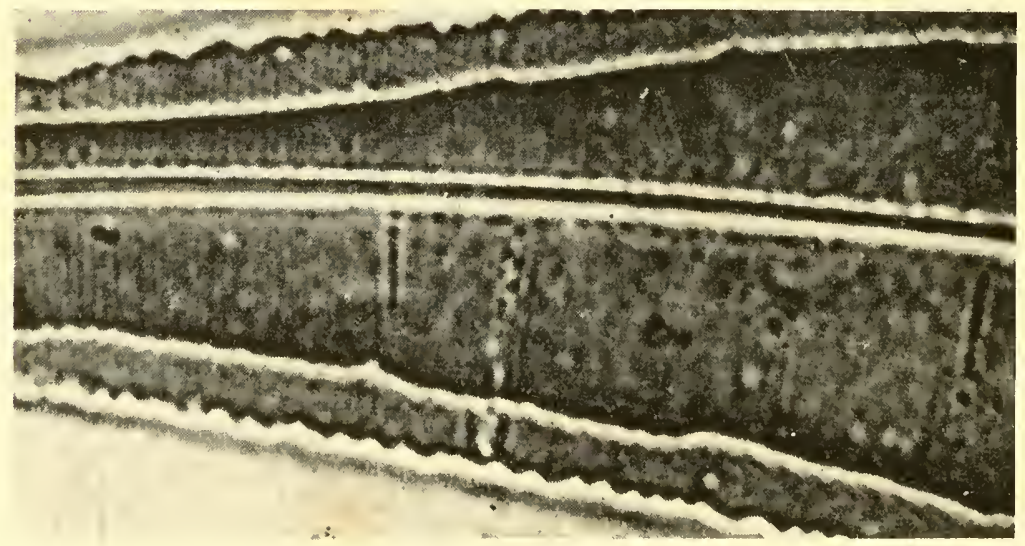

(b)

FIG. 1.73. Plenrosigma angulatmm; plemosigna angulatmm nol resolved.

interference; likewise, the upper edge of $S_{1}^{\prime}$ and $S_{1}^{\prime}$ (upper edge of the spectrum $S_{1}^{\prime}$ ). The plane $P^{\prime}$ is illuminated by several sources which interfere and an image of the grating is perceived.

When the condenser diaphragm is almost fully diaphragmed, neither the spectrum $S_{1}^{\prime}$ nor $S_{2}^{\prime}$ can penetrate into the objective. As the diaphragm aperture increases the spectra $S_{1}^{\prime}$ and $S_{2}^{\prime}$ spread out and a time comes when a small portion thereof penetrates into the objective, whereupon an image of the grating appears. Let us now 
consider a diatom: pleurosigma angulatum (Fig. 1.73(a)). The microscope eyepiece is removed and the objective focal plane observed by means of a focusing telescope. With an objective of N.A. =0.85, and the condenser almost fully diaphragmed, only the direct image $S_{0}^{\prime}$ is visible (Fig. 1.74(a)). Let us put back the eyepiece and observe the imaged diatom: the aspect is that of Fig. 1.73(b): the diatom is

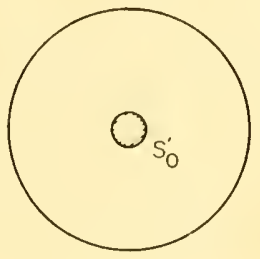

(a)

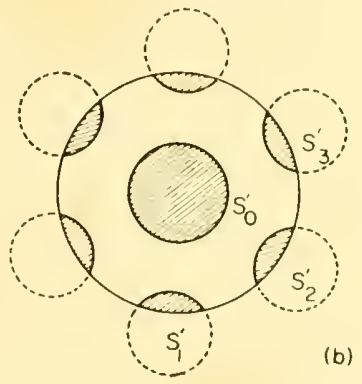

Fig. 1.74. Diffracted light in the focal plane of a microscope objective (pleurosigma angulatum).

not resolved. Let us consider again the objective's focal-plane and open the condenser diaphragm. The spectra outside the field spread out and a time comes when the spectra edges penetrate into the objective (Fig. 1.74(b)) and the imaged diatom is resolved.

Under normal working conditions, perception of discrete objects of high contrast and that are small in relation to the diffraction disk of the objective varies but slightly with condenser aperture: illumination is nearly always coherent. However, when the condenser is diaphragmed too much, images do not exhibit an attractive appearance. Increasing the condenser aperture improves image sharpness and subdues diffraction fringes. Naturally while the condenser N.A. affects but little the perception of minute discrete objects it does take effect on the separation limit of closely adjacent objects. Images are finer and diffraction fringes fade as the aperture of the condenser increases, the more utmost definition is improved (Fig. 1.71).

The numerical data mentioned in the foregoing paragraphs are tabulated below. The data included in chart 1.1 apply to a perfect instrument and should be considered as the very best obtainable. Such results may be approximated by using, in monochromatic light, a microscope with stray light excluded to the utmost and whose objectives are well corrected from spherical aberration. Chart 1.2 shows the chromatism effects on perception limit of a small dark disk 
on white ground. The effects are not marked with an apochromatic objective but, conversely, are significant with an achromatic one.

CHART 1.1

\begin{tabular}{|c|c|c|c|}
\hline & $\begin{array}{l}\text { Coherent illumina- } \\
\text { tion, very small con- } \\
\text { denser aperture }\end{array}$ & $\begin{array}{c}\text { Partially colterent } \\
\text { illmmination, con- } \\
\text { denser aperture the } \\
\text { same as that of } \\
\text { objective }\end{array}$ & $\begin{array}{l}\text { Incoheremt illumina- } \\
\text { rion, (fluorescence) }\end{array}$ \\
\hline $\begin{array}{l}\text { Separation limit of two } \\
\text { bright points on black } \\
\text { ground }\end{array}$ & $y=\frac{1.63 \lambda}{2 n \sin u}$ & $y=\frac{1 \cdot 22 \lambda}{2 n \sin u}$ & $y=\frac{1 \cdot 22 \lambda}{2 u \sin u}$ \\
\hline $\begin{array}{l}\text { Perception limit of small } \\
\text { black disk on white } \\
\text { ground }\end{array}$ & $r=\frac{0.08 \lambda}{2 n \sin u}$ & $r=\frac{0 \cdot 08 \lambda}{2 u \sin u}$ & $r=\frac{0.13 \lambda}{2 n \sin u}$ \\
\hline
\end{tabular}

Perception limit of small white disk on black ground

Perception limit of thin black line on white ground

Perception limit of thin streak of light on black ground

Separation limit of a periodic object (Foucault's test)

$y$ - distance between two points

$r$ disk radius

Pereeption of a small white disk on black ground depends solely on the luminotis flux emitted

$$
\varepsilon=\frac{0.01 \lambda}{2 u \sin u} \mid \begin{array}{c|r}
0.01 \lambda \\
2 u \sin u
\end{array} \quad \varepsilon-\frac{0.02 \lambda}{2 n \sin u}
$$

Perception of thin streak of light on black ground depends solely on the luminous flux emitted

$\varepsilon=$ width of thin line

$p$ - distance between two bright or two opaque lines.

CHART 1.2. CIIROMATIC IFFECIS ON THE IMAGF CONTRASI OF A SMAIL, BIACK DISK ON WIITE GROUNI)

(coherent illumination for cach wave-length)

\begin{tabular}{c|c|c|c|c} 
& Perfect objective & Apochromatic & Fluorite & Achromatic \\
\hline Contrast values & 0.33 & 0.31 & 0.27 & 0.18
\end{tabular}




\section{STRAY LIGHT}

When a light-beam passes through a microscope, a portion of it is reflected on every surface and reverts in the source direction. If this luminous flux is no longer reflected, it can be considered as not taking part in image formation. But if this beam is reflected anew, a portion of it is reverted to the image, forming a halo of stray light. Figure 1.75 shows diagrammatically the luminous paths just described.

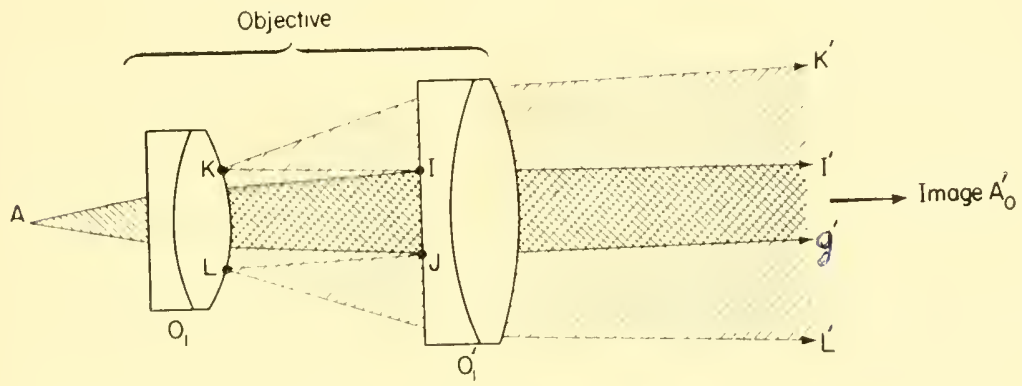

FIG. 1.75. Scattered light reflected by the lenses of an objeclive.

Let us now consider a narrow pencil of rays originated from the luminous source $A$. After passing through the objective's element $O_{1}$ it is reflected at $I J$ on to the element $O_{1}^{\prime}$, next at $K L$ on to the element $O_{1}$ and returns to the image $A_{0}^{\prime}$. The light beam $I J I^{\prime} G^{\prime}$ shares in the formation of the actual image $A_{0}^{\prime}$, but the beam $K L K^{\prime} L^{\prime}$ gives rise to a stray-light halo in the plane of the image $A_{0}^{\prime}$. This halo is not very pronounced. Yet scattered light reflected on the curved sides of elements $O_{1}$ and $O_{1}^{\prime}$ have to be considered, the result being that reflection losses become quite heavy when the instrument has many surfaces. Such losses impair image illumination and produce stray light. Intensity of the stray light may attain and even exceed 5 to 7 per cent of the intensity of the normally illuminated field. Low-contrast, imaged objects may vanish. Therefore reducing to a minimum the stray light reflected between the lenses of the instrument is essential. The light reflected by a glass surface can be reduced by coating it with a thin film of suitable thickness (Fig. 1.76). Reflected light is originated by the interference of rays such as $a$ and $b$ : it is least at wavelength $\lambda_{0}$ if the product ne equates $\lambda_{0} / 4$. Wave-length $\lambda_{0}$ from the minimum is selected, the wave-length for which the receiver exhibits utmost sensitivity. For instance, for the eye, $\lambda_{0}$ is selected close to 
$0.55 \mu$. The amount of reflected light is shown by the expression:

$$
R=\frac{\text { Reflected luminous flux }}{\text { Incident luminous flux }} .
$$

$R$ is the reflectance of the surface. If the glass surface is coated its reflectance is least when the wave-length $\lambda_{0}=0.55 \mu$ and highest when at the blue and red ends of the spectrum, the surface

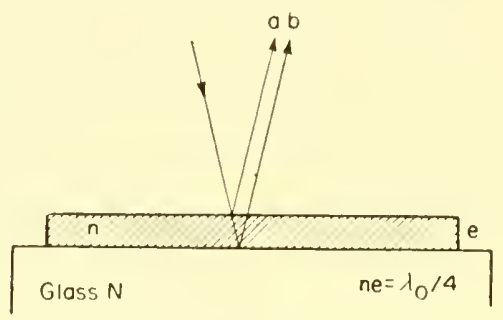

FIG. 1.76. Glass surface coated by a thin film.

then being purplish red if observed by reflection. Let us consider flint-glass refraction index $N=1.62$. Uncoated, its surface has reflectance $R=0.056$ at normal incidence. Coated with a thin film of thickness ne $=\lambda / 4$ when $\lambda_{0}=0.55 \mu$ the reflectance of the glass optical plate is 0.01 when $\lambda_{0}=0.55 \mu$. A still lower reflectance in relation to the wave-length can be obtained by the use of achromatic thin film (Turner). Instead of one, two thin films are now deposited on the glass surface (Fig. 1.77). The first thin film has an optical

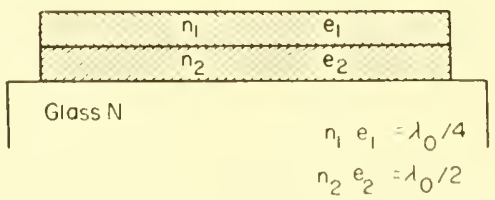

FIG. 1.77. Glass surface coated by two thin films.

thickness $n_{1} e_{1}=\lambda / 4$, that of the second one $n_{2} e_{2} \cdots \lambda / 2$ (between the first and the glass). Figure 1.78 shows the reflectance distribution of $N$-index glass in relation when $N=1.62, n_{1}=1.4$ and $n_{2}=2.0$. Curve 1 shows the $R$ changes when the glass surface is coated with one thin film. Curve 2 shows the variations of $R$ when there are two achromatic thin films on the glass. At wave-length $\lambda_{0}=0.55 \mu$ 
the same reflectance is obtained in both cases but the smallest divergence from this wave-length causes the reflectance to differ widely. In this way, stray light in microscopes was much curtailed. Intensity

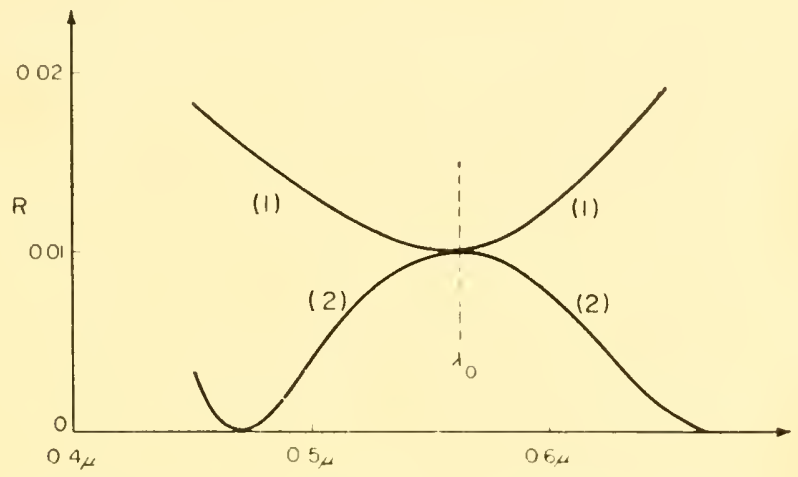

FIG. 1.78. Reflectance variations of a glass: (1) glass surface coated with one thin film; (2) glass surface coated with two achromatic thin films.

of the stray light can be reduced almost tenfold and concurrently improves the instrument considerably.

\section{REDUCING THE GRAIN OF PHOTOGRAPHIC IMAGES}

In some applications, the objectionable grain of photographic plate is evidenced. A typical example is that of a microscope autoradiograph. The granular structure of a photographic image can be reduced by the following process (Fig. 1.79). A fine radiant $S$ in the focus of

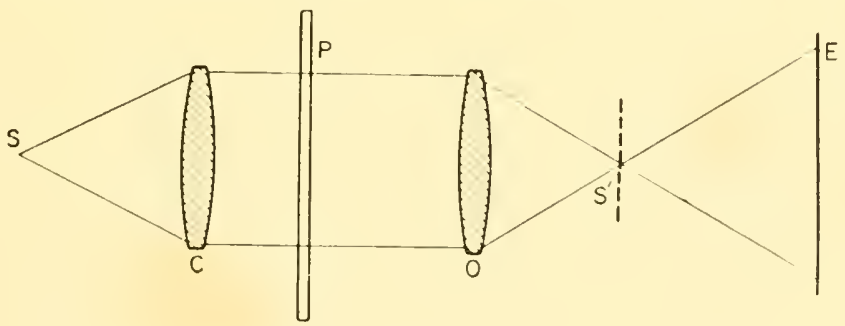

FIG. 1.79. Optical filtering of a photographic image.

the objective $C$ illuminates, with a parallel beam, the photographic plate $P$ requiring improvement. The objective $O$ images the plate $P$ on to $E$. The imaged source $S$ is at $S^{\prime}$. This image is a diffraction 
phenomenon typical of amplitude and phase distribution in the photographic plate. Assuming the phase variations to be negligible and considering amplitude changes only, the image shown in Plate $P$ is

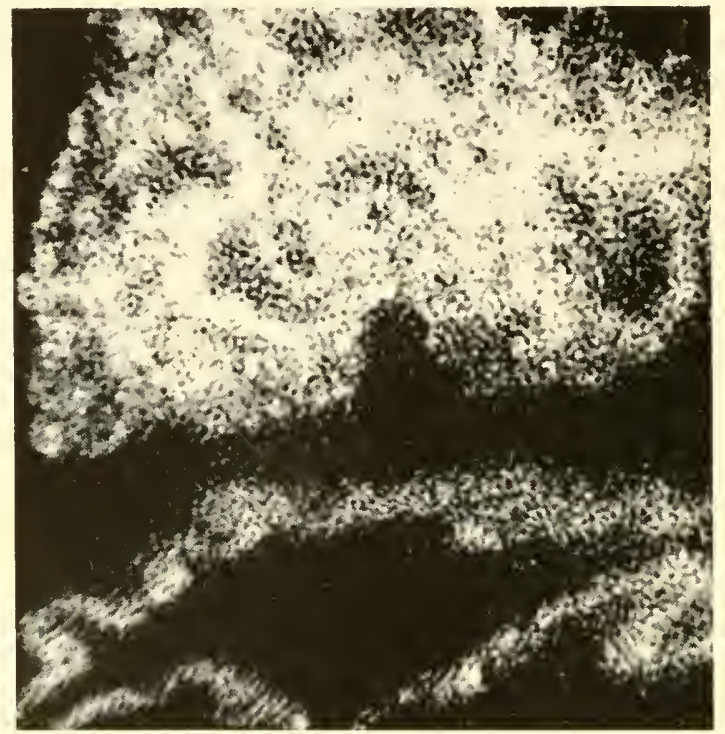

FIG. 1.80. Hepato-pancreas of a snail (helix) (autoradiography).

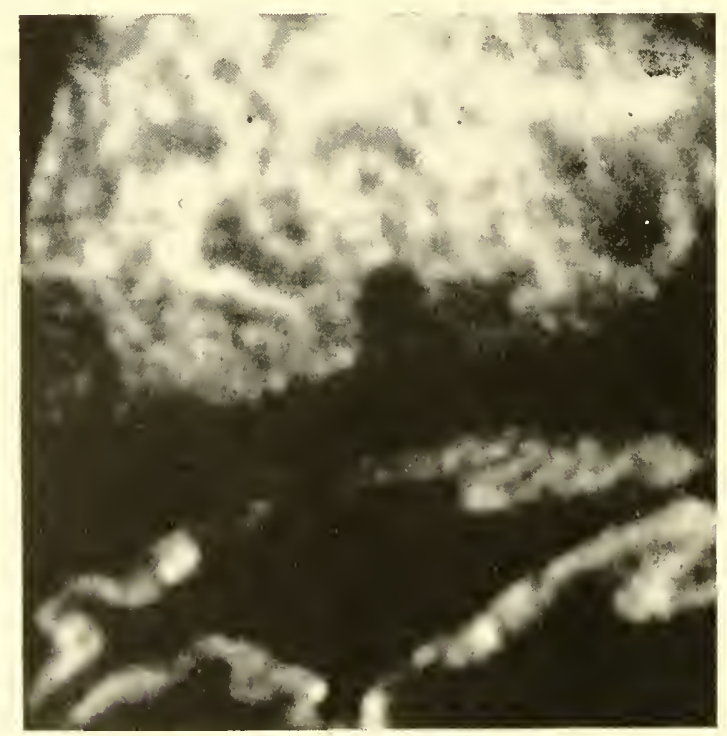

FIG. 1.81. Hepato-pancreas of a snail (helix) after filtering. 
featured by degrees in blackness, i.e. changes in contrast. In this image the grain is evinced as, for instance, in Fig. 1.80. The contrast changes in plate $P$ are details needing investigation. Assuming, as is the case in Fig. 1.80, that these details are much wider than the grains to be eliminated: since the grains are minute and virtually of the same diameter, they exhibit at $S^{\prime}$ an extensively spread diffraction phenomenon. Conversely, the photographic details to be investigated at $S^{\prime}$ are much narrower than the grain's. Setting in the plane $S^{\prime}$ a screen pierced with a hole of suitable diameter (determined by experience) the major part of the grain-originated diffraction phenomenon can be occluded. The narrow diffraction phenomena originated by the photographic details can pass through and a new plate is ultimately obtained at $E$, where grain effects are substantially reduced. The screen, located at $S^{\prime}$ and pierced with a hole, "filters" the photographic plate $P$. Figure 1.81 shows the photograph in Fig. 1.80 after such "filtering". The experiment requires much care in order to prevent interpretation errors. 


\section{CHAPTER II \\ Phase Contrast Microscopy}

\section{TRANSPARENT OBJECTS}

A TRANSPARENT object is an object that can only be distinguished in the medium encompassing it by the variations of $\mathrm{its}$ refractive index or its thickness. No variation in light-intensity will detect its presence, the field of view remaining uniform. Such objects, frequently called "dephasing", obviously escape normal observation although their role in microscopy is a large one.

Transparent objects are many in biology and microscopists have to stain them in order to render them visible. This method is particularly detrimental when living objects are observed and this fact gives much prominence to phase- and interference-contrast methods as they enable one to study living tissues under conditions just as good as when studying tissues that died after staining.

Transparent objects are met with in many other branches of science besides biology. For instance, inequalities on metallic surfaces having a uniform reflection act as transparent objects and can be observed by either phase- or interference-contrast methods. Structural unevenness in crystals, index variations in an inhomogeneous liquid, deformations of a liquid surface and so forth are transparent objects. Even when not unduly small, transparent objects are not perceivable in ordinary microscopes because they lack in contrast when compared to the remainder of the field. Both contrast and interference microscopes render such objects visible.

\section{PRINCIPIE OF THE PHASE-CONTRAST METHOI)}

The phase-contrast method was invented by the Dutch physicist Zernike who was awarded the Nobel prize in 1953.

Originally used by its inventor for inspecting telescope mirrors, the phase contrast technique was applied to microscopy shortly after- 
wards. Zernike's initial work was published before the last war but effective development of phase-contrast microscopy was not under way before 1946. Complete investigation of phase contrast requires some mathematical data which are not indispensable, however, if the study is restricted to basic phenomena. $P$ is a transparent object observed in a compound microscope (Fig. 2.1). The filament of the

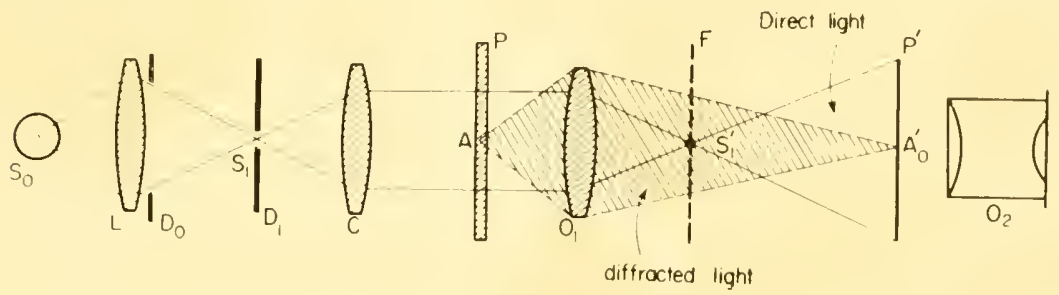

FiG. 2.1. Diagram of the ray paths through a microscope.

source $S_{0}$ is focused on to the iris diaphragm $D_{1}$ of the condenser through the lens $L$. The diaphragm $D_{1}$ is in the focal plane of the condenser's optical system $C$. Under such conditions, were the source but a pin-point, a beam of parallel rays would pass through the specimen $P$. In fact, the source is always spread over a certain area: each of its points originate a pencil of parallel rays so that an infinity of such pencils pass through the specimen at various angles. Against the lens $L$ is the iris diaphragm $D_{0}$ whose distance from the condenser is such that $C$ actually images $D_{0}$ on to the specimen. The specimen $P$ is observed by the microscope proper, consisting of the objective $O_{1}$ and the eyepiece $O_{2}$. The afore-mentioned Köhler illuminating system is used.

Let us consider, for instance, a small transparent bacterium $A$, assuming the lamp $S_{0}$ to be a source of monochromatic light. The bacterium is invisible, not on account of its size, but merely because its contrast in relation to the remainder of the field is too low. Owing to the incident light, the bacterium diffracts a small amount of light in a cone that widens out as the bacterium is smaller. In Fig. 2.1 the hatched area shows the portion of diffracted light that passes into the microscope.

Let $D_{1}$ be substantially diaphragmed down, thus using only but a small portion of the filament imaged at $S_{1}^{\prime}$. In this plane, the beam of direct light covers but a very narrow area whereas the beam of diffracted light overspreads amply. This point is essential and will 
be applied later. Let us now investigate the area wherein the small bacterium $A$ lies. Let $n$ be the refraction index of the latter and $n^{\prime}$ the index of the liquid it is immersed in (Fig. 2.2). If the index is slightly larger than $n^{\prime}$, the light passing through the bacterium (ray 1) lags in relation to the light traversing the liquid close to the bacterium

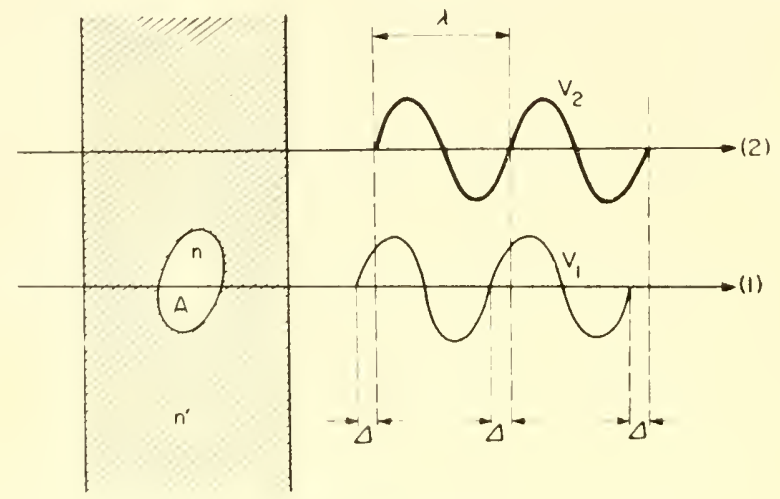

FIG. 2.2. Optical path difference produced by the object $A$.

(ray 2). If $e$ is the bacterium thickness, the difference in the optical path is $A=\left(n-n^{\prime}\right) e$. The sinusoids $V_{1}$ and $V_{2}$ denote the luminous vibrations propagating along rays 1 and 2 , respectively. Since $n>n^{\prime}$ $V_{1}$ lags in relation to $V_{2}$. Such lag is denoted by the short distance 1 in Fig. 2.2. In the following, it is assumed that the lag is small in

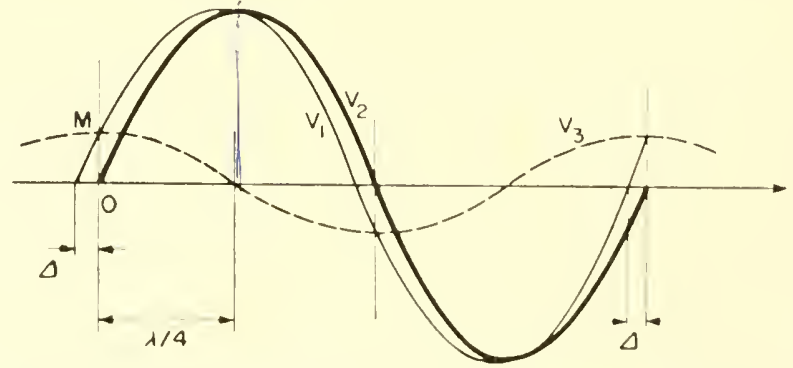

FIG. 2.3. Graphical relations among the $V_{1}, V_{3}$ and $V_{3}$ wilves such that $V_{1}=V_{2}+V_{3}$.

relation to wave-length $\lambda$. Figure 2.3 shows both sinusoids $V_{1}$ and $V_{2}$, side by side and only out of phase by the short distance 1 , as shown in Fig. 2.2. It is well known that a sinusoid. i.c. $V_{1}$ or $V_{2}$, can be shown by the equation: $y=a \sin 2 \pi x / \lambda$, where $y$ is the ordinate of the curve corresponding to abscissa $x$, $a$ the amplitude (highest 
value of the ordinate). Both sinusoids $V_{1}$ and $V_{2}$ are identical as the bacterium is invisible throughout the field, viz., the light-intensity is constant over the whole field. Now, intensity being proportional to the square $a^{2}$ of amplitude $a$, all vibrations passing through the specimen have the same amplitude and, more particularly, the vibrations $V_{1}$ and $V_{2}$ which, henee, are identical.

These two vibrations, although but slightly shifted in relation to one another, do exhibit either the path difference $I$ or the phase difference $p=2 \pi \Delta 1 / \lambda$. Assuming the $1 / \lambda$ ratio and, therefore, $q$ to be small, the square $p^{2}$ of the phase difference $q$ can be disregarded. Let us consider the sinusoid $V_{1}$ denoting the vibration traversing the bacterium. It can be assumed that this sinusoid is the sum of 2 sinusoids:

(a) Sinusoid $V_{2}$,

(b) Sinusoid $V_{3}$ (shifted) by $\lambda / 4(\pi / 2$ in phase difference) in relation to the former and of low amplitude $O M$. A glance at Fig 2.3 reveals that the summed ordinates of these two curves $\left(V_{2}\right.$ and $\left.V_{3}\right)$ do evince sinusoid $V_{1}$ at once. The equation: $y=a \sin 2 \pi x / \lambda$ readily yields the amplitude $O M$ : all that is required is to substitute $x$ for 1 since $x / \lambda$, i.e. $\Delta / \lambda$, being small, $\sin 2 \pi 1 / \lambda$ can be substituted for $2 \pi 1 / \lambda$. Under these conditions, then:

$$
O M=a \frac{2 \pi / 1}{\lambda}=a_{i}
$$

Let us write $a=1$ and, hence, the amplitude $O M$ of the vibration $V_{3}$ is denoted merely by $\%$. Let us now give a physical meaning to vibrations $V_{1}, V_{2}$ and $V_{3}$ by going into Figs. 2.1 and 2.3. The sinusoid $V_{3}$ matches the bacterium-diffracted vibrations; its amplitude $O M$ is proportional to $q$ and is the lower as the $n-n^{\prime}$ difference is smaller. If $n=n^{\prime}$ the phase difference between $V_{1}$ and $V_{2}$ i.e. the phase difference between rays 1 and 2, Fig. 2.2, is zero. The vibration $V_{3}$ vanishes and there is no diffracted light any longer. As to the vibration $V_{2}$, it denotes the direct light passing at $S_{1}^{\prime}$ in Fig 2.1, which is spread over the image $P^{\prime}$.

To sum up, the vibration $V_{1}$, passing through the bacterium can be divided in two: (a) the vibration $V_{2}$, which is the same as in the remainder of the field where there is no bacterium; (b) the vibration $V_{3}$, diffracted by the bacterium and the amplitude of which, proportional to $r$, is all the smaller as $n$ is closer to $n^{\prime}$.

The direct and the diffracted vibrations, $V_{2}$ and $V_{3}$, respectively, are shifted by $\lambda / 4$ (phase difference: $\pi / 2$ ) regardless of the $\gamma$ value, 
provided " remains small. Let us now consider the image $P^{\prime}$ of the specimen $P$ (Fig. 2.4). The bacterium image $A_{0}^{\prime}$ is the outcome of the compounded vibrations $V_{2}$ and $V_{3}$, phase shifred by $\pi / 2$ and reaching $A_{0}^{\prime}$. Classical rules of vibration compounding show that when two vibrations

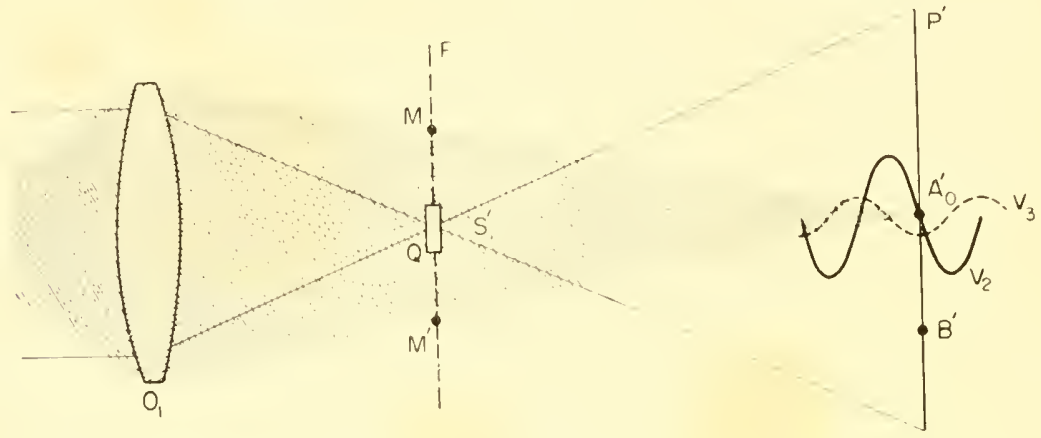

Fig. 2.4. In the image $A_{0}^{\prime}$, sinusoid $V_{3}$ (diffracted light) is shifted by $\lambda / 4$ in relation to the sinusoid $V_{2}$ (direct light).

are out of phase by $\pi / 2$ (lag of $\lambda / 4$ ) the squared amplitude of the resulting vibration, i.e. the light-intensity in the bacterium image $A_{0}^{\prime}$, is given by summing the squared amplitudes of the constituent vibrations $V_{2}$ and $V_{3}$.

Since $\eta^{2}$ is disregarded, the intensity $I_{1}$ in the image $A_{0}^{\prime}$ is given by:

$$
I_{1}=1+r^{2} \simeq 1
$$

In random point $P^{\prime}$, where there are only direct vibrations whose amplitude equates unity, the intensity is, then,

$$
I_{2}=1 \text {. }
$$

Light intensity is the same at all points of the image $P^{\prime}$ : the bacterium is invisible.

Let us assume that, by means of a suitable experiment described later, it were possible to dephase again the sinusoid $V_{3}$ by $\pi / 2$. Let us now observe the image $P^{\prime}$ under such conditions. The vibrations $V_{2}$ and $V_{3}$, reaching the imaged bacterium, are shaped either as shown in Fig. 2.5, (a) and (b), respectively. The diffracted vibrations $V_{3}$ lead in relation to direet vibrations $V_{2}$ or, which amounts to the same thing, the direct vibrations $V_{2}$ are lagging in relation to $V_{3}$. In the case of Fig. 2.5(b), the converse occurs. Both vibrations, $V_{2}$ and $V_{3}$, are in phase in Fig. 2.5(a) and, then, the resulting amplitude equates 
the 1+q summation of the constituent-vibration amplitudes: The light intensity $I_{1}$ in the imaged bacterium $A_{0}^{\prime}$ then becomes:

$$
I_{1}=(1+q)^{2} \simeq 1+2 p
$$

since $q^{2}$ is disregarded. Next to the bacterium, the intensity $I_{1}$ remains equal to 1 . The intensity $I_{1}$ in the imaged bacterium differs from the intensity $I_{2}$ in the remainder of the field: the bacterium becomes visible.

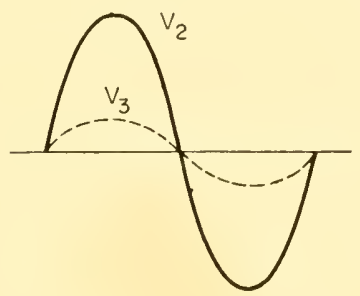

(a)

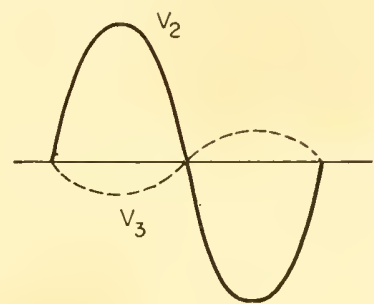

(b)

FIG. 2.5. By introducing the phase plate, sinusoid $V_{2}$ and $V_{3}$ are in phase (a) or in opposition (b).

In Fig. 2.5(b), the vibrations $V_{2}$ and $V_{3}$ are in opposition and are subrtacted from one another. The resulting amplitude in the imaged bacterium is $1-q$ and the intensity:

$$
I_{1}=(1-q)^{2} \simeq 1-2 q .
$$

$I_{1}$ differs from $I_{2}$ : the bacterium is still visible.

In the first case (Fig. 2.5(a)), $I_{1}$ is larger than $I_{2}$. The bacterium outshines the remainder of the field. In the second case (Fig. 2.5(b)), $I_{1}$ is smaller than $I_{2}$ and the bacterium is not as bright as the remainder of the field. Thus shifting the curve $V_{3}$ by $\pi / 2$ in relation to its initial position, i.e. dephasing the direct vibrations by $\pi / 2$ in relation to the diffracted ones, enables one to convert the small phase differences in the object (index or thickness differences) in light-intensity differences in the image. This is the basic principle of phase-contrast microscopy.

We have just seen now phase-shifting of direct and the diffracted light by $\pi / 2$ allows observation of a specimen consisting of small transparent details. How can the motion of sinusoid $V_{3}$ be achieved? Reference to Figs. 2.1 and 2.4 shows that this problem can be solved in a simple manner. We have seen that the direct illuminating light and the bacterium-diffracted light are severed in the plane $F$ passing 
through the image $S_{1}^{\prime}$. This is where the effect on the direct light can be taken without much alteration of the diffracted light. A small transparent plate is placed at $S_{1}^{\prime}$ so that all the direct light passes through it, the small portion of diffracted light passing through the plate being disregarded. Afier selecting the proper thickness and index for the plate $Q$ (Fig. 2.4), (called phase-plate), the direct vibrations (sinusoid $V_{2}$ ) can be made to lead or lag without altering the diffracted vibrations (sinusoid $V_{3}$ ). Figure 2.3 shows that the phase-plate is to lead or lag the direct vibrations $V_{2}$ by a quarter wave-length in relation to the diffracted vibrations $V_{3}$ : the phase-plate is a quarter-wave-length plate. The optical path of the direct vibrations traversing the phase-plate of thickness $e$ and of index $N$ is denoted by $N e$ while the optical path of the diffracted vibrations passing next to it, e.g. in air, is denoted by $c$. If $\lambda$ is the wave-length of the light used, then:

$$
N e-e=(N-1) e=\lambda / 4 \text {. }
$$

The direct vibrations are lagging and the arrangement is that shown in Fig. 2.5(a): the phase-contrast is called negative phase-contrast. Let us increase the thickness $e$ of the phase-plate so that:

$$
(N-1) e=3 \lambda / 4 .
$$

The lag of the direct vibration is now $3 \pi / 2=2 \pi-\pi / 2$. As a $2 \pi$ lag does not change anything, phenomena occur as if the direct vibration led by $\pi / 2$, as in the arrangement shown in Fig. 2.5(b). Phasecontrast is then positive. Assuming that $n$ is larger than $n^{\prime}$, the bacterium outshines the remainder of the ficld in negative phasecontrast and is less bright in positive phase-contrast. The results are tabulated hereunder.

$n$ index of observed detail (bacterium)

$n^{\prime}=$ index of medium encompassing the detail observed.

Negative phase-contrast (the phase-plate delays direct vibrations)

$n$ n' the detail perecived is brighter thatn the remainder of the field.

" $" \prime$ the detail perecived is less bright than the remainder of the ficld.
Positive phase-contrast (the phatse-plate delays diffracted vibrations)

the detail perecived is less bright than the remainder of the lield.

the detail pereeived is brighter than the remainder of the field. 


\section{CONTRAST AND SENSITIVENESS OF THE METHOD}

Resuming the definition given in $\S 1.8$, let us investigate the bacterium contrast. The intensity $I_{1}$, in the imaged bacterium is $=1+2 q$ and the intensity $I_{2}$, in the remainder of the field, is unity. The bacterium image-conirast is

$$
\gamma=2 \gamma \text {. }
$$

If the index $n$ of the bacterium is not very different from index $n^{\prime}$ of the medium encompassing it, both $q$ and contrast $\gamma$ are small. The contrast $\gamma$, under which the bacterium is observed, can be increased, thus improving observation. Let us assume that the phaseplate $Q$ be absorbent. This change does not alter the vibration $V_{3}$ in any way since the diffracted light it represents passes almost wholly close to the phase-plate. Conversely, the greater the phase-plate absorption the less the amplitude of the sinusoid $V_{2}$ (direct light). Let us denote absorption of the phase-plate by the number $N$ that divides the direct light-intensity passing through it. If $N=1$, the phase-plate is transparent; if $N=10$, it only lets through one-tenth of the direct-light incident intensities and so forth. Then, the bacterium image contrast is given by the expression

$$
\gamma=2 \varphi / N \text {. }
$$

In relation to the transparent phase-plate, image contrast is multiplied by,$N$. For instance, if $N=2500$ path differences of approximately 1 ångström may be observed wiih a contrast amounting to $0 \cdot 1$ being still clearly visible. This shows that the phase-contrast method ensures great sensitivity provided, however, that the optical system be of adequate quality and, more particularly, devoid of stray light. The latter, always present in a microscope, is the reason why phase-plates exceeding the value $N=100$ are seldom used. Table 2.1

TABlE 2.1. IMAGE CONTRAST ACCORDING TO VARIOUS PHASE-PLATE ABSORPTION

\begin{tabular}{|c|c|c|c|c|c|c|c|c|}
\hline \multirow[b]{2}{*}{$n-n^{\prime}$} & \multicolumn{2}{|c|}{$\begin{array}{l}\text { Transparent } \\
\qquad N=1\end{array}$} & \multicolumn{2}{|c|}{$\begin{array}{c}\text { Fivefold } \\
\text { absorption } \\
N=5\end{array}$} & \multicolumn{2}{|c|}{$\begin{array}{c}\text { Tenfold } \\
\text { absorption } \\
N=10\end{array}$} & \multicolumn{2}{|c|}{$\begin{array}{l}\text { Fiftyfold } \\
\text { absorption } \\
N=50\end{array}$} \\
\hline & 0.0005 & 0.001 & 0.0005 & 0.001 & 0.0005 & 0.001 & 0.0005 & 0.001 \\
\hline$e=1 \mu$ & 0.01 & 0.02 & 0.02 & 0.04 & 0.03 & 0.06 & 0.07 & $0 \cdot 14$ \\
\hline$e=5 \mu$ & 0.05 & $0 \cdot 10$ & $0 \cdot 10$ & $0 \cdot 20$ & 0.15 & $0 \cdot 30$ & $0 \cdot 35$ & $0 \cdot 70$ \\
\hline$e=10 u$ & $0 \cdot 10$ & $0 \cdot 20$ & $0 \cdot 20$ & $0 \cdot 40$ & $0 \cdot 30$ & $0 \cdot 60$ & $0 \cdot 70$ & 1 \\
\hline
\end{tabular}
RATES 
shows the image contrast-values according to various rates of absorption of the phase-plate versus the thickness $e$ of the detail observed and of its $n-n^{\prime}$ index difference from the medium encompassing it.

Thus, in a detail 10 microns thick, having an index difference $n-n^{\prime}=0.001$ from the ambient medium, a $0 \cdot 20$ contrast obtains when the phase-plate is transparent. This is not a low contrast and yet such a value does not provide comfortable observation. The same object, seen with a phase-plate dividing the incident intensity by 5 $(N=5)$, increases contrast to $0 \cdot 4$ : a satisfactory level. Utmost contrast, for this object, is obtained by a phase-plate dividing incident intensity by 50 . The foregoing results imply a small $q$. Now, this is not always feasible in microscopy and ascertaining the image-contrast variation versus the phase-shift $\varphi$ set in by the object is useful. Figure 2.6 shows the results evinced by two phase-plates setting - in the normal $\pi / 2$ phase displacement between the diffracted and the direct light. The first is transparent $(N=1)$, the second absorbing $(N=10)$.

With a transparent plate, the contrast peaks when the phase displacement set in by the object is $45^{\circ}$ and cancels out when $\gamma=90^{\circ}$. With an absorbing phase-plate, variations occur much faster: when $N=10$, maximal contrast obtains when the phase-displacement set in by the object approximates $18^{\circ}$ and cancels out when $\gamma=34^{\circ} 7^{\prime}$. The contrast is reversed at $\phi$ values exceeding $90^{\circ}(N=1)$ and $34^{\circ} 7^{\prime}$ $(N=10)$. If $q$ remains small the portions $O A$ and $O B$ of these two curves may be considered as being virtually rectilinear as shown in formulae 2.8 and 2.9. In both these regions the contrast $\gamma$ is proportional to the phase difference $\%$. When a specimen contains some details exhibiting manifold $q$ values, the curves in Fig. 2.6 evidence that all the details cannot be descried at the same time with the same phaseplate. Some are discerned with a high, some with a low contrast. Details conforming to $\gamma=90^{\circ}$ are invisible with a transparent phase-plate.

Richter has shown that a $\pi / 2$-dephasing-plate is not the most efficient onc. The phase-displacement $\psi$ originated by the phase-plate, for an object conforming to phase-difference $r$, should be:

$$
y=90^{\circ}-r / 2
$$

with absorption $N$ :

$$
\mathrm{I} / N=4 \cos ^{2} y^{\prime}
$$


If $q$ is small, $w$ is virtually $=90^{\circ}$ and the most efficient phaseplate is a quarter-wave one. Figure 2.3 shows the relevant result. Phase-plates exhibiting variable characteristics aı, hence, valuable for

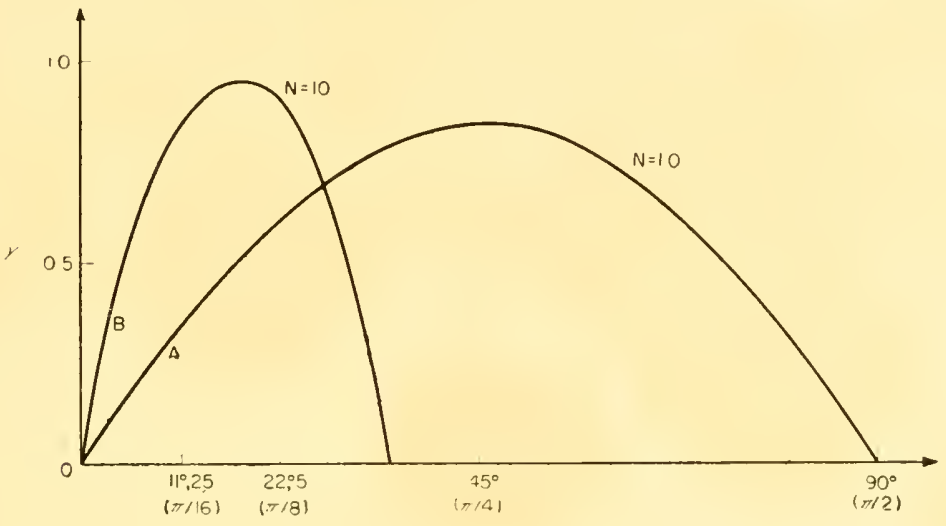

FIG. 2.6. Contrast image $\gamma$ in relation to the phase difference $\gamma$ produced by the object.

they allow one to go fully into the specimen details. However, microscopes suited to phase-plates of this type are usually elaborate instruments requiring much skill.

\section{IMAGE STRUCTURE IN PHASE-CONIRAST}

Referring to Fig. 2.7, the diagrammatic object shown is the dephasing object. The latter may be, e.g. a parallel-sided glass plate with a rectangular band $A$. Positive phase-contrast observation shows the area $A$ in black against a white ground but the image structure

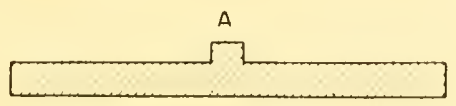

FIG. 2.7. Diagrammatic dephasing object.

varies as the width of the object $A$. Assuming the latter to be minute, the light it diffracts overspreads along the plane $F$ (Fig. 2.4); the small portion of diffracted light passing through phase-plate $Q$ can be disregarded. Under such conditions, the image structure is that shown in Fig. 2.8(a). The imaged object as seen is correct: there is a small dark band on a light ground the outlines of which are rounded off by diffraction. As the band-width $A$ increases, overspreading of the 
light diffracted along the plane $F$ recedes (Fig. 2.4). The portion of diffracted light passing through the phase-plate can no longer be disregarded as it brings about the perturbations shown in Fig. 2.8(b). The image still conforms to the object and a dark band on light ground is discernible but the image is edged by the two clearly visible bright fringes $m$ and $m^{\prime}$. The halo that always surrounds phase contrast images is due to these fringes. Naturally, this halo is also present

(a)

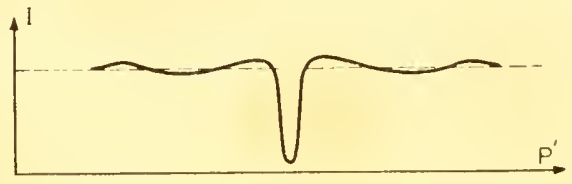

(b)

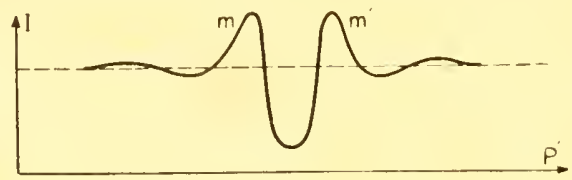

(c)

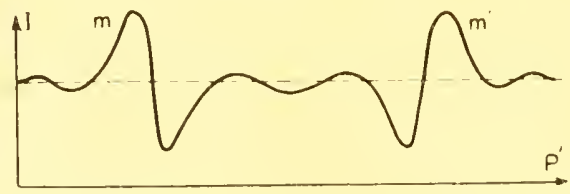

FIG. 2.8. Image structure in phase contrast method (after H. Wolter).

in Fig. 2.8(a) but much more extensive and not so visible. If the width of the band $A$ continues to increase, a time comes when the width of the diffraction phenomenon it originates along the plane $F$ (Fig. 2.4) is approximately the same as that of phase-plate. Now the image is completely perturbed, as shown in Fig. 2.8(c.) No longer can it be said that a dark band on light ground is discerned as the intensity within the geometrical image if the band has virtually the same mean value as the remainder of the field. Figure 2.8 (c) shows that only the phase breaks of the object, e.g. the edges of band $A$, are perceivable.

Let us take as example an epithelial cell of the tongue: in a powerful objective this is a rather large object the diffracted light of which does not extend much beyond the phase-plate. The imaged cell shows but little contrast with the remainder of the field. Only the edges and inner details of the cell are visible. The edges are seen as discontinuities denoted by $m$ and $m^{\prime}$ in Fig. 2.8(c). All inner details display images similar to those in Fig. 2.8(a). These resulis are shown diagrammatically in Fig. 2.9. Figure 2.9(a) shows a cross-section of the 
object which is an outline of index and thickness changes in the cell. Figure 2.9(b) shows the structure of the phase-contrasted image. The whole cell evinces but slight contrast in relation to the remainder of the field, the discontinuities originated by the cell edges appear in $m$ and $m^{\prime}$. The imaged inner details conform to their structure.

Making a phase-plate opaque brings about a central dark ground. Theoretically, contrast is maximal since the dephasing details are

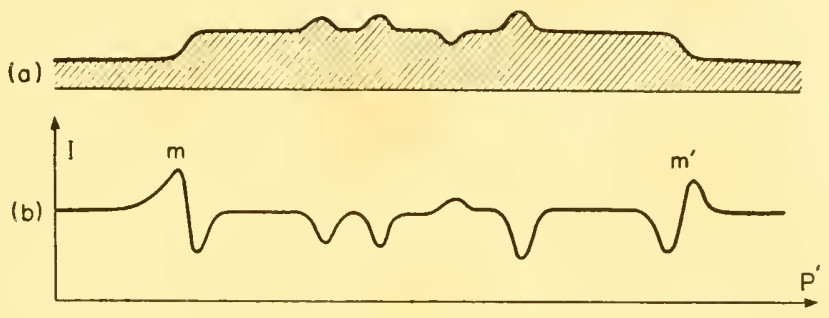

FIG. 2.9. Image structure of an extended object.

brightly outlined against a dark ground. However, this makes image interpretation more tricky. It should be borne in mind, that, in phasecontrast microscopy, intensities are proportional to the object-originated phase (equations 2.4 and 2.5) whereas those on dark ground are proportional to the squared phase. If the object dephases but slightly, viz. if $q$ is small, its square is still smaller. Therefore a dark ground requires intense sources that emphasize the minutest surface defects such as dust and so forth. Under such conditions the details displacing the phase inconsiderably are always clouded by the diffracted light, originated by improperly focused defects.

\section{PERCEPTION LIMIT AND RESOLVING POWER. EFFECTS OF STRAY LIGHT}

Let us consider an object not discernible in an ordinary microscope because it is transparent and not owing to its minute size. Therefore it exhibits no contrast with the remainder of the field. When observed in a phase-contrast microscope with an absorbing phase-plate, the imaged contrast is educed from expression 2.9. This expression implies that the microscope is perfectly devoid of stray light and the values so derived are therefore critical.

Let us consider a small transparent disk, giving rise to phase difference $\varphi$, and the diameter of which is, e.g. 4 to 5 times that of the diffraction disk. The image obtained in phase-contrast microscopy 
is given by expression 2.9. It can be considered that limit of perception is attained when its contrast attains 0.04. Then equation 2.9 gives

$$
\gamma=2 \gamma, N=0.04 \text {. }
$$

As the phase-plates generally used do not exceed $N=100$, then:

$$
\left(n-n^{\prime}\right) e=0.0002 \text {. }
$$

An object of thickness $e=1 \mu$ could only be seen, for instance, provided that the index differences between it and the medium surrounding it exceeds $2 \times 10^{-4}$. Stray light impairs sensitivity and its effects are the greater as phase-plate absorption increases. If intensity of the stray light is a fraction $K$ of the incident intensity, the image phase-contrast is divided by $1+N K$. When $N=100$ and $K=0.05$ the image contrast is divided by 6 . While the phase-contrast method does enable one to perceive details otherwise remaining invisible under normal conditions, its very principle is not conducive to improved resolving power. General diffraction laws, i.e. the structure of the diffraction pattern displayed by the objective, determine the resolving power of a microscope. The presence of the phase-plate alters but slightly the diffraction pattern. The phase-plate occludes a small portion of the central aperture of the microscope objective, thus slightly altering the diffraction pattern: the central disk is narrowed, thereby increasing the light in the diffraction rings surrounding it. The result is that the resolving power in the case of high-contrast imaged objects is slightly improved. Conversely, low-contrast imaged objects afford less improved resolution. A phase-contrast microscope provides approximately the same resolving power as an ordinary microscope. When observing a transparent object immersed in a medium the index of which is very similar to it, the details are not perceivable in an ordinary microscope. But, once the phase-contrast microscope has converted the phase changes into intensity variations, two discrete particles are no farther apart when resolving power is reached than with an ordinary microscope when observing a non-transparent object, featuring light-intensity variations.

\section{PHASE-CONTRAST MICROSCOPES IN TRANSNITTED I.IGHT}

Referring to Fig. 2.1, all the illuminating direct light passes through the aperture $S_{1}$ of the diaphragm $D_{1}$, located in the focal plane of condenser $C$. After passing through the condenser $C$ and the objec- 
tive $O_{1}, S_{1}$ is imaged at $S_{1}^{\prime}$ in the objective focal plane $F$. The direct light passes through the image $S_{1}^{\prime}$ of $S_{1}$. To dephase by $\pi / 2$ the direct vibrations in relation to the diffracted ones, all the light must penetrate in the phase-plate at $S_{1}^{\prime}$. The phase-plate, therefore, must overspread the imaged $S_{1}$ of the diaphragm $D_{1}$. If the phase-plate is ring-shaped, the diaphragm $D_{1}$ aperture should be a ring which, when imaged at $S_{1}^{\prime}$ does not overlap the phase-plate. When the latter is shaped as a small rectangular band, the aperture $S_{1}$ of the diaphragm $D_{1}$ should be a slit which, when imaged at $S_{1}^{\prime}$, is overspread by the phaseplate. When a linear-phase plate is used, objects shaped lengthwise, extending at right angles to the phase-plate, are not perceived. Such a strip affords a preferred direction of vision: merely rotating the object shows up details that would remain invisible in the initial position. Regardless of the phase-plate shape, it follows that segragation of the diffracted and direct light is unsatisfactory if the phaseplate is too wide. In Fig. 2.4, the beam of diffracted light overspreads the objective and lies in the $M M^{\prime}$ area in the focal plane $F$. The phase-plate area should be small in relation to that of the $M M^{\prime}$ diameter.

Figure 2.10 shows diagrammatically a phase-contrast microscope with a ring-shaped phase-plate. Diaphragm $D_{1}$, set in the focal plane

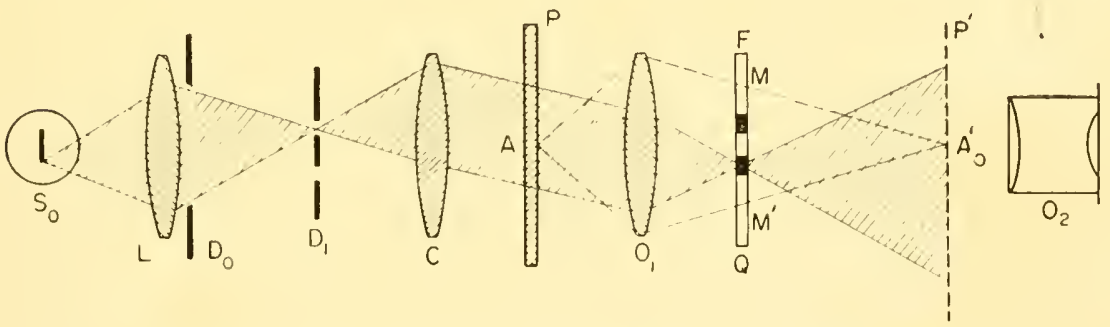

Fig. 2.10. Phase-contrast microscope with annular phase plate.

of the condenser $C$ is provided with an annular aperture. The imaged filament of the lamp $S_{0}$, adequately enlarged as to overspread the whole ring sections, is cast on the diaphragm ring. The annular phaseplate $Q$, located in the focal plane of objective $O_{1}$ overspreads the imaged ring $D_{1}$. The shaded area in Fig. 2.10 does not show the whole direct beam of light but only a source-generated beam of light. The beams originated from other points of the lamp $S_{0}$ filament follow similar paths but at different slopes. 
Fixed-characteristics phase-plate microscopes

In Zernike's original microscope the phase-plate was of collodion, immersed in Canada balsam and cemented between the two components of one of the objective doublets (Fig. 2.11). Let $n_{1}$ be the collodionplate index and $n_{2}$ that of the Canada balsam (dark area in Fig. 2.11),

(2) (1)

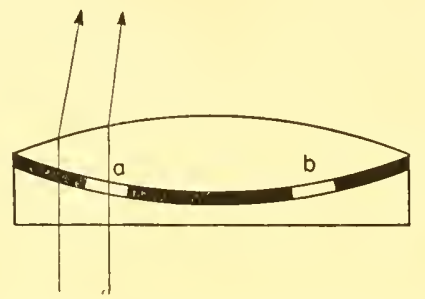

FIG. 2.11. Annular phase plate between two lenses of a microscope objeclive.

the thicknesses of the collodion plate and of the balsam are the same and equate $e$. To show the quarter-wave lag taking place between the ray 1 traversing the phase-plate and the ray 2 , passing next to it in the balsam, all that is needed is that the difference between indices $n_{1}$ and $n_{2}$ be given by the expression:

$$
\left(n_{1}-n_{2}\right) e=\lambda / 4 \text {. }
$$

In modern objectives, phase-plates are generally obtained by evaporation of thin layers in vacuo. One or several of such layers, such as $a$, are deposited on the surface of the objective-lens and evaporated

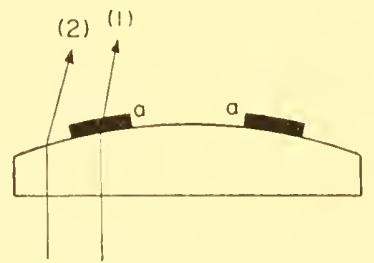

FIG. 2.12. Phase plate deposited on the surface of a lens by evaporation in racuo.

in vacuo (Fig. 2.12). Proper adjustment of the layer thickness achieves either a lag or lead equal to $\lambda / 4$ between the traversing ray 1 and the ray 2 passing next to it. Such chemicals as magnesium or cryolite are used in making transparent phase-plates and produce the required phase-displacement of $\pi / 2$. Absorption is achieved by evaporating 
above the previous layer a very thin metal layer that absorbs the light virtually without affecting phase displacement. Evaporation of the thin layers constituting the annular phase-plate is carried out on one of the lenses closest to the objective's focal plane. Figure 2.13 shows

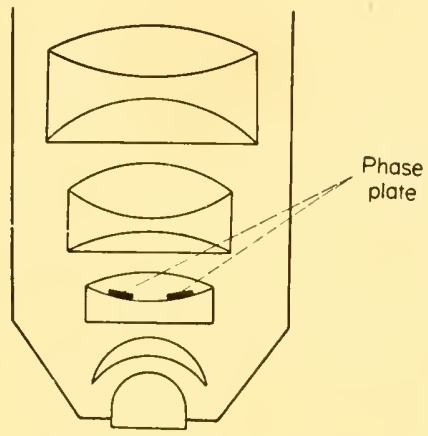

FIG. 2.13. Phase contrast immersion objective.

an immersion objective in which the phase-plate was evaporated on one of the components of a doublet prior to cementing. The objective's focal plane lies in this area.

In absorbent phase-plates made by means of thin metal layers, a not inconsiderable portion of the direct light is reflected by the phase-plate and becomes stray light. In Reichert's "anoptral" device,

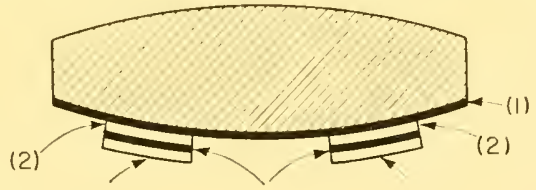

(4)

(3)

(4)

FiG. 2.14. Reichert's anoptral device: (1) and (4) no reflecting thin film; (2) dielectric thin film; (3) absorbing metallic thin film.

there is no stray light by reflection and the phase-plate may exhibit substantial absorption (Fig. 2.14). The "anoptral" phase-plate is featured by Number $N=15$. This phase-plate divides the incident intensity of the direct light by 15 and brings about a $\pi / 2$ phase difference. Adjustable annular-diaphragm microscopes

The characteristics of the foregoing phase-plates are permanent. If two objectives of identical geometrical characteristics are used seriatim, the annular diaphragm remains the same in both cases provided 
that the phase plate remains geometrically identical, too. Were such not the case, e.g. the objective's focal changed, the diaphragm ring must also be changed. This is why, in many microscopes, the condenser carries, close to its focal plane, a revolving sub-stage comprising rings of various sizes. A specific ring applies to every type of objective. Leitz has perfected a phase-contrast microscope in which the annular diaphragm of the condenser is substituted for a special condenser, devised by Heine, whereby a mere adjustment makes it suitable for any objective. Heine's condenser (denoted by $M$ in Fig. 2.15) is sub-

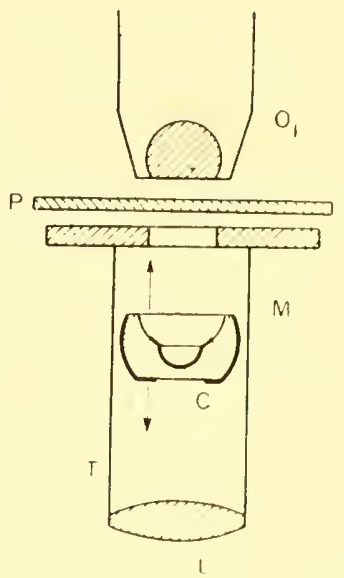

FIG. 2.15. Heine's condenser (Leily).

stituted for the standard one and set under the specimen $P$. It consits of the fixed lens $L$ and the optical system $C$, sliding within the drawtube $T$. The paths of the light-rays from the optical system $C$ are shown in Figs. 2.16(a) and 2.16(b). The light-rays from the lens $L$ (not shown in Fig. 2.16) are first reflected by the plane portion $b b$ and, next, by the annular mirror $d d$ and emerge at ee (Fig. 2.16(a)). The system $C$, consisting of a glass member, is shown by the dark area in Fig. 2.16. Except in the central area $x$ and the annular area ec, the faces of the system $C$ are made reflective by metallization. The annular area replaces the usual condenser diaphragm. The microscope's objective consists of the lenses $l_{1}, l_{2}, l_{3}$ and the phase-plate $Q$. In the position shown in Fig. 2.16(a), the system $C$ is rather far from the specimen, e.g. about 20 millimetres. This is why the lenses $I_{1}$ and $l_{2}$, the focal of which are short, can image the ring ee on the phaseplate $Q$ within the objective. 
Now, let us bring the system $C$ closer to the specimen (Fig. 2.16(b)). Owing to the distance from $C$ to $L$, the incident beam is reflected on to the convex mirror at $a^{\prime} a^{\prime}$ in an area more removed from the axis. Instead of travelling back to $b b$, the light is then reflected direct by

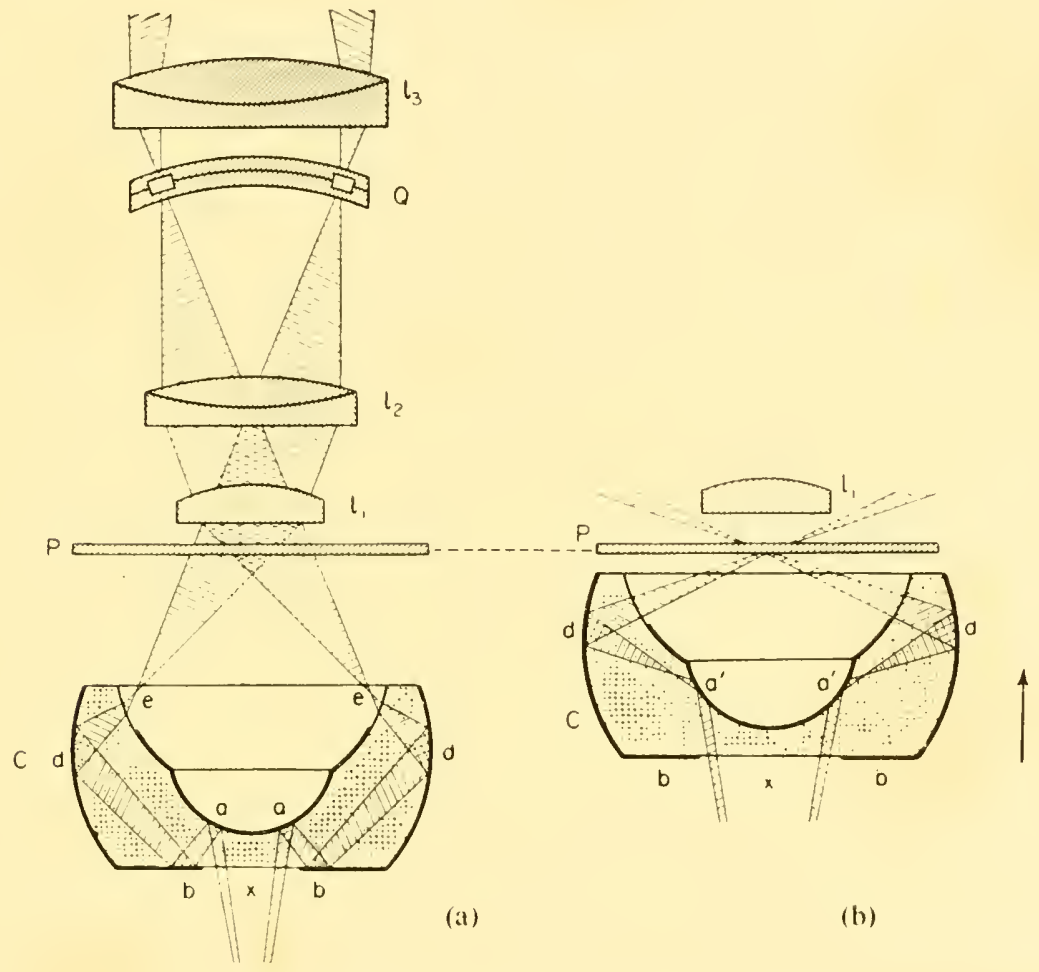

FIG. 2.16. Heine phase-contrast microscope (Leitz).

the annular ring $d d$. The emerging light-beams traverse the specimen at very wide angles and do not penetrate into the objective. The lightbeams diffracted by the specimen details are the only ones to be collected by the microscope's objective and a standard dark ground is thus obtained. In this way, shifting the system $C$ brings about a continuous change-over from the various following positions: light ground, phase contrast, oblique-illumination, light ground, dark ground. In Messrs. Officine Galileo's phase-contrast microscope, a pancratic system forms an image of variable dimensions from a fixed annular diaphragm. In this way the imaged ring dimensions are adjustable and made to match the phase-plates of the objectives involved. 
Microscopes with variable phase-plates

In Osterberg's polanret microscope, the phase-plate proper, denoted by $Q$, consists of two polaroid elements the axes of which cross each other (Fig. 2.17). The ring, shown in thick lines in Fig. 2.17, is cut

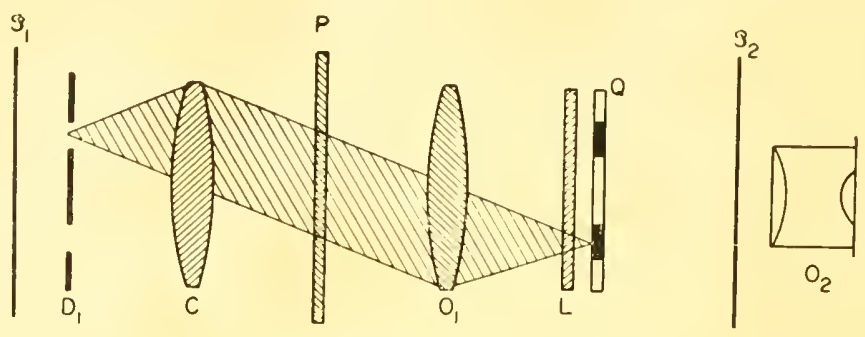

FIG. 2.17. Polanret microscope (after Osterberg).

out from a polaroid sheet. Two other polaroid elements the axes of which are paralleled and at right angles to the axis of the first polaroid element of the ring are on each side of the ring. These various elements form the phase-plate $Q$ just ahead of which is the quarter-wave plate the axes of which are $45^{\circ}$ from the axes of the polaroid elements of the plate $Q$. The polarizer $P_{1}$ and the analyser $P_{2}$ complement the device. Rotating the polarizer $P_{1}$ varies the phase difference between the direct and the diffracted vibrations. Rotating the analyser $P_{2}$ alters the amplitude's ratio of such vibrations while their phase differ-

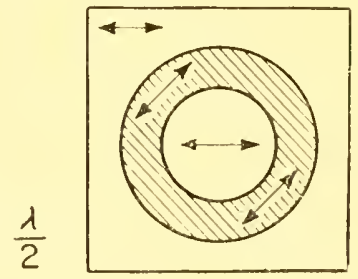

FIG. 2.18. The Kastler and Montarnal phase-plate.

ence remains constant. The result is an absorbing phase-plate adjustable ad libitum. The Kastler and Montarnal phase-plate consists of two half-wave plates the axes of which are $45^{\circ}$ from each other (Fig. 2.18), to which is added a quarter-wave plate one preferred direction of which is parallel to the direct vibration. Both plates are inserted between a polarizer and an analyser as shown in Fig. 2.17. Assuming that the polarizer generates a vibration directed along the 
axis of the half-wave plate forming the ring shown in Fig. 2.8, the direction of the vibration passing through this plate remains unaltered but the diffracted vibration traversing the other half-wave plate rotates through an angle of $90^{\circ}$. In the latter plate the direct vibration is $45^{\circ}$ from the half-wave plate it traverses. It is well known that a half-wave plate against which a recilinear vibration impinges supplies a vibration which emerges as a rectilinear and symmetrical in relation to the preferred directions. After passing through the half-wave plate, the direct and the diffracted vibrations are converted in two perpendicular vibrations. The $\pi / 2$ phase difference, required between the direct and the diffracted wave, is provided by the quarter-wave plate one of the preferred directions of which is parallel to the direct vibration. Merely rotating the analyser alters at will the ratio of the direct and the diffracted amplitudes. The outcome is an absorption-adjustable phase-plate.

Hartley devised an arrangement comprising two quarier-wave plates $45^{\circ}$ from each other (Fig. 2.19). Now, if the incident vibration is parallel to the axis of the quarter-wave plate forming the ring, the direct light is linearly polarized and the diffracted light circularly.

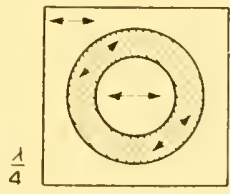

FIG. 2.19. The Hartley phase-plate.

Rotating the analyser varies the direct light-intensity without affecting that of the diffracted light. Phase difference is obtained by some means or other, e.g. deposits of thin layers on the ring.

The rotatory power of quartz has been used by Taylor to vary phase-plate absorption. As in the foregoing methods, the incident light is linearly polarized; the direct light passes through the dextrorotatory quartz plate $Q_{1}$ and the diffracted light through the two levorotatory quartz plates $Q_{2}$ (Fig. 2.20). These quartz-crystals make up a parallel-sided plate placed, as usual, in the imaged plane of the radiant. The direct and the diffracted vibrations rotate $45^{\circ}$ in a different direction and meet $90^{\circ}$ from each other at the plate outlet. As in the foregoing methods, rotating the analyser varies the direct and the diffracted amplitudes. Next, the phase difference is obtained 
by some means or other, e.g. evaporation of thin layers on the ring $Q_{1}$.

B. O. Payne reverted to the principle of half-wave plates in order to sunder the direct and the diffracted light in two perpendicular components. Behind the plate is a Bravais compensator whereby the phase difference can be varied continuously. Absorption is varied by rotating the analyser.

Locquin's phase-plate consists of the half-wave Cellophane ring $a b$, cemented to Canada balsam between the two elements of the objective's doublet (Fig. 2.11). The incident light is polarized $45^{\circ}$ from

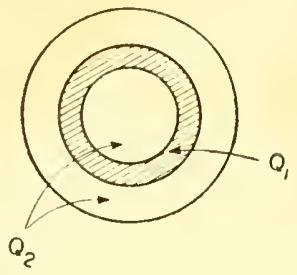

FIG. 2.20. The Taylor phase-plate.

the Cellophane-ring axis. The direct light (1) after passing through the ring vibrates $90^{\circ}$ from the diffracted light (2) the direction of which remains unaltered. Intensity of the direct and the diffracted light is varied by rotating the analyser. The phase-difference is obtained by adjusting the index differences of the Cellophane and the Canada balsam.

In another layout, Locquin suggested making use of interference filters (Varicolor-Wild). The phase-ring is made up of stacked, evaporated-in-vacuo thin layers. Such an arrangement is an interference filter letting through but a narrow spectrum band $(60 \AA)$. The layers are so arranged that a ray passing through the ring is phase-displaced by $\pi / 2$ in relation to a ray passing next to it. The interference filter acts concurrently as a phase-plate. When such a plate is illuminated with the radiation for which the interference filter is designed, the outcome is a low-absorption phase-plate. A slight wave-length change in the incident light increases filter absorption. The outcome is a variable-absorption phase-plate with monochromatic illumination the wave-length of which can be varied.

\section{Transferred-image phase-contrast microscopes}

Figure 2.2I shows the diagram of the Françon-Nomarski device. The image $S_{1}^{\prime}$ of the diaphragm $S_{1}$, after traversing the condenser $C$ 
and the objective $O_{1}$, is collected by the projection-eyepiece $L$ that images it at $S_{1}^{\prime \prime}$, on the phase-plate $Q$. The image $P^{\prime}$, of the specimen $P$, is collected by $L$ which forms an image $P^{\prime \prime}$, after being refiected on $Q$. This is the image perceived in the eyepiece $O_{2}$. The phase-plate $Q$

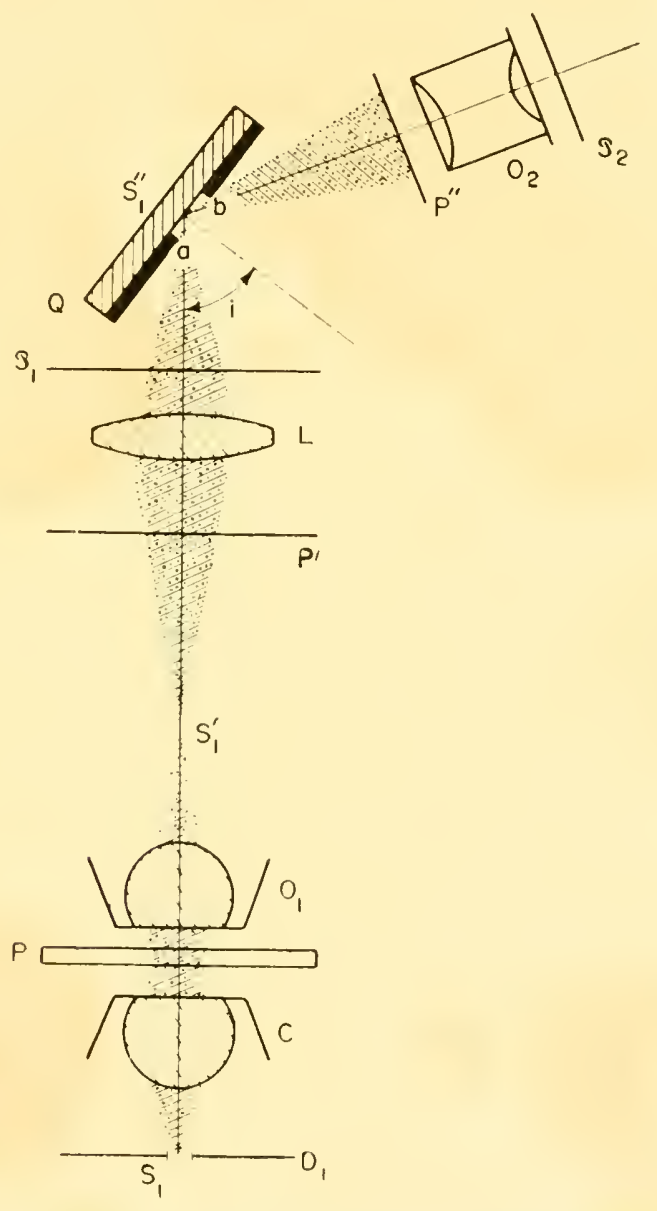

FIG. 2.21. The Françon-Nomarski device (Nachet).

consists of a glass plate whose face against which the light impinges is flat, well polished and coated with a metal layer evaporated in vacuo. Provision is made to remove metal in slit-shaped area of width $a b$. In Fig. 2.21 this slit lies lengthwise at right angles to the plane of the figure. The diaphragm $S_{1}$ is a slit, too, its image $S_{1}^{\prime \prime}$ being overspread by $a b$. Thus the direct light is reflected on to the glass at $a b$ while 
the diffracted light is almost wholly reffected by the metal layer. Suitable determination of the metal-layer thickness and taking the incidence $i$ in consideration, the $\pi / 2$ phase difference is obtained. Owing to the reflection on the glass at $a b$, the direct light is polarized: if $i=57^{\circ}$ (Brewster's incidence), polarization is complete. The diffracted vibrations reflected on the metal are virtually unpolarized. Then a polarizer is set at $P_{1}$ ahead of the phase-plate $Q$ and an analyser $P_{2}$ next after. Both direct and diffracted vibrations are polarized identically and may interfere. $P_{1}$ is set so that the incident vibration is $45^{\circ}$ from the phase-plate plane of incidence. Rotating the analyser $P_{2}$ brings about a variable-absorption phase-plate with either positive or negative phase-contrast.

In B. O. Payne's device (Fig. 2.22) the prism $R$, next after the objective $O_{1}$, reflects the light on to the concave spherical mirror $P^{\prime}$

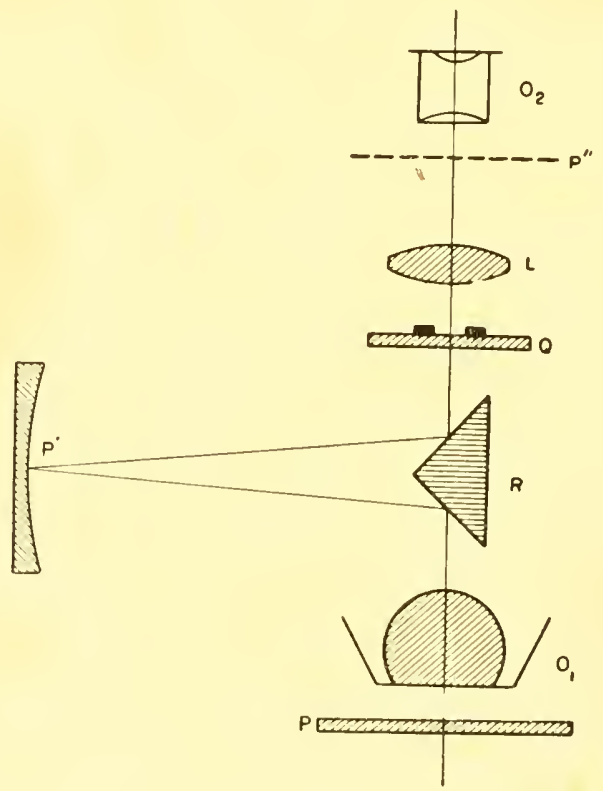

FIG. 2.22. B. O. Payne's device (Cooke, Troughton and Simms).

The radius of curvature of the latter is such that it images the focal plane of the objective $O_{1}$ at $Q$, following reflection on $R$. The phaseplate is at $Q$ and the specimen imaged by the objective is close to the mirror $P^{\prime}$. The optical system $L$ gives at $P^{\prime \prime}$ a new image, perceived by the eyepiece $O_{2}$. Such devices are particularly convenient 
when using variable-characteristics phase-plates. In powerful objectives, reaching the focal plane, lying in the objective itself, is hardly feasible. Designing a phase-plate, varied in the objective, is no easy matter whereas the converse is true if the focal plane is imaged outside the objective in an accessible area. Zernike was the first to use this image transfer in the first phase-contrast microscope he designed.

Instead of placing the phase-plate next after the objective, it occurred that it might be placed ahead of it. In Françon's half-sphere, the specimen is at $P$ (Fig. 2.23) and the detail $A$ close to the centre

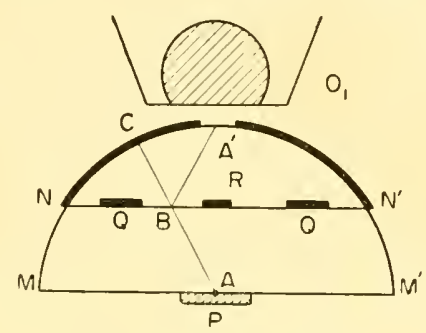

FIG. 2.23. Françon's half-sphere.

of the glass half-sphere $M N A^{\prime} N^{\prime} M^{\prime}$ which is a metallized reflecting layer at $N A^{\prime} N^{\prime}$. A small quantity of metal was removed at $A^{\prime}$. The half-sphere was split in two at $N N^{\prime}$. On the upper-half surface $N N^{\prime}$ a thin semi-reflecting layer was deposited. The annular phase-plate is at $Q$ and a small opaque screen at $R$. The half-spheres were then cemented.

The condenser (not shown in Fig. 2.23) has, in its focal plane, an annular diaphragm on which the source is imaged as usual. After passing through the condenser, the specimen being reflected on the spherical mirror $N A^{\prime} N^{\prime}$, the annular diaphragm is imaged on the phase-ring $Q$. A ray of light, diffracted by $A$, travels along the path $A B C B A^{\prime}$ and the specimen is imaged at $A^{\prime}$. This image is of the same size as the specimen's and stigmatic because $A$ lies in the centre of the sphere $N A^{\prime} N^{\prime}$. After travelling along an identical path, the direct light passing through the phase-plate $Q$ ends at $A^{\prime}$. The image $A^{\prime}$ is therefore a phase-contrast image, observable in an ordinary microscope. The object of the opaque screen $R$ is to stop the rays of light from entering the objective after straying from the path shown. Provided the device be soundly designed, highly absorbing phase-plates may be used as the direct light is stopped before penetrating the objective and it does not originate any stray light. 


\section{Colour phase-contrast microscopes}

F. Zernike, who invented phase-contrast microscopy, was also the first to investigate colour phase-contrast potentialities. Making use of the dispersive properties of thick phase-plates is the basis of the method. It was thoroughly studied by C. P. Saylor, A. T. Brice and F. Zernike. They use a phase-ring set on one of the objective lenses. The space not taken up by the ring is coated with a thin layer of another substance. These two substances have the same index in the spectrum centre $(0.555 \mu)$ but different dispersion. In the violet, the phase-ring refraction index is lower than the adjacent layers and higher in the red. Therefore the positive or negative phase-contrast is the same for the selfsame object according as to whether the specimen is illuminated by either of the two spectral areas spaced apart by a $0.555 \mu$ wave-length. Let us consider, for example, an object the refraction index of which exceeds that of the ambient medium: it will be brighter or darker than the remainder of the field in red and bluc light respectively. In white light the object is yellow-orange, edged with bluish purplc.

Barer has shown a type of colour phase-contrast based on the use of dichroic filters.

Locquin's Varicolor yields a coloured phase-contrast. All that is required is to illuminate the specimen with two suitably combined colours to obtain a positive phase-contrast image of one of the colours and a negative phase-contrast image of the other.

In Françon's colour phase-contrast, a quartz plate cut $90^{\circ}$ from the axis (not shown in Fig. 2.21) is inserted between the polarizer $P_{1}$ and the phase-plate $Q$. The quartz plate is $1.87 \mathrm{~mm}$ thick and causes a $45^{\circ}$ rotation of the yellow. If the polarizer is $45^{\circ}$ from the incidence plane, the yellow is overshadowed through glass reflection (Brewster's law) whereas such is not the case in the metal-reflected diffracted light. Various combinations are feasible by altering the condenser orientation or the quartz thickness.

Grigg devised a colour phase-contrast microscope featuring an objective fitted with an ordinary phase-plate: he substituted the condenser diaphragm for two suitably coloured filters. The device is an extension of Rheinberg's conventional system to phase-contrast. Barham suggested using two phase rings illuminated by two radiations. The observed image is the outcome of superimposing two differentlycoloured phase-contrasted images. 
Phase-contrast microscopes in ultra-violet and infra-red radiations

Discounting construction problems, the phase-contrast method is applicable to the ultra-violet and infra-red radiations just as it is to the visual range. Yet it should be noted that transparent objects in the visual range may become absorbent in the ultra-violet and

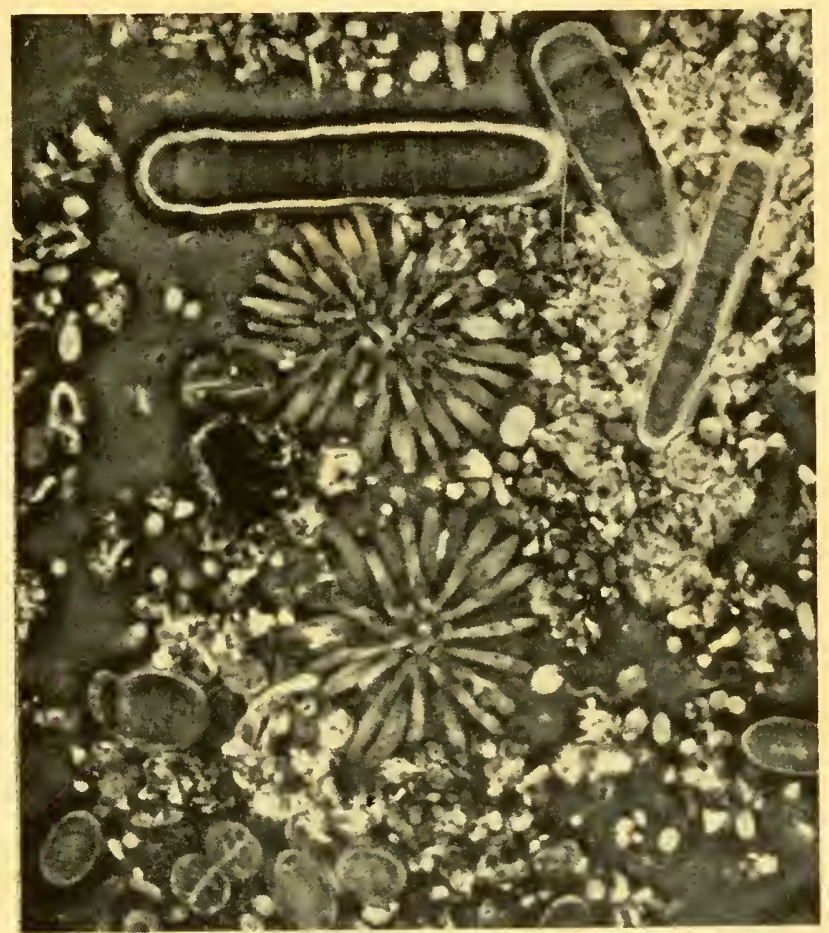

FIG. 2.24. Selection of organism including the rosette Oscillospira, Guilliernondii, sarcina, Quin's organism and selenomas. Ultra-violet negative phase-contrast. J. Smiles and M. J. Dobson (courtesy of Cooke, Troughton and Simms).

infra-red radiations. This applies to many biological specimens: in ultra-violet light some parts of living cells are absorbent and, when phase-contrast imaged, are more tricky to interpret. The imaged contrast is the outcome of incident radiations absorbed by the substance and a phase-contrast effect. These two effects are not necessarily additive: let us consider a slightly-contrasted non-dephasing detail in ultra-violet radiation. Applying phase contrast converts low-contrast, non-dephasing objects into transparent ones. 
The works published related to phase-contrast in ultra-violet and infra-red radiation are few: Bennett, Woernley and Kavanagh, in the USA, Taylor in England, have described and used phase-contrast microscopes in ultra-violet radiation. Barer has constructed an infrared device comprising an electron image converter. Figure 2.24 shows a photograph made with the ultra-violet phase-contrast microscope designed by Cooke, Troughton and Simms.

\section{PHASE-CONTRAST APPLICATIONS}

Phase-contrast applications in the various branches of Science and Industry are manifold. Only a few such applications are briefly outlines herein.

In cytology, phase-contrast microscopy permits observation of cells, tissue fragments and histological-preparation sections. Cell nuclei and protoplasmic structures are positively identified. Ludin showed that phase-contrast microscopes show chiefly the cell nucleus and membrane whereas staining methods evidence the chromatin structure.

Extensive phase-contrast research has been made in connection with the cytoplasmic structure, mitochondria, fibroplast spindles, the Golgi apparatus, living cells and so forth.

Albertini made extensive research with phase-contrast microscopes to investigate and diagnose tumours.

In haematology, extended observation of blood and medulla cells was made by means of phase-contrast microscopy.

It should be noted that Zernike's method applies equally well to animal and plant cytology. Research pertaining to the cells of algac and metaphysical plants has been carried out, too.

Phase-contrast microscopy has also provided valuable results in the study of parasitic worms (egg growth), in diagnosing dermatomycosis in mycology and observation in vivo of bacteria.

Particularly useful applications of phase-contrast microscopy are those in the fields of chemistry, crystallography and mineralogy. Colourless crystals having an index different from that of the medium encompassing them are typical objects for phase-contrast microscope study. For example, the phase-contrast definition of paraffin microcrystals in alcohol excels that of polarized light. The latter process only shows the crystal edges whereas in phase-contrast work they appear as thin strips evincing hexagonal symmetry (Bennett). Phasecontrast microscopy is particularly suitable for some crystal-growth 


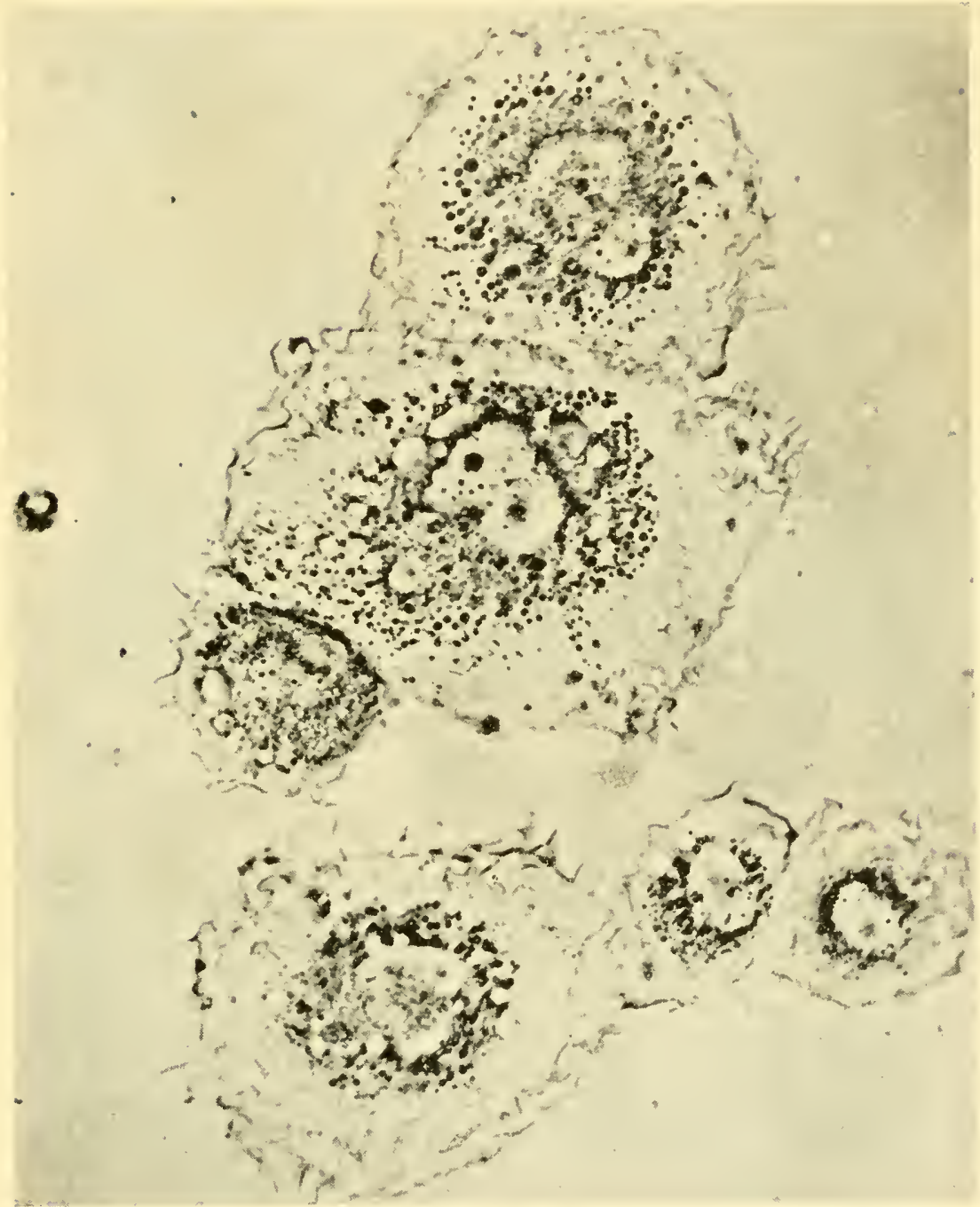

FIG. 2.25. Rat Carcinoma - Bright contrast-medium (courtesy of Dr. O. Gey, American Optical company).

problems as the sequence of phenomena is readily observable. The same applies to investigation of impurities in oil emulsions. Much information regarding the structure of textile fibres is also gained by means of this method which readily discloses minute inclusions, cracks and the like. Rayon fibres and their cross-sections are conveniently 


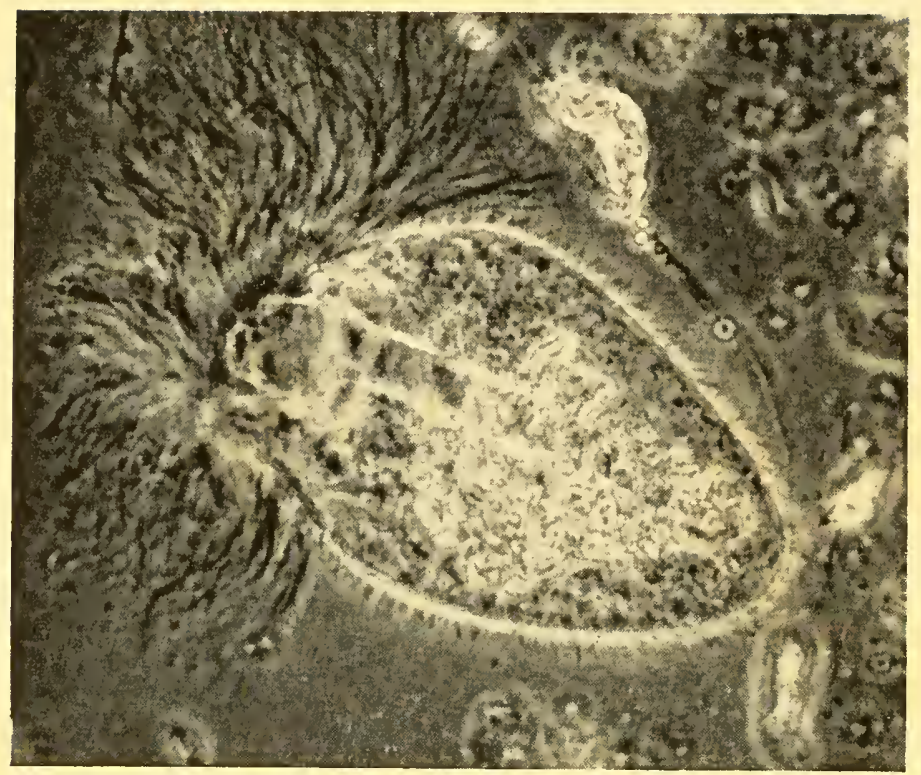

FIG. 2.26. Symbiote of a termite. Wild phase contrast microscope (courtesy of Wild).

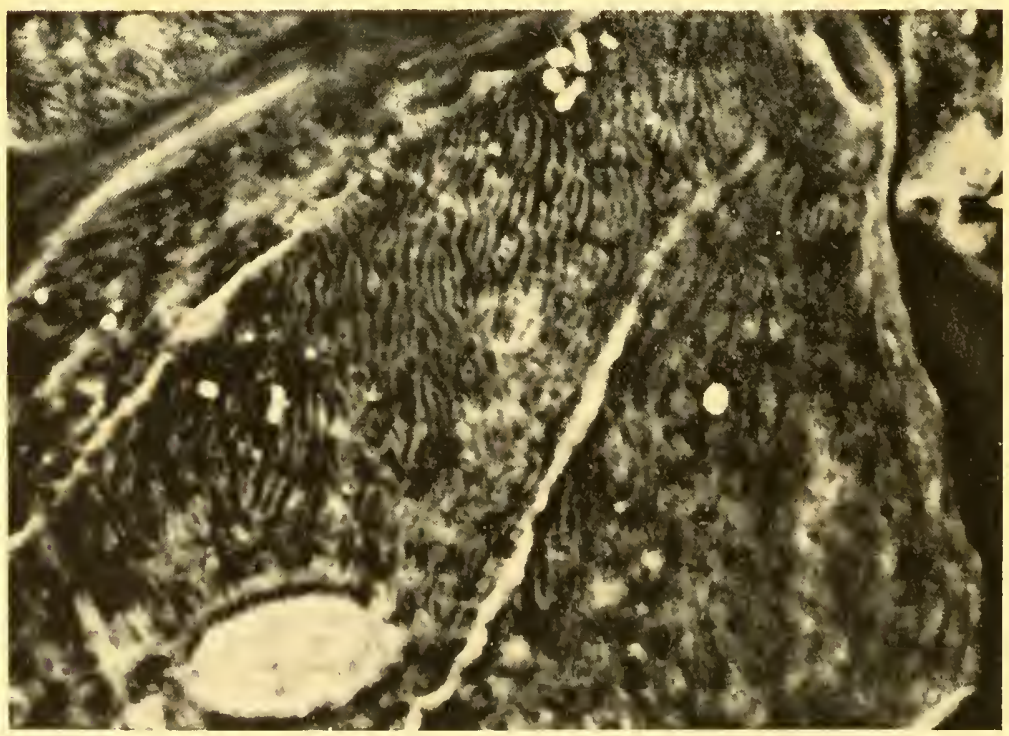

FIG. 2.27. Epithelial cell-Reichert's anoptral device. 
observed by dipping them in paraffin oil. Figures $2.25,2.26$ and 2.27 show several phase-contrast micrographic samples.

To sum up, while phase-contrast potentialities are considerable in many fields, it requires special regard if full sensitiveness of the process is to be taken advantage of. Specimens are to be as thin as possible: thicker specimens always yield unsatisfactory images which are difficult to construe. 


\section{Interference Microscopy in Transmitted Light}

\section{OBJECT AND FUNDAMENTALS OF INTERFERENCE MICROSCOPY}

THE object of interference microscopy is the same as that of phasecontrast microscopy: it consists in detecting transparent objects remaining unseen not owing to their minute size but to their lack of contrast in the remainder of the field. Phase-contrast microscopy provides outstanding sensitiveness when observing very minute path differences; the imaged objects conform accurately when the latter are narrow. This type of method is based on taking effect on the direct light without altering the light diffracted by the detail. This is not feasible unless the detail observed is small: otherwise, the light it diffracts is coalesced with the direct light to such an extent that segregating readily the two phenomena is not possible. Furthermore, the phase-plate occludes the objective to a greater or lesser degrec, this giving rise to a diffraction halo edging the images.

Interference-microscopy fundamentals are different. The phaseshifting object is placed in an interferometer and alters the optical path of the light-rays passing through it. As in any interference phenomenon, an optical-path change correlates a light intensity change rendering visible the transparent object. Owing to its very principle, interference microscopy does not discriminate between wide and narrow objects: phase-shifting objects are seen regardless of shape and dimensions.

However, these advantages are often offset by the instrument's complexity and the adjustment skill required.

\section{FUNDAMENTALS OF TWO-IVAVE INTERFERENCE MICROSCOPES}

Interference-microscopy fundamentals may be outlined as follows (Fig. 3.1). The condenser-originated light-ray $S M$ originates at $M$ the two rays $B$ and $A$. The branching taking place at $M$ is brought about 
by one of the interferometer's elements. The light-ray $M A N$ passes through the phase-shifting object $A$ while the ray $M B N$ passes next to it. The other interferometer element causes these two rays to join again at $N$ into a single ray which then passes into the microscope. If the object $A$ is not there, the different paths of the two light-rays $M A N$ and $M B N$, difference controlled by the interferometer, has a definite

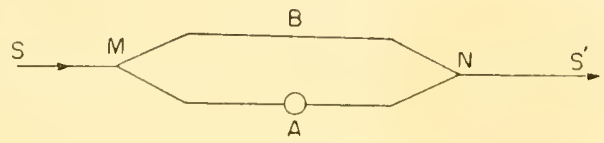

FIG. 3.1. Principle of an interferometer microscope.

value. Intensity of the $N S^{\prime}$ ray is determined by the interferences of the rays $M A N$ and $M B N$. Letting in the transparent object $A$ on the $M A N$ path, for example, alters the difference between the paths $M A N$ and $M B N$. The result is an alteration of the interference between these two rays giving rise to a change in intensity that brings out the object $A$. Referring to the sinusoidal aspect of the vibrations, as was done in the phase-contrast section, shows how this phenomenon occurs.

The sinusoid (1) in Fig. 3.2 shows the vibration along the path $M B N$ of Fig. 3.1. Let us assume that the sinusoid (2) shows the vi-

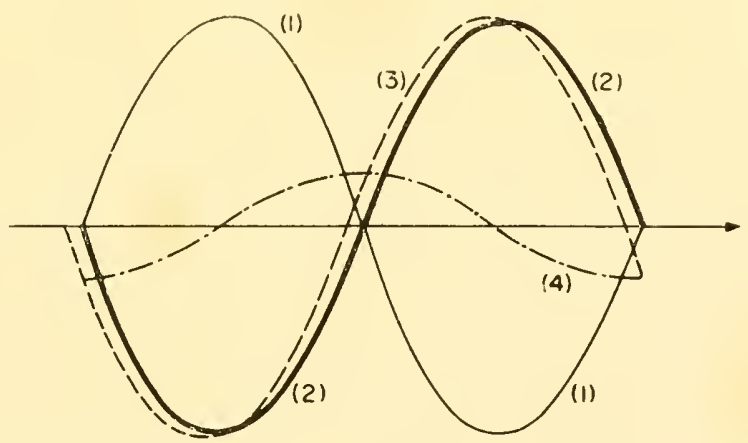

FIG. 3.2. Vibrations diagram in interference microscopy.

bration along the path $M A N$ when there is no object. The arrangement will be that shown in Fig. 3.2 provided the difference between the $M B N$ and $M A N$ paths equates an odd number of times $\lambda / 2$. As shown in Fig. 3.2, both vibrations ( 1 and 2 ) will then be in opposition. Suitable interferometer adjustment can provide such an arrangement. 
From $N$ onwards (Fig. 3.1) these two vibrations coalesce cancelling themselves out in the process since they are in opposition.

The field of view is completely dark.

If the object $A$ is there, the vibration (2) lags a little and becomes the sinusoid (3) in Fig. 3.2. In the phase-contrast section, it was mentioned that such a vibration could be considered as the sum of the two vibrations (2) and (4) which are shifted by one quarter wave. Now the vibration (2) cancels itself out with (1) and only the vibration (4) remains: the vibration diffracted by $A$. The object is bright against a dark ground. The interference method provides a dark ground without occluding the light-beams and, therefore, without a spurious diffraction fringe. Let us now consider a path difference between the two paths $M B N$ and $M A N$ (without $A$ ) that no longer equates an odd number of times $\lambda / 2$ (in opposition vibrations), although remaining close to such value.

The sinusoid (1) of Fig. 3.3 shows the vibration travelling along $B M N$ and the vibration (2) along the objectless path $M A N$. These two combined vibrations give rise to the low amplitude vibration (3).

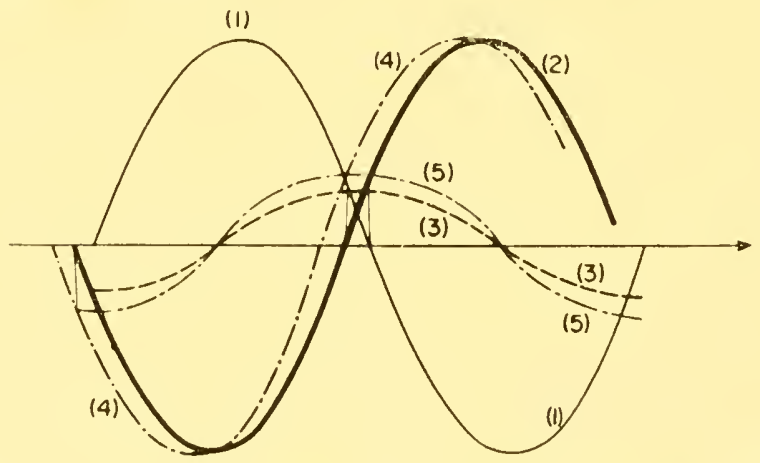

FIG. 3.3. Vibralions diagram in inlerference microscopy.

The amplitude of the latter is the luminous amplitude of the ficld next to the object. When the object is present, the vibrations (1) and (4), travelling along the paths $M B N$ and $M A N$, respectively, and passing through the object are to be compounded. In the same way, the vibration (4) is split in the two vibrations (2) and (5). Now, the vibration (2), combined with the vibration (1), generates the vibration (3). Therefore, the two vibrations travelling along the $M B N$ and $M A N$ paths (the object being present) amount to the two in-phase vibrations (3) and (5). 
To sum up, there are the following vibrations:

(a) The vibration (3), defining the luminous amplitude next to the object;

(b) The vibration (3) and (5) which, combined, define the luminous amplitude in the object.

The phenomenon is similar to that of phase-contrast. The amplitude in the imaged object equates the summed amplitudes of the vibrations (3) and (5). The amplitude of the vibration (3) is adjustable at will: if (2) exactly in opposition to (1) the ground is dark as (3) is zero, but the required amplitude of the vibration (3) can be obtained by altering slightly, by means of the interferometer, the phase difference between (2) and (1). Phenomena occur as if a phase-plate, adjustable for phase-displacement and absorbence, were used. Up to now, we did not give any clues as to interferometer design, i.c. how dissociation and recombination of the light-rays occur at $M$ and $N$ (Fig. 3.1).

There are many types of such instruments amongst which are those of Dyson, Krug and Lau (cf. Chapter IV), Philpott, Nomarski.

\section{DYSON'S INTERFERENCE MICROSCOPE}

Figure 3.4 is a diagram of Dyson's microscope. Let us consider the light-ray $S$ from the condenser (not shown in Fig. 3.4). This ray passes through the parallel-sided glass plate $L_{1}$ whose upper face is semi-metallized and on whose surface the ray branches off in two sections: one towards the incident ray, the other reflected at $M$ towards the instrument's lower part. The former ray passes first through the specimen $P$ at $A$ where the object lies and also through the second plate $L_{2}$ of same thickness as $L_{1}$, whose faces $x_{2} x_{2}^{\prime}$ and $x_{3} \cdot x_{3}^{\prime}$ are semi-metallized. It is reflected at $C$ on the upper face of $L_{2}$, then at $N$ on the lower face and along the path $N E F$. The latter ray reflected at $M$ towards the instrument's lower part is now reflected on to the lower part of $L_{1}$ at $B$. In this area, the plate $L_{1}$ is coated with a small, highly-reflective opaque-silvered disk. After $B$, the light-ray travels along $B N$, parallel to $M C$. The two rays $M A C N$ and $M B N$ rejoin at $N$ and merge with the $N E F$ ray. Figure 3.5 shows separately how the foregoing paths tally with the basic diagram (Fig. 3.1). It follows that the object is not illuminated solely by the light-ray just described but by an infinity of such rays, bunched cone-like from the condenser. Figure 3.4 shows the path of a ray symmetrical to the foregoing ray in relation to the system's axis. Since the specimen $P$ is between the 
plates $L_{1}$ and $L_{2}$, it could not be reached with a short-focus powerful objective. The light emerging from the plate $L_{2}$ is then taken over by a half-sphere. The $N E F$ ray proceeds in a glass of the same index

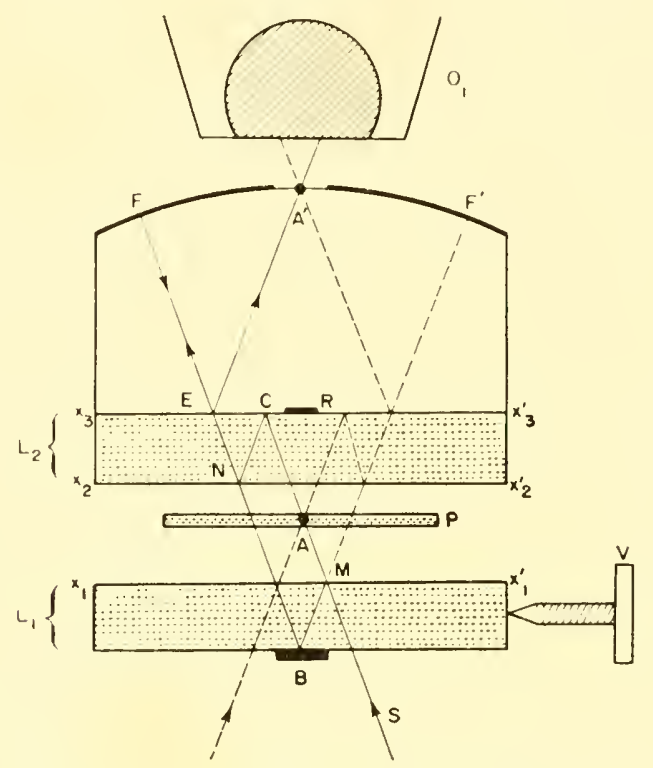

FIG. 3.4. Dyson interference microscope.

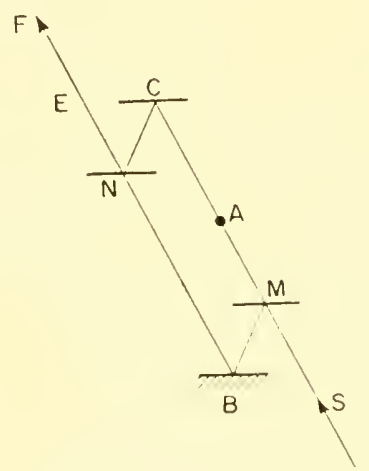

FIG. 3.5. Basic diagram of the ray paths in Dyson interference microscope.

as $L_{1}$ and $L_{2}$ which is bounded by the reflecting sphere $F A^{\prime} F^{\prime}$ (B centre). The $N E F$ ray is reflected at $F$ on to this concave spherical mirror and doubles back on itself to be reflected anew on to the semi-reflecting surface $x_{3} x_{3}^{\prime}$. It cnds ultimately at $A^{\prime}$ symmetrically with $B$ in relation 


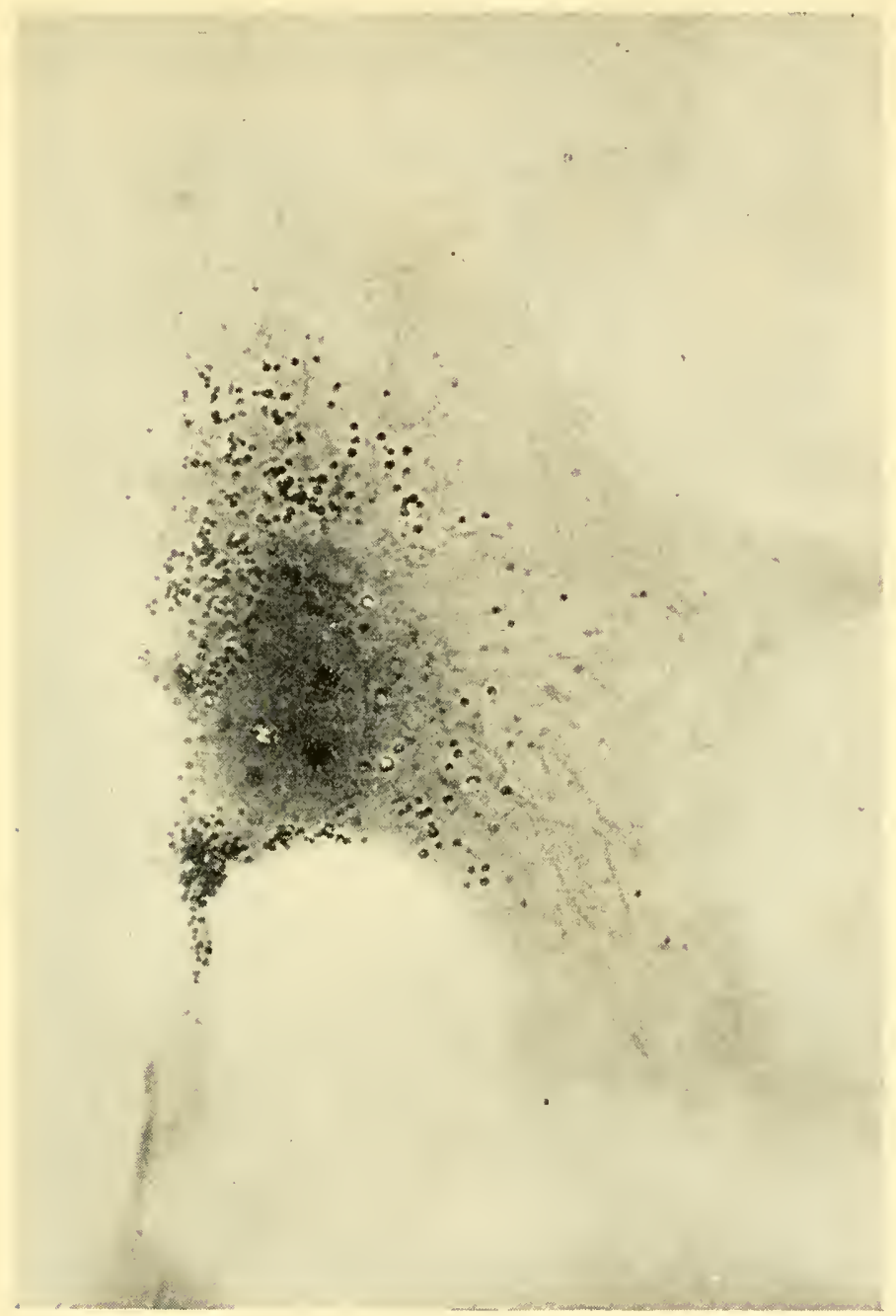

FIG. 3.6. Living fibroblast from mouse heart in tissue culture by bright-field interference contrast. $\times 1700$. Dr. H. G. Davies, King's College (courtesy of Cooke, Troughton and Simms).

to the surface $x_{3} x_{3}^{\prime}$. The light-ray cone converging in $A$ on to the object, converges anew in $A^{\prime}$ in an area where the reflecting metal layer was removed beforehand.

Therefore, $A$ is efficiently imaged at $A^{\prime}$ as phenomena occur as if $A$ were in the centre of the sphere $F A^{\prime} F^{\prime}$ and, of course, a sphere is stigmatic in its centre. 
The image $A^{\prime}$ (all the transparent details of which are perfectly visible owing to interference) is observed through the objective $O_{1}$ of an ordinary microscope.

If the instrument is constructed as described above, the path difference between the two rays $M A N$ and $M B N$ is zero provided the object is not present. Both rays are in phase and intensity at its peak. Let us bring in the specimen, i.e. the object: the path difference is no longer zero for the rays having passed through the object $A$, and the intensity in the image $A^{\prime}$ is lower than in the remainder of the field. The object is dark, to a greater or lesser degree, on a bright ground. In fact, both plates $L_{1}$ and $L_{2}$ are not strictly parallel-sided plates but very-small-angled prisms. If the slopes lie in the same direction, edges of the prisms being parallel, phenomena occur as in the foregoing. The field is uniform. The plate $L_{1}$ is shifted by means of the screw $V$ and the path $M A C N$ in relation to path $M B N$ is adjustable at will. If, through motion of $L_{1}$, the ray $M B N$ passes through the plate $L_{1}$ in an area where it is thicker than $L_{2}$, the path $M B N$ is longer than that of $M A C N$ and the path difference between the two is adjustable as mentioned above. Let us assume this path difference to equate an odd number of times $\lambda / 2$ : both rays $M B N$ and $M C N$ are in opposition. The field of view is dark except in the area where the object $A$ lies, as the rays having passed through the latter are no longer in opposition with rays such as $M B N$. The typical image exhibits a dark ground. Instead of adjusting the path difference so that the paths $M E N$ and $M C N$ (without object) are exactly in opposition, let us bring in between them a slight additional path. Phenomena occur as if it were a typical phase-contrast image. Actuation of the screw $V$ will vary contrast at will. If the sides of both plates $L_{1}$ and $L_{2}$ slope in opposite directions, the path-difference varies continuously from one end of the field to the other. Straight, parallel and equidistant fringes are developed. Actuation of the screw $V$ shifts all these fringes and the path is varied. It should be noted that, owing to the feasibility of adjusting path difference to very low values the instrument may be illuminated in white light.

When describing Fig. 3.4 no other reflections but those discussed above were taken into consideration. In the plate $L_{2}$, for instance, there are other reflections besides those at $C$ and $N$; many reflections take place but the relevant images are not in the same plane as image $A^{\prime}$ on which the microscope objective $O_{1}$ is focused. These images all have a dark centre caused by the diaphragm effect of the opaque 
reflecting screen $B$. The dark areas are amply adequate to cover the whole field of view so that these images do not bring about a loss in contrast of the image observed but only an inconsiderable loss of light.

In Dyson's interference microscope just described, dissociation and recombination of the beams takes place ahead of the microscope proper. The interferometer is not integral with the microscope. However, the microscope can be integral with the interferometer as in the case in Philpott's "spirocyclic" interference microscope or in Nomarski's anti-parallel-wave device.

\section{LEITZ'S INTERFERENCE MICROSCOPE}

Leitz's interference microscope is based on a similar system to the Mach-Zender interferometer (Fig. 3.7). The light from the source $S$ is split by the semi-reflecting plate in two beams (1) and (2). The former passes through $L_{1}$, is reflected on the mirror $M_{1}$, proceeds through the object $P$ and the objective $O_{1}$, is reflected on to the semireflecting plate $L_{2}$ and passes into the eyepiece $O_{2}$. The beam (2)

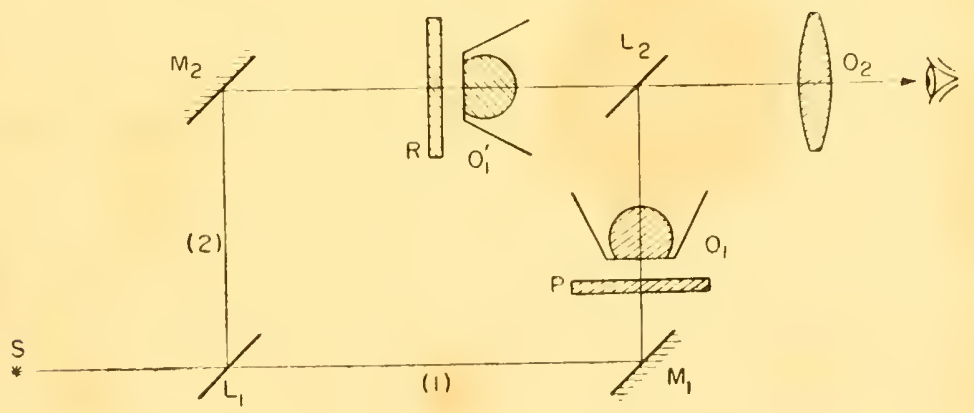

FIG. 3.7. Principle of the Mach-Zehnder interferometer applied to interference microscopy.

is reflected by the plate $L_{1}$, the mirror $M_{2}$, passes through an empty reference specimen, the objective $O_{1}^{\prime}$, the plate $L_{2}$ and penetrates into eyepiece $O_{2}$. The phase-shifting details of the specimen $P$ are observed by means of the interferences taking place between (1) and (2). If the phenomena are to be observed in white light both optical paths are to be identical. In particular the objectives $O_{1}$ and $O_{1}^{\prime}$ are to consist of identical members, glass thicknesses, indices and so forth. Figure 3.8 is a diagram of Leitz's interference microscope. The mirrors $M_{1}, M_{2}$ the plates $L_{1}, L_{2}$ are substituted for the combined prism $Q_{1}, Q_{1}^{\prime}, Q_{2}, Q_{2}^{\prime}$. 
The objectives $O_{1}$ and $O_{2}$ are corrected for infinity and the lens exhibits two superimposed images of $P$ and $R$ that are observed in the eyepiece $\mathrm{O}_{2}$. The condenser (not shown in Fig. 3.8) is at $C$. The path difference between the two paths (1) and (2) is varied by means of the

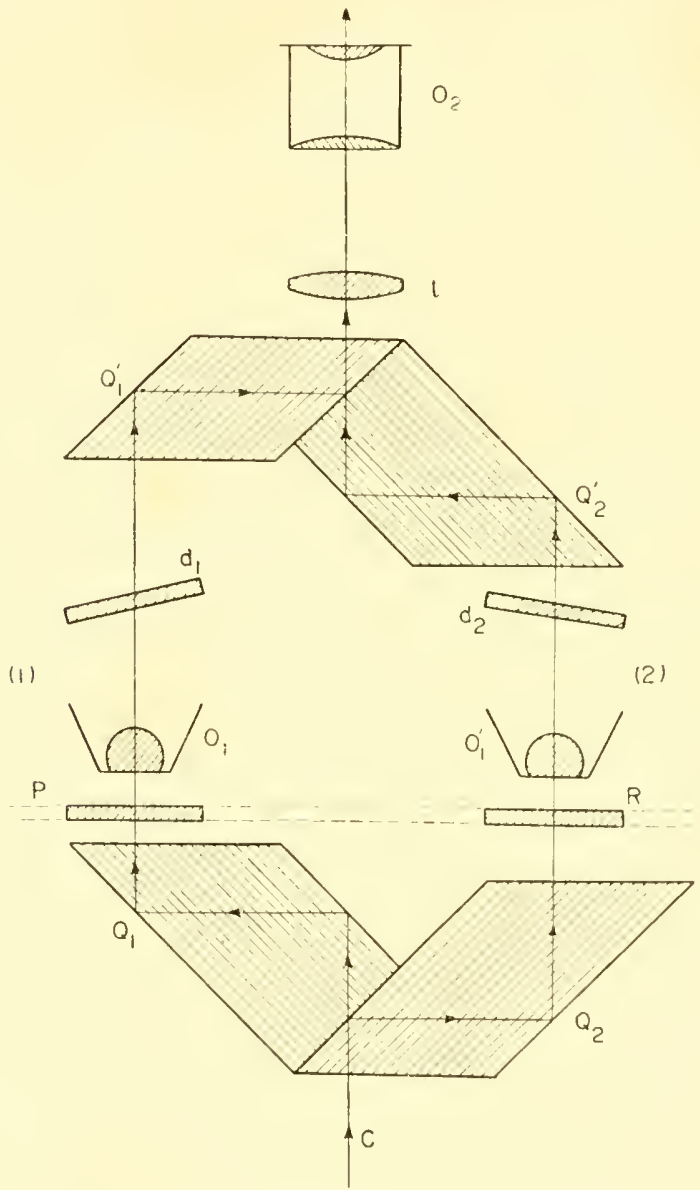

FIG. 3.8. Leilz interference microscope.

parallel-sided plates $d_{1}$ and $d_{2}$. There are, hence, lwo separate but identical microscopes: one (1) enclosing the object $P$ and the other (2) not enclosing anything (the glass slides at $P$ and $R$ are identical, too). The microscope (1) exhibits an image interfering with a reference flat wave originated by the microscope (2). No spurious image need, therefore, be expected. 


\section{POLARIZATION INTERFERENCE MICROSCOPES}

\section{Isotropic transparent objects}

In these instruments, the splitting in two of an incident ray travelling along different paths before recombining together, is effected by means of a bi-refringent system. Such instruments are two-wave interference microscopes and they only differ from the foregoing ones in the manner in which beam-splitting takes place.

The first double refraction interferometer was constructed by Jamin in 1868. It consists of the two identical plates (either quartz or spar) $L_{1}$ and $L_{2}$, cut $45^{\circ}$ from the axis (Fig. 3.9). Both plates are oriented

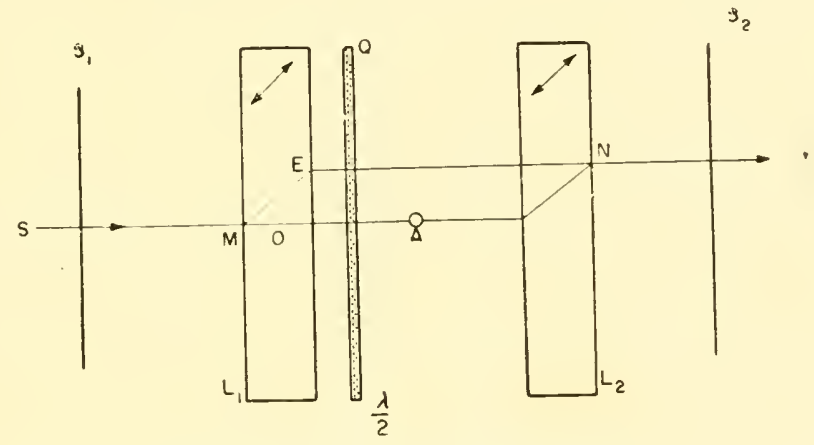

FIG. 3.9. The Jamin interferometer.

in the same direction and a half-wave plate $Q$ (the axes of which are $45^{\circ}$ from the axes of the plates $L_{1}$ and $L_{2}$ ) is inserted between them. This assembly is set between the polarizer $P_{1}$ and the analyser $P_{2}$. As it impinges against the first plate at $M$, the incident ray $S M$ is split in two thus giving rise to the ordinary ray $O$ and the extraordinary ray $E$. Owing to the half-wave plate, the vibrations of the rays $O$ and $E$ rotate $90^{\circ}$. The ray $O$ becomes extraordinary and the ray $E$ ordinary. They recombine at $N$ at the outlet of the second plate $L_{2}$. The two paths $M E N$ and $M O N$ are identical, at least for the wave-length at which the plate $Q$ is half-wave; the path difference is zero. Inserting the object at $A$ (non-birefringent transparent ordinary object) the path difference for the rays passing through $A$ is no longer zero and the object becomes visible as in the previous methods.

Lebedeff investigated the application of this interferometer to microscopy and was the first to construct a polarizing interference microscope. Figure 3.10 shows Lebedeff's microscope: it consists of 
Jamin's interferometer, inserted between the condenser $C$ and the microscope objective $O_{1}$. The polarizers are on each side of the plates at $\mathscr{L}_{1}$ and $\mathscr{L}_{2}$. The system, as devised by Lebedeff, is only suitable for wide front-lenses and. therefore of low magnification.
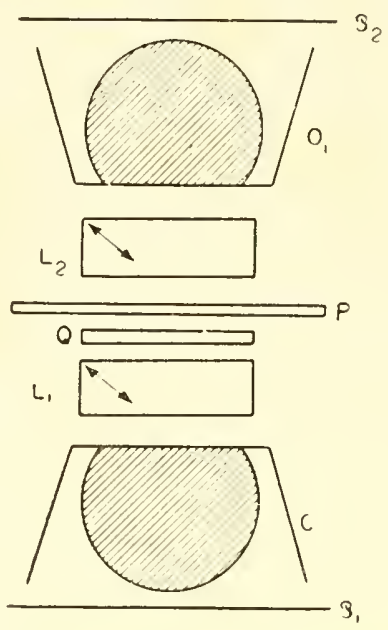

FIG. 3.10. Lebedeff interference microscope.

\section{THEORETICAL PRINCIPLE OF POLARIZING INTERFERENCE MICROSCOPES}

In all polarizing interference microscopes the image is duplicated. In Fig. 3.11, the ordinary ray originated by $S_{1}$, emerging in the direction (1) passes through the object $A$ as does the extraordinary ray
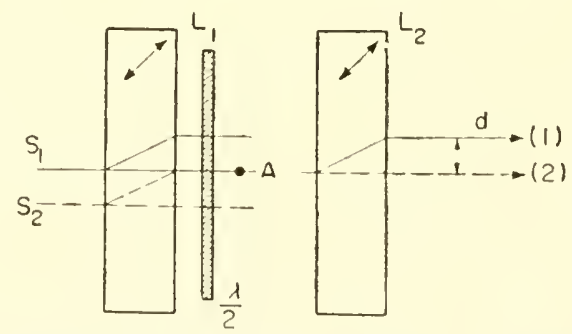

FIG. 3.11. Image duplication in a polarization interferometer.

originated by another ray $S_{2}$ emerging in the direction (2). Therefore, when looking at $A$ through the system, the two images (1) and (2) are seen, which are offset by the distance $d$. To such shift must be added another: the longitudinal one towards the rays, because the 
refraction index of the birefringent system is not the same for the ordinary and the extraordinary ray. Both images of $A$ are not seen in the same plane: they are shifted in width and in depth. This phenomenon applies to all polarizing interferometers and, in accordance with the amount of such shift, they may be classified in the following three types of instruments:

(a) Those in which the lateral inage duplication exceeds in width the object's size (shift in depth is negligible);

(b) Those in which the lateral image duplication is smaller in width than the object (shift in depth is negligible);

(c) Those in which the lengthwise image duplication is prominent and of paramount importance.

\section{Type "a" microscopes. Complete image duplication}

We will first set forth the working principle of the three above types and then describe the chief polarizing interference microscopes.

Let us consider the transparent specimen $P$ comprising at $A$ an area of optical thickness different from the remainder of the specimen (Fig. 3.12). The object $A$ may be a bacterium immersed in a liquid, an inhomogeneity within a transparent substance and so forth.

If the incident wave-surface $\Sigma$ is flat, it becomes $\Sigma$ after passing through the object, its deformations being caused by the optical-path variations within the specimen. Let us place after the specimen, the

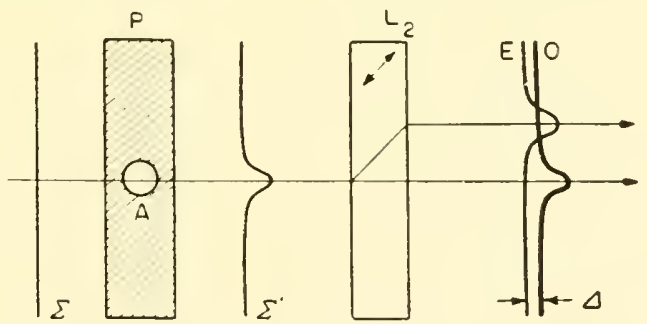

FIG. 3.12. Complete image duplication method.

birefringent plate $L_{2}$ and, as shown in Figs. 3.9 and 3.10, a polarizer and an analyser (not shown in Fig. 3.12) on both sides of $L_{2}$. Having passed through the plate $L_{2}$. the wave $\Sigma^{\prime}$ is duplicated into the waves $O$ and $E$.

It is assumed that the sidewise shift is greater than the object so that the two images of $A$ do not overlap. The depth shift $J$, assumed 
to be small, is the path difference between the waves $O$ and $E$. Both polarizer and analyser (not shown in Fig. 3.12) bring about the interference of the waves. To ensure adequate sideshift, thickness of the plate $L_{2}$ is to be rather substantial and $L_{1}$ wil. not ever be so small as to be able to use white light. In the case shown in Fig. 3.12, the experiment should be performed in monochromatic light. However, the path difference $I$ can be made as small as desired, while retaining a substantial image duplication, by a slight alteration of the device shown in Fig. 3.12. The solution is shown in Lebedeff's layout by the plate $L_{1}$ and the half-wave $Q$ (Fig. 3.10). This makes work in white light feasible. Every type of interference microscope has its own solution. We will describe in the following paragraphs the chief instruments so devised. For the time being, we are using Fig. 3.12 merely to expound the working principle and it is assumed that any value may be given to 4 . Neither are we defining more accurately the birefringent layout in relation to the microscope. What is known is that the microscope's objective images the object and in the image duplicated by the plate $L_{2}$, the two waves $O$ and $E$ are arranged as shown in Fig. 3.12. Said waves are shown at a greater scale in Fig. 3.13. Both images of the object $A$

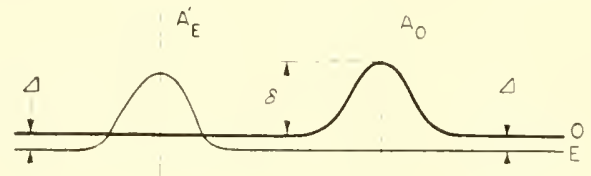

FIG. 3.13. The duplicated waves.

are at $A_{O}^{\prime}$ and $A_{E}^{\prime}$. The path difference is $1 \mathrm{in}$ the areas wherein the waves are flat and parallel, i.e. the imageless areas. At $A_{O}^{\prime}$ and $A_{E}^{\prime}$, the path difference between the waves $O$ and $E$ has not the same value. If $\partial$ is the path difference originated by the object, the path difference between the waves $O$ and $E$ will be $1+0$ at $A_{O}^{\prime}$ and $1-\partial$ at $A_{E}^{\prime}$. The light-intensity in the two images $A_{O}^{\prime}$ and $A_{E}^{\prime}$ differs from the intensity in the remainder of the field. The transparent object becomes visible.

Let us adjust the instrument so that I equates the $0.565 \mu$ wavelength and let us assume that both polarizer and analyser are crossed. In accordance with the classical data pertaining to polarized-light interference, the first-order purple hue (Newton's scale of colours, Chapter VII, $\$ 1)$ is observed in the image-less areas. At $A_{O}^{\prime}$, the path 
difference is $\delta+0.565 \mu$ and, at $A_{E}^{\prime}, \delta-0.565 \mu$. The colours are no longer the same. Let us take, for instance $\delta=0.015 \mu$. The path difference is $0.580 \mu$ at $A_{O}^{\prime}$ and $0.550 \mu$ at $A_{E}^{\prime}$. According to Newton's scale of colours, the hue is indigo blue at $A_{O}^{\prime}$ and dark red at $A_{E}^{\prime}$. Hues are symmetrical in relation to the purple. Owing to the structure of the phase-shifting object it follows that the colours are not the same at every point of the images $A_{O}^{\prime}$ and $A_{E}^{\prime}$. The hues exhibit the optical-path variations within the object and a cursory survey determines accurately the phase variations.

Altering the adjustment instrument will give $1=0$. The whole field is dark, save in the areas $A_{O}^{\prime}$ and $A_{E}^{\prime}$ which are bright. The dark ground is the conventional one with the difference that the areas $A_{O}^{\prime}$ and $A_{E}^{\prime}$ are coloured to a greater or lesser extent according to the phase variations they give rise to, the colour showing directly the phase-shift. Let us adjust the instrument so that 1 be very small, $0.04 \mu$ for instance: the field becomes dark grey. The image is similar to high-absorption phase-contrast. Here again, provided the objectoriginated phase variations be adequate. they appear under the form of colours. Otherwise, only variations in intensity are perceived.

Increasing the phase-shift between the waves $O$ and $E$, i.e. increasing 4 . gives rise to coloured contrasts and, when $\lrcorner=0.565 \mu$, the purple colour is again visible. At higher 1 values. contrast is not so good and sensitiveness drops.

The best performance is achieved when $J$ is very small and the ground dark grey but then measurements are not so easily carried out. The purple hue gives perhaps slightly less contrasted images but, conversely, as shown later, measurements can be made easily. The complete-image-duplication method just described is very sensitive and very suitable for not too large objects: minute discrete details can thus be observed under good conditions. As very large image duplications are not feasible, the method is not applicable to very wide objects as, then, the two images overlap.

\section{Type " $b "$ microscopes. Differential method}

The following method applies to any object but is less sensitive. Let us consider anew Fig. 3.12 and select a much thinner plate $L_{2}$ so that the lateral shift be small in relation to the object's width. In the object's image $A^{\prime}$, the waves $O$ and $E$ are arranged as shown in Fig. 3.14. In imageless areas, the path difference between the two waves equates $\Delta$. In the area of the image $A^{\prime}$ itself the path difference 
is no longer 1 . At (1) and (2) the path difference has a value depending on the lateral shift and the slope of the wave surfaces. Intensity at (1) and (2) differs from the remainder of the field surrounding the image. The object is seen, therefore, according to its "slopes"; it is the opticalpath gradients, e.g. the index gradients, which are evidenced. The

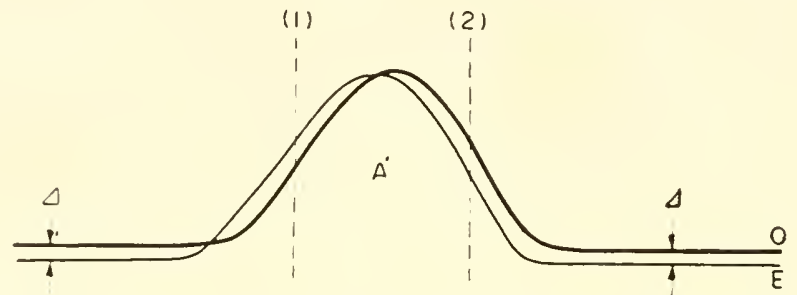

FIG. 3.14. The duplicated waves in the differential method.

method is differential. Figures 3.15 (a) and (b), showing outlined wave surfaces, will help to clarify these phenomena. These two figures reproduce the left section of Fig. 3.14 merely eliminating the rounded-off contours. In Fig. 3.15(a), the path difference $I$ between the two waves $O$ and $E$ is zero. Owing to the lateral shift $d$ between $O$ and $E$ and the slope $a$ of the wave surface, it is readily seen that the path difference between the two waves $O$ and $E$ in the area (1) equates $\alpha \cdot d$ (assuming $\alpha$

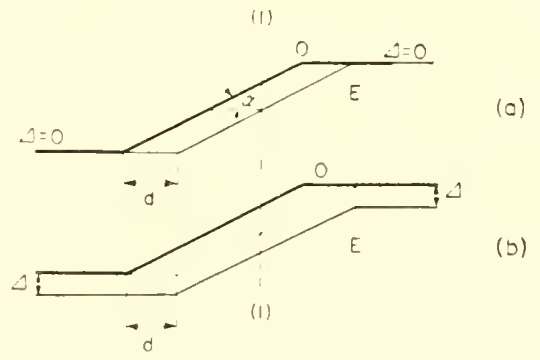

FIG. 3.15. Path difference between the two waves $O$ and $E$.

to be not steep). If, as in Fig. 3.15(b), the path difference 1 between the two waves $O$ and $E$ is not zero, the path difference in the area (1) becomes $1-u d$. In any case, when changing over from an inageless area to one where the object is featured by the surface-wave slope $u$, the path difference steadily varies by $a . d$.

If $\angle=0.565 \mu$, the field is purple outside the image (crossed polarizers). The hue varies in the $\iota$-slope area (1): when $a d=0.015 \mu$ 
the colour is indigo. As previously, sensitivity is improved by giving to 1 a low value, e.g. $0.04 \mu$, that gives rise to the dark grey field. When $\Delta=0$, the image is on a dark ground type but it is the object's slopes that are detected. Lebedeff has described the full-image duplication and the differential processes. In the latter he employed several birefringent systems so as to match image duplication with every type of object thus achieving, in all cases, utmost sensitiveness. However, the instrument was not convenient. We were able to generalize the differential process by stating that microscopic specimens exhibit usually rather marked phase-shifting properties. We then suggested to select, once and for all, an image duplication value close to the instrument resolving power. Image duplication is virtually invisible and any object may be examined by the same instrument. As will be seen later, it is then possible to devise highly convenient interference microscopes.

\section{Type "c" axial image duplication microscopes}

In these instruments, the ordinary image $O$ is far removed from the extraordinary image $E$ (Fig. 3.16). When the ordinary image $O$ is focused by the eyepiece $O_{2}$, owing to faulty focusing, the image $E$ vanishes. There is, of course, some extraordinary light $E^{\prime}$ in the plane

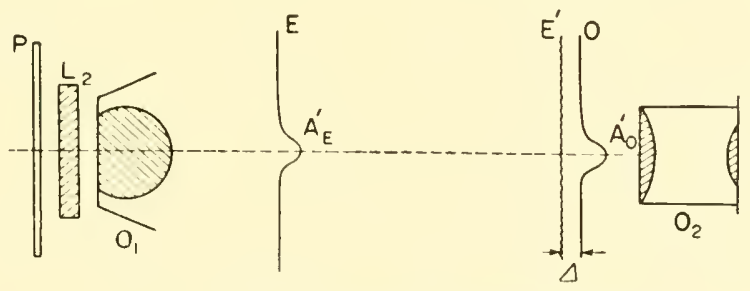

FIG. 3.16. Axial image duplication method.

of the ordinary image. It is well known, too, that slightly defective focusing in microscopy causes the image to vanish while a uniform field emerges. This is what now happens to the extraordinary image. The extraordinary light $E^{\prime}$, in the plane of the ordinary image, gives rise to a virtually uniform field: interference is taking place between the ordinary wave and the uniform ground $E^{\prime}$ originated by the nonfocused extraordinary wave. The transparent object is examined without the objectionable duplication. However, this process is not so sensitive as the full image duplication method. As in all other processes, the path difference $I$ between the ordinary wave $O$ and 
the ground $E^{\prime}$ is adjustable. In Fig. 3.16, the ordinary and extraordinary images are both duplicated axially only although, in some instruments such axial duplication is concomitant with lateral some shift that does not, however, affect the principle of the process.

\section{SMITH'S FULL IMAGE DUPLICATION INTERFERENCE MICROSCOPE}

This type of microscope is based on the use of Wollaston prisms. It is well known that the latter consist of two quartz prisms the axes of which are crossed (Fig. 3.17). One axis lies in the plane of said

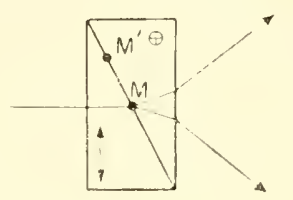

FIG. 3.17. Wollaston prism.

figure, the other is perpendicular to it. An incident light-ray, normal at the ingoing face, is split, at $M$, in two rays: the ordinary ray $O$ and the extraordinary ray $E$. Phenomena occur as in Figs. 3.9 and 3.12 but the linear duplication is replaced by an angular one. If the point $M$ lies in the area where both prisms have the same thickness, the outgoing rays are in phase. If the incident ray impinges the prism at $M^{\prime}$ the outgoing rays $O$ and $E$ are no longer in phase. Therefore. the phase difference between the rays $O$ and $E$ may be varied according to the Wollaston area used. Observing a Wollaston prism between two polarizers there is a whole series of fringes that are parallel, equidistant and straight in relation to the Wollaston prism edges. In white light and between crossed polarizers there is a dark fringe edged on both sides by colour fringes. The colour sequence is substantially that in Newton's scale of colours. The dark fringe is at $M$ in the area where both prisms have the same thickness. Now let us image a narrow source at $M$ on the dark fringe (Fig. 3.18) and let us assume the beam's aperture angle a to be narrow. The rays $O$ are in phase with the ralys $E$ (such would not be the case were the angle a to exceed at few degrees). Since both polarizers $\mathscr{L}_{1}$ and $\mathscr{L}_{2}$ are crossed. no trace of the beam on the screen $T$ remains. In Fig. 3.18, duplication of the emerging beam is not shown: it is assumed that such duplication is minimal in relation to the beam width. Let us move the Wollaston prism at right angles to the mean ray of the incident beam, e.g. in 
direction of the arrow. The incident beam ends in the prism at a point above $M$ : the outgoing rays $O$ and $E$ are no longer in phase.

Let us assume that the source is imaged on the first-order purple fringe within the prism: the outgoing rays $O$ and $E$ are in phase in the yellow only. The beam's trace on the screen $T$ is purple.

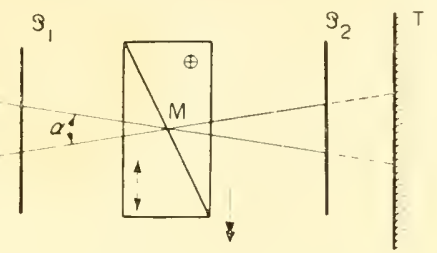

FIG. 3.18. Use of the Wollaston prism.

These preliminary considerations will help in comprehending the principle of Smith's interference microscope, shown diagrammatically in Fig. 3.19. The specimen is at $P$; the wave surface $\Sigma$ is deformed after passing through the transparent object. Owing to the Wollaston prism $W$. located next after the objective $O_{1}$, one finds the waves $\Sigma_{1}$ and $\Sigma_{2}$ in the image plane and arranged as are the waves $O$ and $E$ in Fig. 3.13. Owing to the waves passing through the Wollaston prism's two elements. the ordinary wave becomes extraordinary and vice versa: there is no need. therefore. to denote them otherwise than by numerals. A polarizer should be placed ahead of and an analyser next after $W$ (neither are shown in Fig. 3.19). Shifting sidewise the prism $W$ (as

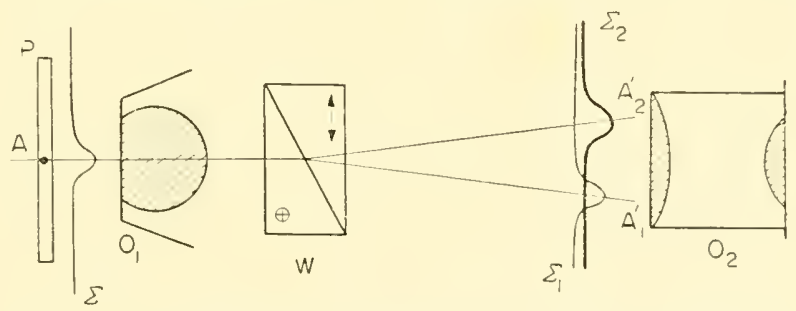

FIG. 3.19. Principle of the Smith interference microscope.

in Fig. 3.18) or using a compensator will evince the object image at $A_{1}^{\prime}$ and $A_{2}^{\prime}$ in many colours. In order to have the images $A_{1}^{\prime}$ and $A_{2}^{\prime}$ surrounded by a uniform field the light-beam passing through $W$ must be very narrow, whereupon it goes through a definite fringe within $W$ and the uniform field is secured. If, for instance, the beam 
passes through $W$ in the dark-fringe area, it should not overlap this fringe so that the field surrounding $A_{1}^{\prime}$ and $A_{2}^{\prime}$ may remain very dark. A narrow slit, located in the condenser focus (not shown in Fig. 3.19) should therefore complement the microscope diagram (same figure). The specimen $P$ is parallel-beam illuminated and the narrow slit imaged on a fringe within $W$. The latter therefore must lie in the objective's focal point. To dispense with the slit, i.e. prevent substantial closing of the condenser, Smith adds a second Wollaston prism to the instrument's condenser. Figure 3.20 shows the final layout of Smith's

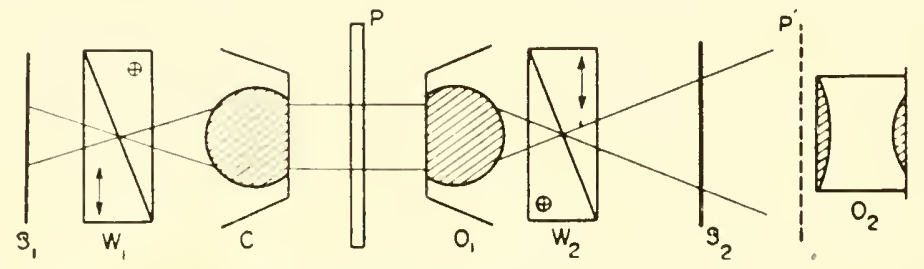

FIG. 3.20. Smith interference microscope.

microscope constituent elements. The optical elements located to the right of $P$ (Fig. 3.20) are identical with those shown in Fig. 3.19. The additional birefringent element is the Wollaston prism $W_{1}$, located in the focal plane of the condenser $C$ and associated with $W_{2}$ through the condenser $C$ and the objective $O_{1}$. The polarizer is ahead of $W_{1}$ and the polarizer next after $W_{2}$. Other birefringent elements may be set between $W_{2}$ and $\mathscr{L}_{2}$ as required. The $W_{1}$ fringes are then exactly superimposed on those of $W_{2}$ and both prisms are adjusted so that their mutual birefringence is cancelled out. Under such conditions, there is no need to diaphragm the condenser save for stray light. If the focal length of the condenser $C$ and the objective $O_{1}$ are equal, the previous requirement is met by using two identical Wollaston prisms. If the focal length of $C$ or $O_{1}$ differ, suitable values are selected for the prism angles so that the inner fringes of the two prisms be superimposed at all times.

Every objective has its own Wollaston prism and the condenser"s $W_{1}$ prism is changed concurrently with the objective.

If the objective $O_{1}$ is of low-power, its focal plane is accessible and the $W_{2}$ prism readily set at the focal plane.

If the objective $O_{1}$ is of high-power, the $W_{2}$ prism is inserted between the elements of the specially designed objective. Figure 3.21 shows Smith's microscope as constructed by Messrs. C. Baker Ltd. 
Dyson has devised a Lebedeff-type interference microscope. To secure substantial image duplication, thick plates are required: this requirement is not consistent with powerful objectives. To obviate this

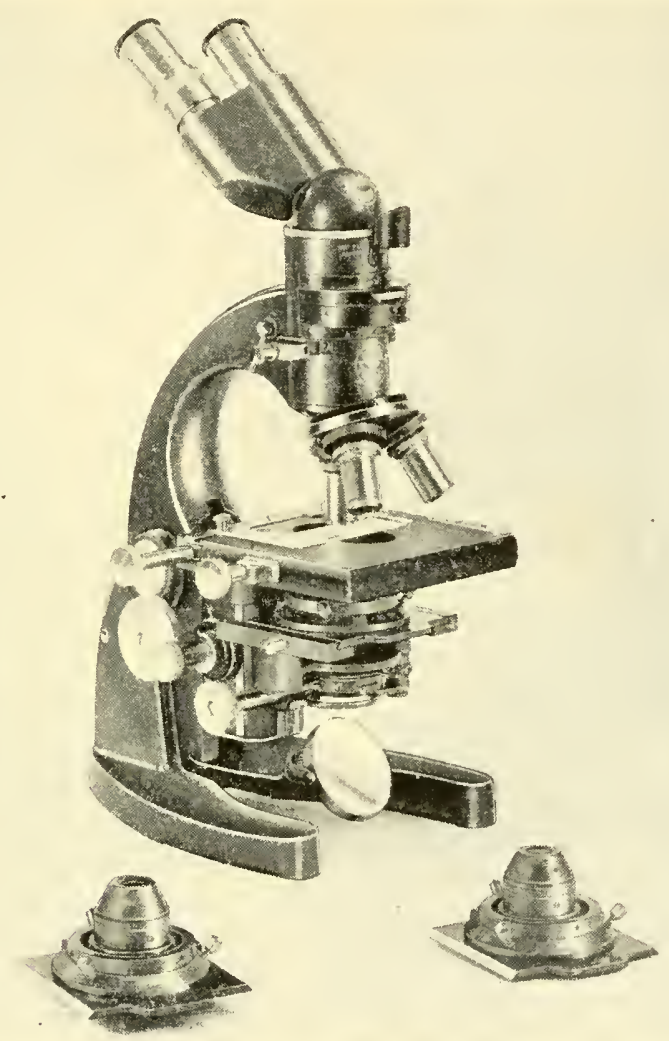

FiG. 3.21. Smith interference microscope (C. Baker).

stumbling block, Dyson provides a half-sphere whose lower part consists of the two interferometer plates. Owing to the half-sphere, thick plates are between the specimen and the objective: the microscope shows an interferential image.

\section{FRANÇON'S COMPENSATED INTERFERENCE EYEPIECE}

The foregoing differential method is applied to the interference eyepiece whose basic birefringent element is Savart's polariscope consisting of two identical quartz plates cut $45^{\circ}$ from the axis and crossed 
(Fig. 3.22). The axis of the second plate does not lie in the plane of Fig. 3.22. In fact, the axis is $45^{\circ}$ from the plane of Fig. 3.22.

In the first plate, the incident ray splits in two: the ordinary ray $O_{1}$ and the extraordinary ray $E_{1}$. As the second plate is $90^{\circ}$ from the first. the ordinary ray of the latter becomes extraordinary in the second and vice versa. The dotted line shows that the ray $O_{1} E_{2}$, emerging

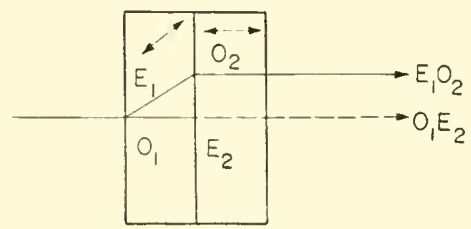

FIG. 3.22. Savart plate.

from the second plate, is not in the plane of the figure but parallel to the ray $E_{1} O_{2}$ which does lie in said plane. Figures 3.23(a) and (b) readily show the rays' paths. Figure 3.23 (a) shows the rays' trace in a front plane after passing through the first plate. The ordinary and extraordinary rays are at $O_{1}$ and $E_{1}$, respectively. Figure 3.23(b) shows the rays" path in a front plane after passing through the second plate. The ray $O_{2}$ extends the ray $E_{1}$ but the ray $E_{2}$ is shifted horizontally.

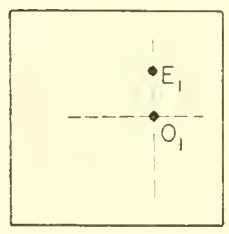

(a)

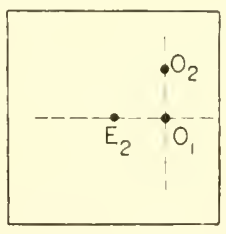

(b)

FIG. 3.23. Ray paths in the Savart plate.

In the horizontal plane. the $E_{2}$ shift in relation to $O_{1}$ is the same as that of $E_{1}$ in relation to $O_{1}$ in the vertical plane. When the incident ray is normal in relation to Savart's polariscope the outgoing rays $E_{1} O_{2}$ and $O_{1} E_{2}$ are in phase. Slightly tilting the polariscope varies the path difference which is thus readily adjustable. Let us place Savart's polariscope between two polarizers and observe through this system a remote uniformly-illuminated object, such as the sky. If the eye is not accommodated, a series of virtually straight, parallel and equidistant fringes are perceived. If the polarizers are crossed, there are, on each side of the dark central fringe, colour fringes which are 
similar to those observed in the Wollaston prism. The difference is in the location of such fringes. In the case of the Wollaston prism, they are perceived within the latter as if they were painted. Savart's polariscope requires on-infinity observation. Changing the polariscope direction in relation to the eye, the fringes move as a whole in accordance with the motion imparted to the instrument.

These preliminary notes are intended to make clear the working principle of the interference eyepiece. Referring to Fig. 3.24, Savart's polariscope $S$ is placed between the field lens $O_{2}$ and the eyepiece

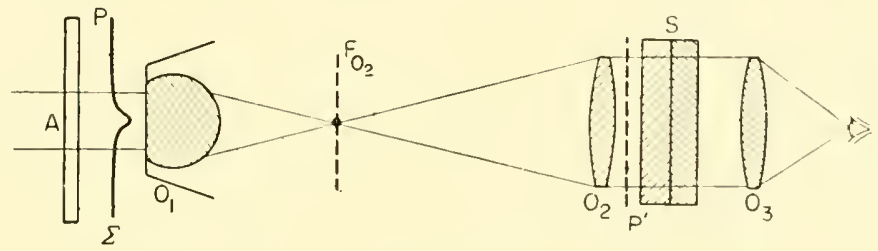

FIG. 3.24. Principle of the interference eyepiece.

lens $O_{3}$. Neither the polarizer ahead of $S$ nor the analyser next after it are shown in Fig. 3.24. The specimen is at $P$ and the wave surface $\Sigma$ is deformed after passing through the transparent object. The objective $O_{1}$ images the specimen $\left(P^{\prime}\right)$ close to the field lens $O_{2}$. The Savart polariscope $S$ splits the wave incoming at $P^{\prime}$ in two waves whose arrangement is that shown in Fig. 3.14. As explained in $\$ 5$. the transparent object is perceived by its slopes. In order to have the image surrounded by a uniform field, as was the case with the Wollaston prism, the illuminating beam must be formed on a fringe of the Savart polariscope. We have already seen that these fringes are on infinity; they should therefore be found, in Fig. 3.24, at the image focal-point $F_{\mathrm{O}_{2}}$ of the objective (in which case they are virtual). They could actually be observed at $F_{\mathrm{O}_{2}}$ provided the direction of light were altered, e.g. substituting the source for the eye.

Hence, to have a uniform field, a narrow slit must be placed in the condenser focal-plane (not shown in Fig. 3.24). The specimen $P$ is parallel-beam illuminated and the narrow slit imaged on one of the Savart polariscope fringes at $F_{O_{2}}$. Slightly tilting the polariscope the fringes are shown seriatim at $F_{\mathrm{O}_{2}}$ : the path difference is varied and the image seen in many colous. To obviate using a slit and allowing the condenser to have a normal aperture, a compensating device is provided ahead of the condenser. Figure 3.25 shows the final layout 
of the compensated interference eyepiece. The optical elements to the right of $P$ in said figure are identical with those in Fig. 3.24. The additional element brought in is the Savart polariscope, placed in any position ahead of the condenser $C$. The on-infinity fringes of the polariscope are beamed in the condenser focal-plane $F_{C}$, plane which is always ahead of the specimen $P$.

The objective $O_{1}$ images the plane $F_{C}$ at $F_{O_{2}}$ and, owing to the short focal length of $O_{1}$, the plane $F_{O_{2}}$ is not far from the objective's focal plane. The fringes of the polariscope $S_{1}$ are seen at $F_{O_{2}}$. Provided
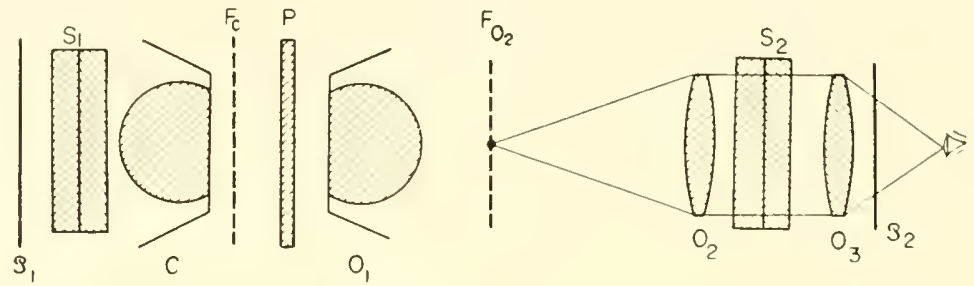

FIG. 3.25. Principle of the compensated interference eyepiece.

the focal length of the lens $O_{2}$ is suitable, the plane $F_{O_{2}}$ can be made to match the focal plane of the eyepiece lens $O_{2}$ whereupon the $S_{1}$ fringes and the $S_{2}$ virtual fringes are in the same plane. Provided the spacing of the fringes, originated from $S_{1}$ and $S_{2}$, be the same and the two polariscopes in the proper direction, then birefringences cancel out.

There is no longer need to diaphragm the condenser which may be used with its standard diaphragm.

As in all polarizing devices, the polarizer $\mathscr{L}_{1}$ is ahead of $S_{1}$ and the analyser $\mathscr{L}_{2}$ next after $S_{2}$.

Let us change the objective $O_{1}$ : if the focal length is different, so is the position and spacing of the fringes of $S_{1}$. Theoretically, the polariscope $S_{1}$ should be changed, too. In fact, the solution is much simpler: merely setting the condenser nearer to or farther from the specimen will serve the purpose. This motion alters the magnification between the planes $F_{C}$ and $F_{O_{2}}$ and. hence, the fringe spacing shown by $S_{1}$ at $F_{\mathrm{O}_{2}}$. Compensation is well correlated. In the latter adjustment, the Köhler illumination is diverged from owing to the condenser molion. To obviate this difficulty, the mechanical member securing $S_{1}$ to the condenser comprises a small revolving plate fitted with two divergent lenses and a free aperture. Rotating the plate 
brings under the condenser the lens affording the Köhler illumination. The divergent lenses are used in dry-front objectives and the free aperture in oil-immersion objectives employed under normal conditions. The instrument then comprises the two independent units shown
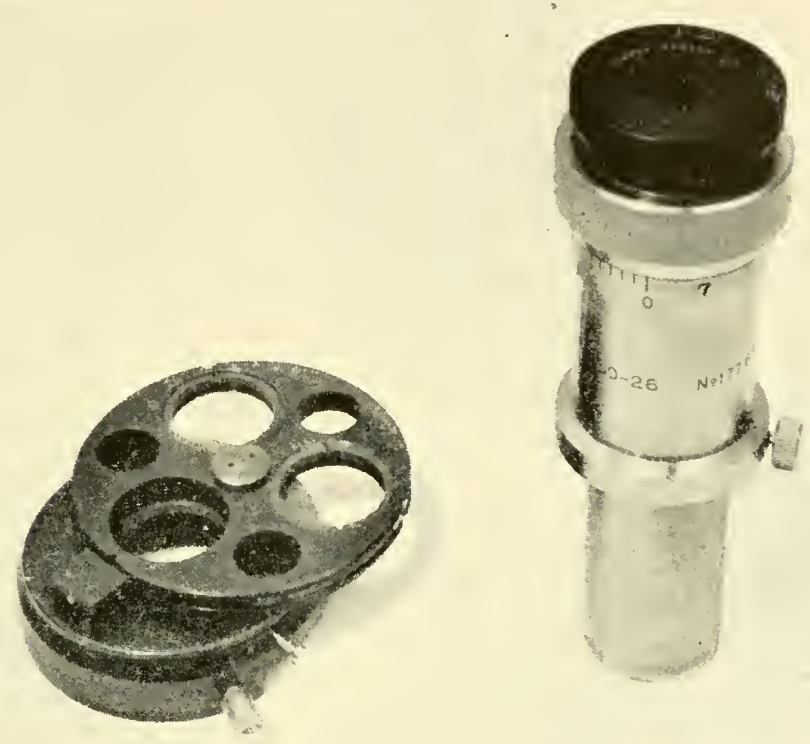

FIG. 3.26. Françon's compensated interference eyepiece (Barbier, Benard, Turenne).

in Fig. 3.26: the interference eyepiece (unit $O_{2}, S_{2}, O_{3}, \mathscr{L}_{2}$ ), substituted for the standard one and the compensator (unit $\mathscr{L}_{1}, S_{1}$ ). secured under the condenser. Owing to the versatility of the compensating system it may be fitted to almost all types of microscopes.

\section{L. P. JOHANSSON'S INTERFEROMETER EYEPIECE}

This instrument makes use of the full image duplication process with a Savart plate and a condenser illuminating-slit. A glass plate, whose sides are not quite parallel, enables one to make measurements. The eyepiece layout diagram is shown in Fig. 3.27. A prism $Q$ (ordi- 
nary glass) is inserted between the eyepiece elements $O_{2}$ and $O_{3}$ ahead of the Savart polariscope $S$.

The polaroid members are not shown in Fig. 3.27. A small aperture is provided in the centre of the wedge $Q$. Rotating the latter about the microscope centre-line alters the direction of the beam traversing

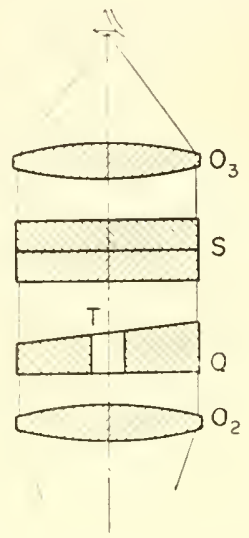

FIG. 3.27. L. P. Johansson device (Jungnbolaget).

the wedge. This is tantamount to moving the imaged slit in relation to the on-infinity fringes of the Savart polariscope. Hence, the path difference 1 between the two waves (Fig. 3.13) can be varied ad libitum. However, the light passing through $T$ remains unaltered and serves as reference. In this way measurements may be made by comparing the imaged-object colours with the reference area.

\section{NOMARSKI'S INTERFERENCE MICROSCOPE}

Nomarski's microscope likewise makes use of the differential method. It differs from Smith's microscope in an alteration of the next-after-the-objective Wollaston prism. In connection with Smith's microscope, we mentioned that both $W_{1}$ and $W_{2}$ are associated (Fig. 3.20) in order to exhibit a uniform field in the image plane. When $W_{2}$ is, for instance, slightly to the right of its usual position, the image field is streaked by colour fringes. As Smith observed, a uniform field may be reverted to by setting, in the image plane, a Wollaston-lype compensator whose own fringes are spaced alike thus cancelling out the former ones. Such an additional member may be obviated by designing a special objective wherein $W_{2}$ is in the focal 
plane. In Nomarski's interference microscope this problem is solved by substituting the next-afrer-the-objective Wollaston prism by an altered prism of $W_{2}$-type. The latter is no longer cut parallel to the axis thus conducing to a different fringe arrangement. In the usual Wollaston prism, the fringes are localized within it in the plane $F$

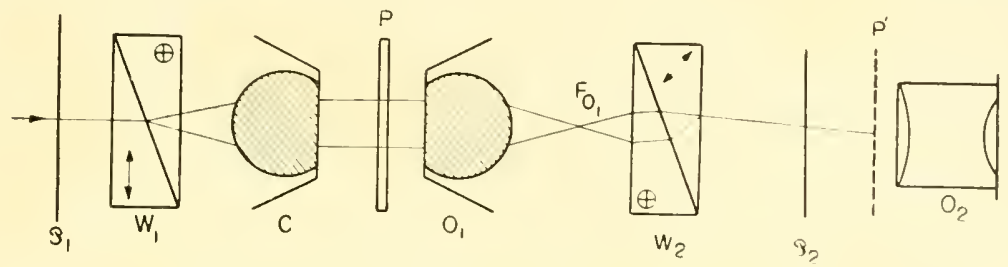

FIG. 3.28. Nomarski interference microscope.

(assuming the angle of both prisms is small) (Fig. 3.29) whereas, in Nomarski's prism, such plane is outside it (Fig. 3.30). Now, this plane can be made to coincide with the objective's inaccessible plane, as shown in Fig. 3.28. If its fringes are to overspread the imaged fringes of $W_{1}$, a standard Wollaston prism should be at $F_{O_{1}}$. Owing to the former alteration, $W_{2}$ can be set more to the right, viz. outside the objective but with its fringes at $F_{O_{1}}$. As soon as the localizing

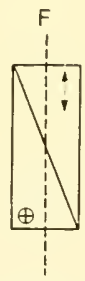

FIG. 3.29. Localization of fringes in a Wollaston prism.

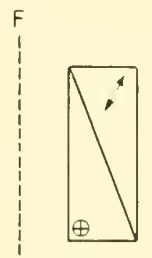

FIG. 3.30. Localization of fringes in a modified Wollaston prism.

plane of the fringes diverges from its normal position the fringes are no longer straight. If said plane diverges too much from the prism, the fringes in-curve appreciably and compensation with $W_{1}$ is no longer perfect. Therefore the axis slope of $W_{2}$ is to be given the value required so that its fringes coincide with the objective's focal point and $W_{2}$ remains just outside the objective. The appearance of images is identical with that described in $\$ \S 5$ (differential method) and 7. 


\section{AXIAL IMAGE DUPLICATION INTERFERENCE MICROSCOPES}

Smith investigated an instrument, based on microscopes of the type c, described in $\S 5$, which was built by Messrs. C. Baker Ltd. This instrument is shown in Fig. 3.21: it is convertible and either the full sidewise-duplication or the method now described can be applied to it.

Reference to Fig. 3.31 shows the spar plate $Q_{2}$, only a few tenths of a millimeter thick, cemented against the frontal lens of the mic-
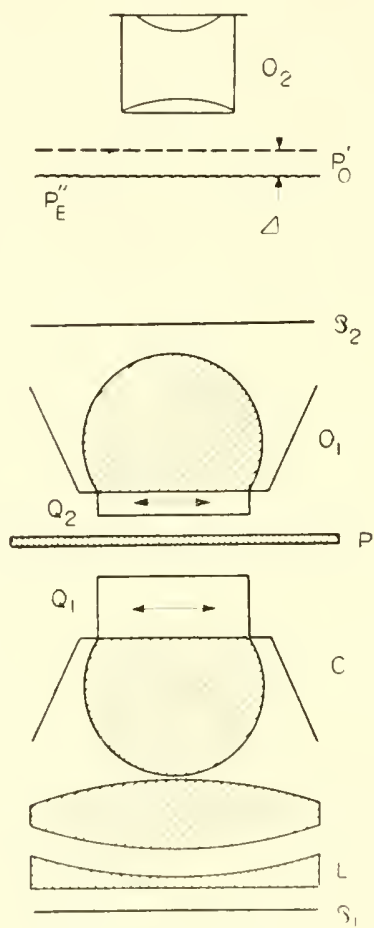

FIG. 3.31. Smith interference microscope.

roscope objective $O_{1}$ and cut parallel to its axis. It is well known that the surface-wave of a single-axis crystal such as spar comprises two portions: the ordinary and the exiraordinary wave surfaces (a sphere and an ellipsoid, respectively). When a beam of ordinary rays passes through the objective $O_{1}$. the latter images the specimen $P$ at $P_{O}^{\prime}$. The effect on these rays is that obtaining had a mere glass plate been cemented to the front lens. The effect is quite different 
on the extraordinary rays. The extraordinary wave is an ellipsoid of revolution about the horizontal axis of the spar plate $Q_{2}$. Owing to the curvature changes of the extraordinary waves, the extraordinary image exhibits considerable astigmatism: there is virtually no extraordinary image left. Nevertheless, as stated in $\$ 5$, the extraordinary light provides the uniform ground $P_{E}^{\prime \prime}$ in the plane of the ordinary image $P_{O}^{\prime}$. The transparent details of the specimen are perceived owing to the interference taking place between $P_{O}^{\prime}$ and the uniform ground $P_{E}^{\prime \prime}$. The path difference $\triangle$ between $P_{O}^{\prime}$ and $P_{E}^{\prime \prime}$ may be varied and various colour contrasts observed by means of a compensator, not shown in Fig. 3.31.

If the instrument comprised only the spar plate $Q_{2}$, diaphragming the condenser would be required. To dispense with this, the quartz plate $Q$, cut parallel to the axis, is set on the condenser $C$. Provided the thickness of the plates $Q_{1}$ and $Q_{2}$ have a suitable ratio, the unit exhibits on-infinity circular fringes lying in the object focal-plane of the condenser $C$. To eliminate them and have a uniform pupil, a birefringent element, exhibiting circular fringes localized in its own plane, is set in the focal plane of the condenser $C$. The two fringe systems cancel out and diaphragming is no longer required.

Several axial image duplication instruments have been devised. In Blaisse's interference microscope, the plate $Q_{2}$ is a spar plate. The plate $Q_{1}$ is of rutile. Both plates are cut at right angles to the axis.

Françon's interference microscope comprises a combination of a half-sphere and two quartz parallel-side plates whose axes are sloping (Fig. 3.32). Employing the half-spheres described previously, thick quartz plates may be substituted for spar. Both plates $Q_{1}$ and $Q_{2}$ are identical and cut slantwise in relation to the axis. The plate $Q_{2}$ is cemented to the quartz plano-convex lens $Q_{3}$, cut parallel to the axis. The elements $Q_{2}$ and $Q_{3}$ make up the half-sphere. The analyser $\mathscr{L}_{2}$ is cemented between $Q_{2}$ and $Q_{3}$. The latter is set so that its axis is at right angles to the axis of $\mathscr{L}_{2}$. Under such conditions, the lens $Q_{3}$ exhibits no birefringence and takes no part in the interference process. Provided the vibration passing through $\mathscr{P}_{2}$ is directed at right angles to the axis of $Q_{3}$, the latter's role is merely to complement the halfsphere with glass of the same index as $Q_{2}$.

The plate $Q_{2}$ gives rise to the axial duplication which is now concomitant with a sidewise duplication. $A^{\prime}$ is an ordinary image of $A$, this interferes with the uniform extraordinary light: the image $A^{\prime}$ can 
now be readily observed through the objective $O_{1}$ of an ordinary microscope.

The plate $Q_{1}$, identical with $Q_{2}$, is cemented against the condenser which dispenses with diaphragming.

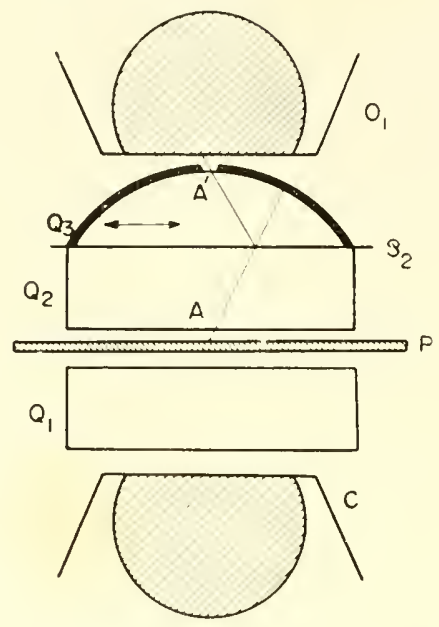

FIG. 3.32. Françon interference microscope.

Cementing a half-wave plate to $Q_{1}$, the system $Q_{1} Q_{2}$ becomes a Jamin interferometer.

Altering the former layout by rotating the plate $Q_{1} 90^{\circ}$ about its axis, the on-infinity fringes developed are now perfectly parallel straight lines. These fringes can be eliminated, and contrasts varied, by means of a Wollaston prism set in the focal plane of the condenser $C$.

\section{MULTI-WAVE INTERFERENCE MICROSCOPES}

Let us consider (Fig. 3.33) the glass plates $L_{1}$ and $L_{2}$ whose faces opposite $x_{1}^{\prime}$ and $x_{2} x_{2}^{\prime}$ are semi-reflecting and parallel. The monochromatic light-ray $S_{1} I_{1}$, incoming on the plate $L_{2}$ at $J_{1}$, splits in two rays: one transmitted, $T_{1}$, and onc reflected. The latter reverts to the first plate $L_{1}$, is reflected, reverts on to $L_{2}$ and splits again in two rays, one transmitted, $T_{2}$, and one reflected. The same phenomena occur for the other ray and, ultimatcly, a large number of transmitted rays: $T_{1}, T_{2}, T_{3}$, etc. whose intensity naturally decreases as successive reflections occur. If incidence is close to normal, the rays $T_{1}, T_{2}, T_{3}, \ldots$ can be much more closely packed than Fig. 3.33 shows. Provided there is no phase object between the plates $L_{1}$ and $L_{2}$ and that $x_{1} \cdot x_{1}^{\prime}$, 
$x_{2} x_{2}^{\prime}$ be quite parallel, the same phenomenon occurs at all points of the plate. The path travelled by $T_{2}$ is longer than that of $T_{1}$; the difference is $2 e(n=1)$ assuming $e$ to be the interferometer thickness (distance from $x_{1}, x_{1}^{\prime}$ to $x_{2}, x_{2}^{\prime}$ ). Likewise, the path travelled by $T_{3}$ is longer than that of $T_{2}$, the difference still being $2 e$. Hence, every ray travels along a path the difference of which is $2 e$ in relation to the previous ray. All the $T_{1}, T_{2}, T_{3}, \ldots$ rays interfere and the light-intensity through the plate is a function of the interferometer thickness $e$ and of the wave length $\lambda$ of the light used. Provided the same source (steady $\lambda$ ) is

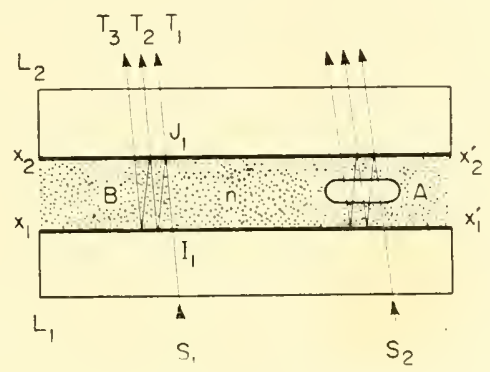

FIG. 3.33. Multi-wave interferences.

retained and $e$ be varied, the light-intensity transmitted conforms to the law shown in Fig. 3.34 in which the ordinates denote the interferometer-transmitted light-intensities as a function of the optical thickness ne ( $n$ being the index of the medium between $x_{1} x_{1}^{\prime}$ and $x_{2} \cdot x_{2}^{\prime}$ ). If the thickness $n e$ is equivalent to $\lambda, 2 \lambda, 3 \lambda$, etc., maximum light is obtained whereas, as soon as these values are diverged from, intensity drops rapidly. The more reflective are the faces $x_{1} x_{1}^{\prime}$ and $x_{2} x_{2}^{\prime}$, the sharper the peaks shown in Fig. 3.34. But, then, absorption of the device increases, thus requiring rather intense monochromatic sources, not readily obtainable. However, a satisfactory performance is evinced by evaporating multiple layers on $x_{1}, x_{1}^{\prime}$ and $x_{2} x_{2}^{\prime}$. Figure 3.34 shows that, to obtain maximum light, the thickness must be accurately determined as the slightest variation of $e$ entails at once a considerable intensity drop. This is the very fact made use of to evince transparent objects. Let us consider the transparent object $A$, sandwiched between the interferometer plates (Fig. 3.33). The multiple rays passing through the object $A$ do not follow the same path as those passing in the random area $B$ close to said object. Phenomena occur in the area $A$ as if the spacing of the interferometer plates were altered. The lightintensity varies at once, thus disclosing the presence of the object $A$. 
Let us now investigate the phenomenon in the curve shown in Fig. 3.34. Let us assume that the optical thickness of the interferometer equates $n_{1} e_{1}$ outside the object. The transmitted intensity is $I_{1}$. The optical thickness is slightly altered for the light passing through the object and becomes $n_{2} e_{2}$, giving rise to the intensity $I_{2}$, different from $I_{1}$. The transparent object becomes visible. It follows that to the same variation $n_{1} e_{1}-n_{2} e_{2}$ of the optical path is correlated an intensity variation which is the more pronounced as the curve slope increases.

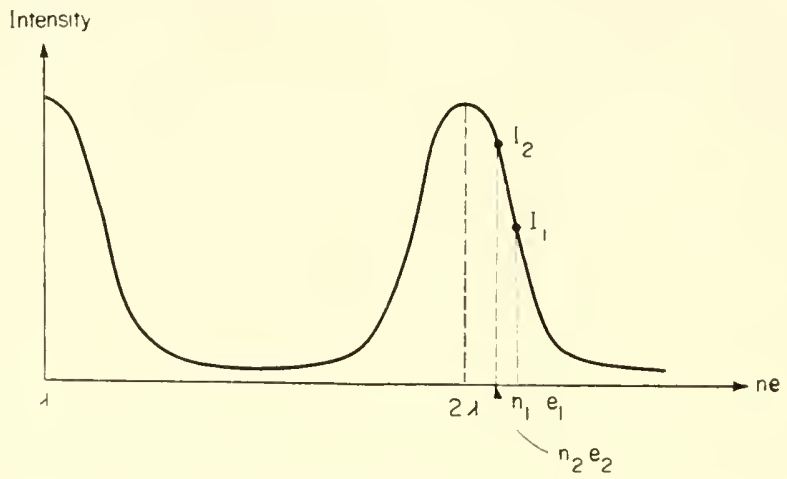

FIG. 3.34. Transmitted light intensities as a function of the optical thickness ne in multi-wave interferences.

If the object exhibits thickness or index changes, these are shown as light-intensity changes that enable one to thoroughly investigate the object's structure. The sharper the curve peaks, i.c. the more reflective are the faces $x_{1} x_{1}^{\prime}$ and $x_{2} x_{2}^{\prime}$, the more sensitive the process. Besides the process just described, the process known as the equivalent-thickness fringe may, likewise, be used. Let us assume that the faces $x_{1} x_{1}^{\prime}$ and $x_{2} x_{2}^{\prime}$ are no longer parallel. The optical thickness of the interferometer varies along the whole length of the plates and intensity changes in accordance with the law shown in Fig. 3.34. Instead of a uniform intensity, varying as $e$ is altered while the alignment of $x_{1} \cdot x_{1}^{\prime}$ and $x_{2} \cdot x_{2}^{\prime}$ is retained, it is the intensity on the plates that changes. The interferometer then depicts the phenomenon shown in the curve (Fig. 3.34). The very dark field is streaked by very narrow shiny fringes correlating the peaks shown in Fig. 3.34. These fringes are straight and parallel to the edge of the prism formed by the faces $x_{1} x_{1}^{\prime}$ and $x_{2} x_{2}^{\prime}$. This no longer obtains in the area where the object lies as the optical thickness has changed and the fringes are now distorted (Fig. 3.35). This distortion, shown as inter-fringes, evinces 
the object's optical thickness. Figure 3.34 shows that, changing over from one bright fringe to the other, the optical thickness varies by $\lambda$. In the case depicted in Fig. 3.35, the object halves the fringe distance: therefore, the difference between its optical thickness and the medium encompassing it is $\lambda / 2$.

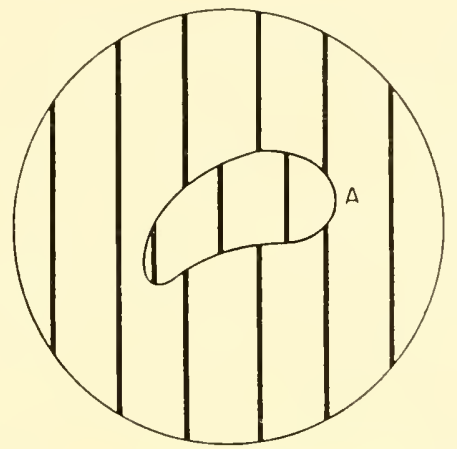

FIG. 3.35. Measurements with interference fringes.

Since the object is to be placed in the interferometer, the latter must be ahead of the microscope objective. The two plates $L_{1}$ and $L_{2}$ may consist of the cover-slip and the object-slide, respectively. The object to be examined is set between both plates, with their semireflective faces opposite. The optical quality of both cover slip and object-slide is very poor but the microscope fields are small enough as to consider the investigated area as bounded by plane and paralle

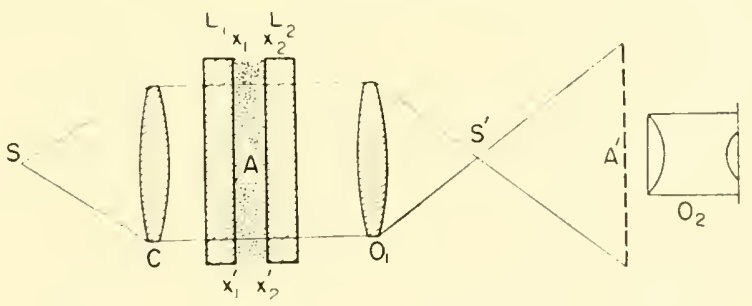

FIG. 3.36. Multi-wave interference microscope.

faces. The interference microscope, based on the foregoing, is shown in Fig. 3.36. The small source $S$ is placed in the focal plane of the condenser (in fact, it is the minute aperture of the condenser diaphragm upon which the source is imaged). The merging light forms a beam of parallel rays that pass through the interferometer $L_{1} L_{2}$ and the objective $O_{1}$, which images the transparent object $A$ at $A^{\prime}$. 
The drawbacks of interference microscopes of this type are the $f$ llowing: a very minute space between $L_{1}$ and $L_{2}$ (cover-slip and object-slide) is not feasible. Now, the interference theory shows that the thicker the interferometer, the stricter the source's monochromatic requirements must be. For instance, the microscope, shown in Fig. 3.36, is illuminated by a mercury-vapour lamp provided with a filter isolating the green spectrum-line. The source $S$ is to be of small size, as using a large source would cause many-angled rays to pass through $L_{1}$ and $L_{2}$. Now, for a given tilt the interferometer has a given optical thickness and the intensity of the phenomenon is, likewise, well determined. At another angle of tilt, the intensity varies as a function of the interferometer optical thickness. A whole series of diverse contrast phenomena are thus superimposed. The image contrast is poor and the sensitiveness of the method decreases rapidly.

Sir Thomas Merton suggested a method permitting the use of a large source. Let us consider again Fig. 3.36, where the surfaces $x_{1} x_{1}^{\prime}$ and $x_{2} x_{2}^{\prime}$ are assumed to be quite parallel. It is well known that. in monochromatic light, a plate of selfsame thickness exhibits fringes endlessly (or identically-angled fringes) which are ring-shaped.

They can actually be observed in the focal plane $S^{\prime}$ of the microscope objective $O_{1}$. They are also perceived at $S$, as virtual images, however. Let us assume that a diaphragm, pierced with annular apertures is set at $S$, these apertures being of such size and in such position that each one isolates a homologous portion in every lightfringe. Under such conditions, all the rings in the plane $S$ illuminate the specimen and give rise to identical phenomena. To every ring is related an image whose contrast is definite; all rings impart the same contrast to the image. Employing a diaphragm pierced with suitable rings, placed at $S$ affords an image whose contrast is as good as that of a pin-point source but with a greatly enhanced brightness and improved quality.

There is yet another stumbling block in connection with multiwave interference microscopes: with high N.A. objectives, the slant of the rays passing through the specimen becomes considerable and multiple reflections for these rays are no longer present: a siceplyslanting ray passing through the transparent object is more and more removed from the incident ray as reflections occur. If the object is not very thin, after one or two reflections, the ray issues from the transparent object; multi-wave interferences do not take place any longer and sensitiveness drops. 
Multi-wave interference microscopes do not give a very good performance when small objects are involved. In actual practice, it seems that $0.004 \mathrm{~mm}$ is the lowest width permissible for accurate scrutiny. However, for low- and medium-power magnification these instruments give excellent results.

\section{MENZEL'S INTERFERENCE MICROSCOPE}

The Menzel method is an extension to microscopy of the 3-slit method devised by Vaïsala, resumed later by Zernike.

Three small and narrow slits are imaged in the specimen plane. Let us assume that measurement of the path difference between the areas $A$ and $B$ is required (Fig. 3.37). The sub-stage is moved so that the 3 slits imaged are arranged as shown in Fig. 3.37. Two of these

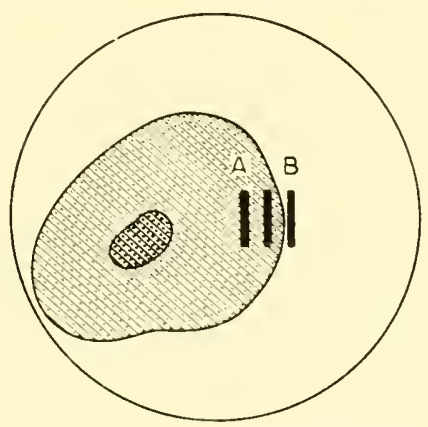

FIG. 3.37. Menzel's method.

images may lie in the area $A$ and one in the area $B$. Instead of noting measurements through observation of the specimen itself, it is the phenomena, taking place close to the focal plane of the microscope objective, which are scrutinized. To do this, a Bertrand lens is inserted between objective and eyepiece. The associated lens and eyepiece form a small sight providing focusing in the area of the objective's focal plane.

If there is no object (empty specimen) the interference fringes exhibit a typical appearance. Their position is close to the objective's focal plane. When the specimen is placed as shown in Fig. 3.37, the vibrations diffracted by the slit located at $B$ lead or lag in relation to the vibrations diffracted by the two slits at $A$. The upshot is a shift of the typical fringes along the microscope centre-line. Such motion is measurable through the focusing telescope (eyepiece plus Bertrand lens) by noting the altered position of these fringes. This will be 
achieved by merely shifting the focusing telescope only not before the typical fringes are identified anew. The motion of the fringes is ascertained by noting the focusing telescope motion and from these data the path difference between the areas $A$ and $B$ can be educed.

\section{SENSITIVITY OF INTERFERENCE MICROSCOPES}

An interference microscope provides the visible image of a phase object whose contrast depends on the path difference brought about by said object. The image contrast decreases as does the path difference. Below a given value, the image vanishes. The smallest path difference that may thus be shown also evinces the instrument's sensitiveness.

Accurate numerical values can hardly be given as sensitivity may be affected by factors quite foreign to the procedure used, e.g. stray light, aberrations, depth of focus and so forth. Under the best concitions, a sensitivity of approximately $\lambda / 1000$ is achievable with twowave interference methods. This means that, in transmitted light, a transparent object of optical thickness ne, immersed in a medium of index $n^{\prime}$ can be detected when $\left(n-n^{\prime}\right) e=\lambda / 1000$. This performance datum, however, does not apply to differential-method microscopes (\$ 7 and 8) as, then, sensitivity depends on the image duplication selected. Under very good conditions, the slope $\theta$ of a transparent object of index $n$, immersed in a medium of index $n^{\prime}$

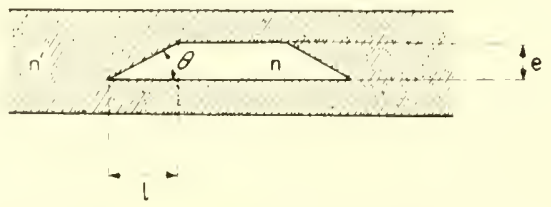

FIG. 3.38. Slop measurement (0) of a transparent object.

(Fig. 3.38) can be detected if $0\left(n-n^{\prime}\right)=\lambda / 1000 d$, where $d$ is the image-duplication brought about by the polarizing interferometer. An object of thickness $c$, whose slope 0 is of width $l$, is detectable, provided $\left(n-n^{\prime}\right) e=\lambda / 1000 \mathrm{~d}$. Sensitivity increases if the object width $l$ decreases but $d$ must remain small in relation to $l$.

Theoretically, multi-wave interference methods are not more sensitive than two-wave ones. However, the former provide the same measuring accuracy much more readily than do the two-wave instruments. We have already seen, in the field of microscopy, the drawbacks and magnification limitations of multi-waves devices. 


\section{CHAPTER IV}

\section{Reflected-light Microscopy}

\section{METHODS APPLIED IN REFLECTED-LIGHT MICROSCOPY}

THE illumination arrangement in reffected-light microscopes differs from that of transmitted-light microscopes. Figures 4.1 and 4.2 show the two principal arrangements.

In Fig. 4.1 the light originated by the source $S$ is reflected by the $45^{\circ}$ slanted semi-reffecting plate $G$ (Beck illuminator), then passes through

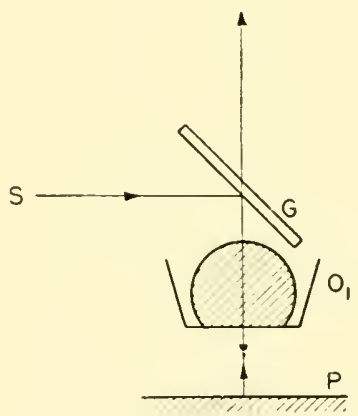

FIG. 4.1. Illumination with a semi-reflecting plate $G$.

the objective $O_{1}$, is reflected back by the object $P$ to the objective and passes through the plate in direction of the eyepiece.

The whole aperture of the objective is used; yet, the luminous flux reaching the eyepiece cannot exceed the theoretical 25 per cent maximum of the incident flux.

Figure 4.2 shows another illuminating arrangement comprising the mirror $M$, which occludes half of the objective's available aperture. One half of the objective serves as condenser, the other half as objective. This entails, hence, reduced definition. Furthermore. this arrangement exhibits an oblique illumination which may bring about 
illumination changes tending to obfuscate interpretation if the object is concurrently a phase object of varying thickness (reflecting evenly the light) and an amplitude object (featuring illumination or colour changes).

Figure 4.3 shows that the Köhler illumination is applicable to both methods. The lens $L_{1}$ images the source $S$ on the lens $C_{1}$. The lens $C_{2}$ then images $C_{1}$ at $F^{\prime}$, which is the focus of the objective $O_{1}$,

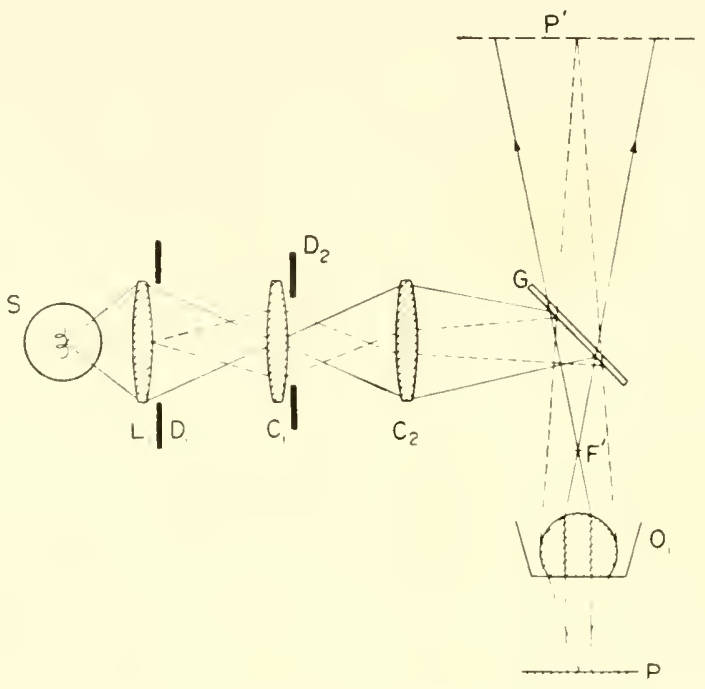

FIG. 4.3. Kohler illumination in reflected light.

after being reflected in $G$. The object $P$ is thus evenly illuminated. The lenses $C_{1}, C_{2}$ and the objective $O_{1}$ image the field diaphragm $D_{1}$, placed against $L_{1}$ on the object $P$. The whole system $S L_{1} C_{1} C_{2} G$ forms an element integral with the microscope and is a vertical illuminator. In other layouts, the vertical illuminator consists of $C_{1} C_{2}$ and $G$ only. The source $S$ and the lens $L_{1}$, belong to an independent system.

The diaphragm $D_{2}$ is placed against $C_{1}$ (diaphragm aperture) in order to vary the illumination angle. The system that exhibits the most stray light is that shown in Fig. 4.1. A ray such as $S I J$ (Fig. 4.4) is reflected at $J$ in one of the objective's lenses and reverts to the eyepiece. The stray light is originated owing to glass reflection from the surfaces of the objective's lenses which may attain 7 in number in powerful achromats. Stray light is, therefore, substantial: much more so than in transmitted-light microscopes as, in the latter, the 
stray rays are originated from at least 2 successive glass reflections which weaken them markedly. In metallographic microscopes, stray rays are originated from a single reflection and, hence, more intense.

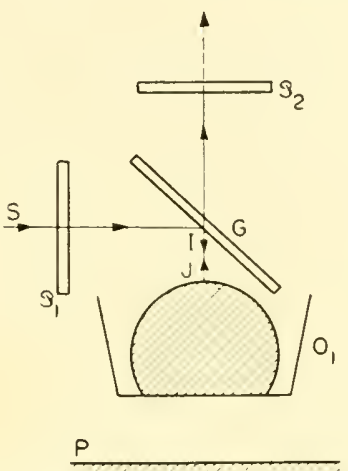

FIG. 4.4. Stray light reflected by the lenses of the objective.

The device, shown in Fig. 4.2, does away with a substantial part of the stray light, which is intercepted by the mirror $M$. However, its drawbacks were evinced elsewhere.

The greater part of the stray light is virtually originated from the reflection in the central part of the objective's lenses. Leitz observed this and developed a device reducing stray light materially. When using this device, illumination is originated solely from the objective's periphery. This annular illumination increases the microscope's sensitivity in observation of micro-relief. Another device, perfected by Zeiss, makes use of polarized light. The polarizer $\mathscr{L}_{1}$ and the analyser $\mathscr{L}_{2}$ are placed ahead of $G$ and of the eyepiece, respectively. The polarizer $\mathscr{L}_{1}$ is so arranged that the vibration it transmits is either at right angles to or in the incidence plane $S I J$ related to $G$. Under such conditions, the reflection on $G$ does not alter anything and the vibration remains straight after impinging on $G$. If the analysers $\mathscr{L}_{2}$ and $\mathscr{L}_{1}$ are crossed, the light reflected by the lenses of the objective $O_{1}$ is fully occluded. Therefore, there is no stray light any longer. But the light, reflected by the object $P$, is occluded, too, and must re-appear. In the method propounded by Zeiss (Fig. 4.5), the quarter-wave plate $Q$ is placed against the objective and, being gone through twice, becomes half-wave. The vibration, reflected by $P$, rotates $90^{\circ}$ and may then pass through $\mathscr{L}_{2}$. Nomarski suggested using, instead of a half-wave plate, a parallelto-the-axis quartz plate. whose thickness is adequate to show a chan- 
nelled spectrum between polarizers. Five or six channels are adequate. The device then lets through some higher-order white light. The plate is half-wave but not for a single wave-length only: it applies to a whole series of wave-lengths distributed within the spectrum. The device is

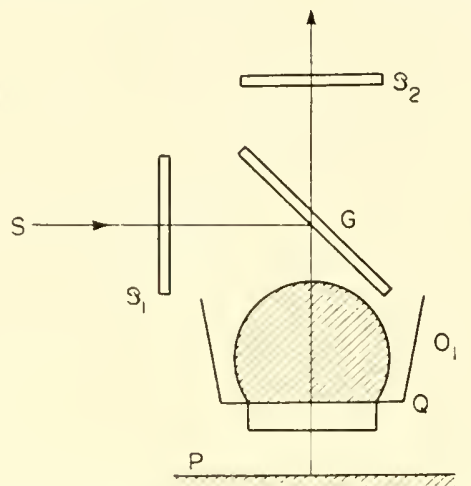

FIG. 4.5. The light reffected by the object $P$ re-appears with the aid of a quarter-wave plate $Q$.

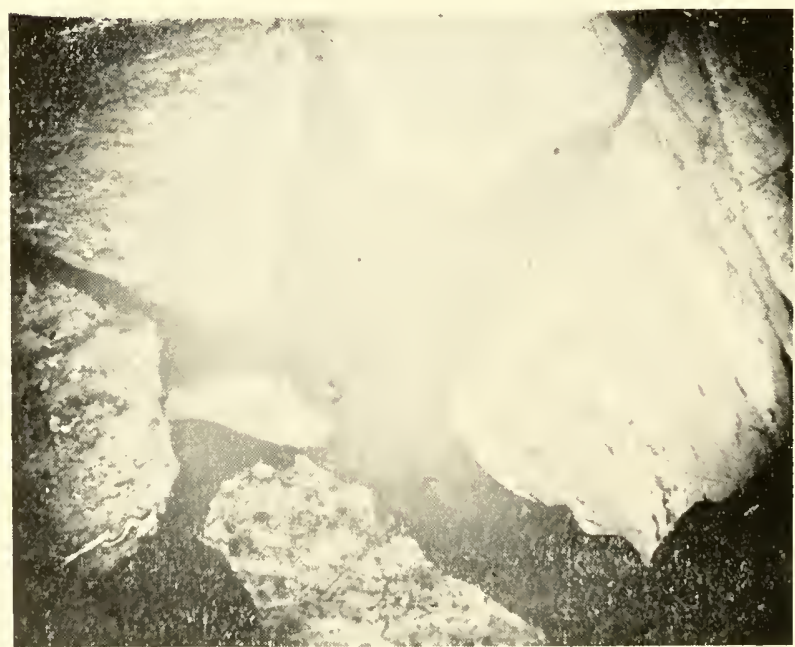

FIG. 4.6. Carbon observed with a standard objective (G. Nomarski).

truly achromatic. Figures 4.5 and 4.6 show the same object, observed with an illuminator of the type shown in Fig. 4.1, with and without quartz plate (Fig. 4.7).

Considerable advances have been made, not only from the straylight standpoint, important problem in reflected-light microscopy, but 
also in observation methods and devices. Phase-contrast and interference methods have given rise to valuable potentialities. Long working distance objectives, mirror objectives or Dyson's device have

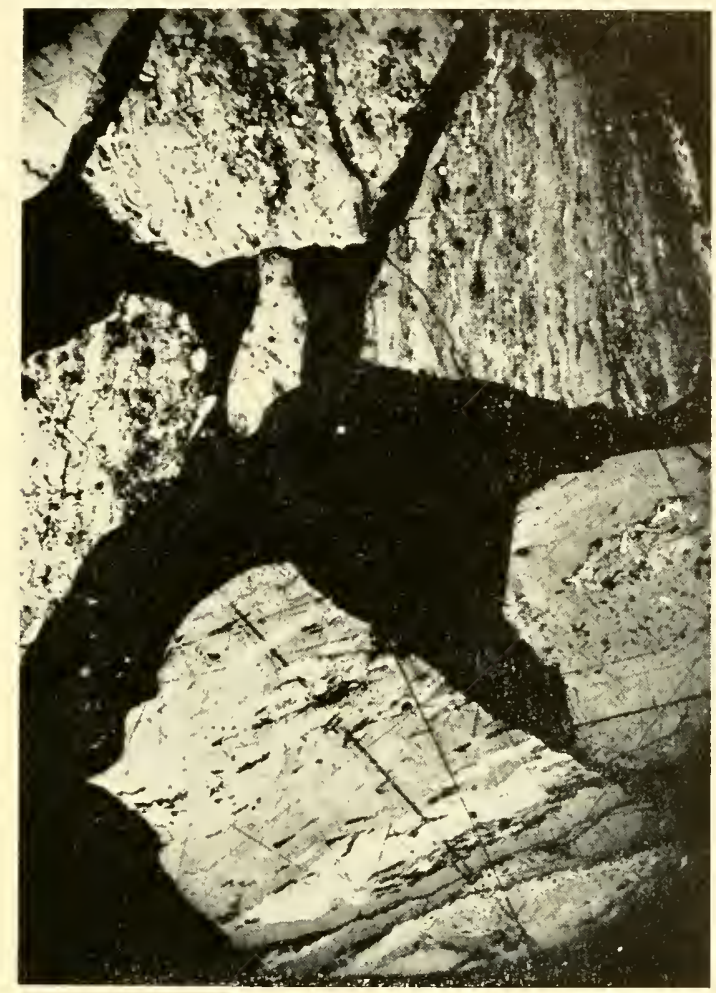

FIG. 4.7. Carbon observed with the Nomarski device.

provided the means to investigate high-temperature surfaces at comparatively substantial magnifying powers.

\section{REFLECTED-LIGHT PHASE-CONTRAST}

In general, surface flatness imperfections of metallographic sections exceed $100 \AA$. Such defects originated either from a difference in the conditions under which the mechanical removal of stock from the naterial took place when ground or to dissolution, occurring to a lesser or greater degree, during the electrolytic polishing process. The so-originated roughness is plainly visible in phase-contrast microscopy. For instance, the pearlitic structure of grey irons shows, in phase-contrast microscopy, highly-contrasted images enabling one to 
determine the grain size and to devise differences that may be correlated with constituents of various hardness numbers.

F. W. Cuckow showed that phase-contrast and electron microscopy images are closely alike: in a chrome-nickel steel the phase-contrast dark areas are correlated with the dark areas of an impression observed in the electron microscope and hence, to hollows in the cross-section. Cross-sectional differences in levels of flat metallographical sections are further magnified by etching. F. W. Cuckow and D. McLean showed that moderate etching affords much more detailed images in phase-contrast microscopy.

Therefore, phase-contrast microscopy is a valuable tool in metallography and, in general, when investigating non-diffusing reflecting objects.

The Jupnik, Osterberg and Pride phase microscope (American Optical Company)

The object $P$ is illuminated (Fig. 4.8) by a vertical illuminator set next to the objective $O_{1}$, as in ordinary reflected-light microscopes.

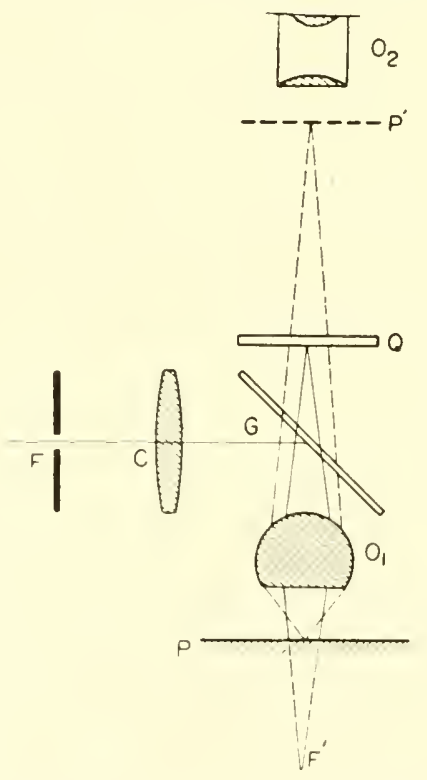

FIG. 4.8. Jupnik, Osterberg and Pride device.

The source $F$, which is, in fact, a diaphragm upon which is imaged a lamp filament, may be shaped either as a ring or a slit.

In order not to over-elaborate Fig. 4.7, a slit in shown. The light, 
originated from $F$, passes through the lens $C$, is reflected on the $45^{\circ}$ slanted plate, passes through the objective $O_{1}$ and is reflected on the object $P$. Thus is the diaphragm $F$ imaged under the object at $\dot{F}^{\prime}$. The light-rays pass again through the objective $O_{1}$ which images $F^{\prime}$ on the phase-plate $Q$. The image $P^{\prime}$ of 'object $P$ is observed through the eyepiece $\mathrm{O}_{2}$.

The Françon and Nomarski layout

The variable-absorption reflecting phase-plate (Chap. II, § 6), can be used for reflected-light observation. Figure 4.9 shows the instrument's layout. The illuminator, similar to the one shown in

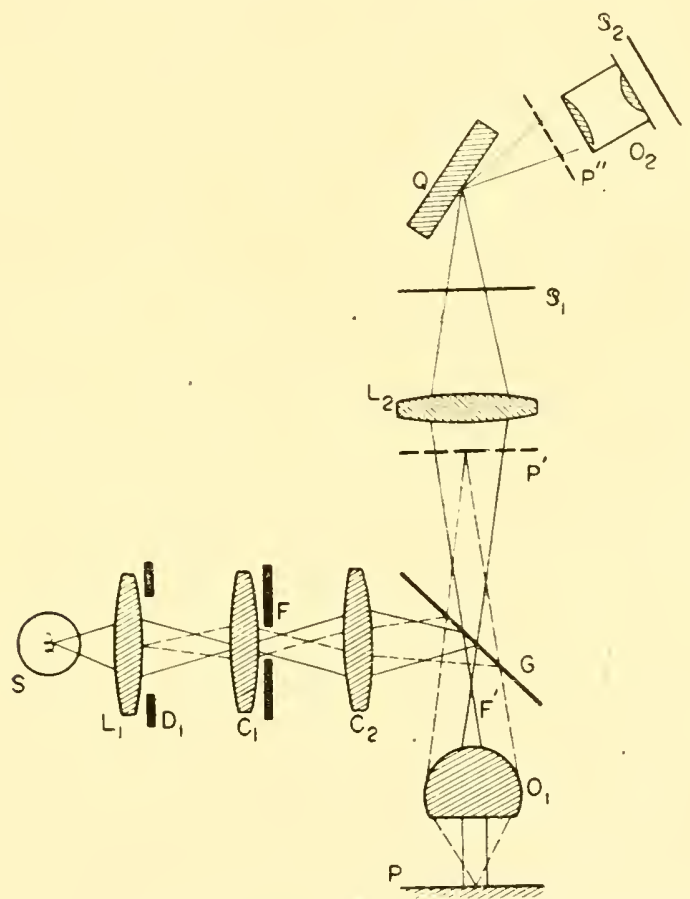

FIG. 4.9. Françon-Nomarski device.

Fig. 4.3, comprises the slit $F$ next to the lens $C_{1}$. The lens $C_{2}$ images the slit $F$ at $F^{\prime}$. This image is in the focus of the objective $O_{1}$ and the rays, illuminating the specimen $P$, form a beam of parallel rays. The rays are reflected back by $P$ on the lens $L_{2}$ which images $F^{\prime}$, i.e. $F$, on the phase-plate $Q$. The lens $L_{2}$, is so arranged as to image $P$ at $P^{\prime \prime}$ after being reflected on the phase-plate. 


\section{The Bendford and Seidenberg layout (Bausch and Lomb)}

The instrument is shown diagrammatically in Fig. 4.10. The source $S$ is projected on the annular diaphragm $D_{1}$ which is imaged at $F^{\prime}$, after being reflected in the prism $R_{1}$, passing through the lens $C$ and the $45^{\circ}$-slanted plate $G$. The focus of the objective $O_{1}$ coincides with $F^{\prime}$ so that. for every point of the source, the light-emerging

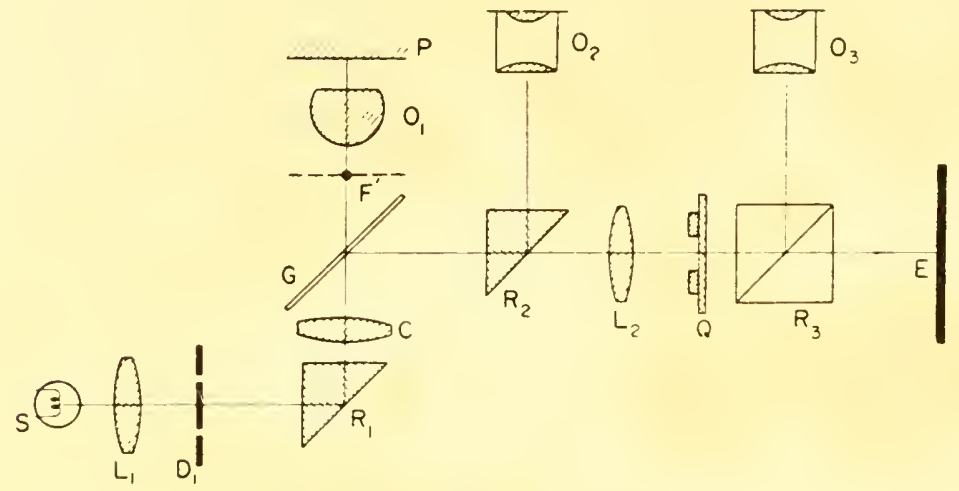

FIG. 4.10. Bendford and Seidenberg device.

from the objective $O_{1}$, forms a parallel beam. The rays are reflected by the reflective object $P$, pass through the objective $O_{1}$ and are reflected on $G$. The retractable prism $R_{2}$ reflects the light into the eyepiece $O_{2}$ designed to observe the image without phase-contrast. The prism $R_{2}$, being removed, the light passes through the optical system $L_{2}$ which images $F^{\prime}$, i.e. the diaphragm $D_{1}$, on the phase-plate $Q$. Next, the light passes into the cube $R_{3}$ which splits it in two equal paris: the first passes through the eyepiece $O_{3}$, the other proceeds to the photographic plate $E$. The image, observed in the eyepiece $O_{3}$. and the photographic-plate image, are both phase-contrasted. The device is so adjusted that the object $P$, imaged by the objective $O_{1}$. is focused simulaneously on the photographic plate and in the eyepiece $\mathrm{O}_{3}$.

\section{OBLIQUE ILLUMINATION AND DARK GROUND IN REFLECTED LIGHT. SCHMALTZ'S SLIT}

In reflecting microscopes, oblique illumination enables one to detect surface unevennesses. In Schmaltz's method, a slit is imaged by the optical system $C$ (Fig. 4.11) on the surface to be studied $P$. The axis 
of the observation microscope objective $O_{1}$ is at right angles to the optical system $C$. The observed surface $P$ is, therefore, illuminated at a $45^{\circ}$ angle. Let us assume that, in some area of the field of view, the sample surface is at $P$. In this area, the slit is imaged at $A$. If, in

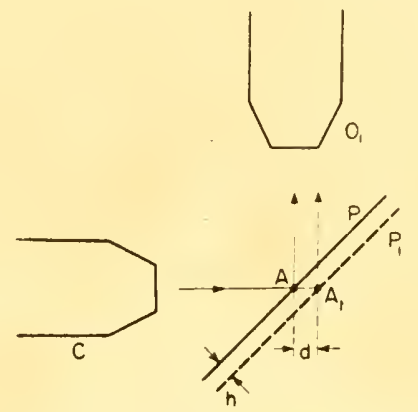

FIG. 4.11. Schmaltz's method.

another area, the surface is at $P_{1}$, the image slit is visible at $A_{1}$. The difference in level $h$ is shown by the lateral motion $d=h, 2$ of the imaged slit. The unevenness shown in Fig. 4.12 is the strip-shaped extra thickness $A_{1}$, the remainder of the object $(A)$ being flat.

Owing to the instrument's layout, the space between the objectives and the object $P$ is always large. This fact makes the use of

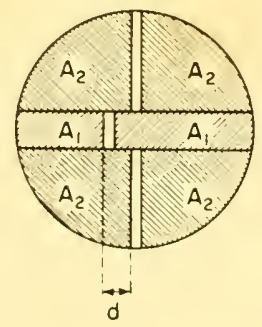

FIG. 4.12. Image aspect in Schmaltz's method.

objectives, whose focal length is less than $16 \mathrm{~mm}$, hardly feasible. Only low magnifying powers are usable. Schmaltz's layout was inproved by Busch and Tolansky who designed the microscope objective $O_{1}$ so as to illuminate and be used for observation at the same time (Fig. 4.13). The source $S$ is imaged by the lens $L$ in the slit $F$. The rays originated from $F$ are reflected by the mirror $M$, pass through the objective $O_{1}$ and after being reflected in the object $P$, form the 
image $P^{\prime}$ originated from the objective $O_{1}$. Owing to the mirror $M$, the rays impinging on $P$ form an oblique beam. The space from the slit $F$ to the objective $O_{1}$ is to be same as that from the image $P^{\prime}$ to

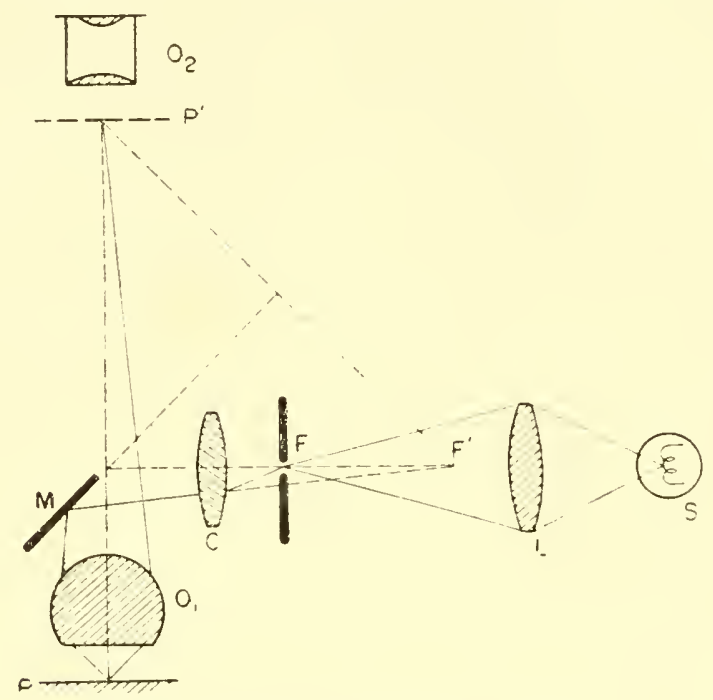

FIG. 4.13. Improved Schmaltz's method.

the objective $O_{1}$. To save space, a lens is provided at $C$, which forms a virtual image of $F$ at $F^{\prime}$ at the right distance. Figure 4.14 shows

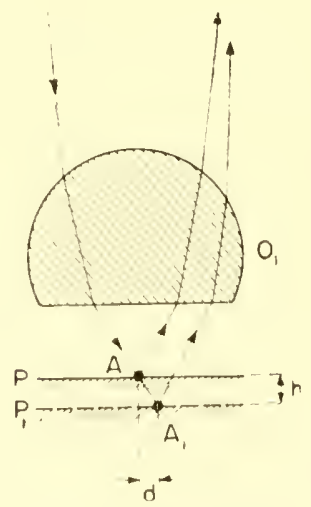

Fig. 4.14. Improved Schmaltz's method.

that the difference $h$, between the levels $P$ and $P^{\prime}$, is given by the sidewise shift $d=h \tan \alpha$ where $\alpha$ is the incidence angle on the investigated surface. 


\section{Menzel's method}

Menzel substitutes Schmaltz's slit for a special transparent plate (Fig. 4.15) which consists of a series of plates each of which gives rise to a $\pi / 2$ phase-shift (half-wave path difference) in relation to the

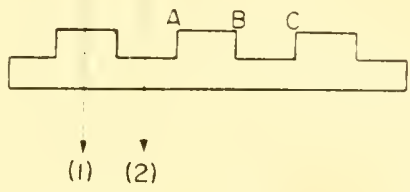

FIG. 4.15. Menzel's method.

next ones. A ray, such as (1), lags one-half wave-length in relation to a ray such as (2). The plate is set in the vertical illuminator so that it is not imaged on the object but some distance away from it.

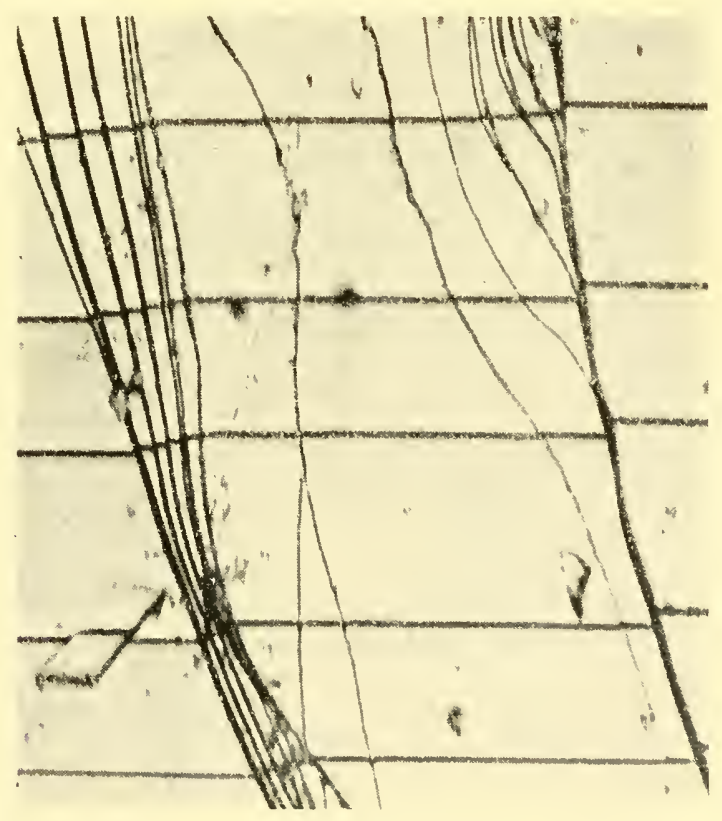

FIG. 4.16. Observation of cleavage planes of mica (courtesy of Prof. Menzel).

The microscope layout is the same as the one shown in Fig. 4.13. Therefore, when focusing the object the Menzel plate is out of focus. Investigation of finite-distance diffraction phenomena (Fresnel's phe- 
nomena) shows that a small break between two areas, whose path difference equates $i / 2$, exhibits, through faulty focusing, a perfectly black diffraction fringe. Such a fringe is exhibiled, in Menzel's plate, by discontinuities. such as $A, B$, $C$, etc. A series of fine dark fringes (highly convenient for observation), are thus perceived on the object. Figure 4.16 shows a photograph obtained by this ingenious layout.

\section{Dark ground}

Surface unevennesses may also be derected by the dark ground.

Figures $4.17,4.18,4.19$ show the layout of several devices. Lieberkunn's arrangement is only suited to low magnifying powers. The

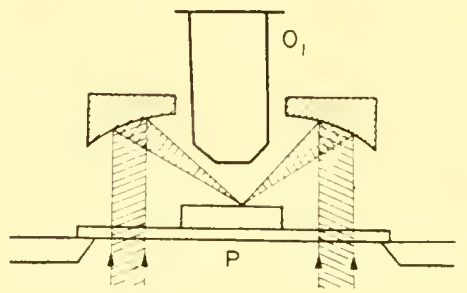

FiG. 4.17. Licberkuhn device.

Chapman and Alldridge device (Fig. 4.18) and Reichert's (Fig. 4.19) are catoptric devices. Leitz’s ultropak (Fig. 4.20) employs a dioptric

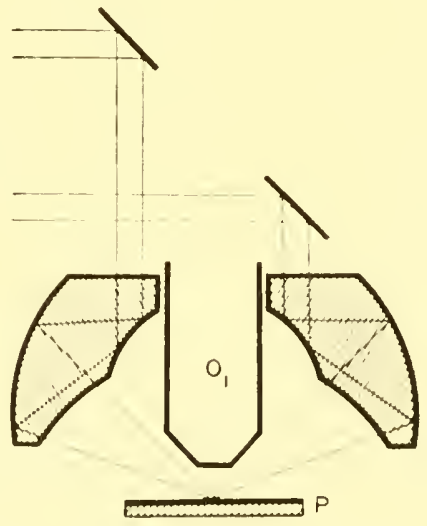

FIG. 4.18. Chapman and Alldridge device.

layout. In these instruments the incident light does not pass through the objective. If the object $P$ is perfectly flat, the incident rays are not deflected from the paths shown in Figs. 4.17, 4.18. 4.19 and 4.20. 
No light passes into the objective. A surface unevenness in the object diffracts light which enters the objective. The unevenness is bright on a dark ground. Measuring the surface slopes is facilitated by using

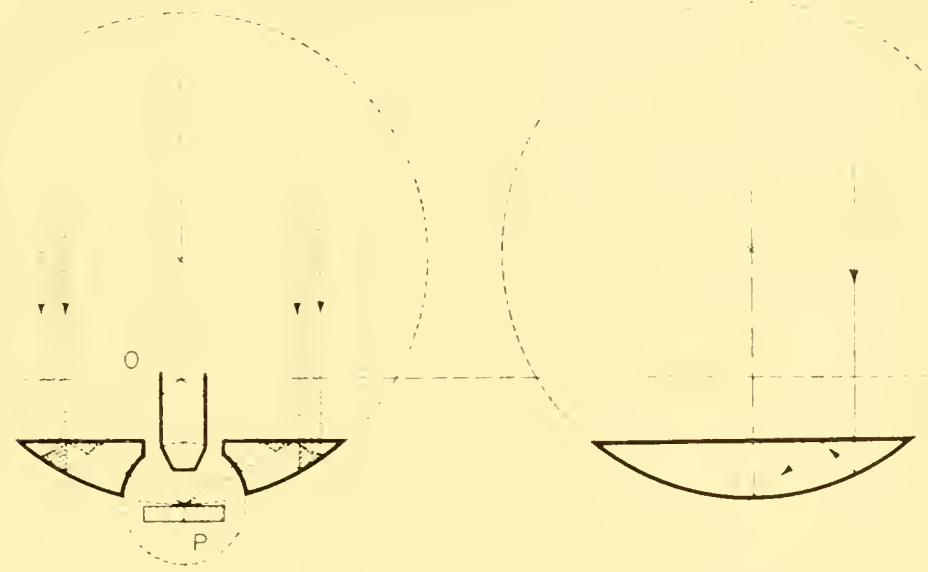

FiG. 4.19. Reichert's device (Epilum).

a pin-point source whose image is occluded by an opaque screen (Fig. 4.21). The pin-point source is imaged at $F^{\prime}$ in the focus of the

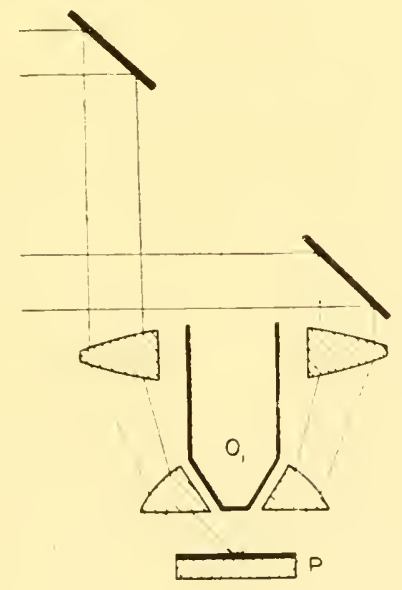

FIG. 4.20. Leitz's ultropak.

microscope objective $O_{1}$. The rays, emerging therefrom, form a beam of parallel rays which, after being reflected on the surface $P$, image the source $F^{\prime}$ at $F_{1}^{\prime}$. In Fig. 4.21 , the opaque screen $D$ is shifted to 
the left. While the screen $D$ is shifted, the image of $P$, given by $O_{1}$. is observed through the eyepiece (not shown in Fig. 4.21). As $D$ occludes the image $F_{1}^{\prime}$, the object $P$ is dark. Let us assume that, in another area of the field, the object exhibits the unevenness $P_{1}$, lying

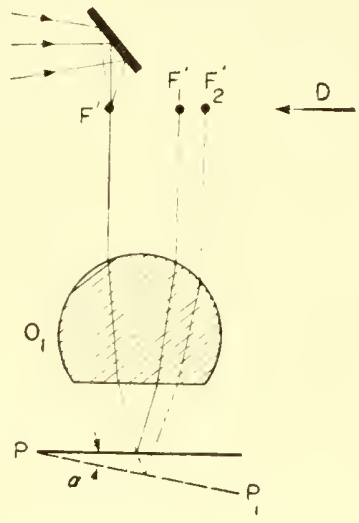

FIG. 4.21. Measuring the surface slopes.

at the angle $\alpha$ in relation to $P$. The image reflected by the area $P_{1}$ reaches $F_{2}^{\prime}$. In order to see the illuminated area the screen $D$ is to be shifted by the distance $d=F_{1}^{\prime} F_{2}^{\prime}$. If $f$ is the objective's focal length. the slope $\alpha$ is measured by the ratio $d / 2 f$.

\section{REFLECTED-LIGHT INTERFERENCE MICROSCOPES}

The interference methods described in Chapter III are applicable in reflected light.

Sagnac's interference microscope

G. Sagnac described one of the first interference microscopes in Le Radium and called it: "interference microstrioscope". The principle applied is that of Michelson's interferometer. The main element consists of a separator, formed by the two equal isoceles prisms $P_{1}$ and $P_{2}$ (Fig. 4.22). The two prisms are so arranged as to provide between them the parallel-sided, thin air gap $I^{\prime}$ and their angles are so selected that the intensitics of the beanis reflected and transmitted by the gap $I I^{\prime}$ are equated. An incident ray, such as $S I$, originates 
two rays, one reflected, $I R$, one transmitted, $I T$. The ray $I R$ is reflected by the object $A$, passes through the separator and enters the microscope's objective $O_{1}$. The transmitted ray $I T$ is reflected on the silvered side $M M^{\prime}$ of the prism $P_{2}$, then on the gap $/ l^{\prime}$ and enters the objective $O_{1}$. The wave, deformed by the unevennesses of the object $A$.

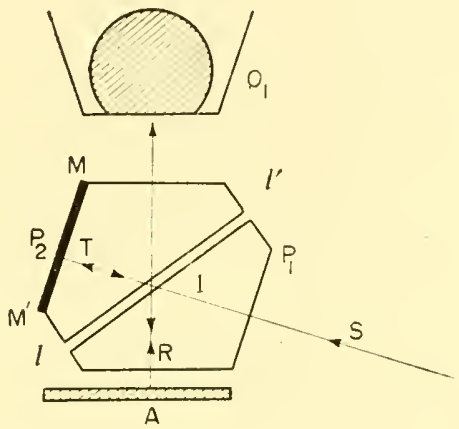

FIG. 4.22. Sagnac interference microscope.

interferes with the reference plane-wave reflected by $M M^{\prime}$. The unevennesses of the object $A$ are thus evinced. Linnik's, Krug's and Lau's microscopes are also based on the principle of the Michelson interferometer.

Linnik's interference microscope (Fig. 4.23.)

The incident light-beam from the vertical illuminator, schematized in Fig. 4.23, is split in two by the $45^{\circ}$, slanted separator $G$. Some of the light is reflected in the lower part of the figure, passes through the objective $O_{1}$ and illuminates the object $P$. It is then reflected back, passes again through $O_{1}$ and the separator $G$, and travels towards the eyepiece $O_{2}$. The light, transmitted by $G$, passes through the objective $O_{1}^{\prime}$ (which is identical with $O_{1}$ ), is reflected back by the mirror $M$, passes through $O_{1}^{\prime}$ again and is reflected back by $G$ to the eyepiece $O_{2}$. Both images of the source $S, F_{1}$ and $F_{1}^{\prime}$, are formed in the focal plane of the two objectives $O_{1}$ and $O_{1}^{\prime}$.

The wave $\Sigma_{1}$, deformed by the surface unevennesses of the object $P$, and the reference plane-wave $\Sigma_{1}^{\prime}$, interfere in the image $P^{\prime}$, seen through the eyepiece $O_{2}$. If the image of the mirror $M$, through reflection on $G$, is parallel with $P$, the field is uniform. Thickness unevennesses show up as light-intensity changes. Tilting $M$ slightly causes the 
image of $M$ to be no longer parallel with $P$ and straight fringes are developed. The greater the space between these fringes the wider the

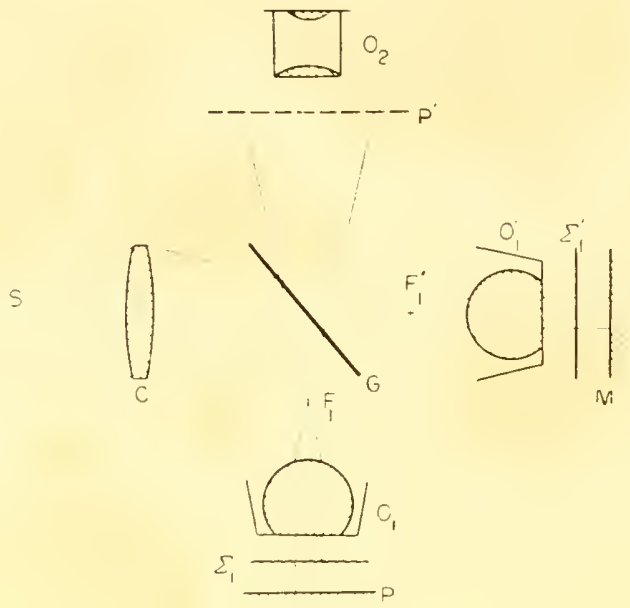

FIG. 4.23. Linnik interference microscope.

angle formed by the image of $M$ and $P$. Now, thickness unevennesses can be measured by shifting the fringes. This microscope is built in the U.S.S.R.

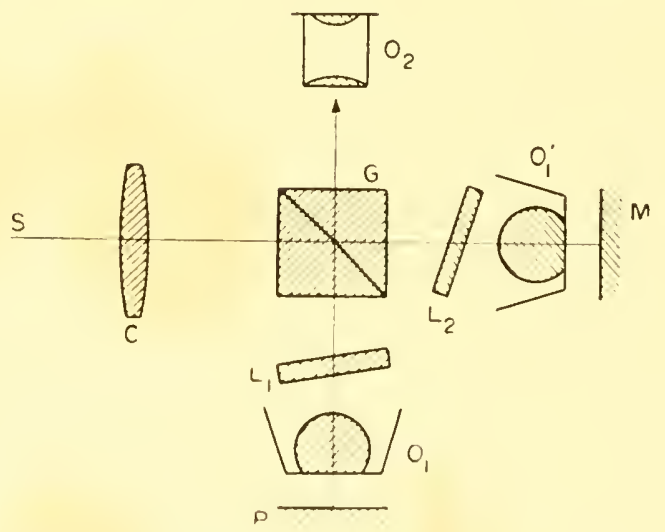

FIG. 4.24. Zeiss interference microscope.

Zeiss (K. Raentsch) has also designed a microscope based on this principle (Fig. 4.24). In this instrument. the direction and spacing of the fringes are adjusted by the two plates $L_{1}$ and $L_{2}$ set in the pupils of the 
objectives $O_{1}$ and $O_{1}^{\prime}$. Figure 4.25 shows a frosted and silvered glass surface, photographed by the Zeiss interference microscope.

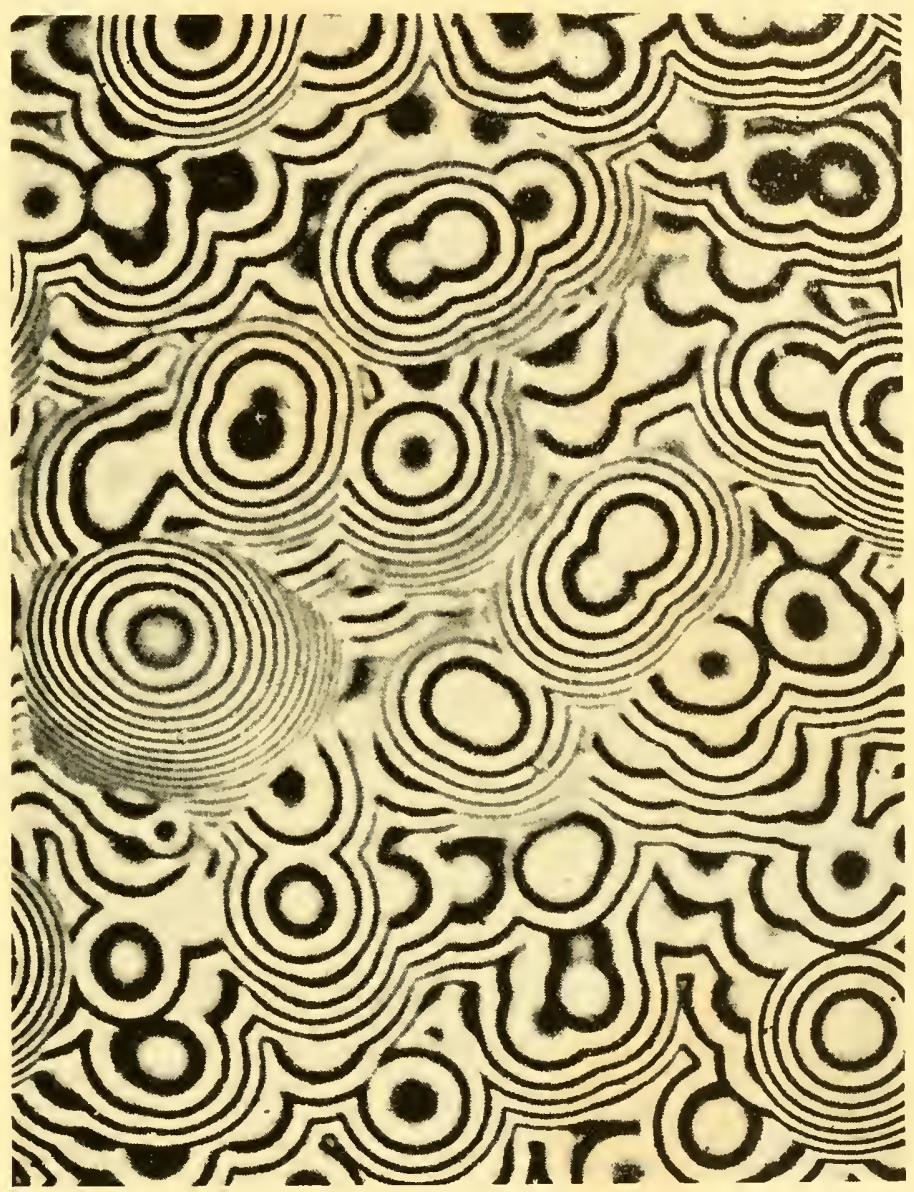

FIG. 4.25. Frosted and silvered glass surface photographed by the Zeiss interference microscope (Von Martin Uhlig. Courtesy of Zeiss).

The Krug and Lau interference microscope (Fig. 4.26) is also based on the same principle. The beams are split on the $45^{\circ}$-slanted semi-reflective face $G$, located in the prism $Q$. Rienitz also achieved particular interference microscopes based on a similar principle.

Hilger and Watt, and C. Baker, have also designed two-wave interference microscopes for investigating reflective objects. The Hilger 
and Watt microscope is based on Michelson's principle. Baker's microscope is based on either the Michelson or the Mirau principel.

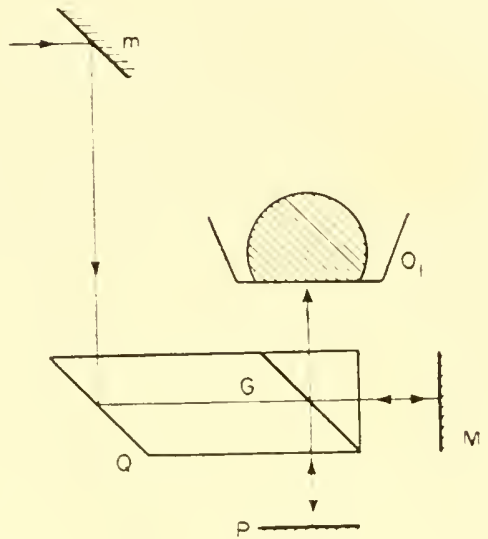

FIG. 4.26. Krug and Lau interference microscope.

Mirau's interference microscope (Fig. 4.27.)

The principle of this instrument is the following: the incident light-beam, emerging from the objective $O_{1}$, is split in two parts in the semi-reflective plate $G$. One part is reflected on the object $P$ and the other in the area $R$ of the front lens of the objective $O_{1}$. This

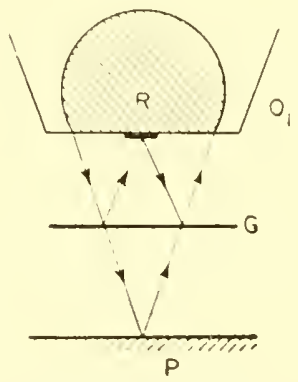

FIG. 4.27. Mirau interference microscope.

area. which extends over a small portion of the front surface, is made fully reflective by an opaque metal layer. Both beams are superimposed anew as they enter the objective $O_{1}$. The surface $R$ is the reference surface as was the mirror $M$ in Figs. 4.23 and 4.24. The horizontal plate $G$ is inserted half-wily between $R$ (front surface of 
the objective $O_{1}$ ) and the object $P$. Only low-aperture objectives are suitable in this application.

Dyson's interference microscope (Fig. 4.28.)

Three glass blocks cemented together, $B_{1}, B_{2}$ and $B_{3}$, are inserted between the objective $O_{1}$ and the object $P$. The block $B_{3}$ is a $15 \mathrm{~mm}$ radius-of-curvature plano-convex lens. The flat face $M M^{\prime}$ is semi-

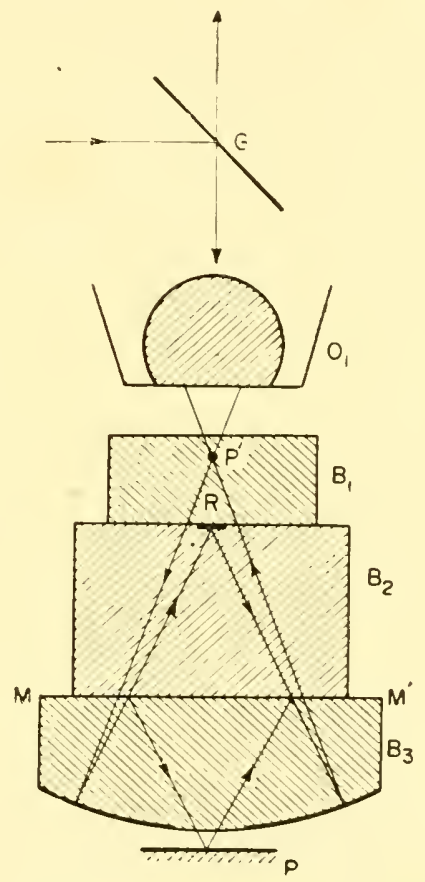

FIG: 4.28. Dyson interference microscope.

reflective. The convex side of $B_{3}$ is silvered, except in the polar area. The block $B_{2}$ is a parallel-sided plate on the top of which the small area $R$ is made highly reflective by an opaque metal layer. The utmost thickness of $B_{3}$ is slightly less than the radius of curvature of the convex surface. The thickness of $B_{2}$ is such that the curvature centre of the convex side is about $1 \mathrm{~mm}$ above $R$. The rays originated from $P$ are reflected back by the convex side of $B_{3}$ on to $P^{\prime}$ where $P$ is imaged by the system $B_{1}, B_{2}, B_{3}$. Then $P^{\prime}$ is observed through the objective $O_{1}$ of an ordinary microscope. The incident light illuminating $P$ travels in a direction opposite to the foregoing. After 
being reflected in the semi-reflective plate $G$ (the vertical illuminator is not shown) and, passing through the objective $O_{1}$, the ray enters the system $B_{1}, B_{2}, B_{3}$ and, next, is reflected by the convex side and splits in two as it impinges on $M M^{\prime}$. One of these rays is reflected on the object $P$ and the other on the mirror $R$. Both rays are superimposed anew as they impinge on the convex side. The reflected beam reverts to the objective $O_{1}$. As the image $P^{\prime}$ is not exactly in the centre of curvature of the convex side, some spherical aberration is developed which may be corrected by giving a suitable thickness to the block $B_{1}$. Thickness variations of approximately $30 \AA$ have been detected by the Dyson layout. Since the object is examined through reflection, optical paths are twice as long. Therefore, a $30 \AA$ optical path variation in the object correlates a $60 \AA$ variation as detected in the instrument. This remark applies equally well to any reflected-light instrument.

Françon's layout (Fig. 4.29.)

No mention need be made regarding the interference eyepiece described in Chapter III as merely setting this device in a reflecting microscope evinces interferential images of reflective objects. Fig-

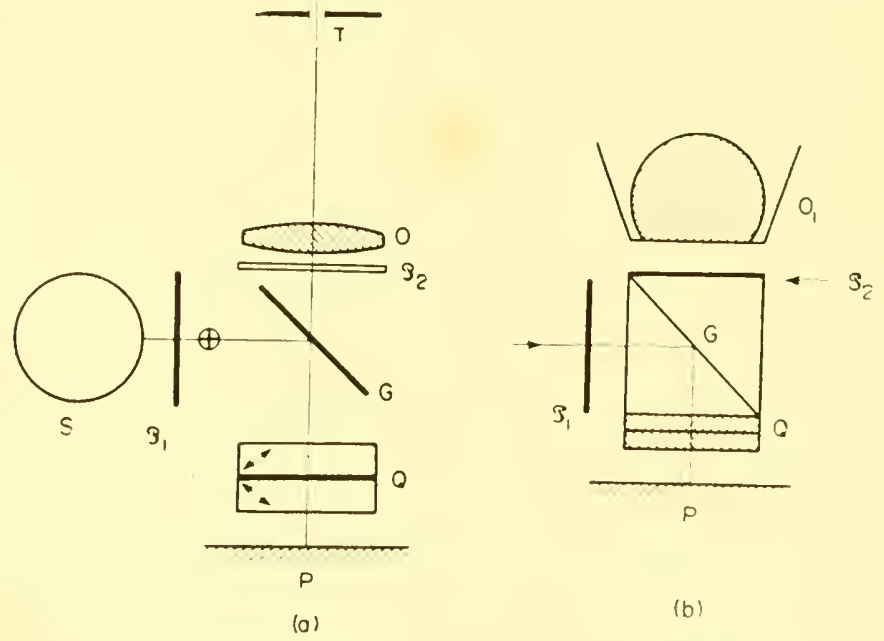

FIG. 4.29. Françon interference microscope.

ure 4.29 shows the principle of a different and simple polarizing interference device. Where low-magnifying power is involved, the observation device consists of a mere magnifying glass $O$ as shown 
in Fig. 4.29(a). The light is originated from the lamp $S$ (opal or diffusing); after being reflected on the $45^{\circ}$-slanted semi-reflective plate $G$ it passes through the polariscope $Q$ and is reflected by the object $P$ and reflected again on to the magnifying glass $O$ and the ocular diaphragm $T$ behind which is the observer's eye. This ocular diaphragm is in the focus of the magnifying-glass $O$. In order to focus, the object $P$ is shifted in relation to the instrument whose elements are all secured together. The polaroid element $\mathscr{L}_{1}$ is so arranged as to emit a vibration at right angles to the incidence plane on $G$. The second polaroid element $\mathscr{L}_{2}$ is crossed in relation to $\mathscr{L}_{1}$. Hence, were it not for the presence of $Q$ no light reaches $F$. The polariscope $Q$ is not of the Savart type. It consists of two quartz plates, cut $45^{\circ}$ from the optical axis and whose axes are in the same plane and at right angles. A Cellophane half-wave plate is cemented between the two plates, $45^{\circ}$ from the quartz axes. A single passage of a light-beam through this polariscope gives rise to on-infinity fringes which are parallel and equidistant straight lines (the on-infinity Savart fringes are only approximately straight lines). Two passages of the light beam cause the on-infinity fringes to overspread completely and the tint is flat. There is no need for any diaphragming and the lamp is accordingly used without a collimator.

According to the thickness of the polariscope $Q$ either the fullduplication or the differential method may be used (Chap. III, $\$ 5$ ). The same device is also applicable to a low magnifying power microscope (Fig. 4.29(b)). In this case, the system $G$, similar to that shown in Figs. 4.23, 4.24 and 4.25 is inserted between the objective $O_{1}$ and the polariscope $Q$. It should be noted that the layouts shown in Figs. 4.29(a) and (b) are completely devoid of stray light: any light-beam which has not passed through $Q$ is occluded since $\mathscr{L}_{1}$ and $\mathscr{L}_{2}$ are crossed. The light reflected by the underface of $Q$ is also occluded since, if $Q$ is set in the proper direction, the light passing through $Q$ twice and which is reflected against its underface exhibits on-infinity fringes that are approximately zero at $T$. Therefore, there is no reflected stray light in the eyepiece-lens plane. Only the light reflected back by the object $P$ reaches the observer, as shown in Chapter III.

Nomarski's device (Fig. 4.30.)

A Wollaston prism $W$, set in the objective's focus, is used in this instrument. The light originated from the vertical illuminator is 
polarized by the polaroid element $\mathscr{D}_{1}$. After being reflected on the mirror $M$ the source is imaged on $W$ at $I$. Since $I$ is in the focus of the objective $O_{1}$, the rays emerging from the latter constitute a beam of parallel light. The rays are reflected back from the object $P$ and the source is imaged anew in $W$ at $J$. The beam then passes through the polaroid element $\mathscr{L}_{2}$ and proceeds to the eyepiece $O_{2}$. Let us assume that $I$ and $J$ are symmetrical in relation to the centre of the

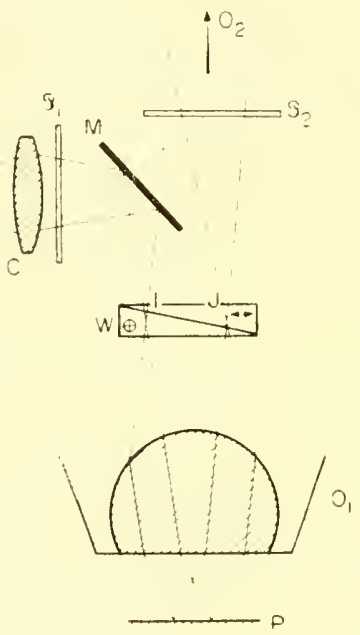

FIG. 4.30. Nomarski interference microscope.

Wollaston prism whose two elements have the same thickness. The path difference between the ordinary and the extraordinary ray at $I$ is the same as at $J$ but these differences are of opposite sign and cancel out. This property is retained irrespective of the extent of the source imaged at $I$ as, were the Wollaston centre to lie in the optical axis of the microscope and the plane of the object $P$ be perpendicular to said axis, a point $J$, symmetrical in relation to the Wollaston centre, is correlated with any random point $I$. Thus an extended source may be used without diaphragming the vertical illuminator. The path differences do not cancel out any longer if the object $P$ is slightly tilted or the Wollaston moved in its plane. Nothing is altered and the vertical illuminator may remain at full aperture. Figure 4.30 is a theoretical diagram. In actual practice, the Wollaston prism is substituted for a prism similar to the one shown in Fig. 3.26. The layout can there- 


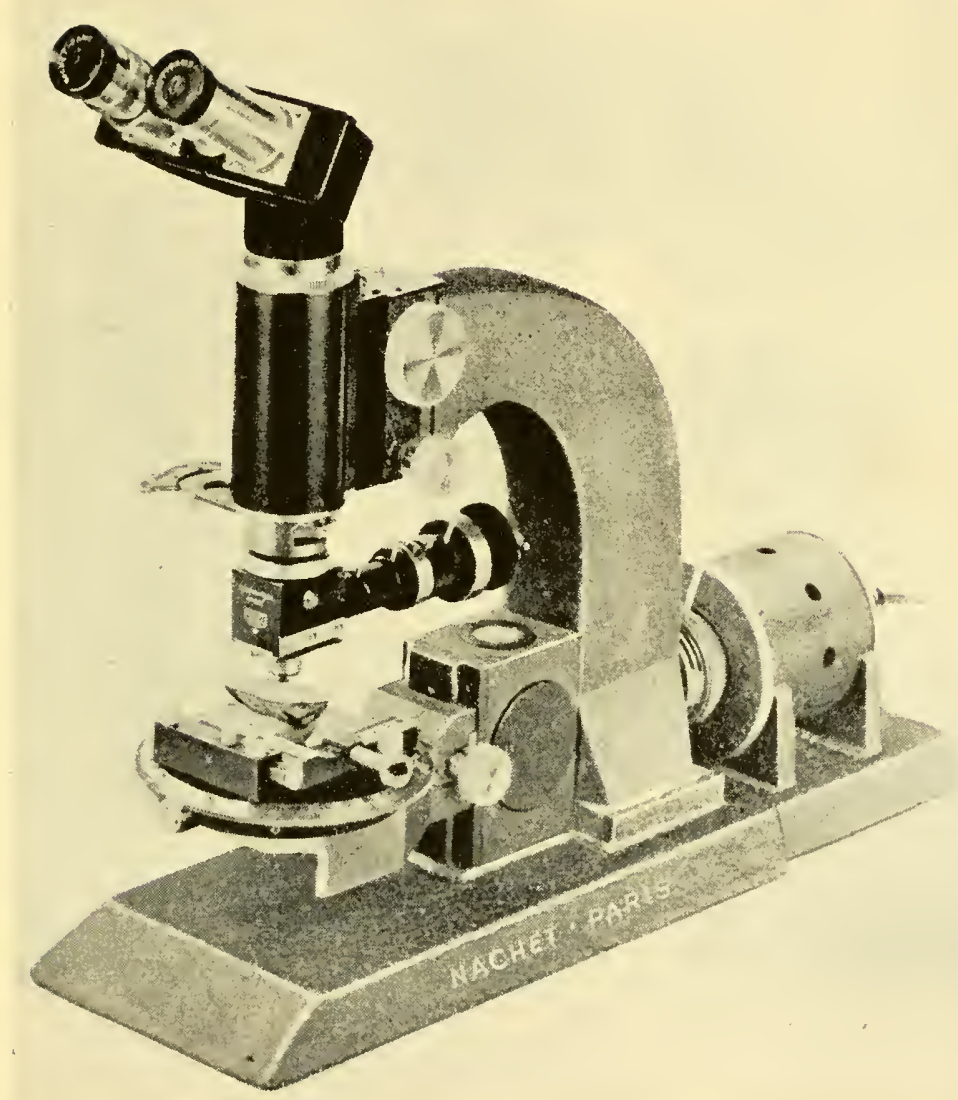

FIG. 4.31. Nomarski interference microscope (Nachet). 


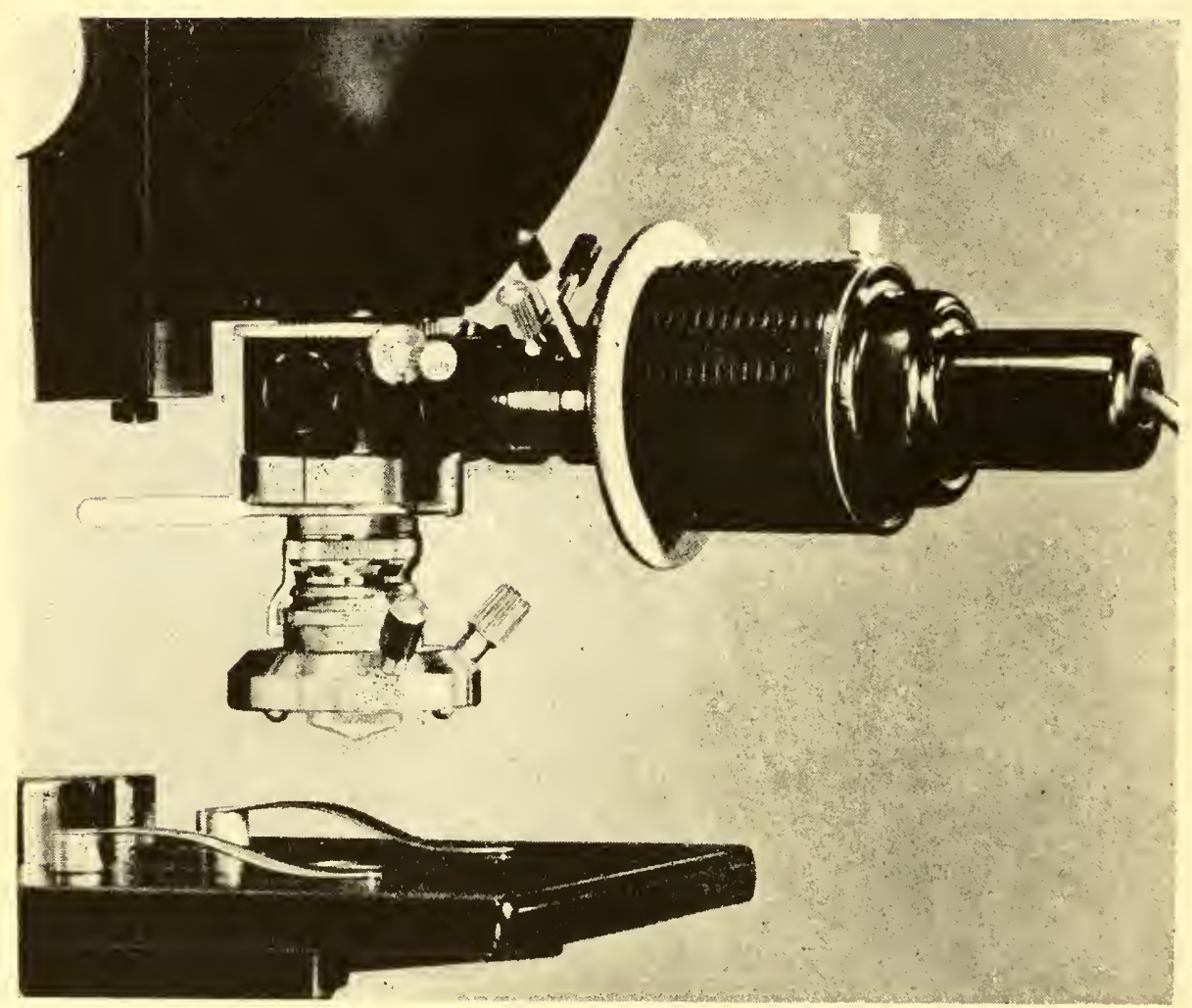

FIG. 4.32. Bausch and Lomb interference microscope. 
fore be applied to any objective or any magnifying power. On the other hand, the mirror $M$ is substituted for a semi-reflective plate thus allowing full aperture of the pupil.

\section{Multi-wave layouts}

The multi-beam interferometer is eminently suitable for investigating surface micro-structures. The procedure is to dress the surface so that it becomes highly reflective and to lay on it a metallized

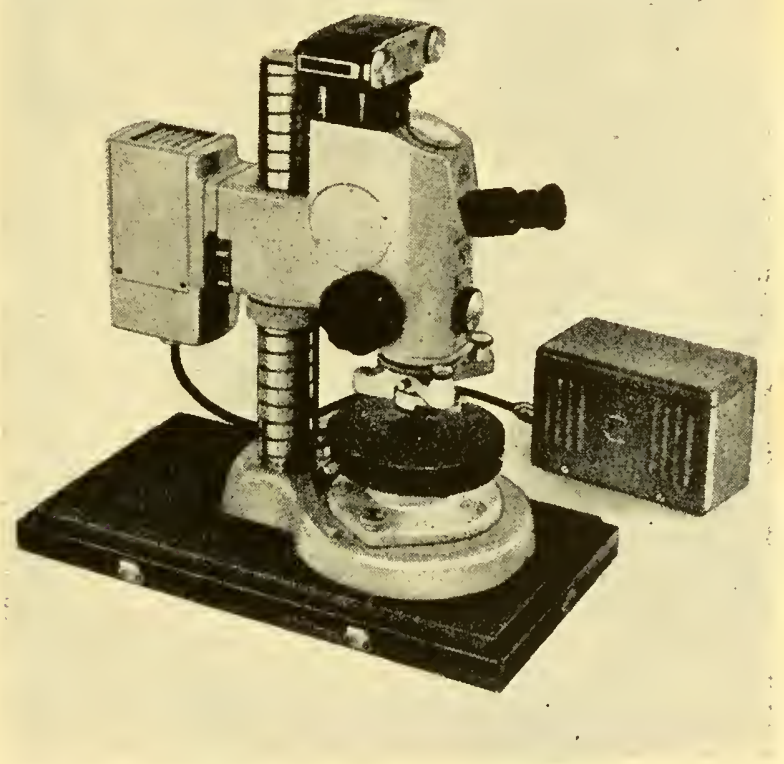

FIG. 4.33. "Multimi" Johansson interference microscope.

optically-flat surface. The numerous inter-surface reflections taking place evince a system of fine fringes. The process of this phenomenon is similar to the one described in Chapter III, $\S 10$. These fine fringes are tantamount to topographical contour lines and enable detection of very minute surface flatness imperfections in the object. Figure 4.32 shows a microscope constructed on this basic principle by Bausch and Lomb. Reichert, VEB Zeiss, Johansson (Fig. 4.33), Hilger and Watt (Fig. 4.34) have also built multi-wave interference microscopes. 


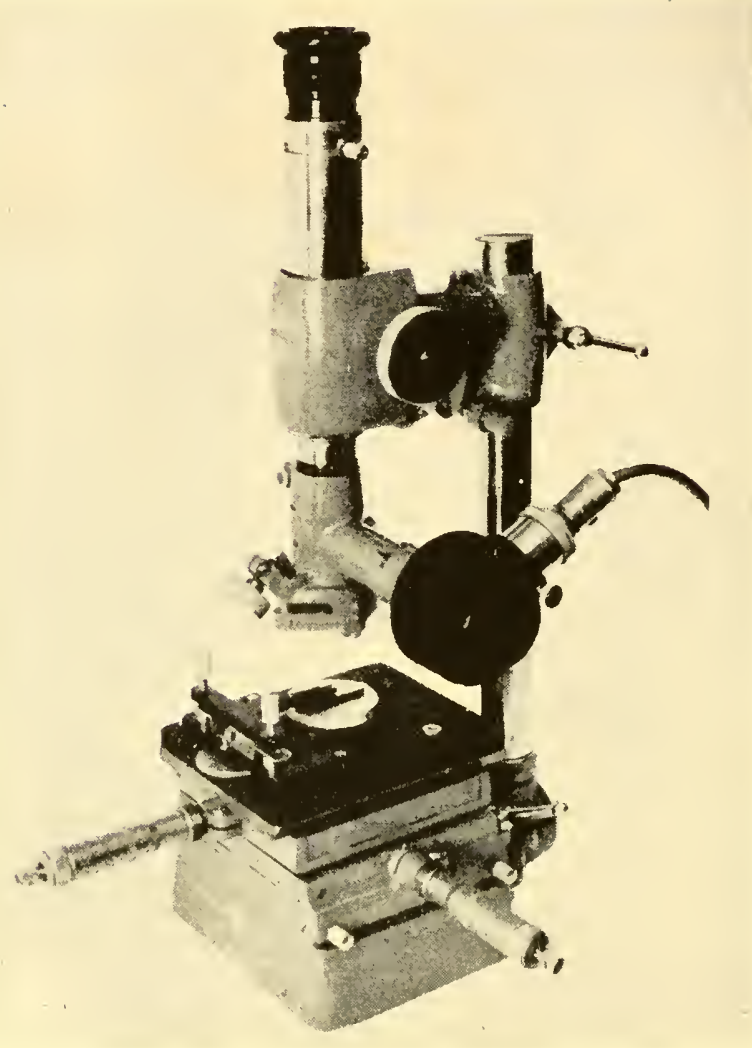

FIG. 4.34. Hilger and Watt interference microscope.

Application of reflecting interference microscopes to micro-hardness tests

Hardness of a material may be measured by the resistance it displays against penetration of another material to which a given amount of force is applied. In the Vickers method, the impressing element is a diamond square-based pyramid whose vertex angle is at $136^{\circ}$. Since the geometrical shape of the pyramidal impression is known. its area is determined by measuring the diagonal of the square base. Let $F$ be the force exerted against the impressing element (applied by a weight) and $S$ the impressed area. hardness $H$ is educed by:

$$
H:=\frac{F}{S} .
$$


Owing to the diamond's penetration, the surrounding surface is distorted. Such distortions are those which are investigated by means of interferometers. Figure 4.35 shows in the Zeiss interference mic-

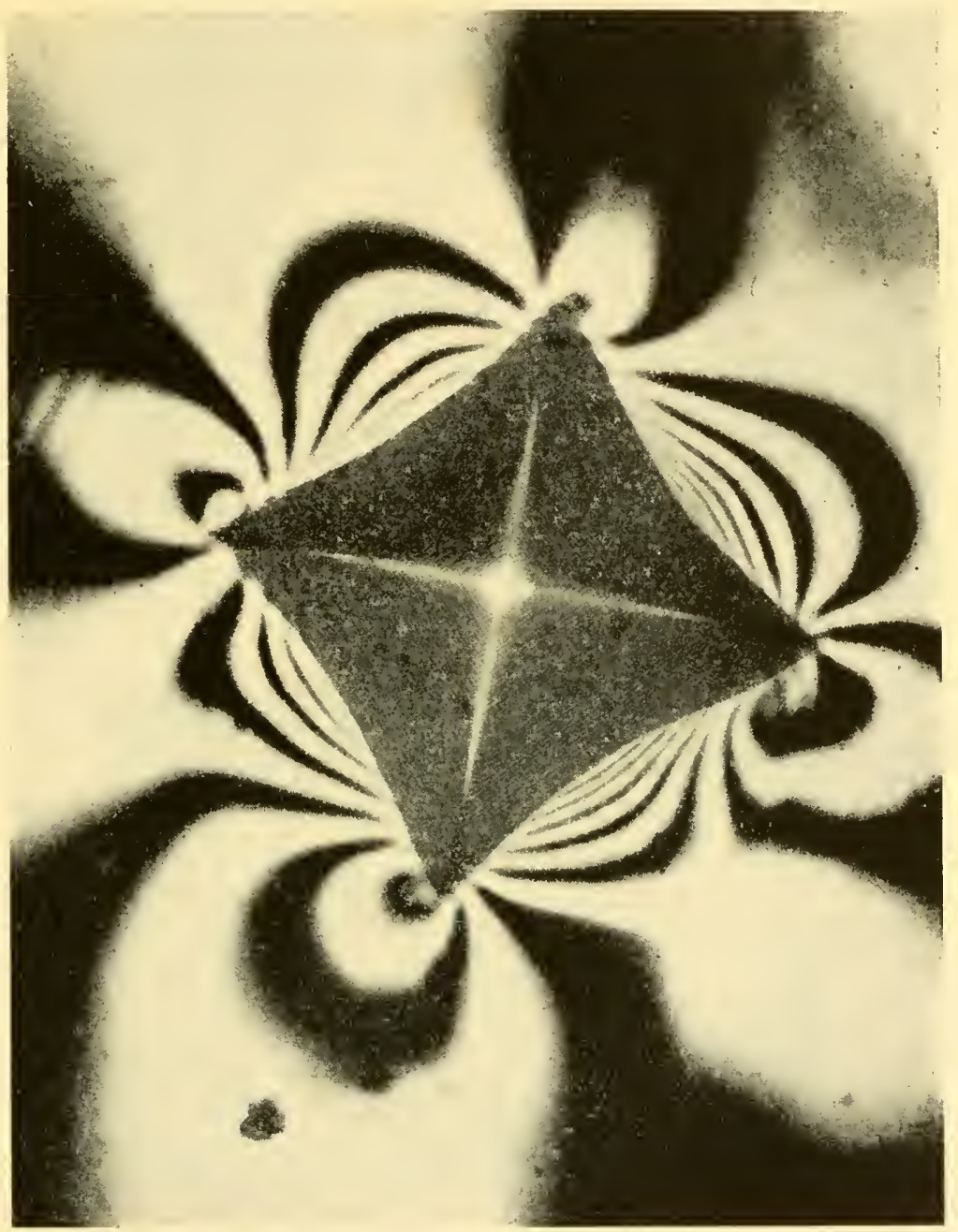

FiG. 4.35. Fringes brought about by the distortions surrounding the impression in microhardness testing (Zeiss interference microscope. Von Martin Uhling: courtesy of Zeiss).

roscope (photograph by Von Martin Uhling) ihe fringes brought about by the distortions surrounding the impression. Figure 4.36 shows the striking unsymmetrical impression obtained by Tolansky when 


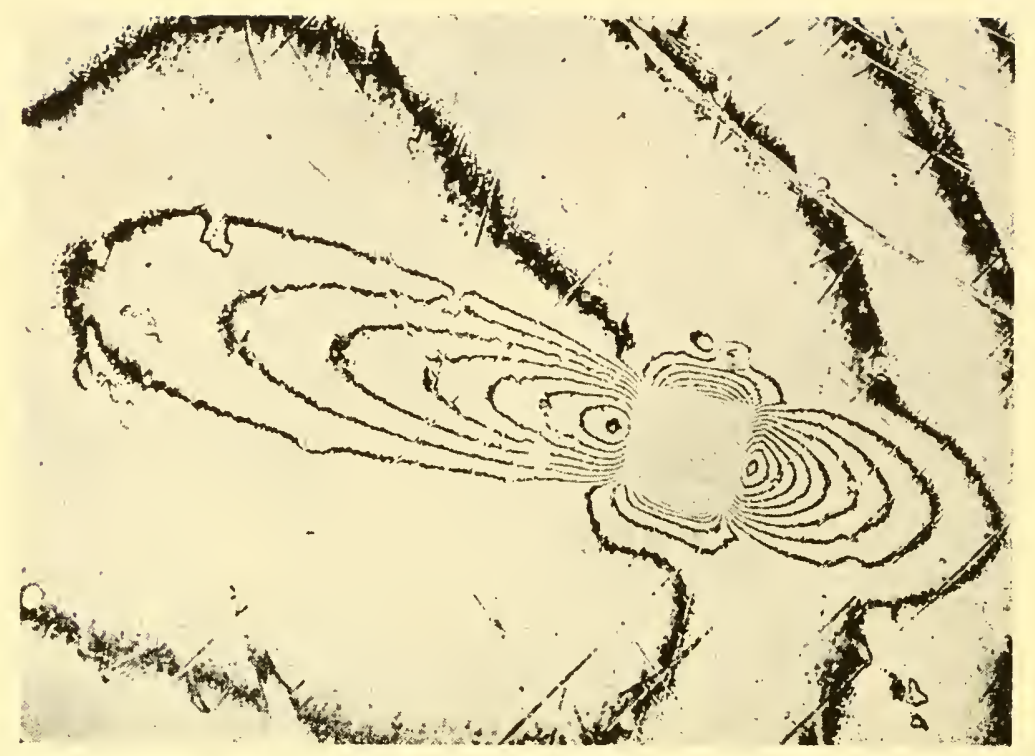

FIG. 4.36. Unsymmetrical impression obtained by Tolansky when carrying out microhardness tests on a tin mono-crystal (courtesy of Prof. Tolansky).

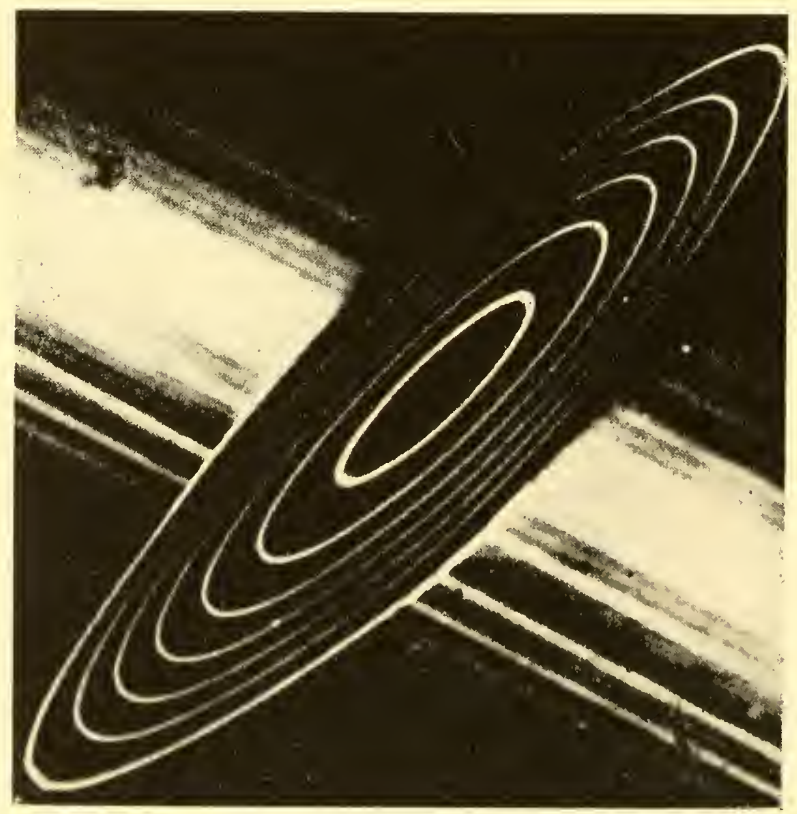

FlG. 4.37. Fringes when a small stecl diamond-chip wheel is applied against a surface itt a given pressure (courtesy of Prof. Tolansky). 
carrying out micro-hardness tests on a tin mono-crystal. Tolansky also investigated the anisotropic hardness of diamond when subjected to abrasion tests (Fig. 4.37). In such a test, a small steel diamond-chip wheel is applied against a surface at a given pressure. The speed of wheel rotation and the time the test lasts are also measured. The small shallow notches brought about by abrasion are observed; their interferometer-determined size show the degree of resistance to crushing and to wear, the material can withstand through polishing. Setting the grinding-wheel at the proper angle enables one to determine the specific resislance properties of the diamond in a given direction.

Investigation of surfaces whose thickness variations exceed one micron

When thickness variations exceed several microns, interpreting interference data becomes a tricky matter. Then the replica method may be applied, either to refleciive or non-reflective (diffusing) surfaces. A solution of plastic material is applied on the surface, which, after solidifying, forms a thin film. The latter is then removed and set on an aluminized reflective, flat optical plate. Let $R$ be the film (Fig. 4.38), a surface flatness imperfection of which, denoted by $M$, is of thickness $e$,

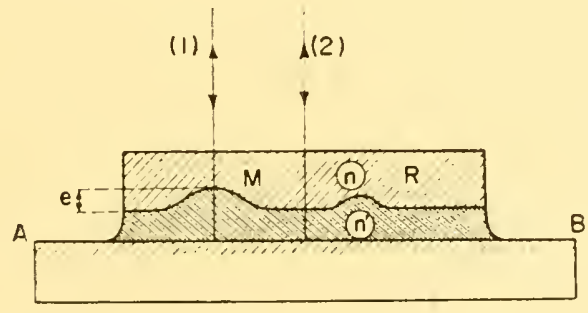

FIG. 4.38. The replica method.

and $A B$ the flat reflective surface. Let us insert between the film and the surface $A B$ a liquid whose index is $n^{\prime}$. If $n$ is the film index, the path difference between the ray (1) passing in the proiruding area $M$ and the ray (2) passing in a flat area, is:

$$
d=2\left(n-n^{\prime}\right) e .
$$

Path differences are doubled as the system is observed by reflection, e.g. through a Michelson-type interference microscope (Figs. 4.23, $4.24,4.26)$. Now let us assume that the path difference is correlated 
with a shift equating one fringe. The thickness $e$ is:

$$
e=\frac{i}{2\left(n-n^{\prime}\right)} \text {. }
$$

Therefore, selection of closely-related indices $n$ and $n^{\prime}$ provides a shift equating one fringe with a substantial thickness $e$. Let us take. e.g. $n-n^{\prime}=0 \cdot 1$. A shift equating one fringe in the interferometer corresponds to a thickness $e=5 \lambda$ (3 microns when $\lambda=0.6 \mu)$. Assuming the original surface itself to be observable, a $5 \%$ thickness would bring about a ten-fringe shift. Therefore, path differences can be reduced by artificial means to practical measurements and interpretation. Replicas can also be observed in transmitted-light interference microscopes. In this case the expression 4.2 becomes:

$$
d=\left(n-n^{\prime}\right) e \text {. }
$$

Since the light passes through the object only once, path differences are no longer doubled and the expression 4.3 becomes:

$$
e=\frac{i}{n-n^{\prime}} \cdot
$$

Retaining the same numerical example, a shift equating one haif of a fringe interspace is observed on the replica.

\section{OBSERVATION IN POLARIZED LIGHT}

Anisotropic metals and alloys are observable in polarized light. Addition of a polarizer and an analyser converts the reflected-light microscope into a polarizing one. The incident light being thus polarized, the light reflected by the surface is observed. After removing the analyser, rotating the sub-stage alters the direction of the surface which is thus observed in incident linear-polarized light. If the investigated substance is anisotropic, intensity changes occur during the observation. When studying birefringent transparent substances. two directions at right angles are considered, according to which a vibration propagates with two different indices. When an anisotropic polished surface is involved, there are two perpendicular directions correlated to two different reflectances. The greatest and the least reflection are achieved when the incident vibration is parallel to these two directions. known as "main directions". The investigated 
anisotropic surface, in many substances, shows a double reflection that varies as does the wave-length of the incident light. This is bireflective dispersion and characteristic colours are observed. To complement the resulting data the analyser is crossed with the polarizer. Rotating the sub-stage causes the anisotropic surface to exhibit 4 directions at right angles along which extinction still obtains. Intensity is highest at $45^{\circ}$ from such directions.

Let us now set the sub-stage so that the "main" directions lie $45^{\circ}$ from the incident-vibration direction. Owing to two different reflectances correlating the two main directions, the incident vibration rotates about a small angle. Let $O X$ and $O Y$ be the two main directions of the anisotropic surface and $O P$ the amplitude of the incident linear vibration (Fig. 4.39). OP may then be decomposed in the two vibrations $O X$ and $O Y$ directed according to the two main directions. After being reflected, the vibrations $O X$ and $O Y$ do not have the same amplitude since reflectances are always below unity. Owing to the difference between the reflectances correlating $O X$ and $O Y$.

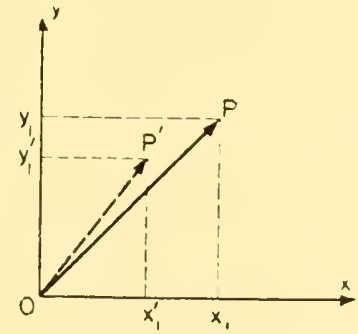

FIG. 4.39. After being reflected, the incident vibration $O P$ becomes $O P^{\prime}$.

the latter become $O X_{1}^{\prime}$ and $O Y_{1}^{\prime}$ after being reflected by the object. If no phase change occurs after the reflection, $O X_{1}^{\prime}$ and $O Y_{1}^{\prime}$ exhibit the linear vibration $O P^{\prime}$ which has rotated about a small angle in relation to $O P$. To every main direction corresponds not only a specific reflectance but a specific phase change as well. As phase changes are not the same for both main directions, the incident linear vibration is converted after being reflected, in an elliptic vibration. Generally, the ellipse is substantially flattened and its major axis differs but little from the direction of the incident vibration.

As the angle of rotation depends on the reflectance ratio of the main directions, the bi-reflective dispersion generates rotations which are not the same at all wave-lengths. Rotating the analyser 
(the polarizer and the sub-stage remaining stationary) will evince seriation a series of striking colours.

In the foregoing observations, the reflectance of the main directions, the colours, the ellipticity of the reflected vibration, the angle of rotation and so forth, have provided valuable data pertaining to polished metal surfaces. As already mentioned, the additional equipment required is simple: merely setting a polarizer in the vertical illuminator ahead of the $45^{\circ}$-slanted semi-reflective mirror and an analyser between the latter and the eye is all that is required. To avoid the birefringent superimposition effects, the objective's lenses are to be tension-free. The $45^{\circ}$-slanted mirror has its lower surface (on the objective side) coated with a semi-reflective layer so as to increase the reflectance. Conversely, the upper surface (eyepiece side) is processed to lower the reflectance thus preventing formation of

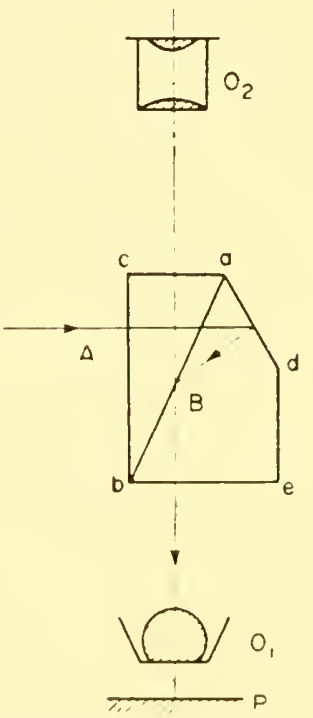

FIG. 4.40. Foster device (Bausch and Lomb).

a double image. Owing to the reflection of the $45^{\circ}$-slanted mirror, when using two crossed polarizers, extinction is not uniform within the whole field. In the Cooke, Troughton and Simms "Metalore" microscope, the $45^{\circ}$-slanted mirror is in parallel light and extinction is uniform within the whole ficld. The neat device, designed by Foster (Bausch and Lomb) serves as $45^{\circ}$-slanted plate and polarizing device at the same time (Fig. 4.40). A spar prism, consisting of the two 
cemented elements $A$ and $B$, is set in the illuminator. The light originated by the vertical illuminator is reflected by the ad face which acts as a mirror. The elements $A$ and $B$ are cemented with a substance whose index is such that only the ordinary ray is reflected by the surface $a b$. The extraordinary ray is transmitted and absorbed by the darkened face $b c$. After being reflected on the object $P$, the ordinary ray is again fully reflected by $a b$ and. if the object is not anisotropic, no light reaches the eyepiece $O_{2}$. The device works as would two perfectly-crossed polarizers. If the object is anisotropic, the same effects as described previously occur. To obtain the equivalent of an analyser rotation, a quarter-wave plate is inserted between the objective and the Forster prism. Owing to the double passage, the quarter-wave plate brings about the same effects as a half-wave plate and the incident vibration is rotated without being altered.

\section{SCRUTINIZING OF HOT-METAL SLRFACES}

Scrutinizing structure changes taking place at high temperatures, such as re-crystallization, are often desirable. Usually, to prevent oxidation, the sample is put in vacuo or in an actionless atmosphere. The chamber comprises a fused quatz optical peep-hole through which the sample is observed. To protect the microscope optical system,

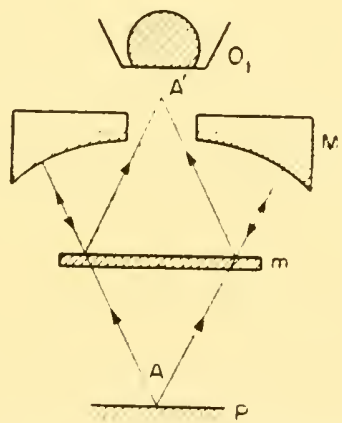

FIG. 4.41. Dyson long working distance attachment.

the chamber walls are cooled. The objective's working distance must be such as to allow focusing within the chamber. Owing to their long working distance, mirror-objectives are particularly suitable for this work (cf. Chapter I, §3). A spherical-surface objective, of numerical aperture $n \sin u=0.65$, provides a working distance extending 
over 14 millimetres. A standard objective may be converted into a long working-distance objective by using the Dyson layout (Fig. 4.41). The object $P$ lies in the centre of the curvature $A$ of the concave mirror $M$. The object-originated rays pass through the semi-reflective plate $m$ and are reflected back by the mirror $M$ on to the plate $m$ and end at $A^{\prime}$. Therefore, $A$ is imaged at $A^{\prime}$, i.e. the object, which may now be examined by a powerful objective with short working-distance. The working distance of the Dyson layout is approximately 13 millimeters. 


\section{CHAPTER V \\ Geometrical Measurements}

\section{SETTINGS IN MICROSCOPY}

MiCROSCOPE measurements are based on two types of settings: transverse and axial. The former entails no alteration in sharpness. Let us consider, for instance, a small object whose tips are properly focused. In microscopes comprising a micrometer eyepiece, the spiderline is shifted so as to align it seriatim with the imaged edges of the object. As the spider-line shift is shown by a micrometer screw, the width of the observed object can be readily measured. These operations entail two transverse settings. A transverse setting is made every time the spider-line of the eyepiece micrometer is brought in alignment with the imaged point of an object without altering the focusing and the spider-line is shifted transversely. The axial setting is a sharpness setting. For instance, it is required to locate the position in depth of a given point of the image: to do this, the fine adjustment is actuated until the point considered is sharp concurrently with the eyepiece reticle. Shifting the microscope axially brings the imaged, considered point, in coincidence with the eyepiece reticle. The object-point lies in a definite position in depth with respect to the microscope and is so located by an axial setting.

\section{SETTING ACCLRACY}

When the microscope is fitted with a $0.5 \mathrm{~mm}$ diameter exit pupil (optimum magnification) setting accuracy is practically restricted by the diffraction laws (cf. Chapter I, $\S 8$ ). Let us first investigate this case.

Transverse setting accuracy

Let $A$ be the focused object (Fig. 5.1). To effect a transverse set ting, the spider-line $R$ of the reticle is shifted in a front plane so as to be in coincidence with the image $A^{\prime}$ of $A$. Now, let us assume 
the object considered to be either a point or a line. Granting that such coincidence can be brought about. under normal conditions. with an accuracy attaining one hundredth of the diffraction disk diameter: the matter could be reasoned taking as basis the diffraction disk lying in the image plane $A$, and then reverting to the space-object.

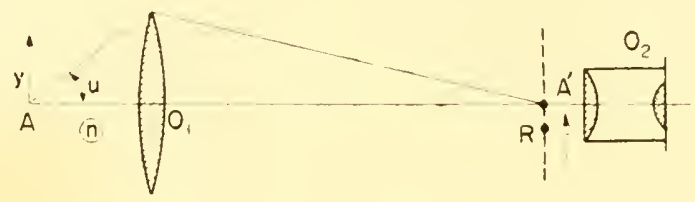

FlG. 5.1. Lateral setting.

dividing it by the objective's magnification. A prompter method is to consider direct the phenomena taking place in the plane of the object $A$.

Let $n \sin u$ be the objective's numerical aperture (N.A.) and i the wave-length. The diffraction-disk diameter in the object plane is $1 \cdot 22 \lambda / n \sin u$. Therefore, $A$ can be located by a transverse setting with an accuracy $1 \mathrm{y}$ equating:

$$
I y \geqslant \frac{1 \cdot 22 \%}{100 n \sin u} .
$$

i.e. accuracy within $0.02 \mu$ for $i=0.6 \mu$ and a N.A. $=0 \cdot 30$.

N.B. The foregoing implies, of course, a mechanically excellent and vibration-free microscope.

If the object is the edge of an area, accuracy is much impaired and will in no way be provided by the foregoing formula. Axial setting accuracy (Fig. 5.2)

The problem involved is the following: the object being properly focused at $A$, to what length $\varepsilon$ can it be shifted or-what amounts to the same thing-can the microscope be shifted. A remaining motionless, without the eye noting any change in sharpness? Now, if the object-point $A$ can be shifted to $A_{0}$ for a distance $\varepsilon$ without changing sharpness of the image. it can be shifted for the same amount and with the same result to the right at $A_{1}$. Under such conditions, the eye perceives no change in image sharpness. irrespective of the object's position within the interspace $A_{0} A_{1}=2 \varepsilon$. Such interspace determines 
the axial setting accuracy. It is not feasible to locate the position in depth of the object $A$ with greater accuracy than $A_{0} A_{\mathrm{L}}=2 \varepsilon$. Granting Lord Rayleigh's tolerance (Chapter I, $\$$ 6), defective focusing should not entail a variation of the optical path exceeding one quarterwave. Then, accuracy of axial setting is:

$$
\begin{gathered}
2 \varepsilon \geqslant \frac{\lambda}{4 n \sin ^{2} u / 2} \\
2 \varepsilon=1 \mu \quad \text { for } \quad \lambda=0.6 \mu \quad \text { and } n \sin u=0.65 .
\end{gathered}
$$

To sum up, provided the microscope be used with a $0.5 \mathrm{~mm}$ diameter exit pupil, accuracy of transverse and axial settings can be educed from the following formulae:

Transverse setting

$$
\Delta \geq \frac{1 \cdot 22 \%}{100 n \sin u}
$$

Axial setting

$$
2 \varepsilon \geqslant \frac{\lambda}{4 n \sin ^{2} u / 2}
$$

N.B. It should be pointed out that measurement accuracy depends on many factors other than setting accuracy, such as stability, clearances, expansion, stresses and so forth. It is but rarely that full use can be made of the setting accuracy, provided by high N.A. objectives.

Role of the observer's eye in axial settings

Let us consider an axial setting carried out with an instrument lacking a reticle. As focusing changes, the instrument-image changes in depth and the instinctive excursion of the eye keeps it in focus as long as it remains within the limits of sharp vision.

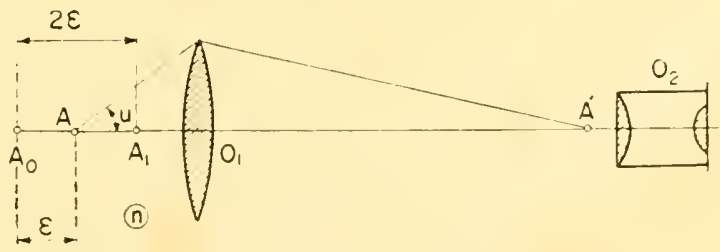

FIG. 5.2. Accuracy of an axial setting $(2 \varepsilon)$.

Setting on the image may therefore be correlated to any position of the image within the eye-accommodation amplitude. The result is some uncertainty as to the object's position, called "depth of accommodation". 
Let $d p$ be the depth of accommodation in metres, $G$ the microscope magnifying power, 250 millimetres the reading distance.

If $\pi$ is the accommodation amplitude in dioptries, $n$ the immersion index, then:

$$
d p=n\left(\frac{0 \cdot 25}{G}\right)^{2} \pi .
$$

Let $P$ be the distance to the near point and $R$ to the far point. These two distances, showing in metres the accommodation amplitude (in dioptries), is educed by:

$$
\pi=\frac{1}{P}-\frac{1}{R}
$$

If $\omega$ is the exit pupil diameter, the expression may be written thus:

$$
d p=\frac{\pi\left(v^{2}\right.}{4 n \sin ^{2} u}
$$

Hence, if the accommodation is to be reduced as far as is feasible, the exit pupil must be as small as possible, i.e. magnify to the utmost, for a given objective.

It may be required to ascertain the admissible eye-accommodation variation enabling one to obtain the accuracy shown theoretically by diffraction (equation 5.2): Let $x^{\prime}$ denote such variation. The resulting data are tabulated hereunder:

\begin{tabular}{l|c|c|c|c|c}
\hline o in $\mathrm{mm}$ & 0.2 & 0.25 & 0.50 & 0.6 & 1 \\
\hline$\tau$ in dioptries & 10 & 5 & 1.2 & 0.6 & 0.3 \\
\hline
\end{tabular}

Regardless of eye accommodation (the accommodation amplitude of a normal eye is 10 dioptries), a $0.2 \mathrm{~mm}$ exit pupil provides theoretical accuracy. Therefore, such an exit pupil does not require a reticle. It should be pointed out that such small exit pupils are not advocated (short focal-length eyepicces entail intricate design and prominent entoptic phenomena). If now, the eycpiece is provided with a reticle. it is assumed that a trained eye can be accommodated steadily with $0 \cdot 1$ dioptrie. Under such conditions, with an cyepiece comprising a reticle and in accordance with the foregoing table, exit pupils ex- 
ceeding $1 \mathrm{~mm}$ would be permissible. However, it is best not to exceed a $0.5 \mathrm{~mm}$ diameter exit pupil, corresponding to a one dioptrie admissible eye-accommodation variation, assuming the eye to be a perfect instrument.

\section{MEASURING A LENGTH IN A FOCUSING PLANE BY MEANS OF TWO TRANSVERSE SETTINGS}

The length to be measured in the focusing plane is shown (Fig. 5.3) by the $A_{1} A_{2}$ interspace. $A_{1}$ and $A_{2}$ are imaged at $A_{1}^{\prime}$ and $A_{2}^{\prime}$ in the plane observed through the micrometer eyepiece. The reticle is aligned

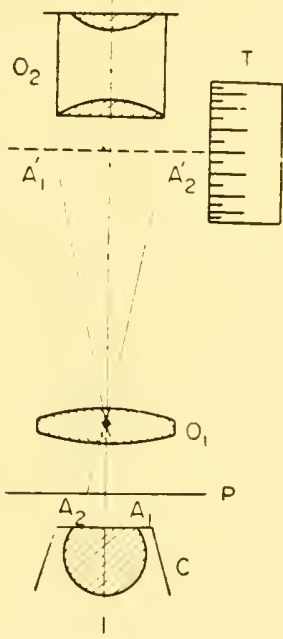

FIG. 5.3. Measuring a length by means of two lateral settings.

with $A_{1}^{\prime}$ and $A_{2}^{\prime}$ by means of the graduated drum $T$ that drives the spider-line transversely. Provided the graduation of $T$ is calibrated, the actual length $A_{1} A_{2}$ within the object is readily ascertained by means of the expression:

$$
A_{1} A_{2}=\frac{A_{1}^{\prime} A_{2}^{\prime}}{g}
$$

where $g$ is the magnification of the objective $O_{1}$. Such calibration is achieved by means of an objective micrometer and allows the lengths $A_{1} A_{2}$ to be read off the drum $T$ : the specimen is substituted for the objective micrometer comprising $n$ divisions per millimeter and the 
number $N$ of divisions of the drum $T$, tallying with $n^{\prime}$ divisions of the objective micrometer, is noted. Every division on the drum $T$ equates therefore $n^{\prime} / N n$ millimeters. The length to be measured is assumed to be minute and in the field centre.

\section{MEASURING A THIN OBJECT BY TWO AXIAL SETTINGS}

Thin objects may be measured through a conventional microscope by means of a previously-calibrated fine-focusing adjustment. The screw of the latter usually comprises a datum-mark which is moved opposite the graduation. Such graduation can be calibrated as follows: let us use as calibration reference an optically-tested cover-glass the thickness $e$ of which is ascertained. This cover-glass is semi-aluminized on both faces, each of which has an optical density approaching unity, i.e. 2 in the aggregate, approximately. Therefore, the cover will only let $1 / 100$ of the incident light pass through, which is adequate. Next, both faces are scratched. These scratches, plainly visible in a suitable contrast, serve as reference-marks to set the object-cover faces. Thereupon the cover-slip is set in the microscope stage (the cover-slip being secured to the object slide, if required) and both faces of the coverglass are sighted seriatim by means of the fine adjustment, using the scratches as reference-marks. Since $p$ divisions of the fine-adjustment screw correspond to the thickness $e$ in millimetres of the cover-glass, a one-division shift of the screw equates an $e / p$ thickness (in $\mathrm{mm}$ ) in a medium of index $n$, index of the cover-slip, and would correlate an $e / n p$ shift (in $\mathrm{mm}$ ) in air and $n^{\prime} e / n p$ (in $\mathrm{mm}$ ) in a medium of $n^{\prime}$ index.

In order to obtain the actual length, the vertical length comprised in a medium of index $n^{\prime}$ is to be multiplied by $n^{\prime}$. The presence of a cover-glass having a different index does not alter measurements.

\section{MEASURING A LENGTH WHOSE ENDS DO NOT LIE IN THE SAME FOCUSING PLANE}

The length to be measured is $A_{1} A_{2}$ (Fig. 5.4) but, as neither $A_{1}$ nor $A_{2}$ lie in the same front plane, focusing must be altered when changing over from $A_{1}$ to $A_{2}$. Once $A_{2}$ is focused, the location of the image $A_{2}^{\prime}$ is ascertained by means of the eyepiece micrometer spider-web. Next, in the case shown in Fig. 5.4, in order to focus $A_{1}$, the microscope is tilted upwards slightly to the extent of $\varepsilon$. Things work out as if $A_{2}$ remained in focus and that the setting on $B_{1}$ (projection of $A_{1}$ ), was 
made through the front plane along $A_{2}$. The two transverse settings yield the length $A_{2} B_{1}$ and the two axial ones the distance $\varepsilon$. The actual line $A_{1} B_{2}$ then equates $1\left(A_{2} B_{1}^{2}+\varepsilon^{2}\right)$. If the length $A_{1} A_{2}$ is encompassed

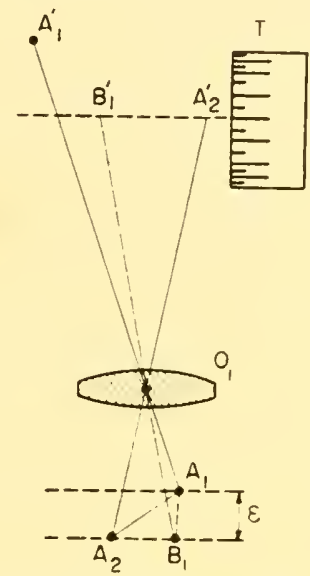

FlG. 5.4. Measuring a length by means of lateral and axial settings.

in a medium of index $n, \varepsilon$ is to be multiplied by $n$. In such a measurement, the microscope optical axis is not to be shifted and, in a series of measurements, the dispersion in performance data provides the limits of accuracy.

\section{MEASURING SMALL AREAS AND VOLUMES}

Compounding the measurements described in $\$ 3.4$ and 5 evinces the data required. Surfaces may also be measured by means of an integrating stage or an integrating eyepiece. This is tantamount to cutting up the object in surface elements and simply adding them up by means of the integrating stage.

\section{DETERMINING THE NORMAL IN A POINT OF A SMALL REFLECTING SURFACE}

The standard microscope eyepiece is to be substituted for one of special design (Fig. 5.5). The aperture of screen $E$ is illuminated by the lamp $L$. The light, originated from $T$ is reflected by the $45^{\circ}$-slanted semi-reflective mirror $G$. The microscope is adjusted so as to image $T$ in the investigated surface $S$. $A$ is imaged in the area whose orientation is sought. After being reflected back from the surface $S$ on 
to the objective which images $A$, i.e. the aperture $T$, at $A^{\prime}$. Let us set at $A^{\prime}$ a reticle, symmetrical with $T$ in relation to $G$. The image of $T$ and the surface fully coincide at $A$ when the image of $T$ being reflected on $S$ is in complete coincidence with the reticle at $A^{\prime}$. There-

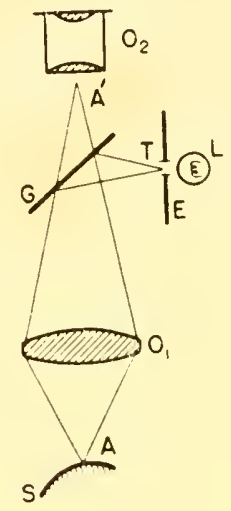

FIG. 5.5. Microscope using an eyepiece with illuminated reticle.

fore, a setting may be made on any element of the surface $S$. Now, its normal is to be located. The reticle $R$ is set in the focal plane of the objective $O_{1}$, the reticle being in the microscope's optical axis (Fig. 5.6). If the plane, tangent to the surface $S$ at the point $A$, where

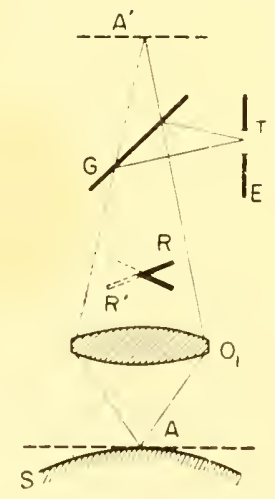

FIG. 5.6. Determining the normal in a point of a small reflecting surface.

$T$ is imaged, is perpendicular to the microscope's optical axis, the reflccted beam merges with the incident beam. To ascertain that such is the case, the eyepiece $O_{2}$ is removed and the eye at $A^{\prime}$ is placed 
on the reflected image of the aperture $T$ (the micrometer set at $A^{\prime}$ being removed together with $O_{2}$ ). Once eye accommodation is achieved in the focal plane of the objective $O_{1}$, the field is perceived brightly illuminated and bounded by the objective's surface. If the plane tangent to $A$ at the surface $S$ is perpendicular to the microscope's optical axis, it is evinced that the tip of the reticle $R$ is in alignment with its image $R^{\prime}$ (Fig. 5.7): the reason being that the rays, issuing

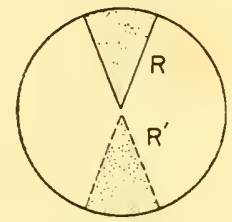

FiG. 5.7. Field aspect if the surface $S$ is normal to the "microscope"axis.

from $R$, are reflected on the small and virtually plane portion of $S$ which is illuminated (image of the aperture $T$ ). They are reflected back symmetrically and show a reversed image of the reticle $R$ in the focal plane of $O_{1}$. If the surface is not perpendicular to the microscope's optical axis at $A$, the reflected luminous cone rotates about $A$, the imaged reticle $R^{\prime}$ shifting at the same time (Fig. 5.8). Then, adding suitable elements to the microscope, enables one to carry out measurements of flat-surface angles. It should be pointed out that with re-

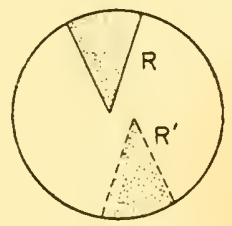

Fig. 5.8. Field aspect if the surface $S$ is not normal to the microscope axis.

ference to the reticle $R$ and its image $R^{\prime}$, all rays are reflected in a small area surrounding $A$. From the diffraction standpoint, the process taking place for $R$ and $R^{\prime}$ is as if the objective $O_{1}$ had the same diameter as the imaged aperture $T$ at $A$. The smaller the image, the poorer the accuracy. Using an area of $5 \mu$ on a $0.7 \mathrm{~mm}$ diameter cylinder (such as a needle) the direction of the perpendicular can be determined within $15^{\prime \prime}$. If the area has a $1 \mu$ diameter, accuracy is within $40^{\prime \prime}$. 


\section{HIGH PRECISION-MEASUREMENT MICROSCOPES}

The first microscope providing high-precision measurements were developed by C. Cantu and A. Ranzini and constructed by Messrs. Koristka. This instrument, designed to investigate nuclear plates, is shown in Fig. 5.9 (type MS2). The stage features two rectangular motions. The $x$ motion is obtained by means of a plate attachment so secured as to virtually preclude any $y$ motion ( $\mathrm{sag} .=0 \cdot 3 \mu$ for a $50 \mathrm{~mm}$ travel).

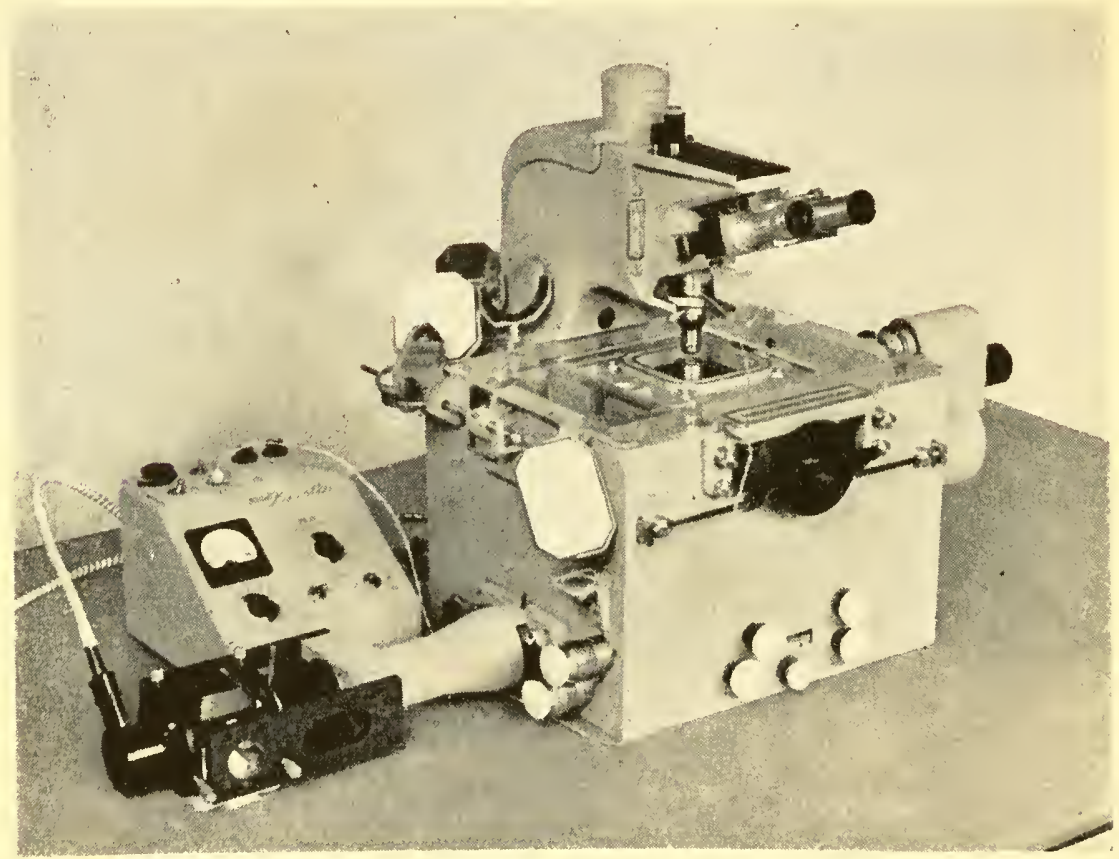

FIG. 5.9. Koristka high precision-measurement microscope.

The $y$ motion is conventional ("vee"-ways). Shifting the objective provides the $=$ motion which is obtained by a plate attachment and hydraulic drive. Prior to effecting measurements in order to determine the correction required, the $x, y$ and $z$ motions are tested by means of an interferometer.

The type MS2 was followed by the type R4, designed from investigating large plates, measuring 300 by 300 millimetres; the R4-type is particularly suited to speedy observations. In both types, the system used to carry out the transverse setting is ingenious: the eyepiece reticle is fixed. The image is slightly shifted by a parallel-sided plate 
which may be tilted (Ochiallini). This device does away with errors originated from micrometer screws. To the observation system is adjoined a reading device, next to the eyepiece, in which the micrometer scale is also shifted by a parallel-sided plate. Provided the thickness of the second plate exceeds that of the eyepiece and for an identical rotation of both plates, the micrometer motion is more extensive and measurements more accurate.

An electrical measuring and recording device may be added to this device. Rotations of the eyepiece plate take effect direct on an electrical system that feeds them to an electron computer. Highprecision microscopes are now built by several large concerns. These instruments, currently used in nuclear research, should likewise, prove very useful to biologists. 


\section{Measuring Refraction Indices of Transparent Objects: Unpolarized Two-wave Interference Microscopes}

Two wave interference instruments provide measurement of the path difference between two waves that travelled along different optical paths. The result is therefore always an optical path which is the product of a length multiplied by an index.

When the object involved is transparent - of thickness $e$ and index $n$ - and immersed in a medium of index $n^{\prime}$ the interference instrument shows $\left(n^{\prime}-n\right) e$. Provided the thickness $e$ and the index $n^{\prime}$ of the medium in which the object is immersed are known. the measurement evinces the index $n$ of the object. The interference microscopes shown in Figs. 3.4, and 3.8 are suitable.

\section{MEASURING PATH DIFFERENCES}

Let us consider the diagrammatic object in Fig. 6.1. It consists of a small rectangular parallelepiped the cross-section of which is shown at $B C B^{\prime} C^{\prime}$. Required to measure its index $n$, considering that it is

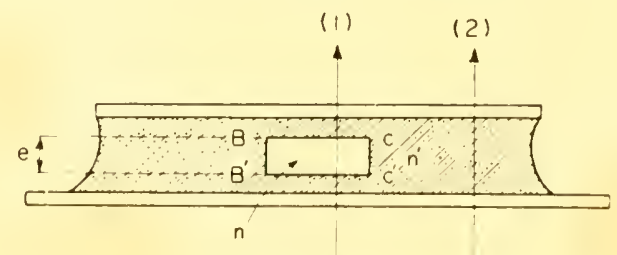

Fig. 6.1. Diagrammatic transparent object $B C B^{\prime} C^{\prime}$.

immersed in a medium of index $n^{\prime}$. The specimen is illuminated in monochromatic light of wave-length $\lambda$. Thickness of the object being $e$ and its index $n$, the optical path travelled by the ray (1) passing through 
the object is ne. A random ray (2), passing next to it, follows the path $n^{\prime} e$.

Over and under the horizontal lines $B C$ and $B^{\prime} C^{\prime}$, respectively, optical paths are identical for the rays (1) and (2) and do not intervene. The path difference between (1) and (2) is then:

$$
\delta=\left(n^{\prime}-n\right) e .
$$

This is the path difference brought about by the object.

The following may apply to any 2-wave interference microscope but, in order to simplify matters, the matter is set forth on the basis of Dyson's interference microscope (Fig. 3.4). Let us consider the rays, paralleling the ray $M A C$, corresponding to a wave surface which, after passing through the object, has the shape shown in Fig. 6.2.

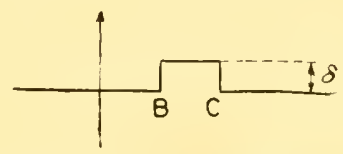

FlG. 6.2. The wave surface after passing through the object.

The rays paralleling the ray $B N$, which have not passed through the object, exhibit a flat surface wave. These two waves meet at $A^{\prime}$ where they interfere: their path difference 1 depends on the microscope adjustment (Fig. 6.3). In fact, the plates $L_{1}$ and $L_{2}$ are not parallelsided plates but small-angled prisms. Hence, the path difference varies continuously from one end to the other of the field of view. If the variation attains several wave-lengths. straight and parallel fringes are

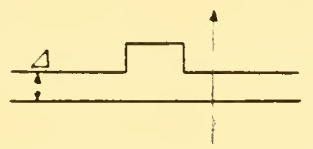

FIG. 6.3. The two wave surfaces in the image $A^{\prime}$.

developed, as shown in Fig. 6.4. The distance of both waves, i.e. their path difference $J$, varies: every time $J$ increases or decreases by $\lambda$. a shift is made from one fringe to another. Assuming that there are three fringes, the field aspect is shown in Fig. 6.5. The object lies in the area $A^{\prime}$. It is assumed that, in the area (2) (Figs. 6.4 and 6.5), the path difference $J$ between the two waves is an odd number of times $\lambda / 2$, e.g. $5 \lambda / 2$. Therefore, there is a dark fringe in area (2). If $\rfloor=7 \lambda / 2$ in (1) 
there will be another dark fringe in the area (1) surrounding the object. The path difference is $\lambda+\delta$ in the object-occupied area and differs from 1 . Therefore, the fringes within the object do not extend into the fringes lying in the field surrounding the object and a shift $\delta^{\prime}$ is observed. The number of fringes visible in the field of view depends

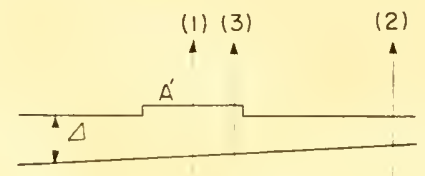

FIG. 6.4. The two wave surfaces when fringes are developed.

on adjustments. Fringe spacing is least when the slopes of both prisms dip toward opposite directions. The plate $L_{1}$ is shifted at right angles to the optical axis, without altering its orientation, by means of the screw $V$. This adjustment shifts the fringes in parallel relation without altering their spacing. Therefore, the path difference between the two waves shown in Fig. 6.4 may be varied. These waves move, retaining

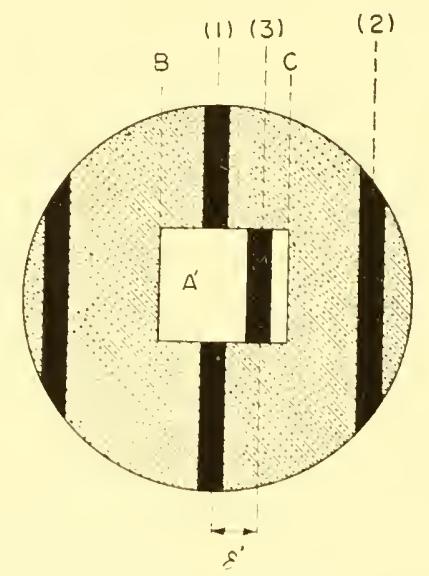

FiG. 6.5. Path difference measurement $\left(\delta^{\prime}\right)$.

their parallel relation, without affecting their relative slope. Let us assume that the object-originated path difference be less than $\lambda / 2$. The shift $\gamma^{\prime}$ is then the actual path difference and merges into $\delta$. In Fig. 6.5, $\delta$ is of the order of $\lambda / 3$. The shift is to be located in relation to the fringe (1) and not to fringe (2) since $\delta<\lambda / 2$. Yet, the path object-originated difference $\delta$ is not necessarily shown by the observed 
shift $\delta^{\prime}$. For instance, when $\delta=3 \lambda+\lambda / 3$, Fig. 6.5 is still the same although, this time, $\delta^{\prime}=\lambda / 3$ differs from $\delta$. In monochromatic light, all fringes are identical and a shift equating a whole-number times $\lambda$ cannot be observed for the figures are superimposed.

To obviate this difficulty, phenomena are observed in white light, this requiring that the dispersion of the object and its surrounding medium be not too extensive. Now, the instrument is adjusted to bring the white fringe, corresponding to $\Delta=0$ in the position (1), shown in Fig. 6.5. (Refer to Newton's colour scale, Chapter VII, § 1.) Next, actuation of the screw $V$ (Fig. 3.4) displays the fringes seriatim so as to bring the same fringe in the object. In order to make no error on the integral part of the path difference $\delta$ ( $3 \hat{\gamma}$ in the previous example) all that is required is to evaluate the so-measured rotation of the screw $V$ as $\lambda$-wave-length fringe intervals. Were the white fringe perceived, in the field of view, both in and out of the object, all that is needed is to evaluate the distance of these two fringes as mono-

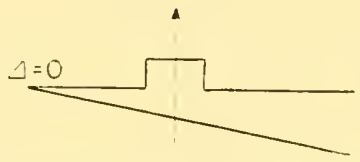

FIG. 6.6. The path difference 1 increases from left to right.

chromatically-spaced fringes. The measurement of the path-difference fractional part $\delta$, i.e. $\delta^{\prime}$ ( $\lambda / 3$ in the foregoing example), is accurately carried out in monochromatic light. To determine which of the two indices, $n$ or $n^{\prime}$, is the higher, is evinced by merely watching the motion of the white fringe $I=0$ during rotation of the screw $V$. Now the

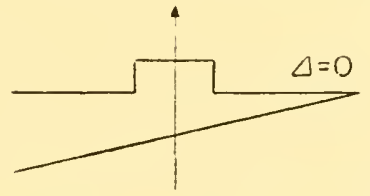

FIG. 6.7. The path difference 1 decreases from left to right.

wave arrangement in a given position of the screw $V$ defines the wave arrangement, i.e. whether the path difference $\perp$ increases from left to right, when the reference wave is below the wave that passed through the object, conversely, decreases (Fig. 6.7). This is ascertained by observing an object whose index is known. Let us bring the white 
fringe $\Delta=0$ in the object. If it is perceived outside the object to the left from the field when $n<n^{\prime}$, the arrangement is that shown in Fig. 6.8 and when $n>n^{\prime}$ in Fig. 6.9.

Let us assume that we are now dealing with the arrangement shown in Fig. 6.7, in relation to a specific position of the screw $V$. Let us

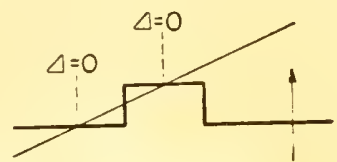

FiG. 6.8. Arrangement when $n<n^{\prime}$.

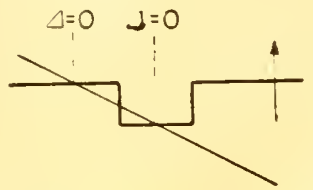

FiG. 6.9. Arrangement when $n=\cdot n^{\prime}$.

adjust the instrument so that the white fringe $\perp=0$ is at (1) (in Fig. 6.5), i.e. next to the object (Fig. 6.10). If, when rotating the screw $V$, the white fringe is brought in the object (Fig. 6.8), it is found that the white fringe outside it is shifting leftwards then, $n<n^{\prime}$.

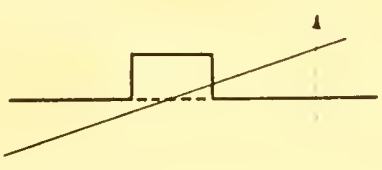

FIG. 6.10. The white fringe $1=0$ is outside the object according to Fig. 6.5.

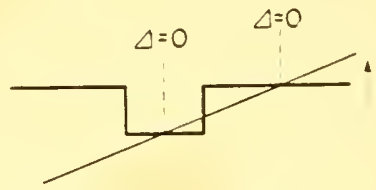

FIG. 6.11. The white fringe $I=0$ is in the object and outside it is shifted to the right.

If shifted to the right, $n>n^{\prime}$ (Fig. 6.11). If the object tapers edgewise there is no need to change over to white light. Let us substitute the diagrammatic object shown in Fig. 6.1 for the one in Fig. 6.12. It can be said that the path difference varies virtually continuously when

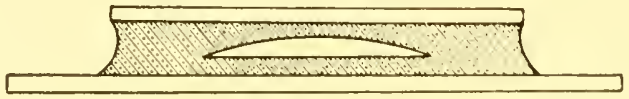

FIG. 6.12. Diagrammatic object for which the path difference varies virtually continuousl! when changing over from the medium surrounding the object to the object itself.

changing over from the medium surrounding the object to the object itself. There is, therefore, no break in the fringes as in Fig. 6.5. Fringes are continuously distorted. A specific fringe can be observed and its shife in the object definitely measured. The appearance of fringes is shown in Fig. 6.13 wherein the object has a random shape. The fringe 
$a_{0} b_{0}$ in the object corresponds to the fringe $a b$ outside it. The shift $\delta$ observed is the actual shifi and shows the path difference at the point of the object being considered. Likewise, to determine whether $n>n^{\prime}$ or $n<n^{\prime}$, all that is needed is to observe in monochromatic light an

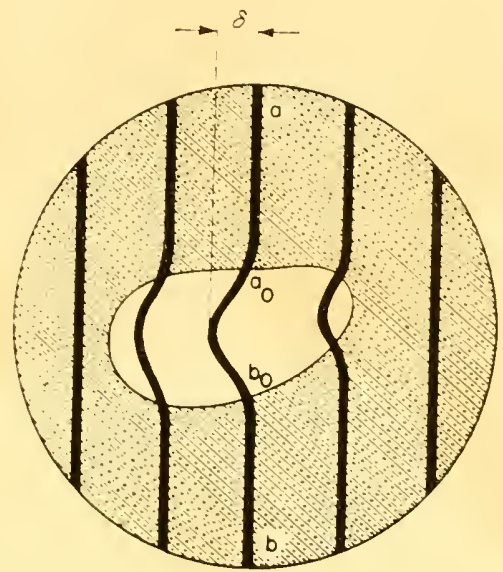

FIG. 6.13. For the object of Fig. 6.12, there is no break in the fringes. Fringes are continuously distorted.

object whose index is known, and which enables to follow up fringe distortions. The matter is readily solved by comparing the shift direction of the fringes in both instances.

\section{DETERMINING THE REFRACTION INDEX FROM PATH DIFFERENCE MEASUREMENT}

The foregoing measurements show $\delta=\left(n^{\prime}-n\right) e$. If $e$ and $n^{\prime}$ are known, $n$ is educed as follows:

$$
n=n^{\prime}-\frac{\delta}{e}
$$

Let us assume that the difference $n^{\prime}-n$ correlates a $\delta$ value of approximately $\lambda / 2$ and the $\delta$ error does not exceed $\lambda / 40$. If $e=20 \mu$ and assuming the measurement accuracy to be $1 \mu$, the relative error in the difference $n-n^{\prime}$ is:

$$
\frac{1\left(n^{\prime}-n\right)}{n^{\prime}-n}=\frac{4 \delta}{\delta}+\frac{1 e}{e}=0 \cdot 1
$$


Since $\delta \simeq \lambda / 2$ and $e=20 \mu$, then, $n^{\prime}-n \simeq 0.01$ and

$$
\Delta\left(n^{\prime}-n\right) \simeq 0.001 \text {. }
$$

In the example selected, the absolute error $\mathbf{I} n$ is approximately one unit to three places of decimals.

Accuracy can be greater but, as will be seen later (Chapter VIII, $\S 2$ ), a prominent factor, the incident light N.A., takes effect in measurement accuracy. 


\section{CHAPTER VII}

\section{Measuring Refraction Indices of Transparent Objects: Polarizing Interference Microscopes}

\section{APPLICATION OF THE FULL-DUPLICATION METHOD}

FULL-DUPLICATION polarizing interferometers are eminently suitable for microscope measurement of the refraction indices of transparent isotropic objects. As in all interference processes, measurements are based on the previous determination of path differences. Two procedures may be used:

(a) In the flat-tints method, the object and adjacent area exhibit two uniform but different tints. The path difference between the lightrays travelling through the object and those passing next to it can readily be calculated by means of Newton's scale of colours and the refraction index of the object educed therefrom. This process however, only approximates the index value.

(b) In the method using interference fringes in monochromaticlight, the whole field of view is streaked with straight, parallel and equidistant fringes. The fringes located in the imaged object are not in alignment with the other fringes. The shift, reckoned as interfringe spaces, shows the path difference between the rays travelling through the object and those propagating next to it. As previously, the refraction index is educed from the shift. The potential accuracy of the method is very high and depends on the precision with which fringe settings are carried out.

\section{MEASURING PATH DIFFERENCES BY MEANS OF THE FLAT-TINTS PROCESS. FULL-DUPLICATION PROCESS}

The following processes are applicable to objects of any shape but, in order to simplify matters, the diagrammatic object in Fig. 6.1 is again referred to. The object, whose refraction-index $n$ is required, 
is the small parallelepiped object cross-sectioned at $B C B^{\prime} C^{\prime}$, shown in Fig. 6.1. Measurements are based on tint discrimination according to Newton's scale of colours. Interference colours are, in fact, virtually the same for a substance of any index: the optical path ne depends, as the $n$ index, on the wave-length $i$. But, whereas $i$ varies by a factor of two throughout the visible spectrum. $n$ does not vary by more than 1 to 2 per cent. This method only yields an approximation since Newton's scale disregards the $n$ variations in relation to $\lambda$. Nevertheless, the method is expounded herein as it helps in grasping the phenomena described later on.

The microscope is illuminated in white light - it is assumed that dispersions in the media $n$ and $n^{\prime}$ do not differ overmuch. Before travelling through the object, the wave-surface is plane. After, provided $n<n^{\prime}$, it has the shape shown in Fig. 6.2. The arrow shows the direction of the light. Let us use a full-duplication polarizing interference microscope (Chapter III, $\S 5$ ). The microscope birefringent sysiem duplicates the incident wave and there are now two waves, $\Sigma_{1}$ and $\Sigma_{2}$, in the image plane observed in the eyepiece (Fig. 7.1). This arrangement, corresponding to $n^{\prime}>n$, is not the only one feasible. When $n^{\prime}<n$, the resulting arrangement is either the one shown in Fig. 7.2 or 7.3 , according as to whether the object-originated path

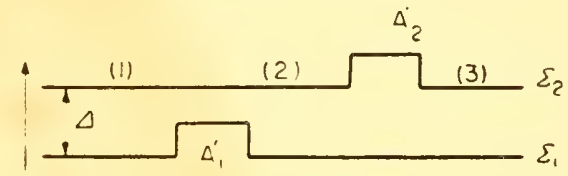

FiG. 7.1. Duplication of the transmitted wave $\left(n<n^{\prime}\right)$.

difference is greater than the one between the wave $\Sigma_{1}$ and $\Sigma_{2}$. For the moment, let us consider Fig. 7.1. In the areas (1), (2), (3) the distance between the two waves, i.e. their path difference, is brought about by the birefringent system. Polarizing interference microscopes include elements enabling one to adjust, as required, the path difference between the two waves $\Sigma_{1}$ and $\Sigma_{2}$ in the areas where they are flat (no object being present). For instance, in the microscope shown in Fig. 3.20, such adjustment is provided by merely shifting the Wollaston prism $W_{2}$ in a parallel direction and at right angles to the microscope axis.

Let us assume that the path difference equates $\Delta$ in the areas (1), (2), (3). The object thickness and index being denoted by $c$ and $n$, 
respectively, we have seen (Chapter VI, $\$ 1$ ) that the object-originated path difference, i.e. the path difference between the ray (1) that passes through the object and the ray (2) passing next to it, is:

$$
\delta=\left(n^{\prime}-n\right) e .
$$

However, the interference microscope brings in the path difference $J$ (adjustable as required) between the two waves $\Sigma_{1}$ and $\Sigma_{2}$ in the

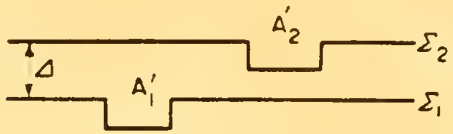

FIG. 7.2. Duplication of the transmitted wave $\left(n>n^{\prime}\right)$.

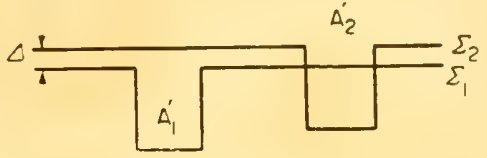

FIG. 7.3. Duplication of the transmitted wave $\left(1<\delta\right.$ and $\left.n>n^{\prime}\right)$.

plane areas where there is no object (Fig. 7.1). Hence, the path differences at $A_{1}^{\prime}$ and $A_{2}^{\prime}$ between the two waves $\Sigma_{1}$ and $\Sigma_{2}$, shall be (in the case of Fig. 7.1)
at $A_{1}^{\prime}$
$\Delta-\delta=\Delta-\left(n^{\prime}-n\right) e$,
at $A_{2}^{\prime}$$$
\Delta+\delta=J+\left(n^{\prime}-n\right) e .
$$

It is worth noting that there is no need to apply the terms "ordinary" or "extraordinary" to the images $A_{1}^{\prime}$ and $A_{2}^{\prime}$ as, generally, in fullduplication instruments, all the rays were ordinary and extraordinary rays seriatim, or conversely (Figs. $3.20,3.25,3.27,3.28$ ). Throughout the following the left-hand image shall be denoted $A_{1}^{\prime}$. The interference theory shows that, when the path difference between the two interfering waves is not excessive, a typical tint is developed, featuring the abovementioned path difference. These tints are detected versus the path difference in Newton's scale shown hereunder. Therefore, three tints are perceived at $A_{1}^{\prime}, A_{2}^{\prime}$ and the remainder of the field. Let us assume that these tints correlate the path difference whose numerical values (in microns, for instance), deduced from Newton's scale are $a, b, c$, respectively. Then:
at $A_{1}^{\prime}$
$1-\left(n^{\prime}-n\right) e=a$,
at $A_{2}^{\prime}$$$
d+\left(n^{\prime}-n\right) e=b \text {. }
$$

Around $A_{1}^{\prime}$ and $A_{2}^{\prime}$

$$
\Delta=c
$$

whence

$$
\left(n^{\prime}-n\right) e=c-a=b-c=(b-a) / 2 .
$$


To preclude any errors in locating the tints, the following procedure is suitable: the polarizers are crossed and the microscope adjusted so that a dark field obtains $(\Delta=0)$, outside $A_{1}^{\prime}$ and $A_{2}^{\prime}$. The adjustment is continuously altered (leaving the polarizers alone) so as to show the colours sequentially until the field exhibits the purple of the first order $(\Lambda=c=0.565 \mu)$. Since the colours may be followed along Newton's scale of colours, no error need be expected in locating $c$. In this adjustment the colour is denoted by $a$ at $A_{1}^{\prime}$ and $b$ at $A_{2}^{\prime}$. Now the reverse procedure will enable one to locate these two colours. The microscope is adjusted, varying $\Delta$ so that the path difference $1-\left(n^{\prime}-n\right)$ is zero at $A_{1}^{\prime}$ which is now very dark. Referring once more to Newton's scale of colours while carrying out this operation will determine and, therefore, identify the initial colour at $A_{1}^{\prime}$, i.e. when $c=0.565 \mu$ on all sides of $A_{1}^{\prime}$ and $A_{2}^{\prime}$. Hence the colour surrounding $A_{1}^{\prime}$ and at $A_{1}^{\prime}$ are definitely determined:

(1) Purple (first order) on all sides of $A_{1}^{\prime}$,

(2) Colour correlated to the value $e$ at $A_{1}^{\prime}$.

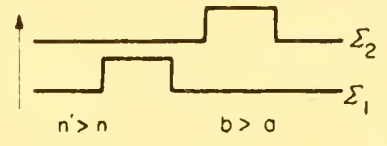

FIG. 7.4. First arrangement of the two wave surfaces.

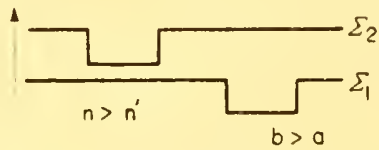

FIG. 7.5. Second arrangement of the two wave surfaces.

In fact, these two measurements are adequate since they evince $c$ and $a$, i.e. $\left(n^{\prime}-n\right) e$, according to the equation (7.4). There is no need, therefore, to determine the colour and, hence, the path difference at $A_{2}^{\prime}$. However, this may be done as a test. Having restored the first-order

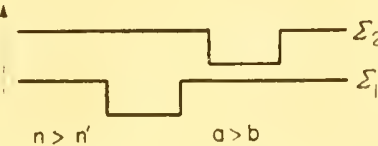

FIG. 7.6. Third arrangement of the two wave surfaces.

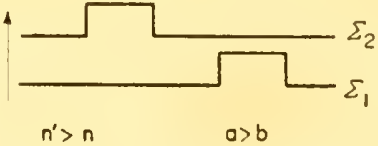

FIG. 7.7. Fourth arrangement of the two wave surfaces.

purple around $A_{1}^{\prime}$ and $A_{2}^{\prime}(c=0.565 \mu)$ the colour of the image $A_{2}^{\prime}$ is defined by $b$. Exactly the same operation is then resumed: 1 is varied so that black is developed in the image $A_{2}^{\prime}$. The colours being followed along Newton's scale of colours, it is possible to determine the initial 


\section{NEWTON'S COLOUR SCALE}

\begin{tabular}{|c|c|c|c|}
\hline & $\begin{array}{c}\text { Path } \\
\text { difference } \\
\text { in microns }\end{array}$ & $\begin{array}{l}\text { Interference colours between } \\
\text { crossed polarizers }\end{array}$ & $\begin{array}{c}\text { Interference colours between } \\
\text { parallel polarizers }\end{array}$ \\
\hline $\begin{array}{l}\text { First } \\
\text { order }\end{array}$ & $\begin{array}{l}0.000 \\
0.040 \\
0.097 \\
0.158 \\
0.218 \\
0.234 \\
0.259 \\
0.267 \\
0.275 \\
0.281 \\
0.306 \\
0.332 \\
0.430 \\
0.505 \\
0.536 \\
0.551\end{array}$ & $\begin{array}{l}\text { black } \\
\text { iron-grey } \\
\text { lavender-grey } \\
\text { greyish blue } \\
\text { clearer grey } \\
\text { greenish white } \\
\text { almost pure white } \\
\text { yellowish white } \\
\text { pale straw-yellow } \\
\text { straw-yellow } \\
\text { light yellow } \\
\text { bright yellow } \\
\text { brownish yellow } \\
\text { reddish orange } \\
\text { red } \\
\text { deep red }\end{array}$ & $\begin{array}{l}\text { bright white } \\
\text { white } \\
\text { yellowish white } \\
\text { brownish white } \\
\text { brownish yellow } \\
\text { brown } \\
\text { light red } \\
\text { carmine } \\
\text { dark reddish brown } \\
\text { deep violet } \\
\text { indigo } \\
\text { blue } \\
\text { greyish blue } \\
\text { bluish green } \\
\text { pale green } \\
\text { yellowish green }\end{array}$ \\
\hline $\begin{array}{l}\text { Second } \\
\text { order }\end{array}$ & $\begin{array}{l}0.565 \\
0.575 \\
0.589 \\
0.664 \\
0.728 \\
0.747 \\
0.826 \\
0.843 \\
0.866 \\
0.910 \\
0.948 \\
0.998 \\
1.101 \\
1.128 \\
1.151\end{array}$ & $\begin{array}{l}\text { purple } \\
\text { violet } \\
\text { indigo } \\
\text { sky-blue } \\
\text { greenish blue } \\
\text { green } \\
\text { lighter green } \\
\text { yellowish green } \\
\text { greenish yellow } \\
\text { pure yellow } \\
\text { orange } \\
\text { bright orange-red } \\
\text { dark violet-red } \\
\text { light bluish violet } \\
\text { indigo }\end{array}$ & $\begin{array}{l}\text { lighter green } \\
\text { greenish yellow } \\
\text { golden yellow } \\
\text { orange } \\
\text { brownish orange } \\
\text { light carmine } \\
\text { purplish red } \\
\text { violet-purple } \\
\text { violet } \\
\text { indigo } \\
\text { dark blue } \\
\text { greenish blue } \\
\text { green } \\
\text { yellowish green } \\
\text { impure yellow }\end{array}$ \\
\hline
\end{tabular}

tint $b$ in $A_{2}^{\prime}$. The measurements $b$ and $c$ evince $\left(n^{\prime}-n\right) e$ by means of equation (7.4). As stated previously, the arrangement achieved is not necessarily the one shown in Fig. 7.3. Figures 7.4, 7.5, 7.6 and 7.7 show the other contingent arrangements. There is no chance for errors as, when determining $a$ and $b$, if $b>a$. Fig. 7.4 must be considered when $n^{\prime}>n$ and Fig. 7.5 when $n>n^{\prime}$. When $b<a$, the figure obtaining is Fig. 7.6 when $n>n^{\prime}$, and Fig. 7.7 when $n^{\prime}>n$. 
The equations to be used are, therefore:

$$
\begin{aligned}
& b>a\left|\begin{array}{ll}
n^{\prime}>n & \left(n^{\prime}-n\right) e \\
n^{\prime}<n & \left(n-n^{\prime}\right) e
\end{array}\right|=c-a=b-c=(b-a) / 2 \\
& a>b\left|\begin{array}{ll}
n^{\prime}>n & \left(n^{\prime}-n\right) e \\
n^{\prime}<n & \left(n-n^{\prime}\right) e
\end{array}\right|=a-c=c-b=(a-b) / 2
\end{aligned}
$$

These data are not altered if the two waves $\Sigma_{1}$ and $\Sigma_{2}$ are intersected as shown in Fig. 7.3, i.e. if $\delta>1$. There remains to determine whether $n^{\prime}>n$ or $n^{\prime}<n$. To solve the problem once and for all, a sample (whose index is known), is measured.

Let us take a numerical example:

(1) Testing the adjustment. A sample, whose index is known to be $n=1.550$ and of thickness $e=10 \mu$, is immersed in a medium (index $\left.n^{\prime}=1 \cdot 527\right)$. Then, $\left(n-n^{\prime}\right) e=0.23 \mu$.

Having set the instrument for the first-order purple to be developed around both images, these appear in two different shades. $A_{1}^{\prime}$ is, as before, still the left-hand image and $A_{2}^{\prime}$ the right-hand one. The tints observed in both images are correlated to the path differences $0.565+$ $+0.23=0.588 \mu$ and $0.565-0.23=0.542 \mu$. According to Newton's scale, the image correlated to $0.588 \mu$ is indigo and the one correlated to $0.542 \mu$ is red. If it is found that the left-hand image $\left(A_{1}^{\prime}\right)$ is indigo, $a=0.588 \mu$ and the arrangement is either that shown in Fig. 7.6 or 7.7. In the present case, the sample is determined $\left(n>n^{\prime}\right)$, and the figure observed is correlated to Fig. 7.7. In this way the position of the microscope element which can take effect on $\Delta$ can be determined. In this position, the left-hand image $\left(A_{1}^{\prime}\right)$ forms part of the lower wave (the direction of light is shown next to each figure). If, now, an undetermined sample is dealt with, it is ascertained that, for above mentioned position of the adjusting element, the only possible arrangements are those shown in Figs. 7.4 and 7.6.

(2) Measuring the path difference of an undetermined sample. The sample is immersed in a medium, of known index $n^{\prime}$. Observation in the polarizing interference microscope discloses that when the field around the images is purple $(c=0.565 \mu)$, the left-hand image $\left(A_{1}^{\prime}\right)$ is bright yellow $(a=0.332 \mu)$ and the right-hand image $\left(A_{2}^{\prime}\right)$ green ( $b$ from $0.747 \mu$ to $0.826 \mu$ ). Then, $b>a$. However, calibrating the known sample reveals that the left-hand image belongs to the lower wave, thus correlating with Fig. 7.4. The sample index $n$ is lower than the index of the medium in which the sample is immersed. Detecting 
the colour of the image $A_{1}^{\prime}$ then gives:

$$
n<n^{\prime} \quad\left(n^{\prime}-n\right) e=0.565-0.332=0.233 \mu .
$$

Note 1. The first-order purple was selected as field colour surrounding the images $A_{1}^{\prime}$ and $A_{2}^{\prime}$ merely because it is readily detectable. Naturally, all measurements can be resumed selecting another background colour and testing the data as often as required. Thus, in the previous numerical example, if the microscope is adjusted for $c=0.332 \mu$ the field surrounding the images is bright yellow instead of purple. The left-hand image $\left(A_{1}^{\prime}\right)$ becomes lavender grey $(a=0.097 \mu)$ but the difference $0.332-0.097=0.235 \mu$ remains the same.

Note 2. The flat-tints process just described does not apply when dispersions of the medium $n$ (the object) and the medium $n^{\prime}$ (medium encompassing the object) differ overmuch as tints depend on the variations of the ratio $\left(n-n^{\prime}\right) e / \lambda$ in the visible spectrum. When changing over from the radiation $F(0.480 \mu)$ to the radiation $C(0.565 \mu)$ the previous ratio takes on the values $\left(n_{F}-n_{F}^{\prime}\right) e / \lambda$ and $\left(n_{C}-n_{C}^{\prime}\right) e / \lambda$. Therefore, it is the difference between $\left(n_{F}-n_{F}^{\prime}\right)$ and $\left(n_{C}-n_{C}^{\prime}\right)$ that takes effect. Such difference, equating $\left(n_{F}-n_{C}\right)-\left(n_{F}^{\prime}-n_{C}^{\prime}\right)$ is the difference between dispersions. If the tints are to remain the same as in Newton's scale, the numerator of the ratio $\left(n-n^{\prime}\right) e / \lambda$ must not be a function of $i$. Consequently, $n-n^{\prime}$ must remain constant, i.e. dispersions must be the same. Otherwise the tints are no longer those in Newton's scale and cannot be detected.

\section{MEASURING PATH DIFFERENCES, APPLYING THE FRINGES PROCESS (FULL-DUPLICATION PROCESS)}

Measurements may be carried out by substituting tint discrimination for interference-fringe shifts. Such fringes can be originated in several ways, but, regardless of the device employed, phenomena remain the same. The following example should make it easier to comprehend origination and application of these fringes. Let us revert to the diagrammatic microscope shown in Fig. 3.19 and work in monochromatic light. We have seen (Chapter III) that a slit, located in the condenser focus (not shown in Fig. 3.19), should be used: it is imaged in the focus of the objective $O_{1}$, where the Wollaston prism $W$ lies. The imaged slit is to be aligned with one of the Wollaston-located fringes. Let us shift slightly the Wollaston $W$ in the direction of the microscope axis by moving it, for instance, away from the objective. 
Since the slit is no longer imaged on the Wollaston fringes, this image is the more duplicated as the Wollaston is moved farther away from the focal plane of the objective $O_{1}$. Figure 7.8 shows the originated beams from the slit-images $F^{\prime}$ and $F^{\prime \prime}$ given by the Wollaston. Phe-

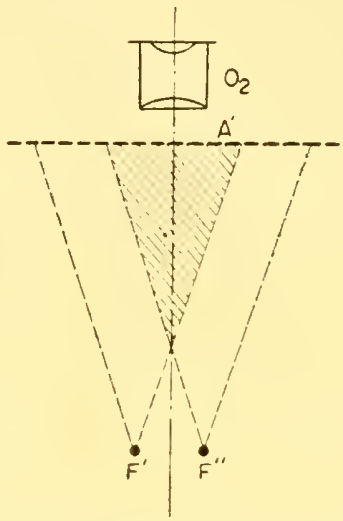

FIG. 7.8. Fringes produced by the slit-images $F^{\prime}$ and $F^{\prime \prime}$.

nomena occur as if the image-plane $A^{\prime}$ were illuminated by the two synchronous sources $F^{\prime}$ and $F^{\prime \prime}$. Therefore, veritable Young's fringes are observed in said image plane.

Since changing over from one fringe to the next causes the path

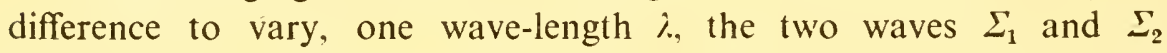
are arranged in the image $A^{\prime}$ as shown in Fig. 7.9. The fringes outline

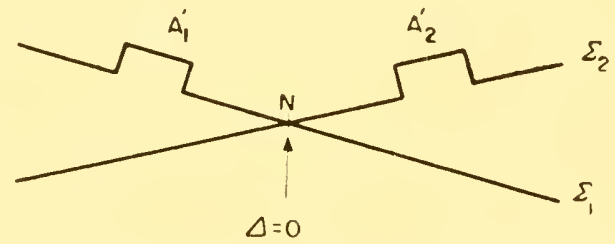

FIG. 7.9. The 1wo wave surfaces when fringes are developed.

equal path-difference loci, i.e. loci which are as far apart as the waves $\Sigma_{1}$ and $\Sigma_{2}$. The fringes involved are, therefore, straight equidistant and parallel lines at right angles to the plane of Fig. 7.9. In white light and with crossed polarizers. the dark fringe $(\Delta=0)$ would be developed at $N$. The path difference increases on both sides and colour fringes are perceived. In monochromatic light, the field is streaked with bright and dark fringes. In Fig. 7.10 and following, 
in order to simplify the figures, the dark fringes are shown as dark lines, narrower than the bright inter-spaces (bright fringes), sandwiched between them. It is assumed that settings are made on the dark fringes in order to make the figures clearer. Figure 7.9 shows why the fringes are at right angles to the direction of duplication. The field has the appearance shown in Fig. 7.10. The area $B C$ in Fig. 7.10, etc. are correlated to the area $B C$ in Figs. 6.1 and 6.2.

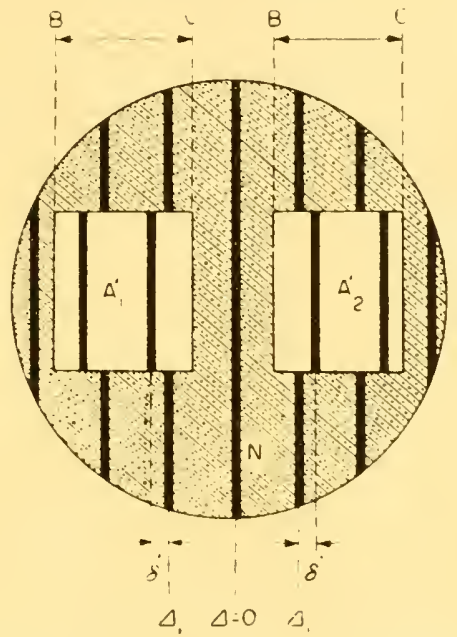

FIG. 7.10. Field appearance when the fringes are at right angles to the direction of duplication.

In the areas $A_{1}^{\prime}$ and $A_{2}^{\prime}$, the fringes are not in alignment with the other fringes since the path difference is not the same. In one of the images ( $A_{1}^{\prime}$, for instance) the shift, away from the outer fringes (area where there is no object, i.e. (1), (2), (3) in Fig. 7.1), equates, but is of opposite sign to the shift in the other image $\left(A_{2}^{\prime}\right)$, if the fringe $N(\Delta=0)$ is half-way between $A_{1}^{\prime}$ and $A_{2}^{\prime}$. Phenomena are symmetrical in relation to $N(\Delta=0)$. Let us detect the next fringe (outside either $A_{1}^{\prime}$ or $A_{2}^{\prime}$ ). Such a fringe corresponds to a certain path difference $\Delta_{1}$ (Fig. 7.10) and, then, $d_{1}=\lambda$. This means that, changing over from the fringe $N$ (corresponding to a zero path difference) to the fringe $\Delta_{1}$, the path difference changes over from zero to $\Delta_{1}$. The object originates a shift $\delta^{\prime}$ which amounts to a certain fraction $K^{\prime}$ of a fringe inter-space. Each such space equates a path difference amounting to one wavelength $\lambda$ of the light involved. Then, in the present instance:

$$
\delta^{\prime}=K^{\prime} \lambda \text {. }
$$


As shown in Fig. 7.10, the shift amounts approximately to one third of the space between two fringes. Then, $K^{\prime}=1 / 3$. However, the shift $\delta^{\prime}$ does not necessarily equate the actual path-difference $\delta$ brought in the object as, in monochromatic light, all fringes are identical. Now, were the object-originated path difference to equate a wavelength whole number $p$, nothing would be visible in monochromatic light. Were a shift $\delta^{\prime}=K^{\prime} \lambda$, smaller than a fringe inter-space, to be observed, then, the actual shift may equate a whole number times $i$, viz., $p \lambda$, plus the fraction $K^{\prime}$. The actual path-difference $\delta$ would be:

$$
\delta=\left(n^{\prime}-n\right) e=p \hat{\imath}+\delta^{\prime}=p \hat{\lambda}+K^{\prime} \lambda=K \lambda .
$$

In Fig. 7.10, the path-difference $\delta^{\prime}$ is the actual path-difference if. in the case of the image $A_{1}^{\prime}, p$ is zero and the shift is to be located in relation to the right-hand fringe. How to eliminate these difficulties is shown later.

In the above-described method, the fringes are at right angles to the direction of duplication. Such a direction is not too con-

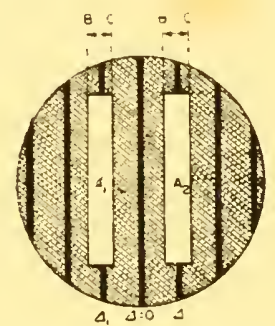

FIG. 7.11. Lengthwise extended object at right angles to the direction of duplication.

venient for carrying out measurements when the object extends over some distance in a given direction or when the straight edge of a phase-shifting object is being investigated. Such a lengthwise extended object appears as shown in Fig. 7.11: owing to the shift. it

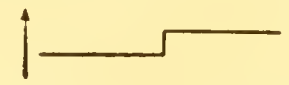

FIG. 7.12. Transmitted wave by a straight edge of a dephasing object.

may occur that no dark fringe develops in the two images. The straight edge of a phase-shifting object gives rise to a wave similar to the one shown in Fig. 7.12 or looks like the Fig. 7.13: devoid of 
visible fringe in the duplicated area. Of course, in the overlapped area $a b c d$, the fringes are in alignment with the outer fringes. Then, measurements can be carried out by setting the extended edge of the object at $45^{\circ}$ from the fringes (Figs. 7.14 and 7.15). Duplication is less marked but the fringe shift $\delta^{\prime}$ is readily observed.

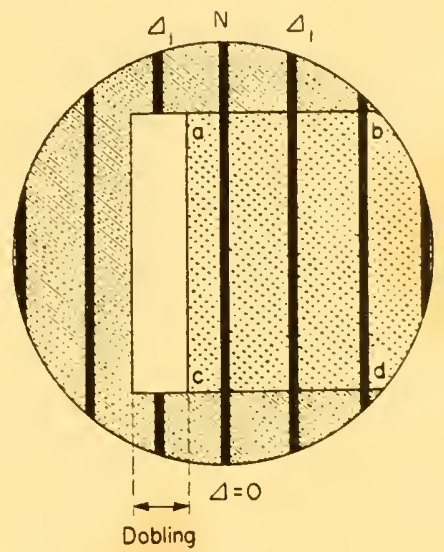

FIG. 7.13. Straight edge of a dephasing object at right angles to the direction of duplication.

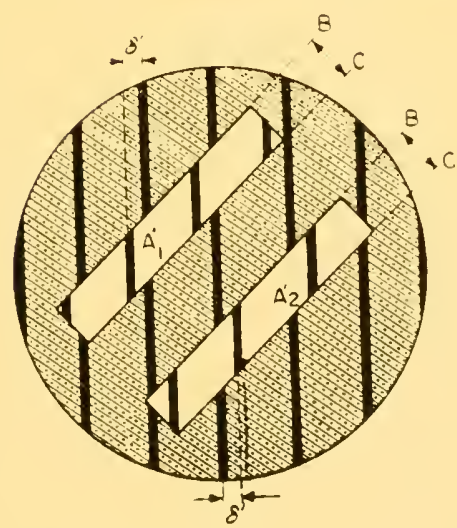

FiG. 7.14. Measurements when the extended edge of an object is at $45^{\circ}$ from the fringes.

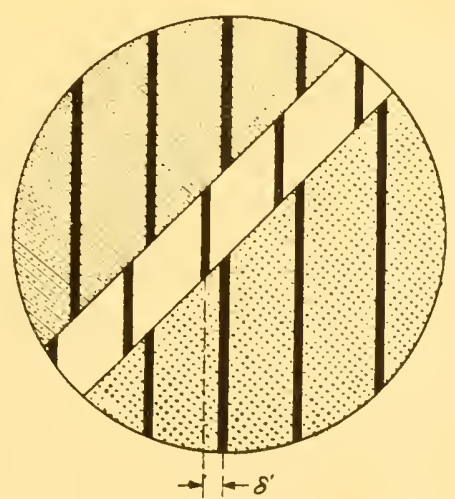

FIG. 7.15. Measurements when the straight edge of an object is at $45^{\circ}$ from the fringes.

Nevertheless, it is advisable, when these two types of objects are involved, to have the fringes at right angles to the direction corresponding to the larger dimension. It might occur that merely rotating the object $90^{\circ}$ from the positions shown in Figs. 7.11 and 7.13 would be 
adequate. Such is not the case for the extended object shown in Fig. 7.11 for both images are overlapped at abcd and the shift hardly observable in the two areas $a a_{1} c c_{1}$ and $b b_{2} d d_{2}$ (Fig. 7.16). As regards the straight edge of a phase-shifting object it is unobservable (Fig. 7.17) since duplication is invisible as it parallels the straight edge of the object.

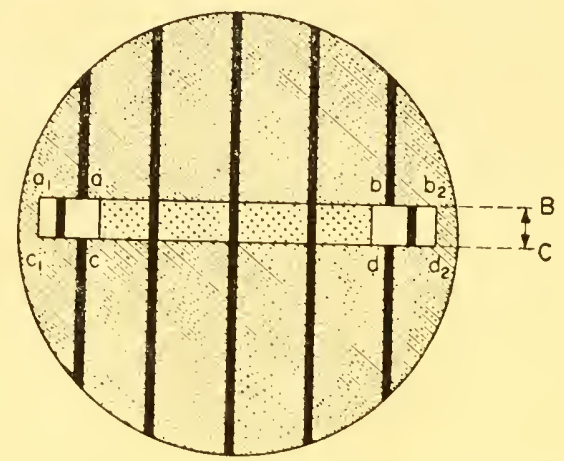

FIG. 7.16. Extended object parallel to the duplication.

Therefore, fringes must be paralleled to the duplication and the object's larger dimension be perpendicular to the fringes. This is the most convenient and reliable arrangement when precise measurements are required.

In polarizing interference microscopes, the fringes are usually paralleled to duplication and all the operator has to do is to set the

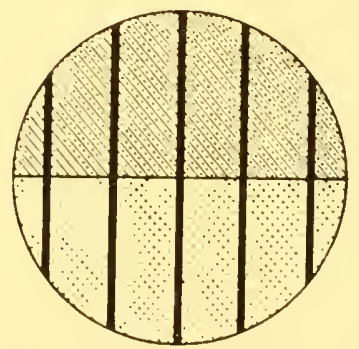

FIG. 7.17. Straight edge of an object parallel to the duplication.

object in the proper direction this being readily achieved with a little practice.

There are several methods for paralleling fringes to duplication. Several of these are shown later. For the time being, this adjustment. 
is assumed to have been carried out and when a strip-shaped object is involved the result is shown in Fig. 7.18.

As previously, $\delta$ is reckoned in fringe inter-spaces: if $\delta$ equates $p$ inter-spaces, plus or minus the fraction $K^{\prime}$, the equation (7.8) provides the path-difference $\delta$. Now, the following method should be used to avoid the uncertainty regarding the actual path-difference, i.e. the value of $K$ in the equation (7.9) provided, however, that the dispersions of both object and medium are not too different. Let us substitute monochromatic light for white light.

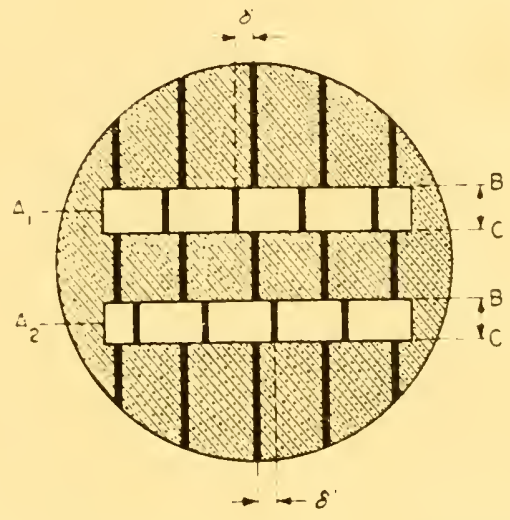

FIG. 7.18. Fringes parallel to the duplication.

The dark fringe outside the two images $A_{1}^{\prime}$ and $A_{2}^{\prime}$ and within either of the two latter are duly located (Fig. 7.19) by means of the reticle of the eyepiece micrometer. Then, observation is made in monochromatic light of wave-length $\%$ (Fig. 7.20). In the case of Figs. 7.19 and 7.20 , it is evinced at once that $p=3$ and $K^{\prime}=1 / 3$. if $K^{\prime}$ is computed in relation to the right-hand fringe. In the example selected, the path-difference is, therefore, $\delta=10 \lambda / 3$. Merely observing the direction of the fringe shift, using a sample of predetermined index, determining whether $n>n^{\prime}$ or $n^{\prime}>n$. For instance, it may be found that, in the upper image $\left(A_{1}^{\prime}\right)$, the fringe shift is taking place to the right if the sample index is higher than that of the immersion medium. An object causing the fringes to shift to the left in relation to the outer fringes is then correlated to a lower index. The direction of shift is observed in white ligth. There is no problem when the object tapers towards its edges and no white light change-over is then required. All that matters is to follow up a specific fringe (cf. Chapter VI, § 1). 
In industrial instruments, actuating a microscope element usually gives rise to parallel-to-duplication fringes. Yet it may be desirable to be aware of simple processes providing such fringes. Here are two of them. Both are of general application but, in order to simplify matters, the microscope discussed is the one shown in either Fig. 3.19 or 3.20. It is assumed that the Wollaston prism $W_{2}$ is in its normal position, i.e. in the focus of the objective $O_{1}$, as in the flat-tints method.

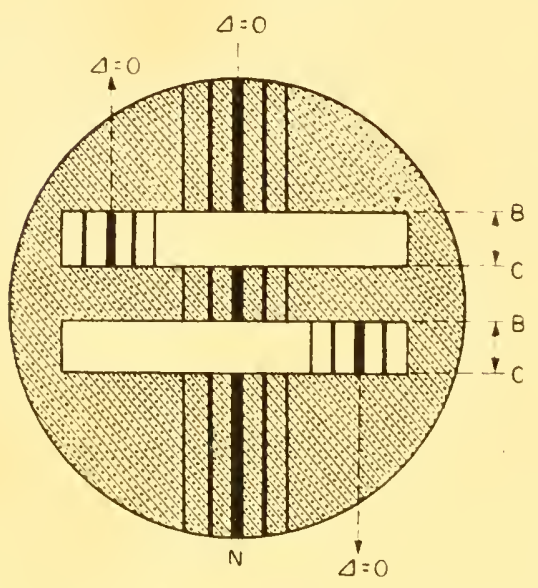

FIG. 7.19. Setting of the black fringe within and outside the two images.

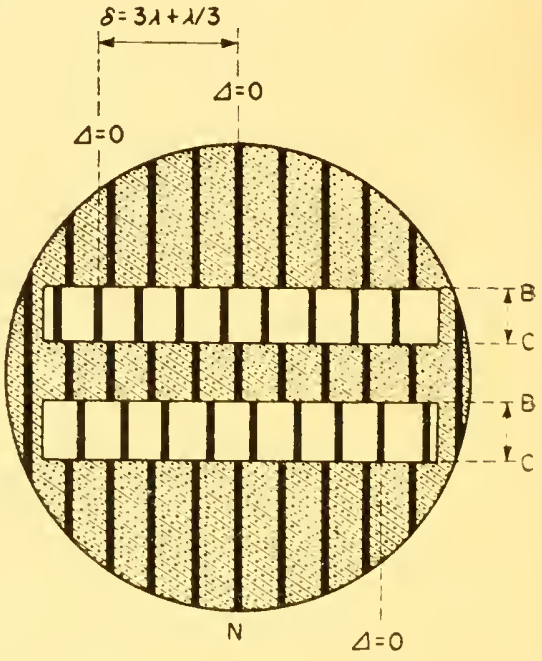

FIG. 7.20. Actual path difference measurement in monochromatic light.

Let us set a third prism, $W_{3}$, in the image plane $A^{\prime}$ (Fig. 7.21), the prisms $W_{1}, W_{2}, W_{3}$ being, of course, between the polarizers $P_{1}$ and $P_{2}$. The image $A^{\prime}$ and the $W_{3}$ fringes are concomitantly perfectly sharp. If there are any path differences in the object, i.e. in the image $A^{\prime}$. the $W_{3}$ fringes are shifted. It should be noted that $W_{3}$ does not give rise to any duplication in the image since the focalizing plane of its fringes are merged into the image plane.

Instead of the $W_{3}$ prism, a Savart polariscope (or the polariscope shown in Fig. 4.29(a)) may be set above the eyepiece (Fig. 7.22). As always, both $W_{1}, W_{2}$ and the polariscope $S$ are to be between the polarizers $\mathcal{P}_{1}$ and $\mathcal{P}_{2}$. A convergent light beam passes through the Savart $S$, set next after the eyepiece, originates at infinity fringes which are virtually straight, parallel and equidistant. Properly adjusted, the microscope shows the ultimate image, that of $A^{\prime}$, to be at infinity. 
Therefore, the ultimate image of the object and the Savart fringes are seen concurrently. Any change in the optical path in object, i.e. within the image, shifts the fringes of the Savart $S$.

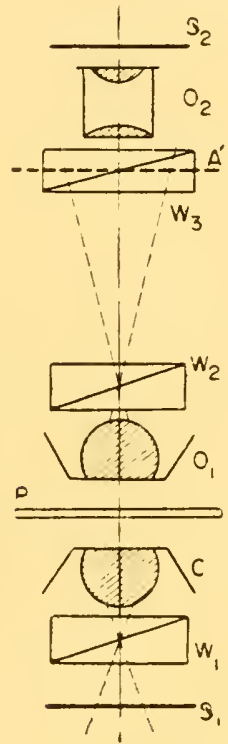

FIG. 7.21. Fringes parallel to the duplication (Wollaston).

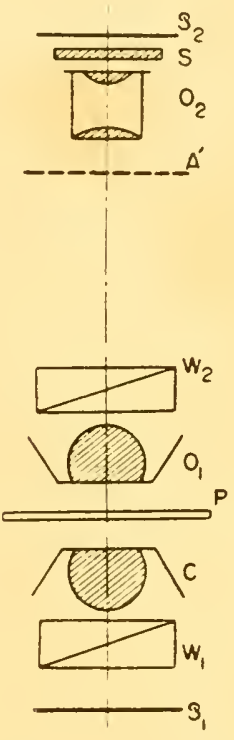

FIG. 7.22. Fringes parallel to the duplication (Savart).

\section{DETERMINING THE REFRACTION INDEX FROM PATH-DIFFERENCE MEASUREMENT (FULL-DUPLICATION PROCESS)}

As in all interference methods, the above processes evince an optical path, i.e. the product of an index by a thickness. If $n$ is to be measured. $e$ must be determined and vice versa. Let us assume $e$ to be known.

\section{Flat-tints method}

When $b>a$ and $n>n^{\prime}$, the equation (7.5) yields:

$$
n=n^{\prime}+(c-a) / e \text {. }
$$

Other instances are easily derived from the equations (7.5).

Since $e$ and the index $n^{\prime}$ of the medium encompassing the object, are known, merely measuring $c$ and $a$ enables one to educe the index $n$ of the object. 
Let us take the numerical instance mentioned in $\$ 1$ : If $e=20 \mu$ and $n^{\prime}=1.525$ the equation (7.6) yields:

$$
n=n^{\prime}-\frac{0 \cdot 233}{20}=1 \cdot 52 \text {. }
$$

As stated above, the index is only approximated. Accuracy does not extend beyond two places of decimals.

Fringe-shifting method

When $n<n^{\prime}$, the equation (7.8) yields:

$$
n=n^{\prime}-K \lambda / e \text {. }
$$

As $e$ and $n^{\prime}$ are determined. measuring the shift $K$ of the figures now evinces the index $n$ of the object. Reverting to the previous example ( $n^{\prime}=1.527$ and $e=20 \mu$ ) it is found that the fringe shift of the spectral green line of mercury $(\lambda=0.546 \mu)$ equates the fraction $K=1 / 2 \cdot 27$ of the fringe inter-space. Then:

$$
n=1 \cdot 527-\frac{0 \cdot 546}{45 \cdot 4}=1 \cdot 515
$$

Accuracy depends on the precision with which $e$ and $K$ are known. Assuming that $e$ be measured with a one-micron accuracy: de/e $=1 / 20$. If $K$ is determined with an accuracy within 1/40 of the fringe inter-space, then, $|K| K=1 / 20$ since $K \simeq 1 / 2$. The relative error $\mathcal{I}\left(n^{\prime}-n\right) /\left(n^{\prime}-n\right)$ is derived from:

$$
\frac{\Delta\left(n^{\prime}-n\right)}{n^{\prime}-n}=\frac{d e}{e}+\frac{1 K}{K} \simeq 0 \cdot 1
$$

whence

$$
I\left(n^{\prime}-n\right) \simeq 0.001
$$

In the example selected, the accuracy attained is correct to one unit of three decimals. As stated previously, the aperture of the incident beam is a prominent factor in measurement accuracy (cf. Chapter VIII, \$2).

When the thickness $e$ is not known

The Barer process consists in immersing the object seriatim, when feasible, in two media whose indices $n_{1}^{\prime}$ and $n_{2}^{\prime}$, are known. Now. 
using the fringe-shift method, the shifts $K_{1}$ and $K_{2}$ are detected, respectively, in the media $n_{1}^{\prime}$ and $n_{2}^{\prime}$. Whence the two equations:

$$
n=n_{1}^{\prime}-\frac{K_{1} \lambda}{e} \quad n=n_{2}^{\prime}-\frac{K_{2} \lambda_{2}}{2}
$$

from which are derived both index and thickness:

$$
n=\frac{n_{2}^{\prime} K_{1}-n_{1}^{\prime} K_{2}}{K_{1}-K_{2}} \quad e=\frac{\left(K_{1}-K_{2}\right) \hat{\lambda}}{n_{1}^{\prime}-n_{2}^{\prime}} .
$$

\section{MEASURING THE REFRACTION INDEX OF LIQUIDS (FULL-DUPLICATION PROCESS)}

Köfler propounded a method based on employing glass powders whose indices are known (cf. Chapter XI. $\S 5$ ). A specific powder is immersed in the liquid to be investigated. Observations are carried out on a stage provided with an adjustable heating device. The microscope objective has a low aperture and the condenser is diaphragmed to provide coherent light. Shifting slightly the microscope settings evinces a luminous fringe, called the "Beck line", which surrounds every glass particle. As the microscope is moved away from the specimen, the Beck line is moving towards the higher refracting index medium. Conversely, as the microscope nears the specimen, it moves towards the lower refracting index medium. Observing the Beck line enables one to determine which way the temperature is to be adjusted for the glass particles to vanish, i.e. to obtain equal indices. At the temperature considered and the wave-length used, the liquid has the same index as the glass particles.

Interference processes enable measurement of the refraction index without any temperature adjustment. The procedure is to measure the specimen at its own temperature. Were the temperature to vary, the relevant refraction-index changes are followed up. E. E. Jelley has made use of mulit-wave interferences. The more readily applied polarizing interference methods will now be discussed.

\section{Using a micro-chamber}

The 2 cover-glass plates, $d$ and $d^{\prime}$, whose index $n^{\prime}$ is known, are secured to the object-slide $a$ (Fig. 7.23). The liquid (whose index $n$ is to be measured) is poured in a minute space provided between the two plates. This arrangement is covered by the object-slide $b$. The two plates, $d$ and $d^{\prime}$, need not touch either $a$ or $b$ and only the thick- 
ness $e$ of the plates take effect. There may be some liquid between $a$ or $b$ and the plates $d$ and $d^{\prime}$ without this interfering with measurements. The arrangement is set on the stage of a polarizing interference microscope. The liquid area has the shape of a rectangle which is set lengthwise at right angles to the fringes, whereupon the procedure mentioned in $\$ 3$ (fringe-shift method) is applied. Provided $n^{\prime}>n$, a flat wave. after passing through the micro-chamber, has the shape shown in Fig. 6.2. The area $B C$ in this figure corresponds to the

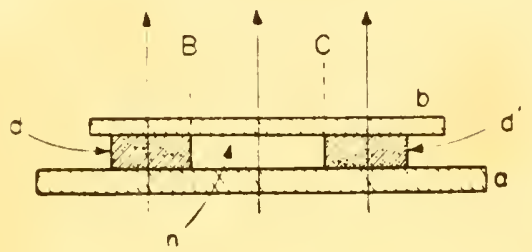

FIG. 7.23. Micro-chamber for index measurements (liquids).

area $B C$ (index $n$ ) in Fig. 7.23. The flat areas on either side of $B C$ (Fig. 6.2) correspond to the surface-wave areas that travelled through the plates $d$ and $d^{\prime}$ of index $n^{\prime}$ (Fig. 7.23). The aspect of the field is shown in Fig. 7.18. The two images $A_{1}^{\prime}$ and $A_{2}^{\prime}$ are images of the area $B C$ (Fig. 7.23) where is the liquid $n$. Let $K$ be the fringe shift expressed as fringe interspace, then (equation 7.9):

$$
\left(n^{\prime}>n\right) \quad n=n^{\prime}-K i_{1} / e .
$$

Let us consider $n^{\prime}-n=0.02, e=0.17 \mathrm{~mm}$ and $J e=2 \mu$. Measuring $K$ with an accuracy of approximately $1 / 40$ of the space between two fringes $(\lambda=0.546 \mu), n$ is correct within 2 to 3 units of four places of decimals. Owing to temperature-originated index variations of the liquid, the work is to be carried out at a steady temperature. As mentioned in $\$ 2$, to preclude any error on $K$, a change-over to white light is required. Therefore. dispersion of the liquid and the plates $d$ and $d^{\prime}$ should be not too different. $d$ and $d^{\prime}$ may be substituted (Fig. 7.23) for two plates whose faces $B$ and $C$ are sloping and not perpendicular to the object-slide. Under such conditions, fringe deformation can be followed up in monochromatic light thus dispensing with white light (Chapter VI. $\$ 1$ ). Using a liquid whose index is known, the fringe-shift direction is noted. Merely comparing this shift with the one obtained from the undetermined liquid $n$ shows whether $n$ is higher or lower than $n^{\prime}$. 


\section{APPLICATION OF THE DIFFERENTIAL METHOD}

Polarizing interference microscopes, based on the differential method, are suitable for measuring refraction indices of transparent isotropic objects. These instruments are not so commonly used as the full-duplication types, but they should be used whenever feasible owing to their simple construction. As in the full-duplication method, measurements are based on a prior determination of the path difference. Either the flat-tints or the fringe-shift method is applicable to such deierminations. All the following measurements can be made with the microscopes shown in Figs. 3.25, and 3.28. Provided that the duplication brought about by $W_{2}$ in the image $P^{\prime}$ be feeble, the microscope, shown in Fig. 3.20, is also suitable.

\section{MEASURING PATH DIFFERENCE USING THE FLAT-TINTS METHOD (DIFFERENTIAL PROCESS)}

As previously, and in order not to over-elaborate the present work, we will consider a diagrammatic transparent object (Fig. 7.24). In the areas $b_{2}$ and $b_{1}$, i.e. to the left of $B B^{\prime}$ and the right of $C C^{\prime}$, there is the same medium of index $n^{\prime}$, and another medium of index $n$ in the area between $B B^{\prime}$ and $C C^{\prime}$. Such media are separated by flat surfaces

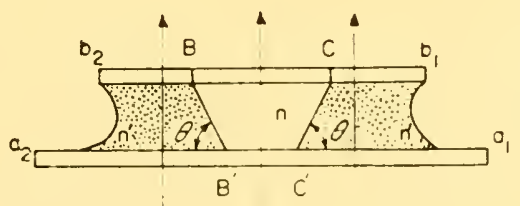

FIG. 7.24. Diagrammatic transparent object $B C B^{\prime} C^{\prime}$.

perpendicular to the plane of Fig. 7.24 which are projected along $B B^{\prime}$ and $C C^{\prime}$. Their angle in relation to the object-slide is $\theta$. The object, whose index is being measured, consists of the $n$ index medium comprised between $B B^{\prime}$ and $C C^{\prime}$. The microscope is illuminated in white light. Let us assume $n<n^{\prime}$. Prior to travelling through the specimen, the wave-surface is the plane surface-wave $\Sigma_{0}$ (Fig. 7.25). After travelling it is deformed and becomes the wave-surface $\Sigma_{1}$. The areas $B b_{2}$ and $C b_{1}$ are lagging in relation to the area $B^{\prime} C^{\prime}\left(n<n^{\prime}\right)$. Owing to the birefringent system in the interference microscope, the wave $\Sigma$ is duplicated. The two waves, $\Sigma_{1}$ and $\Sigma_{2}$, are in the image plane 
(Fig. 7.26). Their side shift is small in relation to the width of the sloping areas $B B^{\prime}$ and $C C^{\prime}$. The magnitude of $d$ is so selected as to be close to the microscope's resolving power in order that the differential method be applicable to all objects without any objectionable

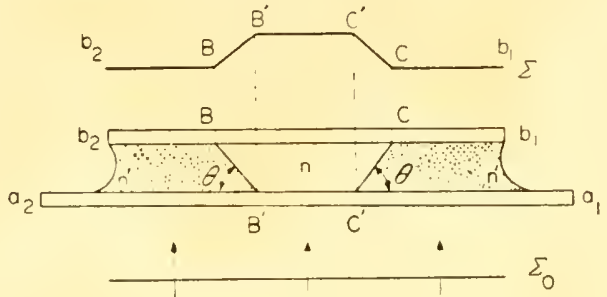

FIG. 7.25. After passing through the object, the wave surface is $\Sigma$.

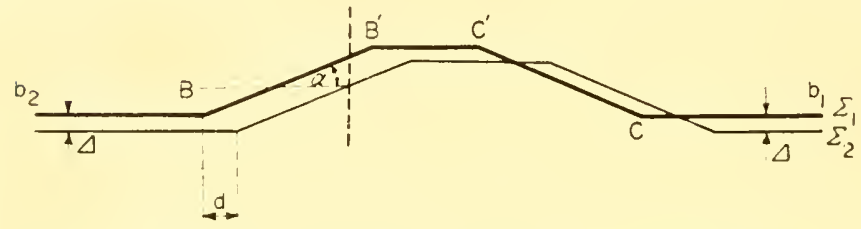

FJG. 7.26. The two waves $\Sigma_{1}$ and $\Sigma_{2}$ in the differential process.

duplication which, thus, remains invisible. To make Fig. 7.26 clear, the shift $d$ and the slope of the surface waves $\Sigma_{1}$ and $\Sigma_{2}$, are considerably amplified. The angle $\theta$ (Fig. 7.25) is not necessarily small but $i t$ is assumed that the angle $a$ does remain so ( $n$ being closer $n^{\prime}$ as $\theta$ is larger). Let $\perp$ be the path difference between the two waves

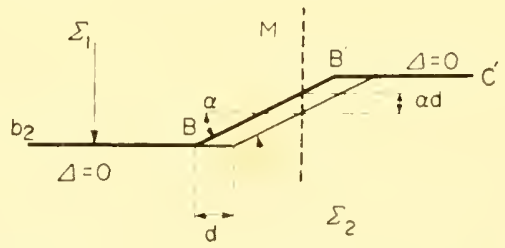

FiG. 7.27. The two waves $\nu_{1}$ and $\nu_{2}$ when $1=0$.

$\Sigma_{1}$ and $\Sigma_{2}$ in the areas where they are plane and parallel (areas $B b$. and $\left.C b_{1}\right)$. Actuating the proper microscope control will adjust at will the path difference 1 . Owing to the slope a of the wave-surfaces, the path difference, in the area $M$, is not the same as $\angle$. In Fig. 7.27. only the left part of Fig. 7.26 is shown and $1=0$. Figure 7.27 shows 
readily that the path difference between $\Sigma_{1}$ and $\Sigma_{2}$, equates at $M$ the product $a d$. If the path difference $I$ is not zero, Fig. 7.28 shows that the path difference at $M$ becomes $\Delta+a d$. When $\Delta=0.565 \mu$, the first order purple is oblained between crossed polarizers, in the areas $B b$. and $B^{\prime} C^{\prime}$. Since the path difference at $M$ has not the same value,

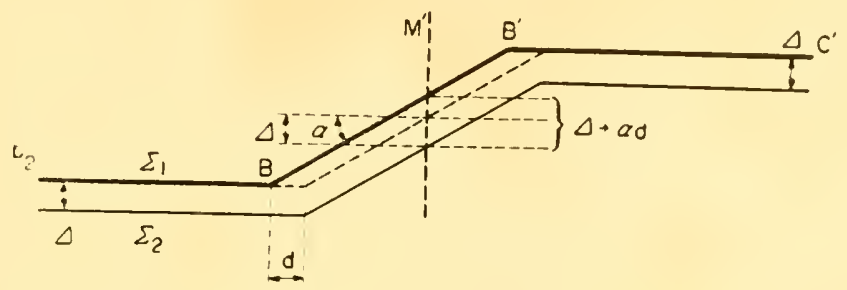

FIG. 7.28. Owing to the slope $\alpha$ the path difference at $M$ is $1+a d$.

the tint is different and characierizes the slope $\alpha$ of the wave-surface as the side shift $d$ is a constant of interference microscope. The tints in the areas $B b_{2}$ and $M$ are identified by means of Newton's scale. Let $a$ and $b$ be the correlated path differences, i.e. in microns. Then:

$$
\begin{aligned}
1 & =a \\
1+a d & =b
\end{aligned}
$$

whence the slope $\alpha$ :

$$
a=(b-a) / d .
$$

As shown later, connecting $\alpha$ to the index $n$ is readily achieved. The shift $d$ is set in a definite direciion in relation to the microscope. To make equations (7.12) and (7.13) applicable, the steeper line of the object, i.e. $B B^{\prime}$ in the present instance, must be parallel to the direction of duplication. To do this, after securing the firstorder purple, in the area $B_{2}$, the specimen is rotated (by means of the siage) until the tint in the area $B b_{2}$ is farthest from the purple in Newton's scale. If this adjustment is not achieved, the duplication in equations (7.12) and (7.13) is not $d$ but $d \cos \beta$ where $\beta$ is the angle formed by the direction of actual duplication and projection of the steeper slope $B B^{\prime}$ to the horizontal plane (stage plane). If the projected steeper-slope line $B B$ to the horizontal plane is at right angles to the direction of duplication, then: $\beta=\pi / 2$ and $d \cos \beta=0$. The object's slope is invisible since the path difference is the same at $B b_{2}$ and $B B^{\prime}$ ( $a=b$ in equation (7.12)). 
In Fig. 7.28, the path difference 1 and $a d$ add up. In Fig. 7.29, they are substracted from one another and $a d-1$ should be written in equation (7.12). This is not very important but it should be determined whether the wave surface shows a peak (Fig. 7.30) or a irough (Fig. 7.31). Merely substituting the specimen for an object whose slope and index are known provides the answer. For instance, let us consider an object

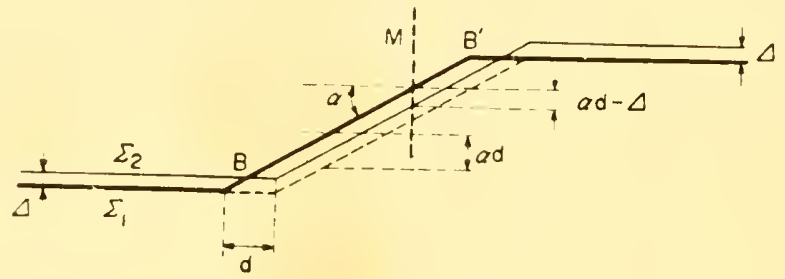

FIG. 7.29. The path difference at $M$ is $a d-1$.

with the slope $\theta=20^{\circ}$. Let us assume $n=1.515$ and $n^{\prime}=1.526$ and that the duplication brought about by the interference microscope in the specimen plane $d=25 \mu$. The microscope is adjusted so that $A=0$, i.e. $B b_{2}$ is dark. At $B B^{\prime}$, the tint is, for instance, lavender grey $(0.097 \mu)$. Both waves are arranged as shown at $B B^{\prime}$. Now the adjustment is altered so as to increase 1 : the tint becomes white at $B B^{\prime}$. This means that the path difference increases there. Hence, it is the wave most shifted towards the right by duplication

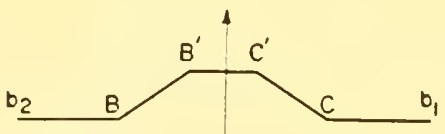

FIG. 7.30. Wave surface when $n^{\prime}=n$.

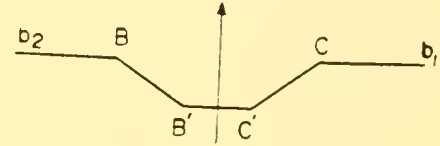

Fig. 7.31. Wave surface when $n^{\prime}<n$.

(wave $\Sigma_{2}$ ) that moves away from $\Sigma_{1}$ as it drops. Or, which amounts to the same thing, it is the wave most shifted to the left by duplication $\left(\Sigma_{1}\right)$ that moves away from $\Sigma_{2}$ as it rises. Therefore, observing an object of known index $n$ shows that as 1 is increased from zero, the tint order at $B B^{\prime}$ rises if $n<n^{\prime}$.

Let us now assume that the index $n$ of the known object is higher than $n^{\prime}$. Starting again from the value $l=0$, the arrangement is shown in Fig. 7.32. Lei us increase 1 by aciuating the microscope control as mentioned before. The wave $\left(\Sigma_{2}\right)$ most shifted to the right drops, or, which amounts to the same thing, the wave most shifted 
to the left $\left(\Sigma_{1}\right)$, rises. In the area $B B^{\prime}$, the path difference decreases. If the initial tint $(\triangle$ being $=0$ ) is still lavender grey, it now tends to a darker tone.

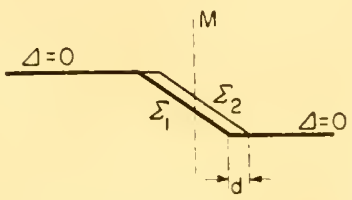

FIG. 7.32. The two wave surfaces when $n>n^{\prime}$.

In this way, the path difference at $B b_{2}$ ( $a$ in equation 7.12) and at $B B^{\prime}$ ( $b$ in equation 7.12) are measured unquestionably and it is also ascertained that the surface wave $\left(\Sigma_{1}\right.$ or $\left.\Sigma_{2}\right)$ is either the one shown in Fig. 7.30 if $n^{\prime}>n$ or the one in Fig. 7.31 if $n^{\prime}<n$.

\section{FRINGE-SHIFT PATH-DIFFERENCE MEASUREMENTS} (DIFFERENTIAL PROCESS)

A system of straight, parallel and equidistant fringes can be made visible in exactly the same way as with full-duplication microscopes $(\$ 2)$. These fringes are always the loci of equal path differences between the waves $\Sigma_{1}$ and $\Sigma_{2}$. As in full-duplication microscopes,

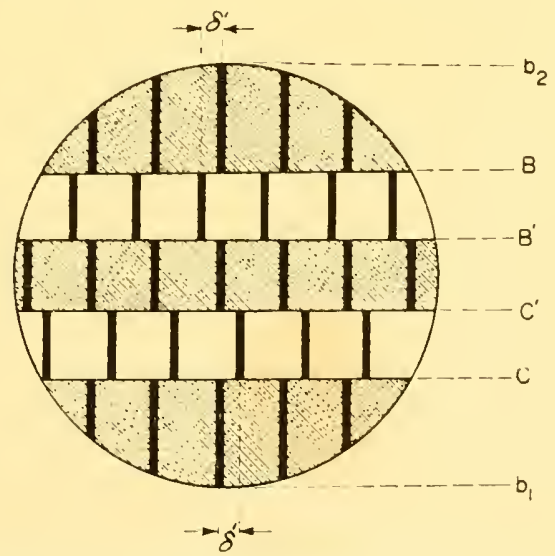

FIG. 7.33. Fringe-shift path-difference measurements in the differential process.

the fringes are paralleled to duplication. The object is so set that the projection of the slope $B B^{\prime}$ on the horizontal plane is paralleled with the fringes. The object in Fig. 7.25 has the aspect shown in Fig. 7.33. The two bands $B B^{\prime}$ and $C C^{\prime}$ are no longer correlated with 
the two duplicated images of the object as in the full-duplication process. In Fig. 7.33, the two areas $B B^{\prime}$ and $C C^{\prime}$ are correlated to the two areas $B B^{\prime}$ and $C C^{\prime}$ of slope $\theta$ on the object in Fig. 7.25. The shift $\partial^{\prime}$ evinces the path difference ad if the latter is less than $\lambda$ and if the shift in relation to the right-hand fringes in the area correlated to $B B^{\prime}$ is included therein. The paih difference $\delta^{\prime}$ may be shown as a fraction $K^{\prime}$ of the space between two fringes. Then:

$$
\delta^{\prime}=\alpha d=K^{\prime} \lambda .
$$

Yet, the actual path difference $\delta$ may equate a whole number $p$ of wave-lengths $\lambda$. plus the fraction $K^{\prime}$ of the space between two fringes. Then:

$$
\delta=a d=p \lambda+\delta^{\prime}=p \lambda+K^{\prime} \lambda=K \lambda .
$$

Provided the dispersions of the substances do not differ overmuch. the procedure to determine $p$ is the same as mentioned in $\S 2$. Let us substitute monochromatic light for white. The dark fringe outside the two strips $B B^{\prime}$ and $C C^{\prime}$ (Fig. 7.33) and the one in either of these two strips are duly located. The micrometer readily affords the means to measure the space between these two dark fringes and ascertain the number of monochromatic-fringe spaces there are within the space. Thus $p$ is faultlessly determined. Observation in white light also

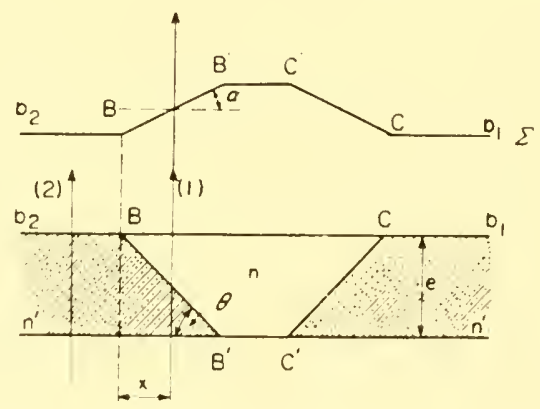

FIG. 7.34. When $n$ is closed to $n^{\prime}$, $\iota$ may be small and $\theta$ large.

evinces whether $n>n^{\prime}$ or $n<n^{\prime}(\$ 5)$. Now, if the slope $\theta$ of the object examined is variable (Fig. 7.34), there is now no need to change over to white light. Let us assume that the slope $B B^{\prime}$ varies continously, in the area $B^{\prime}$, from zero to the value 0 . There is no discontinuity of the slopes between $B^{\prime} C^{\prime}$ and $B B^{\prime}$ and in monochromatic light, the deformation of a specific fringe can be determined and the object's 
slope measured faultlessly. As formerly (\$2) comparing the shift direction of the fringes with the one obtained from a known object shows whether $n>n^{\prime}$ or $n^{\prime}>n$.

\section{DETERMINING THE REFRACTION INDEX FROM PATH-DIFFERENCE MEASUREMENTS}

The first thing to do is to interconnect the slope $a$ of the surface waves to the slope $\theta$ of the object.

Let us revert to the diagrammatic object (Fig. 7.25), shown again in Fig. 7.34. Since $\alpha$ is still assumed to be small (greatly amplified in Fig. 7.34), this means that, provided $\theta$ be large, $n$ is close to $n^{\prime}$. If $e$ is the thickness common to both media, $n$ and $n^{\prime}$, the optical path along the radius (2) is $n^{\prime} e$ and, along the radius (1) located at distance $x$ from $B$, it is $n x \tan \theta+(e-x \tan \theta) n^{\prime}$. The path difference between (1) and $(2)$ is, therefore, $\left(n^{\prime}-n\right) x \tan \theta$. The slope $a$ of the surface wave is derived by merely differentiating $x$ in relation to the expression $\left(n^{\prime}-n\right) x \tan 0$. Then:

$$
\alpha=\left(n^{\prime}-n\right) \tan \theta .
$$

Flat-tints method

Let us substilute $\alpha$ for its value in equations (7.12) and (7.13), when $n^{\prime}>n$, then:

$$
n=n^{\prime}-\frac{b-a}{d \tan \theta}
$$

Therefore $n$ can be determined provided that the object's slope $\theta$ be known. Reverting to the example in $\S 5$ : the tint is blue $(b=0.664 \mu)$ at $B B^{\prime}$ and purple $(a=0.565 \mu)$ at $B b_{2}$. Knowing that $d=25 \mu$, $\tan \theta=0.364\left(\theta=20^{\circ}\right)$ and $n^{\prime}=1.527$, the expression (7.17) gives $n=1 \cdot 52$.

As mentioned in $\$ 3$, accuracy can scarcely exceed 2 places of decimals.

Fringe-shift method

When $n^{\prime}>n$, the expression (7.15) shows:

$$
n=n^{\prime}-\frac{K \lambda}{d \tan \theta} \text {. }
$$

Following up the same numerical example: $d=25 \mu, \tan \theta=0.364$, $n^{\prime}=1.527$ : in mercury green light $(\lambda=0.546 \mu)$ the fringe shift ob- 
served equates $1 / 5$ of the inter-fringe space. Then $K=1 / 5$ whence:

$$
n=1 \cdot 527-\frac{0 \cdot 546}{45 \cdot 5}=1 \cdot 515 \text {. }
$$

Provided that $K$ be measured with an accuracy of 1/40 of the interfringe space and that $\theta=20^{\circ}$ be known within $1^{\circ}$, the index $n$ is determined with an accuracy within two units of three places of decimals.

When the object's slope is unknown

The procedure is the same as described in $\$ 3$. Provided it is feasible, the object of slope $\theta$ is immersed seriatim in two media whose indices, $n_{1}^{\prime}$ and $n_{2}^{\prime}$, are known and, in accordance with the fringeshift method, the shifts $K_{1}$ and $K_{2}$ are evinced. in the media $n_{1}^{\prime}$ and $n_{2}^{\prime}$, respectively, whence the two equations:

$$
n=n_{1}^{\prime}-\frac{K_{1} \lambda}{d \tan \theta} \quad n=n_{2}^{\prime}-\frac{K_{2} \dot{\gamma}}{d \tan \theta}
$$

from which are educed both index and slope:

$$
n=\frac{n_{2}^{\prime} K_{1}-n_{1}^{\prime} K_{2}}{K_{1}-K_{2}} \quad \tan \theta=\frac{\left(K_{1}-K_{2}\right) \gamma_{2}^{\prime}}{\left(n_{1}^{\prime}-n_{2}^{\prime}\right) d} .
$$

\section{MEASURING THE REFRACTION INDEX OF LIQUIDS (DIFFERENTIAL PROCESS)}

\section{With a micro-chamber}

The index $n^{\prime}$ of the prismatic plate $b_{2} B B^{\prime}$ is at a known angle $\theta$ to the plate $a_{1} a_{2}$ (Fig. 7.35). One drop of the liquid of the index $n$

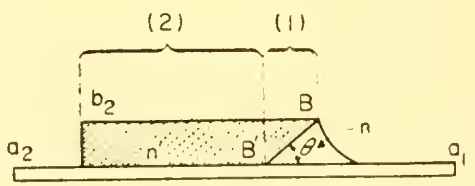

FiG. 7.35. Micro-chamber for index measurements (liquids) in the differential prokess.

to be measured is dropped in the angle $B B^{\prime} a_{1}$ and the plate set upon the interference microscope stage, whereupon the shift $K$ of the fringes observed in the area (1) in relation to those observed in the area (2) is measured. The formula (7.18) gives $n$. To follow the fringes from 
the area (1) to area (2), the tip $B^{\prime}$ in contact with $a_{1}, a_{2}$, may be rounded off ( $\theta$ varies from zero to $\theta$ in the area $\left.B^{\prime}\right)$. There is no need to change over to white light.

\section{Immersion measurement}

In chemical microscopy, the method is suitable for measuring the index of a melted autectic. We have seen that, in Kofler's method, a glass powder of known index is immersed in the liquid (\$ 4). In the differential method, the glass particles are substituted for readily purchasable tiny glass spherules.

Figure 7.36 is a vertical cross-section of the specimen, passing through the centre $O$ of such a spherule of index $n^{\prime}$, radius $r$, and which is immersed in a liquid whose index $n$ is being measured. Let

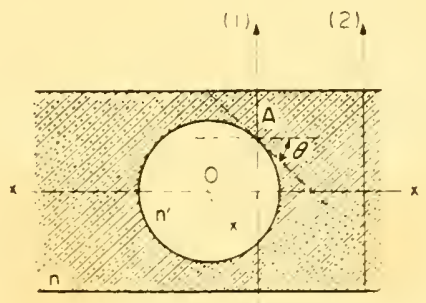

FIG. 7.36. Index measurements by immersion of spherules in the liquid.

us observe the specimen through a microscope whose duplication $d$ is set at right angles to the fringes. The specimen's horizontal view takes on the appearance shown in Fig. 7.37. Since duplication is very weak, it is not shown in Fig. 7.37. It is assumed to be in the direction $X X^{\prime}$. If the indices of both spherule and liquid, $n^{\prime}$ and $n$ respectively, are equal, the fringes within the imaged spherule are spaced as are the outer ones and in alignment with each other.

When $n=n^{\prime}$, all fringes, save the central one, are shifted. Let us measure the shift $\delta$ of one of the fringes along the diameter $X X^{\prime}$ which is at right angles to the fringes. As mentioned previously, the two duplication-originated waves are not shown in Fig. 7.37. Their path difference at random point $A$ along $X X^{\prime}$ is $1+\delta$ and, outside the sphere, $\delta$. A simple calculation shows that:

$$
\delta=2 d\left(n^{\prime}-n\right) \tan \theta .
$$


$\theta$ is readily connected to the sphere radius. Let $O A=x$ (Fig. 7.37) then:

whence:

$$
\tan \theta=\frac{x}{1\left(r^{2}-x^{2}\right)}
$$

$$
\delta=\frac{2 d\left(n^{\prime}-n\right) x}{1\left(r^{2}-x^{2}\right)} .
$$

The observed shift $\delta=K \lambda$ is, in this case, actual as the difference path varies continuously from the centre of the sphere. The previous

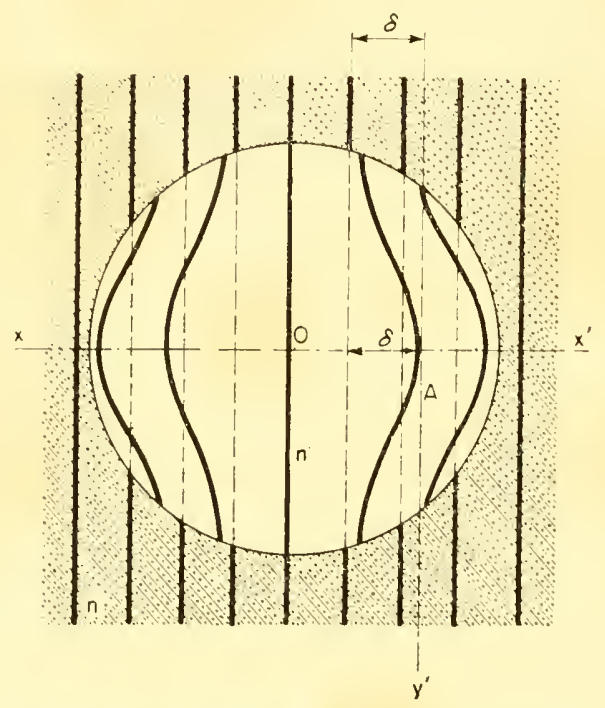

FIG. 7.37. Fringes aspect in the spherule.

formula does not apply, of course, to the edge of the sphere. Granting $m=x / r$ then,

$$
n=n^{\prime}-\frac{K \lambda_{1}\left(1-m^{2}\right)}{2 d m}
$$

There is no need therefore to measure the radius $r$ of the sphere in order to determine the index $n$ : merely knowing $m=x / r$, i.e. the distance $O A=x$ from the point the path difference is measured, is enough. In Fig. 7.37, $K$ is virtually $=4 / 3$ and $O A=x=2 r / 3$, therefore $m=2 / 3$ and:

$$
n=n^{\prime}-\frac{\lambda \cdot 15}{3 d}
$$


If $d=25 \mu, n^{\prime}=1.527, i=0.546 \mu$, then $n=1.509$. Measuring $K$ with an accuracy of $1 / 40$ of the inter-fringe space and if $r$ is known with an accuracy within $r / 40$, then $n$ is deiermined within 5 units of ine fourth place of decimals. To determine whether $n=n^{\prime}$ or $n<n^{\prime}$. a known liquid must be measured.

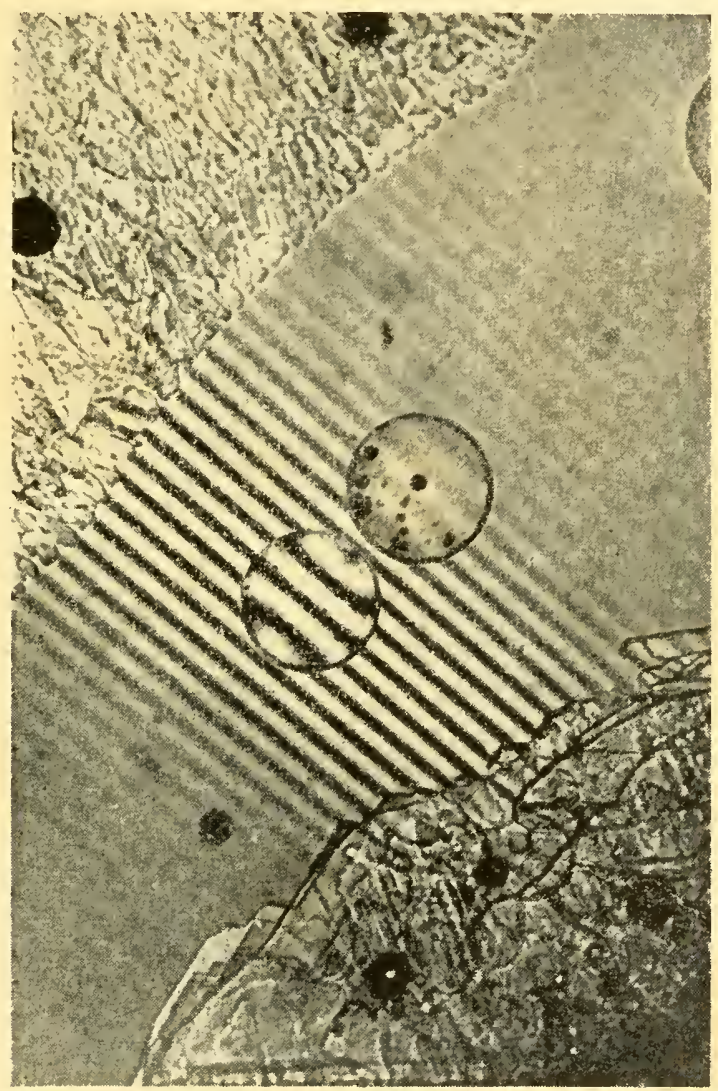

FIG. 7.38. Index measurement of a melted eutectic.

The photograph (Fig. 7.38) shows the method applied to the determination of the index of a melted eutectic. The two unmelted substances which formed the eutectic are visible at the top and bottom of the photograph. The fringe method applied here shows that the fringes in the melted eutectic are not in alignment with the fringes 
within the sphere. At the temperature obtaining when the exposure was made, the indices of both eutectic and sphere were not equal. The eutectic index is educed from the sphere's.

\section{MEASURING BIREFRINGENCE THROUGH A POLARIZING INTERFERENCE MICROSCOPE}

Polarizing interference microscopes are suitable for measuring birefringence of anisotropic substances. This requires the direction of the vibration, passing through the object, to be adequately defined. Interference microscopes in which the light is linearly polarized, just before impinging on the object, are suitable, i.e. the microscopes shown in Figs. 3.19 and 3.24. The instrument, shown in Fig. 3.28, is also adequate, provided the compensating system $W_{1}$ be substituted for a slit. Measurements are also feasible when the polarizer is placed after the object since natural light can be considered as consisting of two incoherent vibrations at right angles. The polarizer only lets through one of the vibrations and phenomena occur as if definitely-directed vibrations were passing through the object.

In a given orientation of the incident wave, an anisotropic object exhibits two rectangular directions in which vibrations may propagate without change. To both these directions corespond two indices $n^{\prime}$ and $n "$. In an anisotropic uniaxial medium only, one of these two indices is constant: the ordinary index, $n_{0}$. The other one fluctuates between the ordinary and the extraordinary index, $n_{0}$ and $n_{e}$, respectively. Birefringence of the medium is the $n_{0}-n_{e}$ difference. Let us take a textile fibre as example: it acts as a uniaxial medium whose optical axis is parallel to the fibre's. Let us assume it were possible to immerse the fibre in a known-index liquid. The procedure to follow is described in $\$ 3$. The fibre index is measured when the fibre is parallel to the polarizer-originated incident vibration, this educing $n_{e}$. The measurement is resumed after rotating the fibre $90^{\circ}$ about its axis so that it is at right angles to the incident vibration, thus educing $n_{e}$. whence the birefringence $n_{0}-n_{e}$.

\section{CONCLUSION}

In the foregoing methods, the measurements shown referred to simple-shape, diagrammatic objects. It follows that such measurements are also applicable to objects of any shape as the measurement may be applied to only a portion of the object. For instance, in the flat- 
tints method (full duplication and differential process), even if the tint varied considerably from one point to another of the object, a small area can be isolated so that its colour does remain virtually constant and detectable. The same applies to the fringe-shift process. Fringes may be substantially deformed, thus making for less accurate performance, but not restraining application of the process. Detecing a dark fringe portion and measuring the shift in relation to the outer fringes of the object will achieve the same result.

The accuracy mentioned in the above examples can readily be improved, provided due regard is given when adjusting the aperture of the incident light-beam (see Chapter VIII, $\$ 2$ ). 


\section{Measuring Thickness and Slopes with Interference Microscopes. Measuring Dry Masses}

\section{TRANSPARENT OBJECTS}

THICKNESS and slope measurements are based on path differences. Therefore all the methods shown in Chapters VI and VII are applicable. As mentioned previously, interference measurements yield the product of an index by a thickness. Hence, to measure the thickness of a rransparent object of index $n$, both its index and that of the medium encompassing it are to be known. Provided the path difference be measured and $n^{\prime}-n$ known, equations (6.1), (7.4), (7.5) and (7.8) yield the thickness $e$ readily.

Likewise, when both the index of a transparent object and that of the medium encompassing it are known, its slope can be determined by the differential method. Equation (7.18) evinces the slope $\theta$ of the transparent object:

$$
\tan \theta=\frac{K i}{\left(n^{\prime}-n\right) d} .
$$

The method was fully set forth in Chapier VII, $\$ 5,6$. Angles of transparent crysial faces can easily be measured in this way even when comparaiively wide, provided that the sample be immersed in a medium whose index is close enough to its own so that the measured path difference $K \lambda$ be small. The differenitial meihod is also applicable to the thickness measurement of an object exhibiting a constant slope $\theta$ (Fig. 8.1). The iransparent object lies at $B C B^{\prime} C^{\prime}$, its index is $n$, and the medium surrounding it is of index $n^{\prime}$. The slope $\theta$ of the face $B B^{\prime}$ is constant. The measurement gives $\theta$ (equation 8.1 ) and, if the width $I$ (projection of $B B^{\prime}$ on a horizontal plane), can be measured by means of a micrometer eyepiece, the thickness $e$ is derived since $e=/ \tan \theta$. 
Multiple-wave devices give excellent thickness-measuring results when low and medium magnificaiion are employed. Owing to the fineness of multiple-wave fringes, very small fringe shifts and, hence,

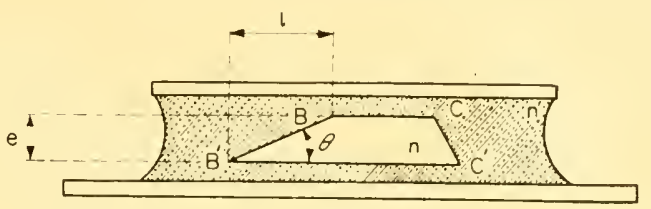

FIG. 8.1. Measuring the thickness of an object exhibiting a constant slope 0 .

small thicknesses, are readily detectable. Measurements are carried out on the same basic principles. The fringe shifi, shown as a fraction of inter-fringe space, evinces the path difference $\left(n^{\prime}-n\right) e$.

\section{REFLECTIVE OBJECTS (THICKNESS MEASUREMENT)}

The interference microscopes, shown in Figs. 4.23, 4.24, 4.26, 4.27 and 4.28, are suitable for measuring the thickness of reflecting opaque objects. The polarizing interference microscopes, shown in Figs. 4.29 and 4.30 , are also suitable provided the double-duplication process be applied.

Measuring a thickness with a two-wave interference microscope

Let us consider, in monochromatic light, the diagrammatic object shown in Fig. 8.2. It consists of a small ridge $A$, of ihickness $e$. The

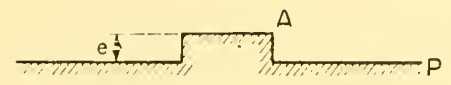

FIG. 8.2. Diagrammatic reflecting object.

microscope shown in Fig. 4.23 is taken as a typical example but, of course, the following is applicable to any microscope based on the lwo-wave interference concept.

The object $P$ is in front of the objective $O_{1}$ (Fig. 8.3) and a reference flat mirror $M$ opposite the oiher objective $O_{1}^{\prime}$. After being reflected by the object $P$ the incident plane wave is deformed and reverts to the objective $O_{1}$ : this wave is shown at $\Sigma_{1}$. Afier being reflected by the mirror $M$, the incident plane wave remains flat and is shown at $\Sigma_{1}^{\prime}$. Both waves are imaged at $P^{\prime}$ where ihey interfere. Directing 
suitably the mirror $M$ enables one to parallel perfectly both waves. Slightly tilting the mirror $M$ causes the wave $\Sigma_{1}^{\prime}$ to be at a small angle with wave $\Sigma_{1}$ (Fig. 8.4) and fringes appear in the field. Aspect is similar to the one shown in Fig. 6.5. The more the relative tilt of

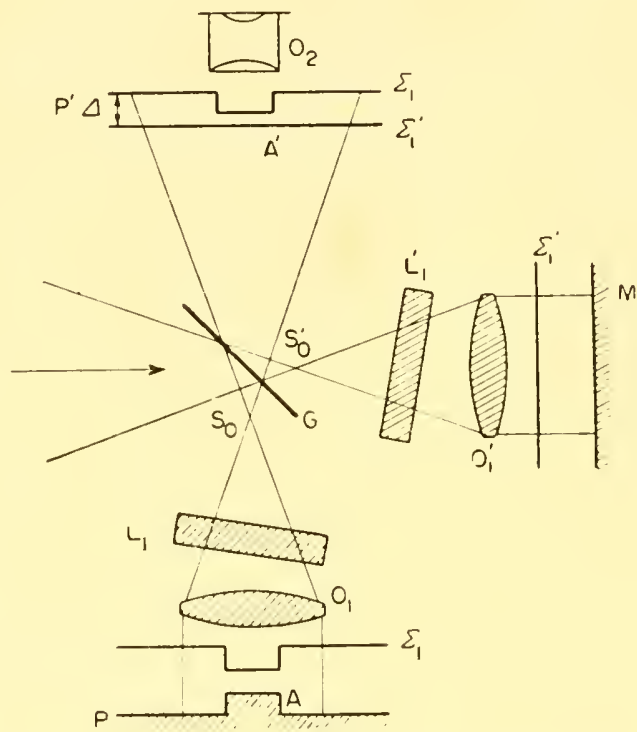

Fig. 8.3. Measuring a thickness with the Linnik-Zeiss interference microscope.

these two waves increases, the closer the fringes. In the Zeiss microscope, the mirror $M$ is not shifted: adjustment of the interference fringes is obtained by tilting the parallel flat plate $L_{1}^{\prime}$. Both the space between fringes and their direction are alterable by tilting $L_{1}^{\prime}$. The purpose of the plate $L_{1}$ is to make the beam paths identical in the

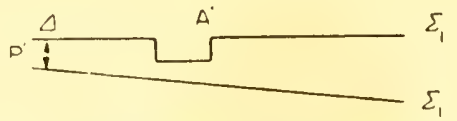

FIG. 8.4. Slightly tilting the mirror $M$ (Fig. 8.3 ) causes the wave $\Sigma$ to be at a small angle to wave $\Sigma_{1}^{\prime}$.

interferometer, this being indispensable for observation in white light. As mentioned before (Chapter VI, $\$ 1$ ), the observed shift $\delta^{\prime}$ does not necessarily represent the actual object-originated path difference $\delta$. In the case depicted in Fig. 6.5 the shift $\delta^{\prime}$ is $\lambda / 3$ approximately but the actual path difference $\delta$ may equate a whole-number times $\lambda$ plus $\lambda / 3$. 
The problem is solved by changing over to white light. If the object is so small that only a portion of the fringe is detected in its image, the white light corresponding to zero path-difference $(1=0)$ is brought on to the object whereupon the location of the white fringe in the field surrounding the object is determined. The distance from the small object itself to the white fringe of the field (outside the object) is shown as monochromatic inter-fringe spaces. Such distance constitutes the path difference. If the object is sufficiently extended as to exhibit at least one fringe, as in Fig. 6.5, merely observing the white fringe, within and outside the object, evinces the path difference which is the inter-fringe distance reckoned as monochromatic inter-fringe spaces.

There remains to determine whether the object $A$ is a prominence or a hollow. Once more, white-light observation gives the clue to the problem. A known object, e.g. a prominence is examined and the position of the white fringe in the field (outside the object) is noted when, concomitantly, this fringe is within the object. The object being in the centre of the field, for instance, it is found that the white fringe (outside the object) is to the left of the field. If the same arrangement is observed with the unknown object then, a prominence is present. Conversely, if the white fringe (outside the object) is to the right of the field, a hollow is involved.

\section{Measuring a thickness with a polarizing interference microscope}

Measuring a thickness is inferred from the preliminary measurement of a path difference. The methods shown in Chapter VII, $\$ \S 1$ and 2, are applicable: the only change required is to substitute the diagrammatic object shown in Fig. 6.1 by the one in Fig. 8.2. The wave re-

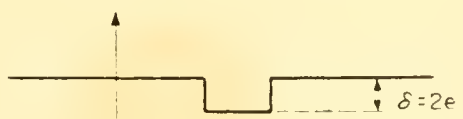

FIG. 8.5. The object reflected wave.

flected by the object, then, has the shape shown in Fig. 8.5 (where a hollow is involved). If $e$ is the thickness of the detail examined, owing to the reflection, the path difference is $\delta=2 e$.

Let us consider the microscope shown in Fig. 4.30. The objectreflected wave, having the shape shown in Fig. 8.5, penetrates into the objective $O_{1}$ and passes through the Wollaston at $J$. This wave 
is split into two waves, $\Sigma_{1}$ and $\Sigma_{2}$ and the imaged arrangement is the one shown in either Fig. 8.6(a) or 8.6(b). If the detail is a prominence. the arrangement is the one shown in either Fig. 8.7(a) or 8.7(b). Provided the Wollaston $W$ be in the focus of the objective $O_{1}$, the flat-tints method is applicable (white light). Let us denote as $A_{1}^{*}$ (Chapter VII, $\$ 1$ )

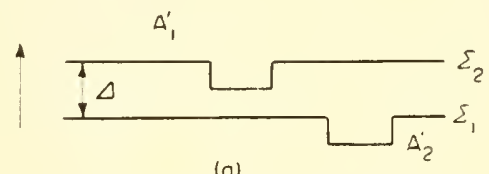

(o)

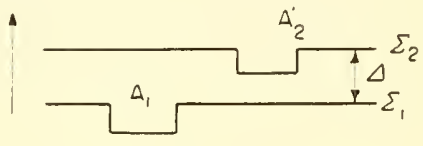

(b)

FIG. 8.6. Arrangement of the two wave surfaces $\Sigma_{1}$ and $\Sigma_{2}$ when the object is a hollow.

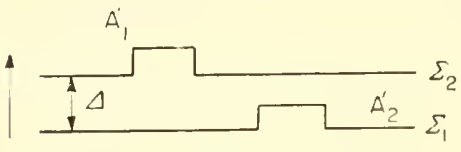

(a)

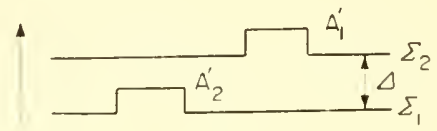

(b)

FIG. 8.7. Arrangement of the two wave surfaces $\Sigma_{1}$ and $\Sigma_{2}$ when the object is a prominence.

the image to the left in the field. There are three colours at $A_{1}^{\prime}, A_{2}^{\prime}$ and the remainder of the field. Assuming that these three tints correspond to path differences whose numerical values (in microns, for instance), educed from Newton's scale, be $a, b, c$ : then (in the case shown in Fig. 8.6(a)):

$$
\begin{aligned}
& \text { at } A_{1}^{\prime} \\
& \text { at } A_{2}^{\prime} \\
& \begin{aligned}
1-2 e & =a \\
1+2 e & =b
\end{aligned} \\
& \Delta=c .
\end{aligned}
$$

Around $A_{1}^{\prime}$ and $A_{2}^{\prime}$

Whence the thickness $e$ :

$$
e=\frac{c-a}{2}=\frac{b-c}{2}
$$

To determine whether the object is a prominence or a hollow, observing an object whose defect is known, e.g. a prominence, will serve the purpose. The arrangement is, therefore, either the one shown in Fig. 8.7 (a) or $8.7(\mathrm{~b})$. Assuming that the left-hand image $A_{1}^{\prime}$ shows a higher tint in Newton's scale than that at $A_{2}^{\prime}$, then the arrangement is the one shown in Fig. 8.7(b). The left-hand image belongs to the lower wave. If the tint of the unknown object imaged at $A_{1}^{\prime}$, is higher than $A_{2}^{\prime}$ it is, likewise, a prominence. If it is the tint of $A_{2}^{\prime}$ which is higher. then the object is a hollow. 
Fringes appear as the Wollaston $W$ (Fig. 4.30) is moved away from the objective in relation to its former position, e.g. when no longer in the focus. Then the thickness $e$ can be measured by the fringe method in monochromatic light (Chapter VII, § 2). The wave arrangement is similar to the one depicted in Fig. 7.9. Assuming that the detail observed (either prominence or hollow) has the shape of a small rectangle, the appearance of the field is identical with the one shown in Fig. 7.10. The observed shift $\delta^{\prime}$ does not necessarily represent the actual path difference $\delta=2 e$. We have already seen that the actual shift may equate a whole-number times the wave-length of the light used, that is, $p \lambda$ plus the portion $K^{\prime}$ observed.

Now, equation (7.8) is written thus:

$$
\delta=2 e=p \hat{\lambda}+\delta^{\prime}=p \hat{\lambda}+K^{\prime} \lambda=K \lambda .
$$

This equation yields the thickness $e$ of the detail observed. To determine the factor $p$ it is necessary to change over to white light, in accordance with the process described in Chapter VII, $\S 2$. No problem

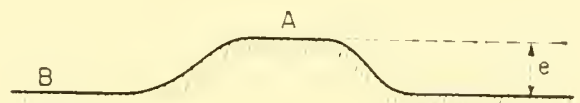

FIG. 8.8. Diagrammatic object, the thickness of which varies continuously.

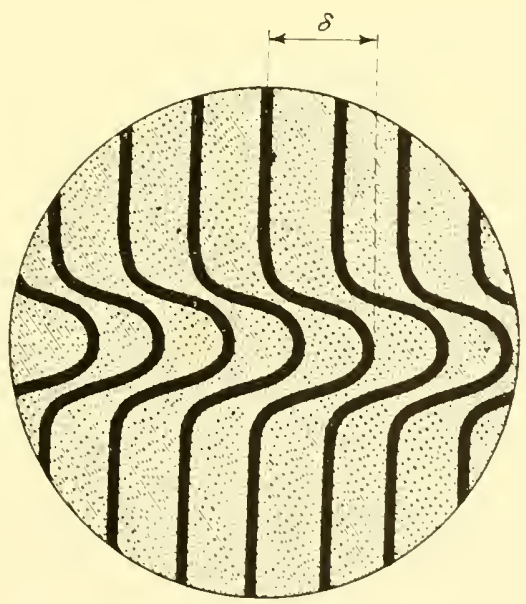

FIG. 8.9. There is no uncertainty in measurements when the thickness varies continuously: the shift $\delta$ observed is the actual shift. 
is involved when the thickness of the detail varies continuously as, for instance, in the case of the object depicted in Fig. 8.8. When changing over from the area $B$ to the area $A$, the path difference variation is continuous and all that is required is to follow up the deformation of a specific fringe (see Chapter VI, $\$ 1$ ). Assuming that the object shown in cross-section (Fig. 8.8) has the shape of a small extended strip perpendicular to the plane of the figure, the appearance is the one shown in Fig. 8.9. There is no uncertainty as to the shift of fringes and the shift $\delta$ observed in Fig. 8.9 is the actual shift evincing the thickness $e$ of the object derived from the equation (8.4).

\section{Measuring a thickness with the multi-wave interference microscope}

Changing over to white light, in multi-wave microscopes, is not feasible. Therefore, if the object being measured originates a substantial fringe shift, there are no means to measure the actual value of the path difference in white light. Nevertheless, in some cases, the problem is solved by observing the phenomena with two monochromatic radiations. It follows that such a difficulty is only present when there is a break between the fringes within and around the object. Such is the case in the object shown in Fig. 8.2. Provided it is ascertained that the thickness $e$ of the object is small in relation to $\lambda$, the observed shift $\delta$ is the actual shift. Such being the case, the thickness $e$ of the object is derived by expressing the shift as a portion $K^{\prime}$ of one inter-fringe space.

Then:

$$
\delta^{\prime}=K^{\prime} \lambda=2 e
$$

whence the thickness $e$.

There is, likewise, no problem in the present instance provided the thickness of the detail varies continuously in relation to the field surrounding it: merely following up the deformation of a specific fringe will serve the purpose. The appearance of the field is very similar to the one shown in Fig. 8.9 but the fringes are very narrow.

\section{Measurement accuracy}

No mention has yet been made about a prominent factor: the N.A. of the incident light-beam. It is granted that the path difference, following reflection or after passing through the transparent object is clearly determined as $2 e$ and $\left(n-n^{\prime}\right) e$, respectively. These values square with path differences when the incident light-beam consists 
of parallel beam (pin-point source at the condenser focus) which is normal to the specimen. To obtain sharper images, opening the condenser diaphragm is always beneficial. Under such conditions, the specimen is illuminated by numerous parallel-ray beams, slanting at various angles. In Fig. 8.10 the diaphragm $D$ of the condenser $C$ is reduced to the very low aperture $S_{0}$, located in the focus of $C$.

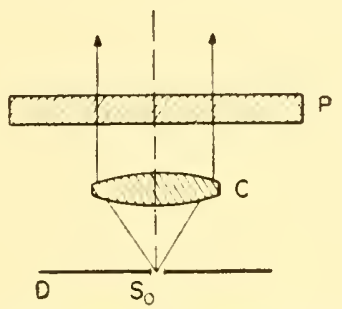

FIG. 8.10. The specimen is illuminated by a parallel beam (low condenser aperture).

The specimen is illuminated by a parallel beam. In the case shown in Fig. 8.11, when the diaphragm $D$ is opened to a greater extent, any point $S$ of the diaphragm aperture sends forth, through the specimen, a parallel-ray beam whose slant $\alpha_{0}$ varies as does the position of point $S$. Phenomena are identical in reflected light (Fig. 8.12). If the diaphragm of the vertical illuminator (not shown in Fig. 8.12)

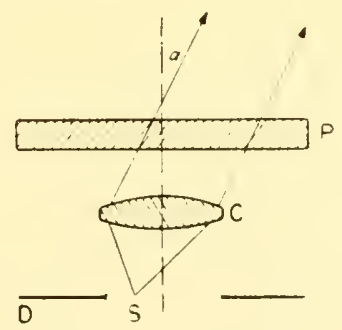

FIG. 8.11. The specimen is illuminated by a parallel beam whose slant $\alpha$ varies as does the position (diaphragm $D$ opened).

is almost closed, phenomena occur as if there were a pin-point radiant $S_{0}$ at the focus of the objective $O_{1}$. The specimen $P$ is illuminated by a parallel-ray beam. If the diaphragm of the vertical illuminator is opened still further the imaged source does not consist of a point at $S_{0}$ but of an extended area. A random point $S$ of this image sends a beam, of slant $\alpha_{0}$ on to the reflective object $P$. Assuming $\alpha$ be the maximal value of $\alpha_{0}$ in Fig. 8.11 and 8.12, it is the slant of the beam when the 
point $S$ is on the edge of the imaged source. The aperture $a$ of the light-beam is an essential factor in measurement accuracy.

Let us consider, for instance, the thickness of a small object in Linnik's interference microscope (Fig. 8.3). The instrument is adjusted

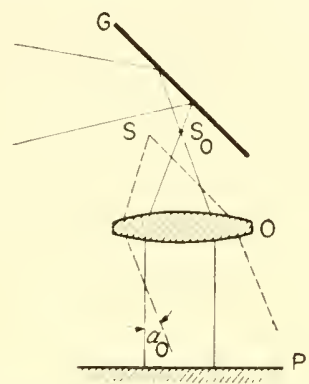

FiG. 8.12. Reflected light: a random point $S$ sends a beam whose slant $\alpha$ varies as does the position of $S$.

to give rise to fringes in the field the thickness of the object being measured by shifting the fringes. Estimating the inter-fringe spaces provides the answer. Let us assume that the images $S_{0}$ and $S_{0}^{\prime}$ of the light-source (not shown in Fig. 8.3) are pin-points on the axes of the

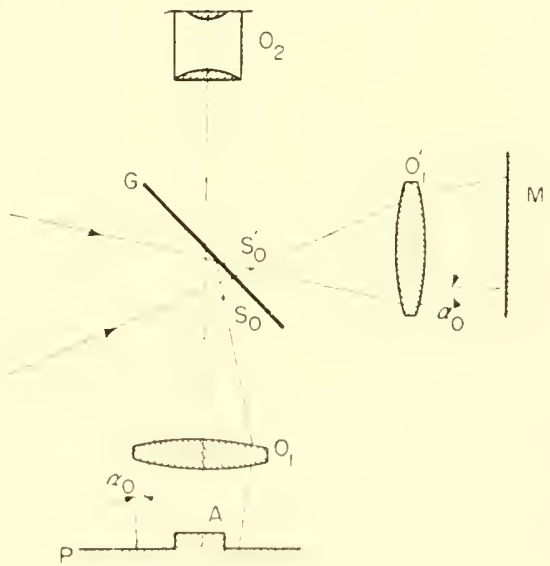

FIG. 8.13. The beams are not normal to the surfaces $M$ and $P$.

objectives $O_{1}$ and $O_{1}^{\prime}$. The object is illuminated by a parallel-ray beam normal to the specimen $P$. If $e$ is the thickness of the detail examined, the path difference is $\delta=2 e$ and the fringe inter-space is $\lambda / 2$. When 
changing over from one fringe to the next, thickness increases or decreases by $\lambda / 2$. This is no longer true if shifting the source moves the images $S_{0}$ and $S_{0}^{\prime}$ away from the axis. Nor is the beam perpendicular any longer: its slant is a (Fig. 8.13). The inter-space fringe then equates $\lambda /(2 \cos \alpha)$. If a non-pinpoint source is now considered, a system of fringes is correlated to every element of the source. Inter-fringe spacing changes from one system to another. Finally. owing to these phenomena adding up, two occurrences take place:

(a) The fringe inter-space observed is no longer $\lambda / 2$, thus giving rise to errors in path-difference measurements;

(b) Fringe contrast drops rapidly as path-difference increases.

It may be taken for granted that the path differences considered are always small enough so that fringe contrast does not drop substantially. For instance, an illuminating aperture $\sin \alpha=0.60$ does not materially affect contrast if the path difference does not exceed approximately four wave-lengths (Dyson).

Tolmon and Wood, Gates, Bruce and Thornton have investigated, in reflecting objects, the effects of the angle $\alpha$ on fringe inter-spacing. The relevant data are tabulated hereunder:

\begin{tabular}{c|c|c|c}
\hline $\begin{array}{c}\text { Accuracy } \rightarrow \\
\begin{array}{c}\text { Aperture of incident } \\
\text { beam } \downarrow\end{array}\end{array}$ & $\frac{\lambda}{10}$ & $\frac{i}{20}$ & $\frac{\lambda}{100}$ \\
\hline $\sin \alpha=0.10$ & & $\delta=12 \lambda$ & $\delta=3 \lambda$ \\
\hline $\sin \alpha=0.20$ & $\delta=10 \lambda$ & $\delta=4 \lambda$ & $\delta=\lambda$ \\
\hline $\sin \alpha=0.30$ & $\delta=4 \lambda$ & $\delta=2 \lambda$ & \\
\hline
\end{tabular}

The above table does not show the data corrections needed to secure exact measurements. It evinces for every object exhibiting the path difference $\delta$, the aperture $\alpha$ of the incident beam not to be exceeded in order to attain a measuring accuracy within $\lambda / 10, \lambda / 20$ or $\lambda / 100$. For instance, it is required to measure a reflecting object, whose thickness $e$ gives rise to a path difference $\delta=2 e=3 \lambda$, within $\lambda / 100$. The illuminating aperture should be reduced to the value $\sin \alpha<0 \cdot 10$. As regards transparent objects, the path difference is $\left(n-n^{\prime}\right) e$ in normal incidence, and $n$ and $n^{\prime}$ are then to be included in the calculation. 
E. Ingelstam and L. P. Johansson have investigated the point at issue both theoretically and experimentally. When $n=1.50$ and $n^{\prime}=1$, they found that a numerical aperture close to 0.25 provided accuracy within $\lambda / 100$ if the object-originated path difference did not exceed $\lambda$. An aperture higher than $0 \cdot 15$ is not feasible if the same accuracy is to be achieved with a $2 \lambda$ path difference.

Therefore, if highly accurate measurements are required, the aperture should be kept at values as low as image sharpness will allow.

Two incompatible view-points stem from the foregoing when accuracy in thickness and accuracy in width are both required simultaneously (Ingelstam). If thickness accuracy (path difference) is the paramount consideration, this can only be obtained at the expense of transverse measurement accuracy, i.e. less definition.

\section{MEASURING ANGLES OF CONTACT BETWEEN LIOUID AND SOLID SURFACES}

Formerly, when investigating the wetting of a solid, researchers endeavoured to measure direct the contact angle, i.e. the angle formed by the solid with the liquid surface. Accurate measurements were difficult to secure with the instruments employed at the time and have been replaced by more easily carried out dynamic measurements.

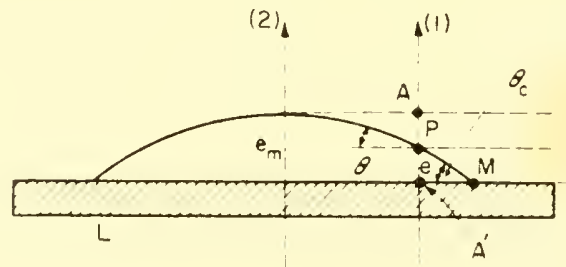

FiG. 8.14. Drop of transparent liquid on a flat horizontal surface.

The differential method, described in Chapter VIII, is so readily applied that contact-angle measurements are revived as they can be carried out in the very small areas discernible in a microscope. Angleof-contact variations, originated from various causes, can thus be gone into, step by step, with great accuracy.

Figure 8.14 shows a drop of transparent liquid on a flat horizontal surface and Fig. 8.15 the meniscus formed by contact with a vertical solid wall. 
In the first instance, the plate $L$ is assumed to be transparent and the whole arrangement placed on the stage of a polarizing interference microscope. The differential method is used. The liquid drop may also be put on the reflecting plate $L$. Phenomena are then observed by reflection although the principle of the method is unaltered. In the second instance (Fig. 8.15) the liquid is enclosed in a micro-chamber, set on the microscope stage, the plate $L$ being immersed vertically.

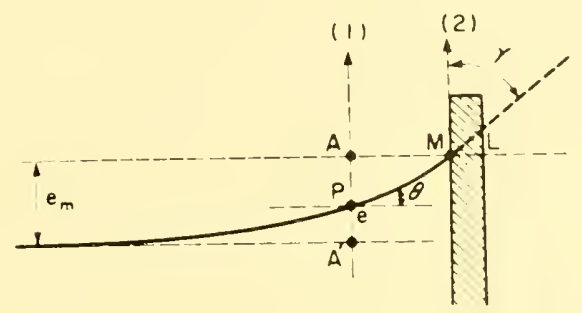

FIG. 8.15. Solid plate $L$ immersed in a liquid.

Let us consider, for instance, Fig. 8.14. The angle $\theta$ is the tangent angle with the horizontal line $A^{\prime} M$ at the surface of the liquid. The thickness of the liquid drop, in the area through which the ray (1) passes is $e$. The thickness $e_{m}$ is maximum in the area (2). $n$ being the index of the liquid, the optical path between $A$ and $A^{\prime}$ is $J_{0}$ $=(n-1) e+e_{m}$. Assuming the angle $\theta$ to be small, then, according to equation (7.16)

$$
\alpha=(n-1) \theta .
$$

The angle $\alpha$ is the slope of the transmilted surface wave $\Sigma$ (Fig. 8.16). According to equation (7.13), then

$$
\theta=\frac{b-a}{(n-1) d} .
$$

The tint at $P$ is determined, which yields $b$, and $a$ is derived by determining the tint outside the drop. The contact angle $\theta_{c}$ sought is derived by measuring $b$ quite close to $M$. When a solid plate is immersed (Fig. 8.15) the contact angle is $\gamma$ and then, $\gamma=\pi / 2-\theta$.

Let us now assume that, in the flat areas outside the drop (Fig. 8.14), the instrument is so adjusted as to show the first order purple $(0.565 \mu)$. If the second order sky blue is observed at $M(0.664 \mu)$, then $b-a$ $=0.099 \mu$. To compute $\theta$ in formula (8.7) the data required are the duplication $d$, characteristic of the interference microscope and the 
index $n$ of the liquid. Applying the fringe-shift method yields a more accurate measurement of $b-a$ (Chapter VIII, $\S$ ). Figure 8.17 shows schematically the fringe arrangement whose direction is assumed to be parallel to duplication. Let us consider the point $A$ where the tangent of the edge of the drop is perpendicular to the fringes. This

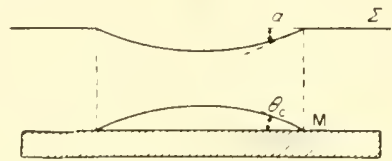

FIG. 8.16. Transmitted wave surface 2 .

shows at once the contact angle at $A$. When changing over from the edge of the drop $A$ to the area outside it but, nevertheless, quite close to $A$, the path difference varies by $\lambda / 2$ in Fig. 8.17. As mentioned previcusly, the whole of the interference sequence is determined by locating the dark-fringe shift in white light. If the actual path difference is the observed $\lambda / 2$ path difference, then, $b-a=\lambda / 2$.

To determine the contact angle at a point $M$ other than $A$ or $B$. the duplication value in the $M M^{\prime}$ direction is to be included in the

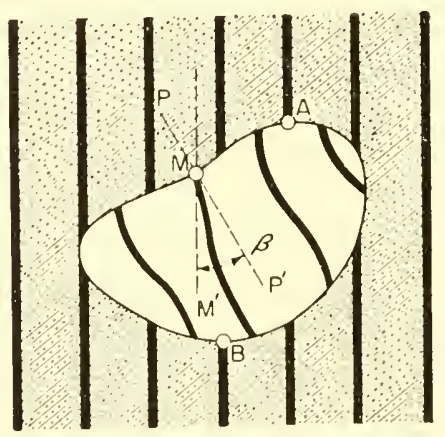

Fig. 8.17. Contact angle measurement with the fringes process.

equation (8.7) (see Chapter VIII, $\$$ ). If $\beta$ is the angle formed by $M M^{\prime}$ with the steepest line $P P^{\prime}$ at $M$, then:

$$
0=\frac{b-a}{(n-1) d \cos \beta} .
$$

Merely rotating the drop in its holder on the stage so that the position previously held by $A$ be now that of $M$ will obviate the $\cos \beta$ 
factor. Figure 8.18 shows, using the fringes method, the photograph of a drop of water deposited on a not thoroughly clean glass plate.

The method is applicable to the study of small insects, such as "flying" arthropoda. Such insects float on the water because they are

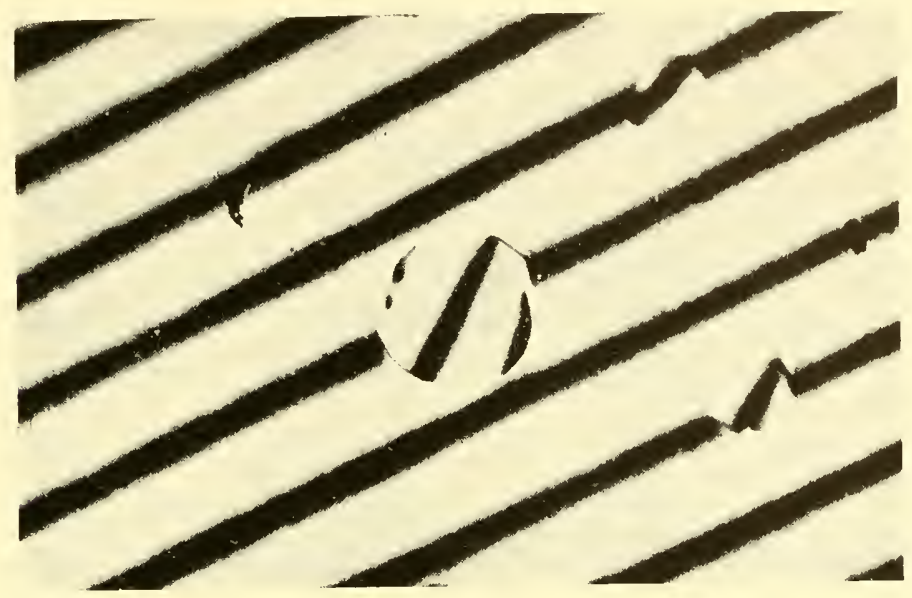

FIG. 8.18. Drop of water deposited on a not thoroughly clean glass plate.

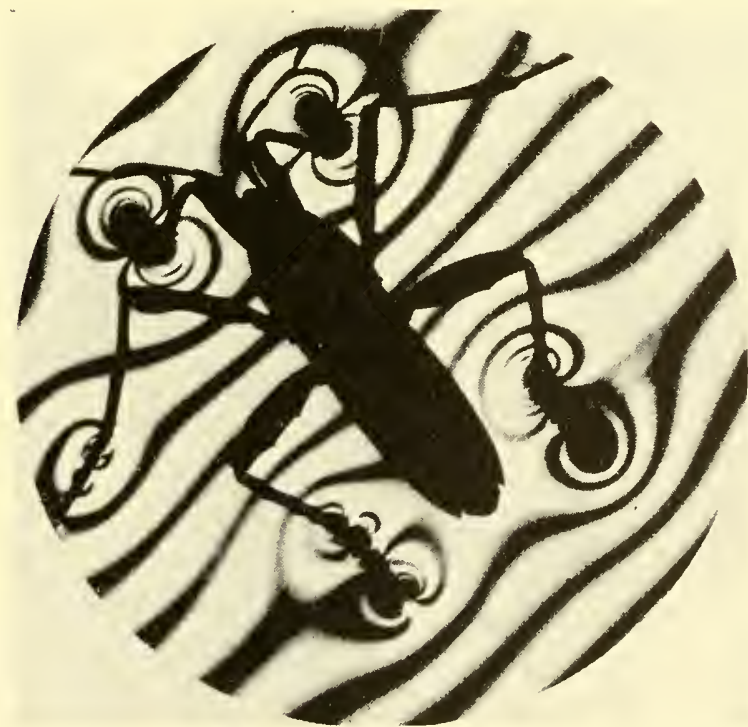

FIG. 8.19. Water surface deformation produced by a small insect (Velia). The deformations are observed using the fringes method. 
not wet. The portion of their body in contact with the water flattens down the surface of the liquid and the contact angles so formed are measurable. The flat-tints method originates vivid colours in the areas where the surface of the water is flattened down by the insect's contact. Figure 8.19 shows a "Velia" about $7 \mathrm{~mm}$ long. The water-surface distortions are observed using the fringes method.

\section{MEASURING DRY MASSES BY MEANS OF AN INTERFERENCE MICROSCOPE}

R. Barer, H. G. Davies and M. H. F. Wilkins have suggested applying interference methods to the measurement of living-cell dry masses. Let us consider a diagrammatic cell consisting of a small plate of thickness $e$ and surface $s$ (Fig. 8.20). The cell is of a solid

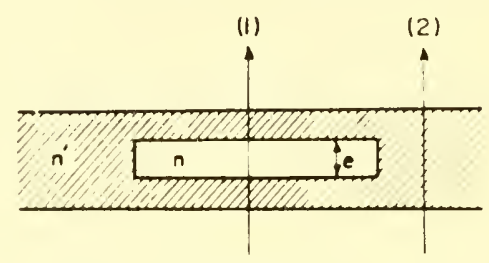

FIG. 8.20. Diagrammatic cell.

substance of total dry mass $M$ and a liquid substance of index $n^{\prime}$. The cell is also surrounded by the selfsame liquid. Where living cells are involved, the index $n^{\prime}$ selected may well be that of water (1.33). The refraction index of the cell, different from $n^{\prime}$, equates $n$. Let us denote the cell's concentration, i.e. the number of grammes of solid substance it holds per $\mathrm{cm}^{3}$, then, the dry mass of the cell is:

$$
M=\text { cse. }
$$

Let us write:

$$
K=\frac{n-n^{\prime}}{100 c} .
$$

The value of $K$ is 0.0018 for proteins, 0.0017 for lipoprotein and $0.0017-0.0020$ for the two types of nucleic acid. Hence in measuring the dry mass of cells and other tissue elements composed mainly of these substances the value 0.0018 may be selected for $K$ (errors will not exceed about 10 per cent). $K$ is a constant for a specific substance. 
Equations (8.9) and (8.10) yield:

$$
M=\left(n-n^{\prime}\right) e \frac{s}{100 K} .
$$

Now, $\left(n-n^{\prime}\right) e$ equates the path difference $\delta$ between a light ray (1) passing through the cell and another ray passing next to it. Then:

$$
M=\frac{\delta s}{100 K} .
$$

Since the constant $K$ is known, the dry mass of the cell can be derived by measuring its surface $s$ and the path difference $\delta$ it originates in relation to the medium encompassing it. The measurement of $\delta$ is made by a two-wave interference microscope, e.g. Dyson`s or a fullduplication polarizing interference microscope. The method applies to uniform objects within which $\delta$ has a well-defined constant value. However, measuring the dry mass of a non-uniform object is feasible by observing the zones where $\delta$ is constant. The dry mass and area of such zones is computed by means of formula (8.12) and the total dry mass is derived by adding the elementary dry masses. $\delta=f(x, y)$, showing the $\delta$ variations in terms of the coordinates $x$ and $y$ of a point of the cell's surface can be plotted. The total dry mass is derived by computing graphically the integral:

$$
M=\iint \frac{f(x, y)}{100 K} d x d y .
$$

The measurements carried out using this method apply likewise to transparent objects enclosing no liquid. Reverting to Fig. 8.20, the object of thickness $e$ and index $n$ has a density $\varrho$ and a mass $M=$ soe. Since the path difference originated by the object is $\delta=\left(n-n^{\prime}\right) e$, then:

$$
M=\frac{\delta s}{\left(n-n^{\prime}\right) \varrho} .
$$

Provided the following be known: the index $n$, the area $s$ and density $\varrho$ of the object and the index $n^{\prime}$ surrounding it, its mass $M$ is determined by measuring, with an interference microscope, the path difference $\delta$ it gives rise to. 


\section{CHAPTER IX}

\section{Infra-red and Ultra-violet Microscopy}

INFRA-RED and ultra-violet microscopy is a powerful means of investigation which finds numerous applications. It is well known that there are many objects, transparent in visible light, which have absorption bands in the infra-red and the ultra-violet. In order to make the object visible in the receiver, merely using a radiation whose wave-length corresponds to one of the object's absorption bands will achieve the result. For instance, living cells, transparent in visible light, exhibit details in the ultra-violet because the $2700 \AA$ wave-length radiations are absorbed by the nucleic acids. Moreover, ultra-violet light enables reduction of the diffraction-disk diameter (Chapter I, equation 1) thus improving resolving power. Lastly, some substances. under the effects of ultra-violet rays, radiate some visible light whose colour frequently helps in identifying or discriminating these substances.

\section{MICROSCOPE OBJECTIVES IN INFRA-RED AND ULTRA-VIOLET MICROSCOPES}

Up to a wave-length of about $1 \cdot 5 \mu$, standard achromatic objectives may be used but the image quality is not too satisfactory owing to the chromatic variation of spherical aberration. This aberration is more particularly prominent in powerful achromatic objectives. Better results are evidenced, in general, by apochromatic objectives whose residual aberrations are not so marked. Designing glass achromatic objectives transparent up to $3 \mu$ and even $10 \mu$ is feasible provided that they be applied to a narrow spectral range. In fact, there are several optical glasses, currently used, which are transparent up to $3 \mu$. Beyond, special glasses or artificial crystals are required.

Nevertheless, from a $1.5 \mu$ wave-length approximately, application of reflecting objectives is a suitable process. Such objectives are 
perfectly achromatic thus ensuring image focusing in visible light. They also have a long working distance. An objective of numerical aperture 0.65 has a working distance of approximately $13 \mathrm{~mm}$ whereas that of a lens objective does not exceed $0.5 \mathrm{~mm}$ for the same aperture. Therefore phenomena can be observed with a magnifying power unattainable with lens objectives. Such is the case, for instance, when observing an inaccessible object through the peep-hole of a furnace.

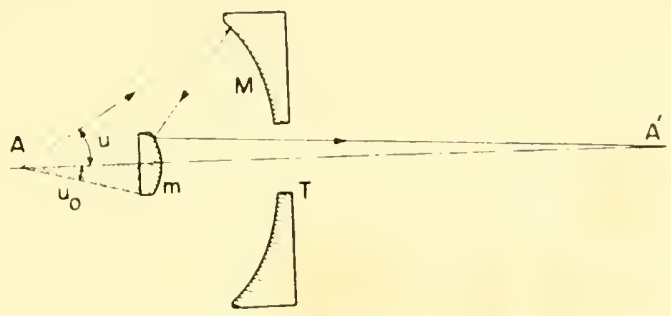

FIG. 9.1. Schwarzschild-type reflecting objective.

Reflecting objectives are based on the two types of assembly shown in Figs. 9.1 and 9.2. In the objective shown in Fig. 9.1 (Schwarzschildtype objective), the light diffracted by the object $A$ is first reflected, by the concave spherical mirror $M$, then by the convex spherical mirror $m$, on to the image $A^{\prime}$ through the aperture $T$, provided in the mirror $M$. In the objective shown in Fig. 9.2 (Cassegrain or Newton type) the light is reflected either first on a flat or convex mirror $m$

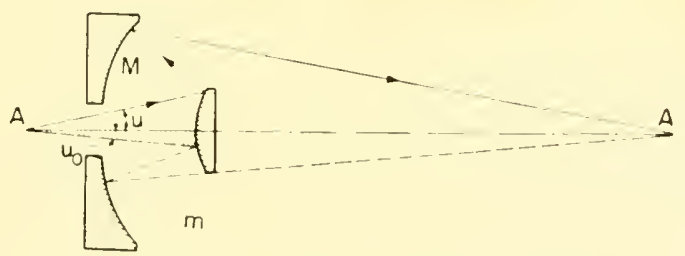

FIG. 9.2. Cassegrain type reflecting objeclive.

and then on the concave mirror $M$. In both arrangements, the rays located in the half-cone angle $u_{0}$ are stopped and occlusion of a portion of the incident beam occurs. The occlusion ratio of a mirror objective is defined as follows: $\sin u_{0} / \sin u$. This ratio is higher in objectives of the second type and is the reason why Schwarzschild-type objectives are now preferred. 
C. R. Burch has built Schwarzschild-type objectives which are, as yet, the best ever designed. He showed that, if both mirrors are spherical, the objective can be corrected from both spherical aberration and coma but that occlusion of the incident beam by the small mirror cannot be less than 45 per cent. Nor can the numerical aperture of an objective comprising two spherical mirrors exceed 0.50 approximately (although it can be increased to 0.60 in the lower infra-red up to $1 \mu$, and $0 \cdot 80$ above $2 \cdot 5 \mu$ ).

The numerical aperture can be increased by retaining the sphericalaberration correction but coma develops and the field becomes very small. With an objective of numerical aperture 0.65 , the field diameter does not exceed $10 \mu$ at $2500 \AA$.

In Chapter I, $\$ 3$, it was mentioned that the central occlusion brought about by the small mirror altered the diffraction disk and reduced the diameter of Airy's disk while retaining the zero-minimum rings and making the bright rings definitely stronger. Such increased intensity of the bright rings lessens image contrast. If the occluded area does not exceed 10 per cent of the total wave area in the space image (see Chapter I, $\$ 1$ and Fig. 1.7), the diffraction phenomenon is but slightly altered, this corresponding to an occlusion ratio $\sin u_{0} / \sin u$ of approximately $0 \cdot 30$. Using a non-spherical surface (the mirror $M$ ), Burch reduced occlusion to 4 per cent. When both surfaces are nonspherical, the numerical aperture 0.65 is increased to 0.95 . The N.A. can still be increased further by setting on the object a plano-convex lens whose centre is in coincidence with the object. In this way the objective of N.A. 0.65 (dry-front-lens) becomes an objective of N.A. 0.98 and, with immersion, the dry-front-lens objective of N.A. 0.95 becomes an objective of N.A. 1.4 .

Objectives comprising 2 spherical mirrors have been used by E. R. Blout, G. R. Bird and D. S. Grey for infra-red micro-spectroscopy. The objectives were of N.A. 0.63. Setting on the object a silver-chloride or a thallium-bromide-iodide lens increased the N.A. to 1.25 and $1 \cdot 50$, respectively. This arrangement gives good results in the range 2 to $20 \mu$.

S. Miyata, S. Yanagawa and S. Noma have evolved non-spherical concave mirror objectives by evaporating in iacuo, on a spherical mirror, a layer of zinc sulphide of variable thickness.

Mirror objectives are also employed in the ultra-violet. W. Thornburg has developed a reflecting objective, featuring a low occlusion- 
ratio and long working distance (Fig. 9.3), designed for ultra-violet microspectroscopy.

The rays issuing from the object $A$ are reflected by the flat mirror $m_{1}$ on to which they revert after being reflected by the concave mirror $M$. Next, they are reflected once more by the convex mirror $m_{2}$ and end

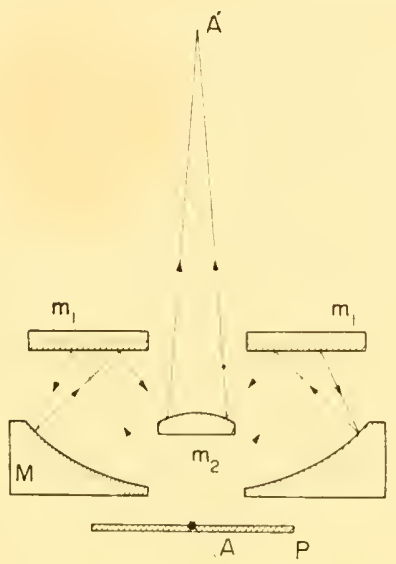

FIG. 9.3. Thornburg reflecting objective.

in the image $A^{\prime}$. The residual spherical aberration can be corrected by altering the shape of the surface $m_{1}$. The N.A. of this objective is 0.77 (dry front lens), its occlusion ratio 0.34 and magnifying power $\times 100$.

Objectives which are both reflecting and refracting have also been devised. Such arrangements comprise one or two mirrors added to refracting elements which provide correction of aberrations without having recourse to non-spherical surfaces. They usually comprise a fairly high number of air-glass surfaces which may promote origination of stray light but, against this, they have a low occlusion ratio and an extensive field. Using a quartz meniscus one face of which is aluminized, B. K. Johnson evolved a high N.A. objective (Fig. 9.4). The rays from the object $A$ pass through the lens $l_{1}$ (fused quartz) and are reflected on the $45^{\circ}$-slanted plate $G$. After passing through the lens $l_{2}$, they are reflected from its aluminized back to end in image $A^{\prime}$. The back of $G$ should be coated in order to prevent a double-image. As shown in Fig. 9.4, the N.A. of the objective is 0.84 which can be increased to 1.27 by adding a meniscus lens above $l_{1}$. When the objective is focused for a spectral line in visible 
light, it is equally well focused for a spectral line in the ultra-violet. Furthermore, the spherical aberration of the arrangement is well corrected both in visible and ultra-violet light. Lastly, owing to the

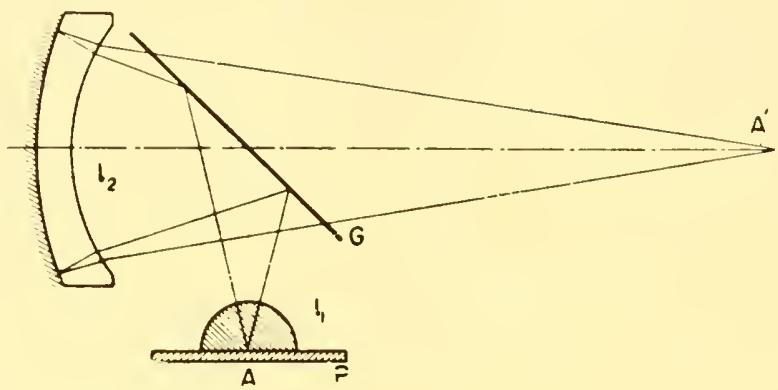

FIG. 9.4. Johnson reflecting objective.

$45^{\circ}$-slanted plate $G$, the light-beams are not occluded. B. K. Johnson's system acts as a spherical mirror whose aberrations are corrected by a correcting lens.

Bouwers has also used correcting meniscus lenses the study of which was carried out by D. D. Maksutov (Fig. 9.5). The light from

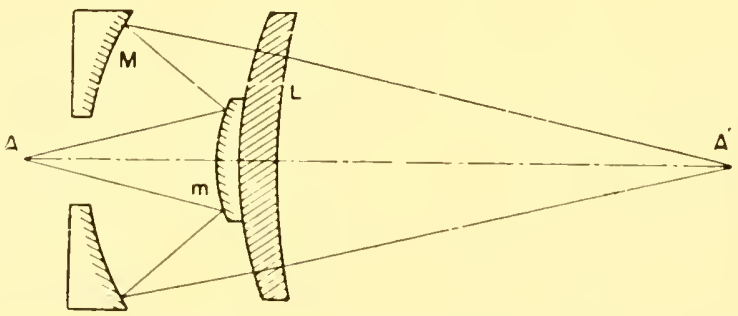

FIG. 9.5. Reflecting objective with correcting lens $L$ (D. D. Maksutov).

the object $A$ is first reflected by the mirrors $m$ and $M$, then passes through the correcting lens $L$ (meniscus) and ends in the image $A^{\prime}$.

D. S. Grey has described Schwarzschild-type objectives comprising many refracting elements. The objective shown in Fig. 9.6 is corrected from $2200 \AA$ up to the lower infra-red range, it has a N.A. $1 \cdot 1$ and an occlusion ratio of approximately $0 \cdot 26$. The refracting elements between the object and the mirror $m$ enable one to increase the N.A. while those between $M$ and $m$ increase the magnifying power and provide improved correction. 
In 1950, D. S. Grey described several objectives for ultra-violet applications. Numerical apertures range from 0.4 to $1 \cdot 0$. Figure 9.7 shows schematically one of these objectives. The N.A. is 1.0 and

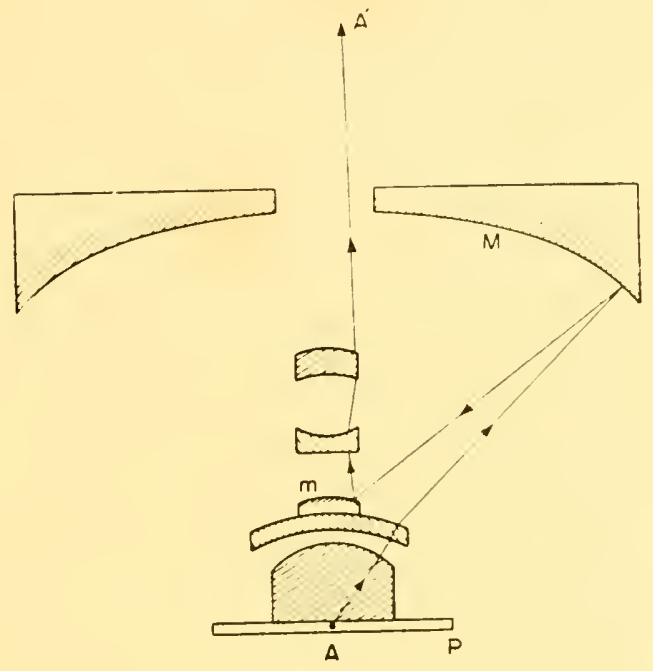

FiG. 9.6. Grey and Lee reflecting objective $\left(n \sin u=1,1 \quad \frac{\sin u_{0}}{\sin u}=0 \cdot 26\right)$.
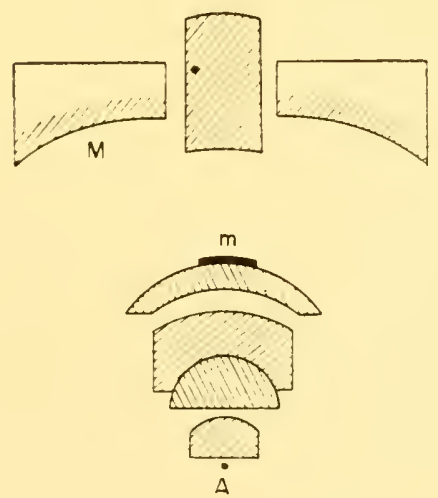

FIG. 9.7. Grey reflecting objective $\left(n \sin u=1 \quad \frac{\sin u_{0}}{\sin u}=0 \cdot 30\right)$.

the occlusion ratio 0.30 ; the convex mirror consists of an aluminized portion of the convex surface of a lens.

Nomarski and Bernstein devised an objective of N.A. $n \sin u=0.90$, magnifying power $\times 100$ and spherical aberration-corrected in the 
2300 to $4360 \AA$ range (Fig. 9.8). The objective is of the immersion type and comprises a hemispherical lens (1). The intermediate image $A_{1}^{\prime}$ is formed after reflection has taken place on the two reflecting elements (2) and (3) and, next, travels through the two doublets (4)
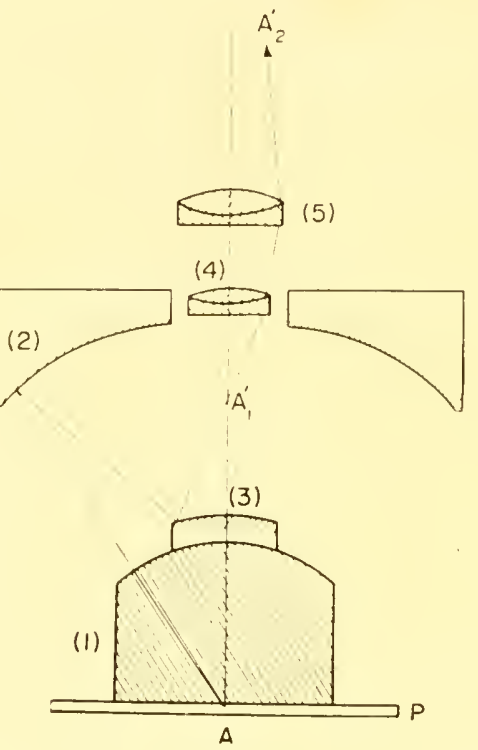

FIG. 9.8. Nomarski and Bernstein reflecting objective.

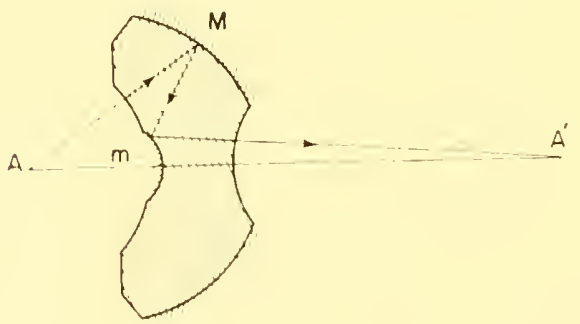

FIG. 9.9. Solid reflecting objective (D. D. Maksutov).

and (5) to form the eyepiece-observed image $A_{2}^{\prime}$. A small field diaphragm is provided at $A_{1}^{\prime}$ to eliminate stray light. The occlusion ratio is 0.30 .

D. D. Maksutov designed and developed the first one-piece systems. (Fig. 9.9.) These objectives, called "solid objectives", were investigated later by C. G. Wayne and R. L. Drew. 
K. P. Norris and M. H. F. Wilkins devised a solid objective, applicable to ultra-violet photomicrography, consisting of 3 quartz elements linked together by optical contact (Fig. 9.10). Two of these elements are aluminized at $M$ and $m$; the occlusion ratio equates 35

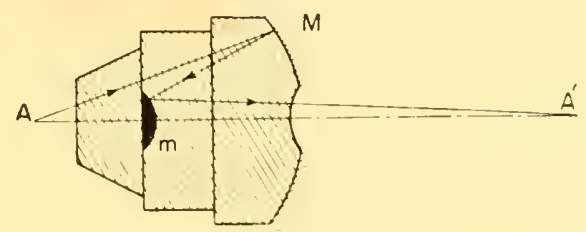

FIG. 9.10. Norris and Wilkins solid reflecting objective.

per cent. The N.A. 0.90 is achieved using water as immersion medium. Bouwers and Blaisse have also developed an ultra-violet solid objective (Fig. 9.11). The element (1) is aluminized in the area $m$, which acts as a convex mirror and is surrounded by a small

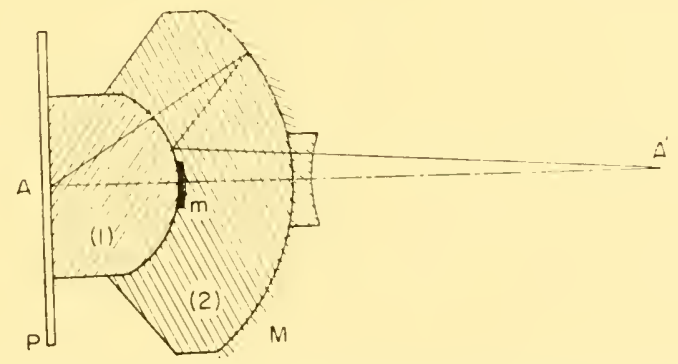

FIG. 9.11. Bouwers and Blaisse solid reflecting objective.

semi-aluminized annular zone. The rays passing through the semialuminized area are reflected on the completely aluminized area $\mathrm{m}$. In this way the occlusion ratio is reduced to $0 \cdot 30$.

Lastly the "ultrafluar" refracting objectives, developed by Zeiss, should be referred to. These objectives are corrected in the 2300 to $7000 \AA$ range and provide very satisfactory imagery. They are eminently suitable for ultra-violet microscopy and available in three types:

$$
\begin{array}{cc}
\text { Ultrafluar } & \times 32 n \sin u=0.40 \\
, & \times 100 n \sin u=0.85 \\
, \quad & \times 100 n \sin u=1.25
\end{array}
$$


These three objectives are of the glycerine-immersion type and complemented by a projection-type eyepiece and an achromatic condenser of N.A. $0 \cdot 8$.

\section{OBSERVING AND RECORDING INFRA-RED} AND ULTRA-VIOLET IMAGERY

Photography in the lower infra-red spectrum is feasible owing to such sensitizers of photographic emulsions as dicyanine, neocyanine, xenocyanine, and so forth. Such sensitizers have been used for dyeing biological specimens. The photographic plate processed by the same sensitizer has utmost sensitivity for the more-absorbed radiations. This process furnishes highly contrasted negatives up to $1 \cdot 3 \mu$ (spectralsensitivity limit of photographic plates). Instead of registering the images photographically, the infra-red image can be observed direct on a fluorescent screen by means of a very simple image converter also called frequency transformers. Figure $9 \cdot 12$ shows schematically an image converter: the semi-transparent photocathode $C$ is set, on

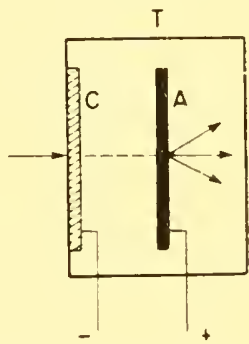

FIG. 9.12. Diagrammatic image converter.

the inner wall of the vacuum-tube $T$. several millimetres away from the anode $A$ which is also a fluorescent screen. A potential difference is originated between $A$ and $C$. Infra-red photons, incoming on $C$, release electrons which are accelerated by the electric field obtaining between $A$ and $C$. The electron pencil of rays appears on the fluorescent screen as a spot. Sharpness is not too good with such apparatus and, in many converters, of which there are many types, the anode is independent of the fluorescent screen. These apparatuses include electrostatic lenses conjoining the photocathode $C$ and the fluorescent screen $A$. Highest spectral sensitivity attains approximately $1.5 \mu$. Some devices ate designed to obtain increased luminance of converted images. 
There are "solid" frequency transformers which might possibly be used in microscopy. They consist of the photoconductor $D$ and the electro-luminescent layer $E$, sandwiched between the two thin transparent conducting layers $C_{1}$ and $C_{2}$ (Fig. 9.13). Both layers $C_{1}$ and $C_{2}$ are deposited on the inner faces of the two glass plates $A$ and $B$. A heavy alternating current is fed through $D$ and $E$ by means of $C_{1}$ and $C_{2}$. As the photons reach $D$ the resistance of the layer drops locally thereby giving rise to the increased potential difference to which

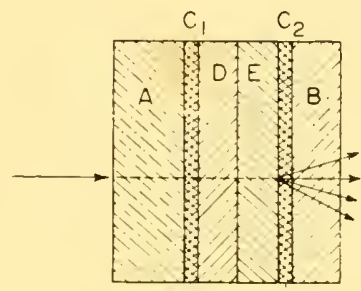

FIG. 9.13. Diagrammatic solid image converter.

the layer $E$ is locally subjected. The layer $E$ radiates visible light opposite the area of the layer $D$ affected by the infra-red radiation. In order to convert an infra-red image into a visible one, television scanning tubes are also suitable. W. Heimann has evolved a tube of this type sensitive up to $3 \cdot 5 \mu$.

In the ultra-violet field, photography is in general use as standard emulsions remain sensitive to wave-lengths up to $0 \cdot 23 \mu$. Figure $9 \cdot 14$ shows the diagram of Burch's microscope for photomicrography in visible and ultra-violet light. The ultra-violet source is at $S_{1}$ (mercury arc without continous spectrum). The lens $L_{1}$ images $S_{1}$ on the slit $F_{1}$ of the monochromator which consists of the objective $L_{3}$, the prism $P$ and the auto-collimator mirror $M_{1}$. Directing suitably the latter, and after reflection on the mirror $M_{2}$, a spectrum is obtained from which a monochromatic portion is isolated by means of the slit $F_{2}$. By means of the lens $L_{5}$ (field lens) and reflection on the mirror $M_{3}$, the slit $F_{2}$ is imaged in the condenser $C$ which is a mirror objective. The condenser $C$ is adjusted so as to image $L_{5}$ on the specimen at $A$ (Köhler illumination), the lens $L_{4}$ imaging $L_{3}$ on $L_{5}$. Uniform illumination in monochromatic light of the specimen is thus obtained. 
The visible-light source $S_{2}$ can be imaged on the slit $F_{2}$ by means of the lens $L_{2}$. Since the microscope is focused in white light, it requires no change in the ultra-violet.

Burch's objective, at $O$, is used minus the eyepiece, thus eliminating its aberrations. With an objective of N.A. 0.65 and a tube length of 345 millimetres, the image projected direct on the photographic plate at $E$ is magnified $\times 110$.

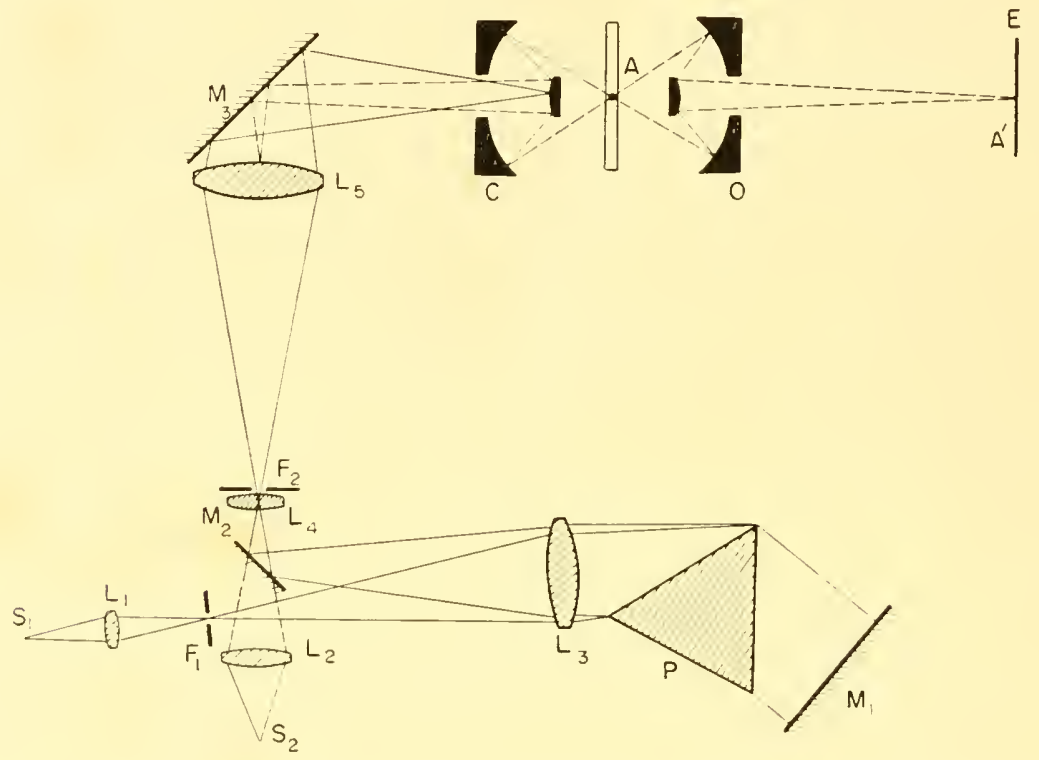

FIG. 9.14. Diagram of the Burch microscope for photomicrography in visible and ultraviolet light.

As in infra-red, transposing an ultra-violet image in visible light is feasible. To convert into colour variations the absorption variations of an object investigated in ultra-violet light, Brumberg devised an ingenious method; he makes three exposures of the object at three different ultra-violet wave-lengths. Next, these three negatives are projected and superimposed on a screen through three differently coloured filters. The colour variations evince the absorption variations. The Polaroid Corporation has devised an apparatus, built on this principle, that promptly provides a coloured image.

Image converters are also used in the ultra-violet. Figure 9.15 shows the Bausch and Lomb ultra-violet microscope to which an R.C.A. converter is fitted. 


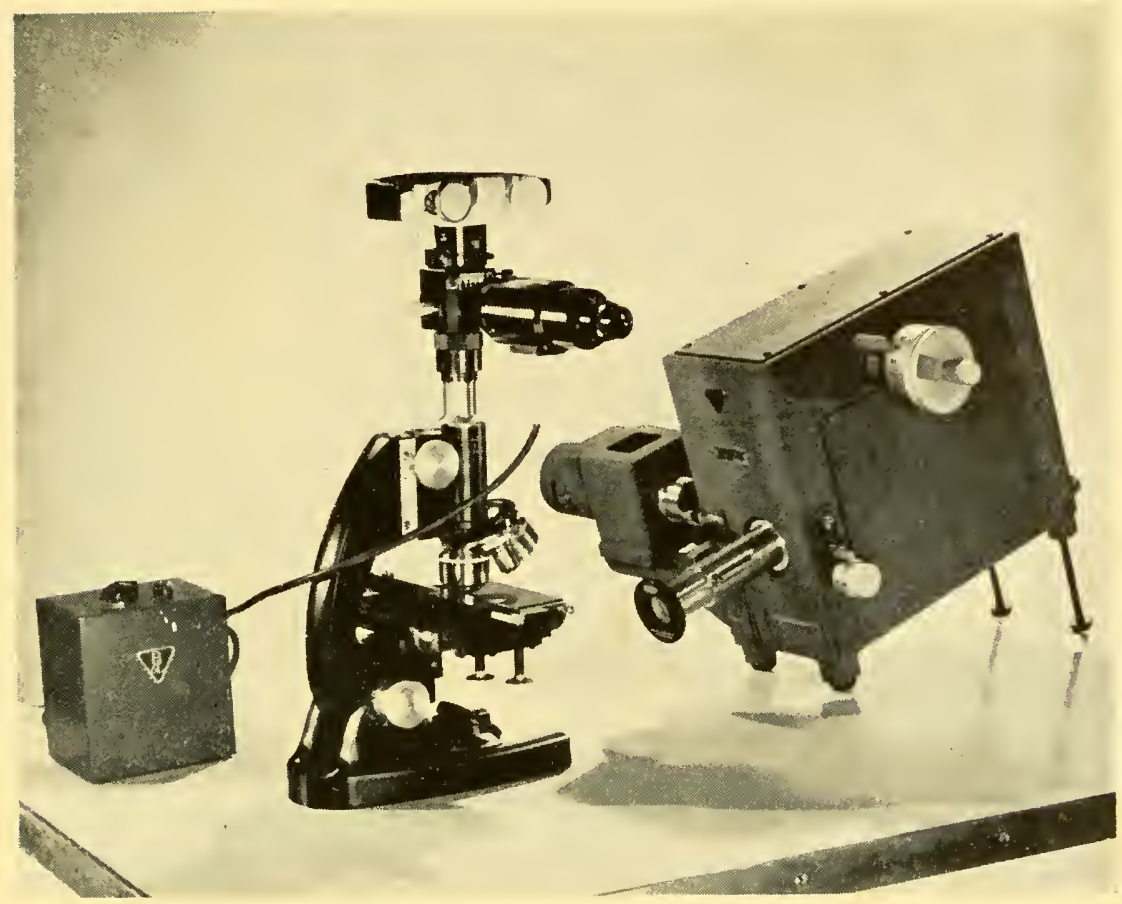

FIG. 9.15. Bausch and Lomb ultra-violet microscope with an R.C.A. image converter.

Reference must be made to receivers of the television camera type. They can function below $2300 \AA$ and, owing to their sensitivity, it is possible to use faint radiations and to irradiate certain objects for some length of time. Television camera tubes have also been used in Brumberg's colour transposition process.

\section{FLUORESCENCE}

Under the effect of ultra-violet radiation, a fluorescent body has the property of emitting visible radiation. This phenomenon enables detection of substances not visible in a standard microscope. In primary fluorescence the substance radiates light which is visible under the effects of ultra-violet radiation only, without any further processing. In secondary fluorescence, the substance is impregnated with a fluorescent solution.

In order to examine the fluorescent image without the objectionable exciting radiation, stop-filters are provided which eliminate the ultra- 
violet beyond the specimen. The fluorescent portions of the specimen show up brightly against a dark ground (Fig. 9.16). A dark-ground condenser (Barer) may also be used to illuminate the specimen thus preventing the incident beam from entering the microscope. Quartz condensers or comprising mirror optical systems may also be used. In the latter type, the surfaces are not to be silvered but, instead, aluminized.

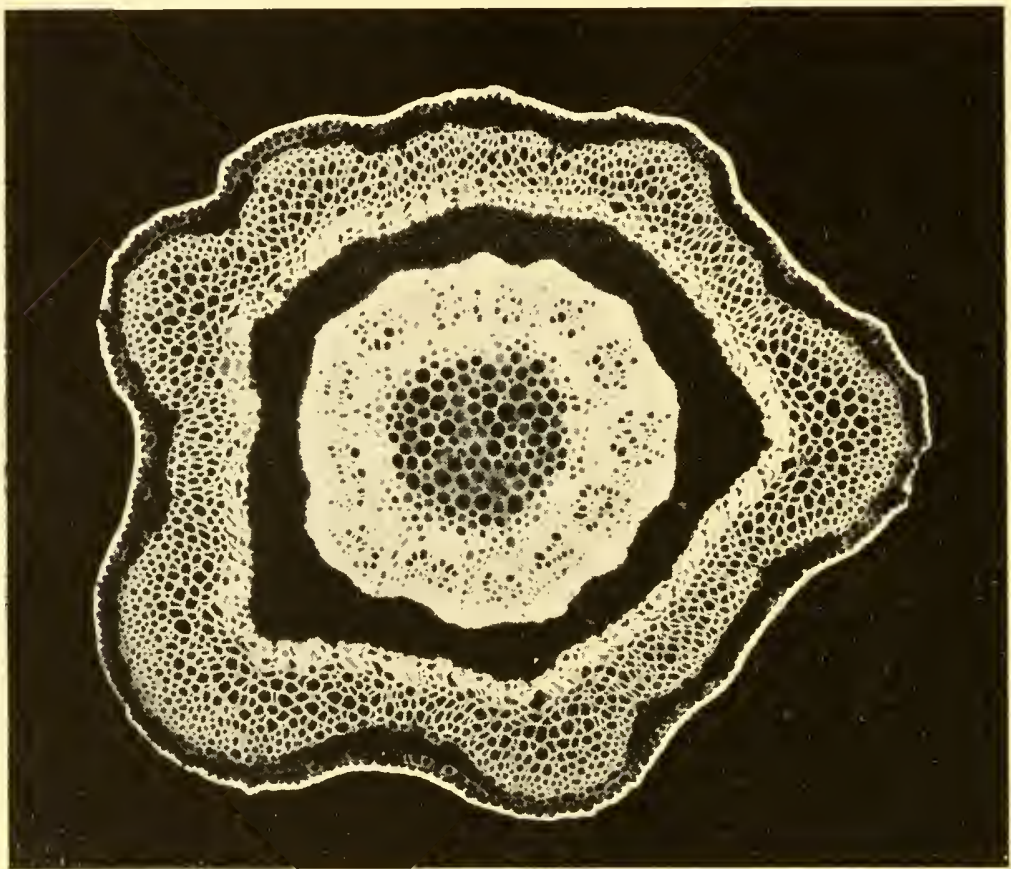

FiG. 9.16. Fluorescence of a cross-section of a stalk (Courtesy of Dr. Gabler-Reichert).

Many biological substances are fluorescent under the effects of wave-lengths below $3650 \AA$ and at which glass is transparent. In such cases a standard condenser is suitable.

When the specimen emits radiation within the visible light spectrum, standard optical systems are used beyond the specimen. The microscope is not to comprise semi-apochromatic or apochromatic objectives since some of their elements are of fluorite whose fluorescence may set in disturbances. Likewise, cedar oil, which is fluorescent, is not suitable as immersion fluid. It can be substituted for non-fluorescent synthetic oils. 
A fluorescent specimen may be examined either in transmitted or reflected light. In the former case, the layout is that of a standard microscope. A filter is set in front of the condenser so that the specimen is irradiated only with the radiation required. Frequently, ultra-violet radiation, extending over a wide wave-length range, can be selected and, then, all that is required is a filter eliminating the visible spectrum. A second filter, set in the microscope proper, lets through the visible fluorescent light but occludes the ultra-violet.

In reflective examinations, various arrangements may be used: those of Lieberkuhn (Fig. 4.17), of Chapman and Alldridge (Fig. 4.18), mirror objectives and so forth. When low magnifying powers are involved and the distance between the objective and the specimen is adequate, illumination can be effected laterally, using an illuminator not integral with the microscope. Lastly, to secure valuable information regarding the details observed, it may be desirable to study the spectral constitution of the fluorescence generated. Such is the object of microspectroscopic fluorescence the fundamentals of which are set forth in Chapter X. 
CHAPTER X

\section{Microspectroscopy and Microspectrophotometry}

\section{FUNDAMENTALS OF MICROSPECTROSCOPY AND MICROSPECTROPHOTOMETRY}

IN microscopy, investigating spectra in the same way as in chemical analysis affords valuable information related to the constitution of small samples. In histochemistry, for instance, it complements the morphological aspect of the problems considered by determining localized substances.

Evolvement of reflecting objectives gave rise to the extension of microspectroscopy. Complete absence of chromatism made them particularly suitable in ultra-violet and infra-red applications as they could be used without altering the focusing used in visible light: obviously a very valuable and practical feature. Generally, microspectrophotography determinations are spectral-absorption determinations. The object, transparent to a lesser or greater degree, need not be destroyed and its absorption is studied versus the wave-length. Conventional spectrum analysis methods apply equally well to microscopy and furnish either qualitative or quantitative analysis.

In qualitative analysis, the spectrum of an area of the specimen is used to seek the presence and define the nature of an unknown substance. Then, the absorption curve of the spectrum is to be plotted in order to determine the position and the structure of absorption bands in a more or less extensive portion of the spectrum.

In quantitative analysis, the purpose sought is to determine the quantity of a substance whose presence is already ascertained. Two procedures may be used:

(a) The object is illuminated in monochromatic light;

(b) The object is illuminated by a complex source and the spectral analysis carried out after the light has passed through the object. 


\section{THE OBJECT IS ILLUMINATED IN MONOCHROMATIC LIGHT}

The arrangement is schematized in Fig. 10.1. The monochromator is set ahead of the microscope and adjusted so that the image of the outgoing-slit $F^{\prime}$ is formed in the condenser $C$ (reflecting objective). The condenser $C$ is adjusted so as to image $l_{3}$ in the specimen $P$.

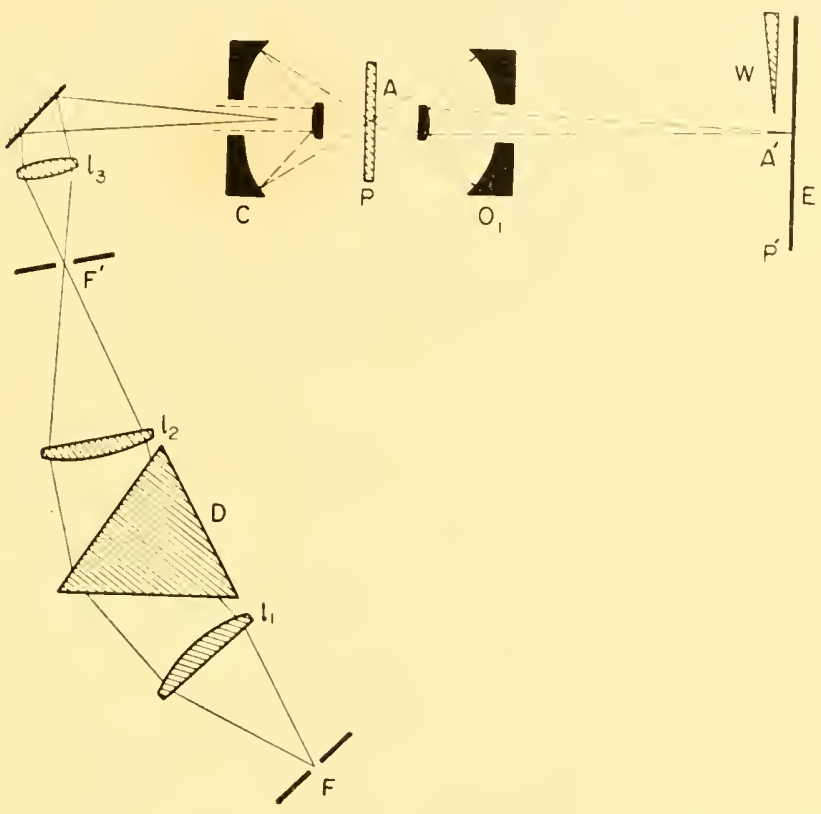

FIG. 10.1. Diagram of a basic system for microspec rophotometry (monochromator before the microscope).

Next the reflecting objective $O_{1}$ of the microscope forms the image $P^{\prime}$ of $P$ in the photographic plate $E$. The layout is like the one shown in Fig. 9.14. Thus is obtained a photograph, in monochromatic light, of the specimen. Once this photograph is microdensitometer-analysed, the absorption obtaining at several points of the specimen at this wave-length, may be compared with a point located in a vacant area of the specimen. Measurements may be carried out with the neutral wedge $W$ against the photographic plate $E$ in a suitably uniform imageless area of the field. Both the wedge and $E$ are illuminated simultaneously by the same wave-length.

The layout, shown in Fig. 10.2, in which the beam illuminating $W$ does not pass through the microscope, may also be used. The semi- 
reflective plate $M_{1}$ splits the incident beam in two parts, one of which passes through the microscope, the other next to it serving to illuminate the neutral wedge $W$.

The imaged wedge on the photographic plate, is developed at the same time and, hence, under the same conditions as the image of the specimen.

The upper portion of Fig. 10.3 shows the aspect of the photographic plate, including the wedge image $W^{\prime}$. The lower portion of the figure

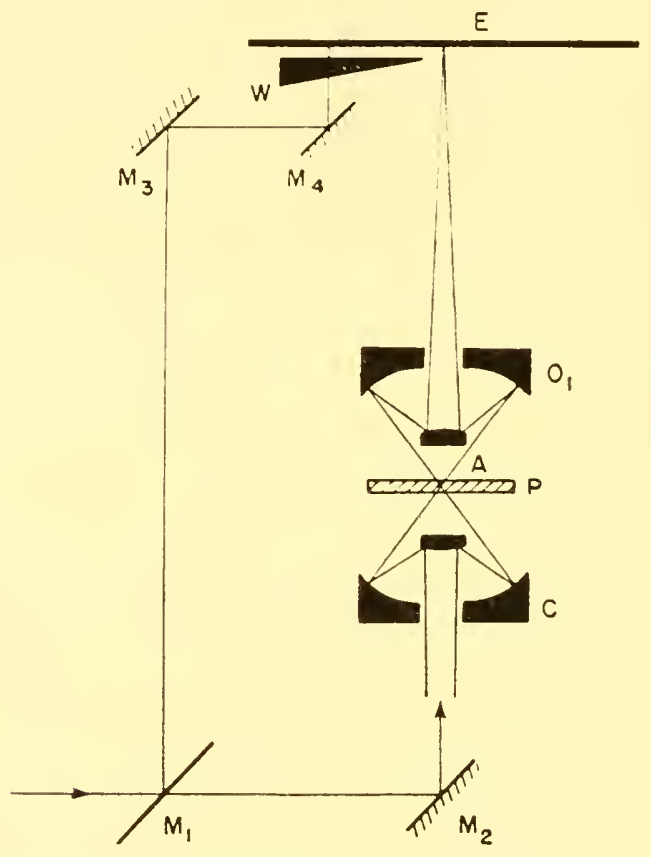

FIG. 10.2. Double beam microspectrophotometric device (P. M. B. Walker).

shows the microdensitometer-analysed negative. This analysis is effected as follows: absorption of the image $W^{\prime}$ is analysed at any random point, the incident and the transmitted intensity being denoted by $I_{0}$ and $I$ respectively. At such point, the transmittance is defined by the ratio $T=I I_{0}$. In actual practice, the optical density $d$, i.e. the common logarithm of the inverse ratio $1 / T$, is frequently used, then:

$$
d=\log \frac{I_{0}}{I}=\log \frac{1}{T} .
$$


The curve (1) shows the densities in the image $A^{\prime}$ of the object along $x x^{\prime}$ while curve (2) shows those in the image $W^{\prime \prime}$ of the neutral wedge along $y y^{\prime}$. The curve in Fig. 10.4 is derived by plotting the photographic densities $d_{p}$ as ordinates (densities measured along.$y^{\prime}$ )

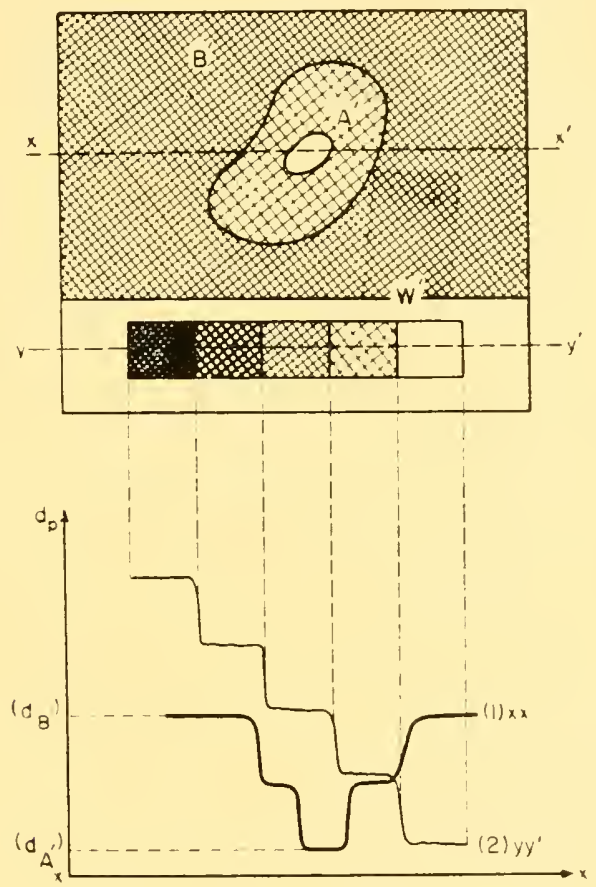

FiG. 10.3. Analysis of the negative.

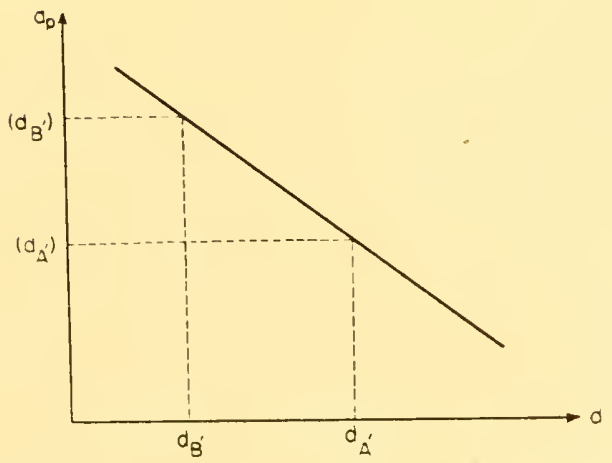

FIG. 10.4. Abscissae: actual densities $(d)$ of the neutral wedge. Ordinates: densities $d_{p}$ measured along $y y^{\prime}$ (Fig. 10.3). 
and the actual densities $(d)$ (of the neutral wedge) as abscissae. The area, i.e. $B^{\prime}$ is a vacant area and is going to be investigated by comparing it with the absorption of the detail being examined. The curve(1) in Fig. 10.3 denotes the photographic density $\left(d_{B^{\prime}}\right)$ for $B^{\prime}$. Re-plotting this value on the curve in Fig. 10.4 denotes the actual density $d_{B^{\prime}}$ at $B^{\prime}$. Likewise the photographic density $\left(d_{A^{\prime}}\right)$ of the detail $A$ is obtained (Fig. 10.3), to which the actual density $d_{A^{\prime}}$ is correlated (Fig. 10.4). In relation to $B^{\prime}$ the density $d_{A^{\prime} B^{\prime}}$ of the detail $A^{\prime}$ is:

According to $(10.1)$

$$
d_{A^{\prime} B^{\prime}}=d_{A^{\prime}}-d_{B^{\prime}} .
$$

$$
d_{A^{\prime}}=\log \frac{1}{T_{A^{\prime}}} \quad d_{B^{\prime}}=\log \frac{1}{T_{B^{\prime}}}
$$

may be written and, provided $T_{A^{\prime} B^{\prime}}$ be the transmittance of $A^{\prime}$ in relation to $B^{\prime}$, then:

$$
T_{A^{\prime} B^{\prime}}=\frac{T_{A^{\prime}}}{T_{B^{\prime}}} \quad d_{A^{\prime} B^{\prime}}=\log \frac{T_{B^{\prime}}}{T_{A^{\prime}}}=\log \frac{1}{T_{A^{\prime} B^{\prime}}} .
$$

The cquations applicable to microspectrophotometric determinations are (10.2) and (10.3). The foregoing measurements evince $T_{A^{\prime} B^{\prime}}$ and $d_{A^{\prime} B^{\prime}}$ for a single wave-length. Measurements are to be resumed for the other wave-lengths of the spectrum and the curve, showing the $T_{A^{\prime} B^{\prime}}$ or $d_{A^{\prime} B^{\prime}}$ variations versus the wave-length, are to be plotted. When the specimen and the object itself are considered, spectroscopists substitute the word "density" for "absorbance": the word "density" is solely applied to measurements carried out with the photographic plate. The latter may be replaced by a photoelectric cell (Fig. 10.5). The source $S$, fed by the stabilized powersupply unit $V_{1}$, illuminates the monochromator whose outlet slit $F$ acts as source of monochromatic light. The microscope consists of the condenser $C$ and the objective $O_{1}$, the latter imaging the specimen $P$ on the screen $E$. A small aperture, made in the screen $E$, isolates the imaged portion to be photo-analysed. The photo-multiplicr $R$, fed by the stabilized power-supply unit $V_{2}$, is set at the back of $T$, the lens $l_{2}$ imaging $O_{1}$ on $R$. The image $A^{\prime}$ of the investigated detail $A$ overspreads the aperture $T$ and the measuring instrument shows a deflection $D_{A^{\prime}}$. Shifting the specimen evinces a deflection $D_{B^{\prime}}$ for the area $B^{\prime}$. The transmittance of $A^{\prime}$ in relation to $B^{\prime}$ is $T_{A^{\prime} B^{\prime}}=D_{A^{\prime}} / D_{B^{\prime}}$, whence the density $d_{A^{\prime} B^{\prime}}$. 
When photographic layouts are involved, a double beam arrangement may be used (Fig. 10.6). The light from the monochromator (not shown in Fig. 10.6) may follow alternatively two different paths: the normal path through the microscope and the comparison path, ending direct on to the photomultiplier $R$. The mirror $m_{1}$ moves to and fro, thus alternating the beams corresponding with both paths.

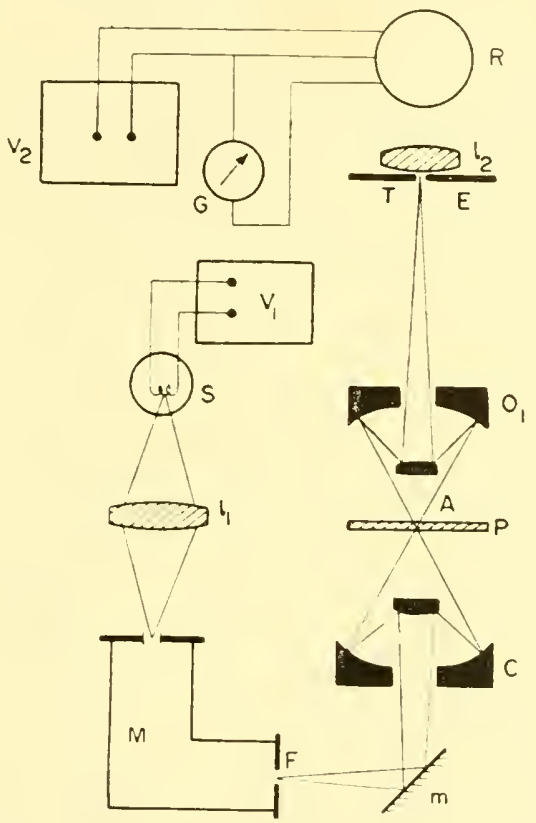

FIG. 10.5. Diagram of a basic system for microspectrophotometry with photo-cell.

The objective $O_{1}$ images the specimen $P$ on the diaphragm $T_{1}$, set close to the lens $l_{1}$. The lens $l_{1}$ images $O_{1}$ on the photomultiplier. The lens $l_{2}$, athwart the comparison beam, ensures uniform illumination of the photomultiplier $R$. The adjustable diaphragm $T_{2}$ is set against $l_{2}$ while the continuous adjustment of the intensity of the reference beam is provided by the neutral wedge $W$. The photomultiplier-supplied signal is amplified in the amplifier $A M$ and eventually fed to the oscillograph $U$.

The measurement consists in equalizing the signals generated by the two beams, this being accomplished by merely shifting $W$ whose positions are shown by a graduated scale. Since the image $A^{\prime}$ of the detail $A$ is formed on $T_{1}$, the signals are equalized when the neutral wedge $W$ is in the position $W_{A^{\prime}}$. 
Setting on $T_{1}$ the image $B^{\prime}$ of the vacant area $B$ of the specimen. the reading is $W_{B^{\prime}}$. The transmission factor $T_{A^{\prime} B^{\prime}}$ of $A^{\prime}$ in relation to $B^{\prime}$ is given by $T_{A^{\prime} B^{\prime}}=W_{A^{\prime}} / W_{B^{\prime}}$ whence the density $d_{A^{\prime} B^{\prime}}$ of the detail $A^{\prime}$.

In the Thorell arrangement, the vibrating mirror $m_{3}$ is set between the objective $O_{1}$ and the small aperture $T_{1}$ (Fig. 10.7), the latter being

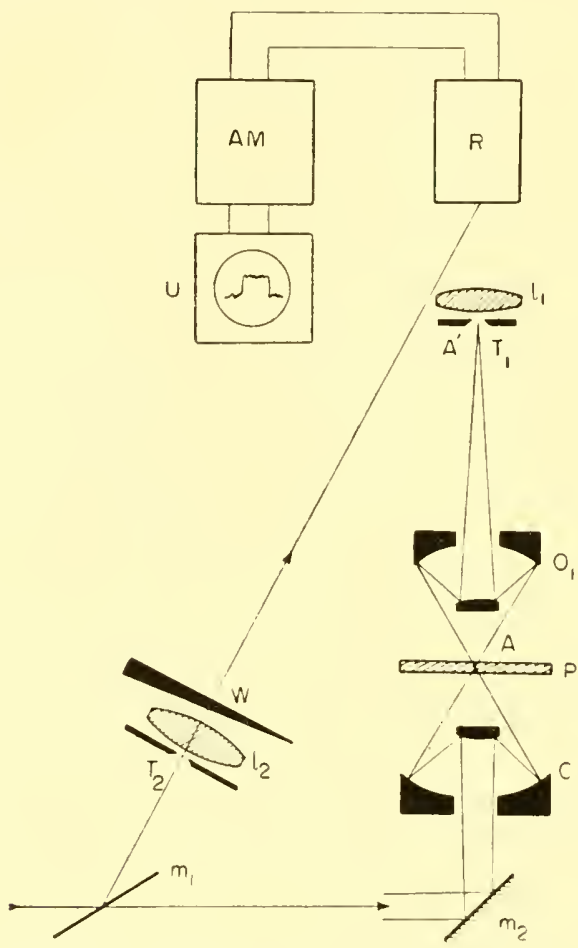

FIG. 10.6. Double beam arrangement with photo-cell (P. M. B. Walker).

alternately spread over by the imaged detail being studied and the imaged vacant area of the specimen. It follows that the reference beam, passing through $W$, is dispensed with (Fig. 10.6).

T. Caspersson, who blazed the trail of ultra-violet microspectrography, has devised an extensive equipment for surveying quantitative cytochemistry applications (shown in simplified form in Fig. 10.8).

The objective $O_{1}$ of the microscope images the specimen on a diaphragm provided with the small aperture $T_{1}$ behind which is the cell $R_{1}$. The comparison beam, extraneous to the microscope, enters the second cell $R_{2}$. The current $I_{1}$, generated by $R_{1}$, is automatically 
compared with the current $I_{2}$, generated by $R_{2}$, used as reference. This comparison takes place in the electrical apparatus $R C$. The absorption changes within the object, at a given wave-length, can thus be determined. The shift of the specimen is linked to the motion of the paper strip of the recorder $D$ which plots the curve $I_{1} / I_{2}$ with respect to the position of the point investigated in the object. Motions of both specimen and recorder are provided by the motor MO. The gear box $E G$ is connected to two selsyn $S l_{1}$ and $S l_{2}$, thus enabling
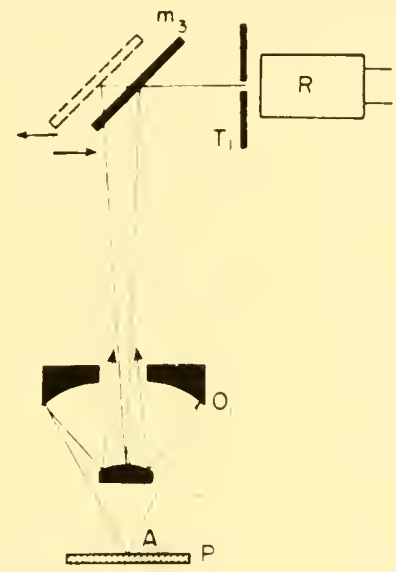

FIG. 10.7. Thorell arrangement.

to adjust ad libitum the ratio of their speeds of rotation. The selsyn $\mathrm{Sl}_{2}$ drives the selsyn $\mathrm{Sl}_{3}$ which itself controls the paper-strip motion of the recorder $D$. The selsyn $S l_{1}$ also drives the selsyn $S l_{4}$ which, through the device $S P$ (described later), controls the motion of the specimen. Motions of both specimen and recorder therefore take place according to a definite ratio. In these measurements, the wavelength of the light supplied by the monochromator, is constant. The wave-length of the light used can be modified, at will, by connecting the selsyn $S l_{1}$ to the selsyn $S l_{5}$. The recorder may also be substituted for the oscillograph $U$. The specimen is shifted according to a definite direction by means of a highly sensitive device ensuring a sensitivity attaining $0 \cdot 1 \mu$. The chamber $B_{1}$ contains some mercury (Fig. 10.9) and is connected through the pipe $t$ to the element $B_{2}$, the two latter being filled with mercury. The diaphragm of $B_{2}$ is connected to the specimen and controls its motion. The unit comprising $m, B_{2}, t, B_{1}$. and the screw $V$, forming the device $S P$, is shown 
in Fig. 10.8. The object of Fig. 10.9 is to show the identical devices $S P$ and $R C$ in greater detail and their different locations. As mentioned previously, the motor $M O$ actuates the specimen through the selsyn

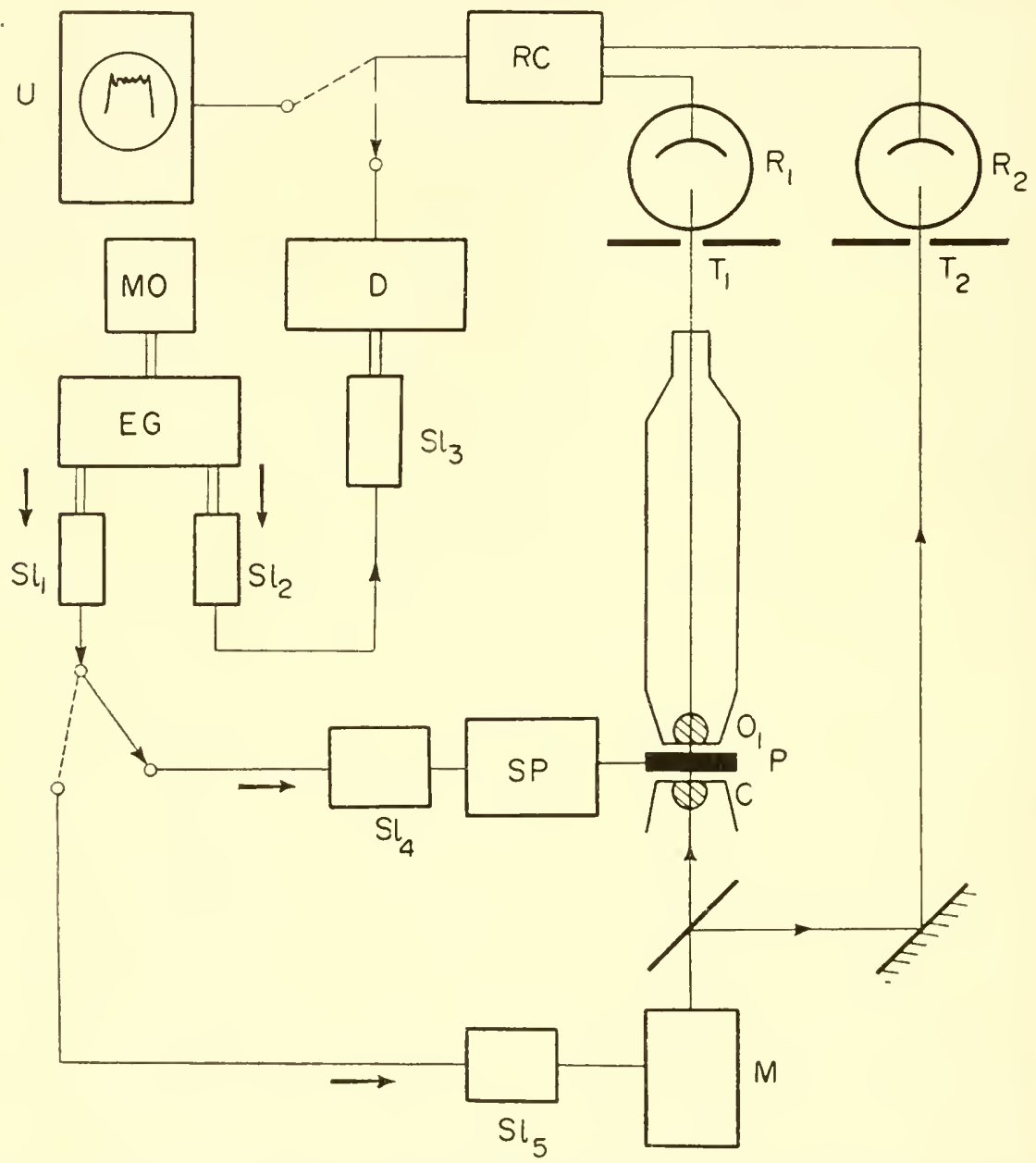

FIG. 10.8. Diagram of the Caspersson microspectrophotometric equipment for surveying quantitative cytochemistry applications.

$S l_{1}$ and $S l_{4}$. The latter (Fig. 10.9) actuates a screw that penetrates into a chamber and, by compression, alters the mercury level in $B_{1}$. The pressure causes the mercury to rise into $B_{2}$ thus shifting the diaphragm actuating the specimen $P$. The motor $M O$ drives also the recorder $D$ whose pen $I$, driven by the device $R C$ (itself driven by the 
motor $M O^{\prime}$ ), plots the curve showing absorption in terms of the position within the specimen.

Once the curve is plotted according to a specific path within the object, the specimen should be shifted slightly in a perpendicular direction.

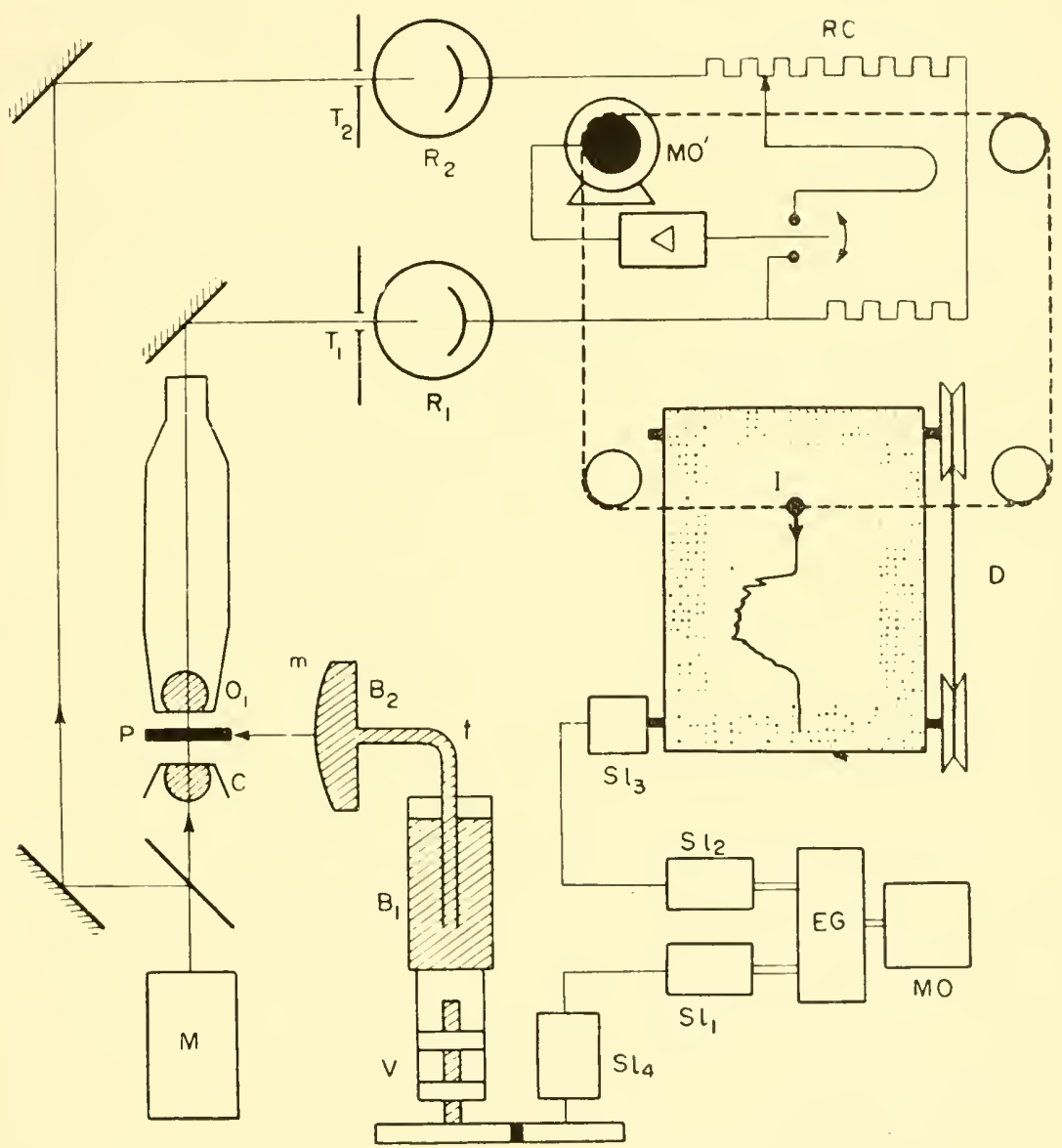

Fig. 10.9. Motor $M O$ actuates the specimen $P$ through the selsyn $S I_{1}$ and $S l_{1}$, the screw $V$ and the device $B_{1} t B_{2} m$. Motor $M O$ drives also the recorder $D$ whose pen $I$ plots the absorption curve of the specimen.

This shift is required in order to plot another absorption curve according to a line parallel with the former. Such shift is provided by a plate, set after the objective $O_{1}$, and whose tilt changes bring about a slight shift of the imaged specimen. The arrangement is 
devised so that such shift be directed at right angles to the shift originated by the diaphragm $m$. Thus is derived the absorption distribution over the whole area of the object.

Note 1. In devices similar to the one shown in Fig. 10.5, measurements only deal with a small portion of the image to which is correlated an even smaller portion of the field object. Then the outlet slit $F$ of the monochromator may be imaged on to the specimen. Even with a narrow slit providing fully-monochromatic light, the width of the illuminated field is always adequate. In layouts similar to the one shown in Fig. 10.1, the whole field object at $E$ is included in the photographic plate. If the slit of the monochromator is imaged on the specimen, the slit is to have adequate width, covering the field but this may give rise to wave-length changes throughout the image. Conversely, the whole field is illuminated without any such change if the slit is imaged in the condenser, duly adjusted to obtain the image of $I_{2}$ (Fig. 10.1) on the specimen. Reducing the slit increases monochromatism without lessening the width of the illuminated field.

Note 2. In layouts similar to the one shown in Fig. 10.1, the specimen is illuminated throughout. The photo-analysed area receives some stray light from all the illuminated points of the specimen. Such light is due to the optical elements of the microscope and diffraction-originated effects. Every image element is a diffraction disk spreading the light. Hence, luminous elements close to the studied detail may convey light there owing to their diffraction rings. Such effect can be very objectionable if the investigated object is close to a much brighter detail. These drawbacks can be avoided by illuminating only a very small area, instead of the whole specimen.

Discounting design problems, setting effectively a diaphragm against the object solves the difficulty. Since such an arrangement is but seldom feasible and only when low magnification is involved, the following method is to be preferred and applies to all cases.

The light, originated from the monochromator, passes through the microscope in reverse. A diaphragm, provided with a small aperture, is set in the plane of the spectrum furnished by the monochromator. This aperture is imaged on the specimen by the microscope objective. The emerging light impinges against the condenser and proceeds to the photoelectric cell. Figure 10.10 shows diagrammatically the layout of the device. The diaphragm $T$ with its small aperture, is in the position of the outlet slit of the monochromator. 
After being reflected in the $45^{\circ}$-slanted semi-transparent mirror $G$, the light enters the objective $O_{1}$ which images, on the investigated specimen $P$, the hole $T$ reduced by the magnification of the objective $O_{1}$ used. This is the image that serves to illuminate the detail investigated. Since any diameter can be given to it, only the detail

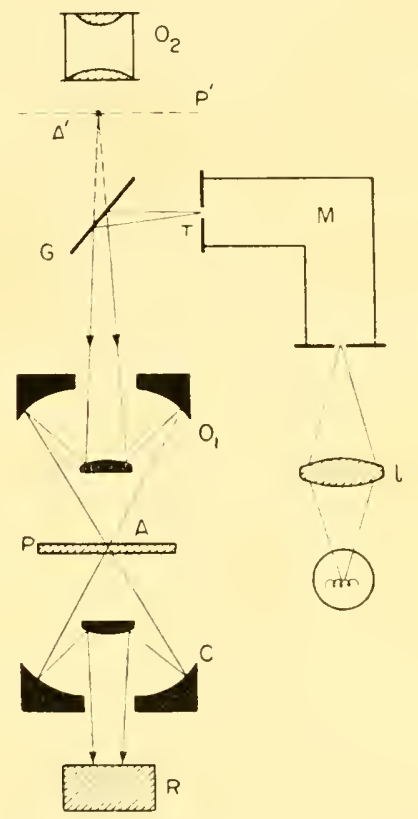

FIG. 10.10. Mcrospectrophotometric arrangement in which the light originated from the monochromator passes through the microscope in reverse.

investigated need be illuminated. The condenser $C$ sends the light having passed the detail investigated to the cell $R$. By means of a retractable mirror, not shown in Fig. 10.10, the specimen can be illuminated and observed in the customary way. If the image plane $P^{\prime}$. observed through the eyepiece $O_{2}$, is symmetrical with the diaphragm $T$ in relation to the semi-reflecting face of the mirror $G$, the specimen and the imaged aperture $T$ in the latter are seen by diffusion. This enables one to ascertain that the detail observed is the one to be photo-analysed. Proper alignment can be checked by means of a reticle, at $A^{\prime}$. Luminosity of the device can be increased by leaving the slit of the monochromator at $T$ and inserting a cylindrical lens between $G$ and $T$. In this way, all the light from the monochromator 
is focused on a virtually pin-point image on to the specimen. This method is suitable for such arrangements as those shown, e.g. in Figs. 10.5 and 10.6 .

\section{THE OBJECT IS ILLUMINATED IN WHITE LIGHT}

Figure 10.11 shows the diagram of this arrangement. The specimen $P$ is illuminated by a source of light (not shown in Fig. 10.11) furnishing a continuous spectrum. The objective $O_{1}$ projects an enlarged image of $P$ on the slit $F$ of the spectrograph. The spectrum is collected

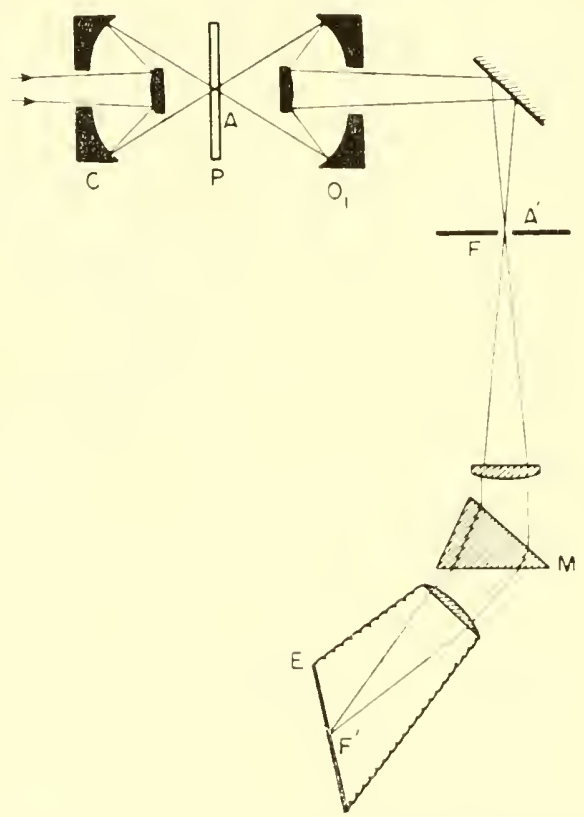

FIG. 10.11. Diagram of a basic system for microspectrophotometry in which the object is illuminated in white light.

on the photographic plate at $E$. The investigated detail $A^{\prime}$ is conveniently set so that it overspreads only a portion of the slit $F$, e.g. the lower part. The upper part is overspread by a vacant area of the specimen serving as reference and, on said part, a rotatable and adjustable sector is set which furnishes on the plate $E$ a series of spectra whose black levels provide a photometric scale for all wavelengths. As in the methods described in $\$ 2$, the photographic plate may be substituted for physical receivers. 
In the method just described the specimen is illuminated simultaneously by all the radiations of the spectrum, this being inconvenient from the stray-light standpoint. Nevertheless, the method is suitable in some cases, e.g. when investigating a transient phenomenon. Yet, whenever feasible, the methods in which the object is illuminated in monochromatic light are to be preferred.

\section{MICROSPECTROPHOTOMETRIC MEASUREMENTS}

As in conventional spectrophotometry, quantity determination and identification of a substance can be made by microspectrophotometry.

In one instance, the spectrum recorded by one of the previous methods is compared with the spectra of known substances, spectra derived from the selfsame instrument. When the detail investigated exhibit a typical spzctrum, this is readily carried out.

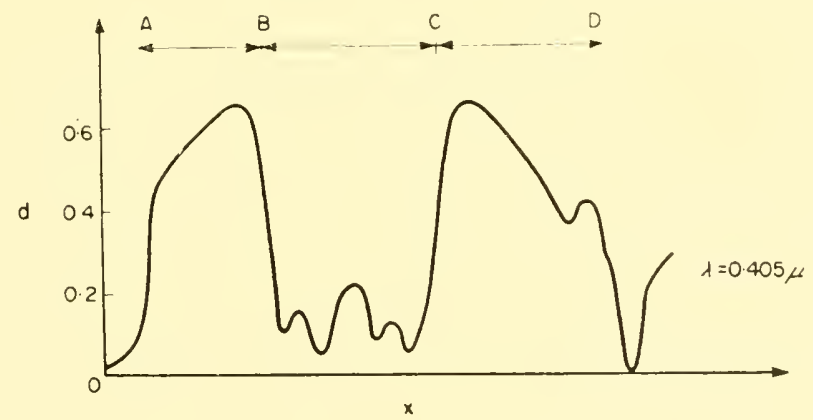

FIG. 10.12. Absorption curve of an erythrocyte from a newt (After Barer, Holiday and Jopes).

Figure 10.12 shows, according to Barer, Holiday and Jopes, the absorption (absorbance) curve of an erythrocyte (blood red cell comprising a nucleus) from a newt, showing this erythrocyte schematically (Fig. 10.13) the absorbance measurement is effected, for instance, along the line $o x$ at a specific wave-length $(\lambda=0.405 \mu)$. The areas $A B$ and $C D$, in Figs. 10.12 and 10.13, correlate the cytoplasm; and the area $B C$ the nucleus, respectively. A family of curves, similar to the one shown in Fig. 10.12, can be plotted for all the spectrum wavelengths. Absorption variation versus the wave-length, taking place at specific points of the specimen, can also be determined. In both cases the curves in Fig. 10.14 show the outcome. The curve $N$ shows the 
absorbance variations of the nucleus versus the wave-length and the curve $C y$ those of the cytoplasm variations. Cytoplasm absorption at approximately $0.415 \mu$ correlates the characteristic spectral absorption region of haemoglobin (Soret) which is known by macrospectrophotometric means. Absorption of the nucleus at approximately $0.260 \mu$ is due to nucleic acid.

In quantitative microspectrophotometric determinations the purpose is to define the concentration of a given substance in the investigated

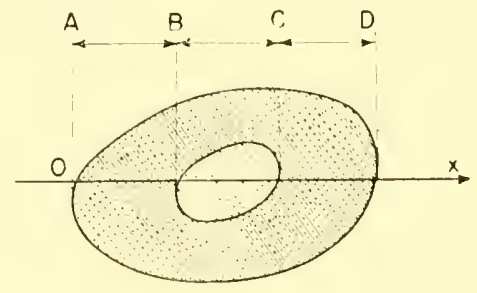

F.G. 10.13. The absorption measurement is effected for instance along the line $O X$ in the image of the cell.

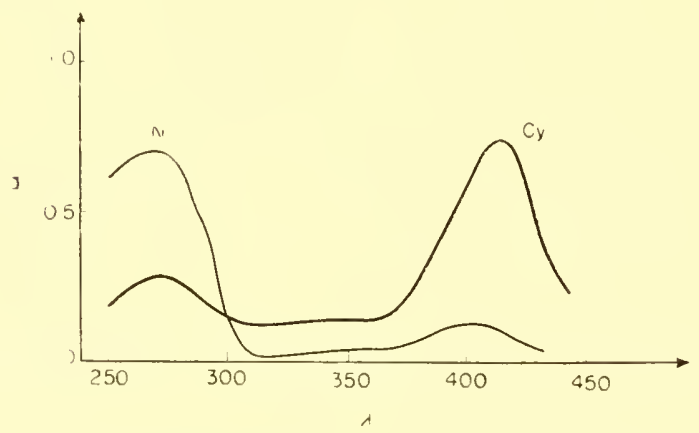

FIG. 10.14. Absorption variation versus the wave-length (after Barer, Holiday and Jopes): Curve $\mathrm{N}$, absorbance variations of the nucleus; Curve $C y$, absorbance variations of the cytoplasm.

object. Determinations may be either absolute or relative when comparing the concentration of the substance in two different elements of the specimen.

Let us assume that microspectrophotometric measurements evince, in the investigated object, the absorbance $d_{\lambda}$ at the wave-length $\lambda$. Let $/$ be the thickness of the object (in $\mathrm{cm}$ ), $C$ the concentration of the substance within the object (in grammes per litre) and $K$ the absorptivity (extinction coefficient) of the substance at a given wave- 
length. Provided Beer's law be applicable, then:

$$
d_{\lambda}=K / C \text {. }
$$

Measuring the concentration $C$ requires, therefore, measuring the thickness $I$ of the object. Such measurement is not always easy to carry out and it is more convenient to substitute it for an area measurement thus leading to the determination of the mass of the substance in the object, instead of concentration.

Let $M$ be the mass of the substance in the object and $s$ its surface, then:

$$
C=\frac{M}{s l}
$$

whence

$$
M=\frac{s d_{\lambda}}{K} .
$$

Provided $K$, at the wave-length considered, be known and having measured $d_{j}$ and the area $s$ of the object, the mass $M$ of the substance in the object is educed. Equation (10.6) shows that, when small quantities of substance are to be measured, the areas must be small too. In an area of $1 \mu^{2}$, approximately $5 \times 10^{-14} \mathrm{gr}$ of nucleic acid may be detected $(K=20$ at $2600 \AA$ ). These numerical values correspond to $d_{\lambda}=0 \cdot 1$.

In this way, Thorell measured the quantity of haemoglobin in a single living cell. The curve in Fig. 10.15 shows the changes in absorption $1-I / I_{0}\left(I_{0}=\right.$ incident intensity, $I=$ intensity transmitted after passing through the object) along the line $0 x$. Absorption at any point of the object can be determined by scanning. Once the shape and dimensions of the cell are known, the hacmoglobin concentration at any point of the object can then be calculated. The aggregate quantity $M$ of haemoglobin is eventually computed by integrating the absorption curve over the whole area $S$ of the cell. According to equation (10.6), then:

$$
M=\iint_{s}^{d}{ }_{K} d x d y .
$$

Equation (10.6) also shows that there is no need to determine $K$ if all that is required is to compare the masses $M_{1}$ and $M_{2}$ of the same substance in two different objects. This is the problem which arises 
when it is required to compare the relative contents of the selfsame substance in two different histological structures. Let $s_{1}$ be the area of the object including the mass $M_{1}$ of the substance and $s_{2}$ the area of the other object including the mass $M_{2}$ of the selfsame substance.

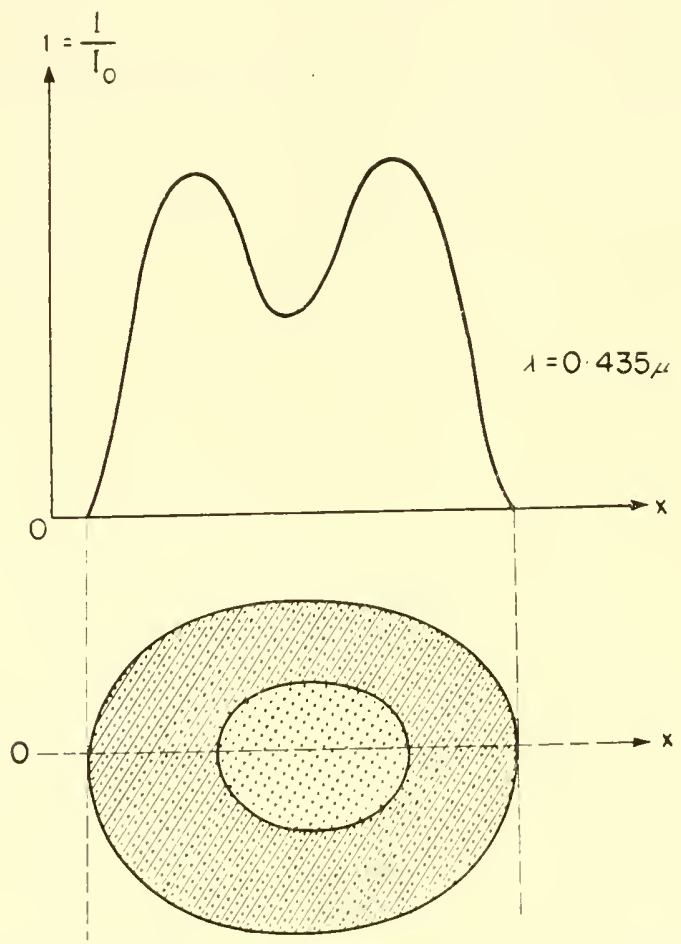

FIG. 10.15. Quantity measurement of haemoglobin in a single cell (Thorell).

Microspectrophotometric measurements evince absorbance $\left(d_{\lambda}\right)_{1}$ for one of the objects and $\left(d_{2}\right)_{2}$ for the other. Equation (10.6) allows one to write:

$$
\frac{M_{1}}{M_{2}}=\frac{\left(d_{\lambda}\right)_{1}}{\left(d_{\lambda}\right)_{2}} \cdot \frac{s_{1}}{s_{2}}
$$

\section{CAUSES OF ERRORS IN MICROSPECTROPHOTOMETRY}

The first cause of error is ascribable to stray light, present in all monochromators. The outlet slit lets through, not only the monochromatic radiation required but also a continuous background, consisting of all the spectrum radiations. This continuous background 
conduces to under-estimate absorbance of the investigated object because the background brings about a drop in contrast of the object in relation to the field surrounding it. Such background is best minimized by setting a second monochromator next to the first (double monochromator). The role of the stray light in the microscope is prominent, too. The undesirable haze it generates on the image may distort measurements. To minimize stray light to the utmost, the illuminated area of the specimen should be as small as possible so that the other areas, not involved in the measurement, do not add their own quota of stray light. This is why an inverted microscope is particularly suitable.

Owing to the condenser aperture, beams at various angles may pass through the object. Then, absorption is not the same as when parallel beams are involved. Blout, and later Walker, have investigated the measurement corrections required. If the N.A. of the condenser is 1 , measuring an object (living cell) of index 1.37, conduces to a 12 per cent over-measurement (absorbance measured close to unity). If the object has an index of 1.53 (fixed cell), the error does not exceed 4 per cent and, when the N.A. of the objective is 0.6 , the two errors drop to 5 per cent and 1 per cent, respectively. Another cause of error may stem from the lack of uniformity of the object itself. Let us now consider an object of constant thickness and another one whose thickness varies. The projected areas of both objects on the observation plane are identical. Assuming that the images of both objects can enter the cell through the aperture $T$ (Figs. 10.5 and 10.6), it is found that measurements diverge and an error regarding the quantity of substance is made. To minimize such an error (caused by the non-uniformity of the object) the diameter of the aperture should be reduced to a bare minimum so that the small portion of the image passing through $T_{1}$ can be considered as uniform. Due consideration must be given to aberrations of the objective. For instance, objectives comprising two spherical mirrors, of N. A. exceeding 0.50 (spherical aberration corrected but field reduced by the coma), can be used in microspectroscopy but the objects investigated must, of course, be kept within the small field where coma is admissible ( $10 \mu$ for an aperture of 0.65 at $2500 \AA$ ). Outside this field the coma causes a considerable drop in the maximal central intensity thus reinforcing the diffraction rings. Since the lossof maximal-centre energy is proportional to the square of the distance to the centre of the field (D. S. Grey), coma effects increase rapidly beyond the admissible field, to the extent 
that connecting the object and image intensities is no longer feasible. thus preventing microspectrophotometric measurements altogether. Besides all the foregoing causes of error, diffraction laws set a limit to the size of the smallest objects observable in microspectrophotometry. When the dimensions of the object approximate the wavelength, a non-negligible portion of the object-diffracted light is lost and cannot enter even the most powerful objectives. Therefore, an absorbance error is made. On the other hand, distribution of the diffracted light varies as the wave-length. In relation to the incident light, the spectral distribution of the light diffracted and focused on the image is altered. But such modification is not due to absorption of the object, thus giving rise to another error. Furthermore, when reflecting objectives are involved, the occluding effects of the small mirror on the diffraction disk are to be considered. The reinforced diffraction rings further reduces the dimensions of an object below which microspectrophotometric measurements can no longer be made. It seems that the smallest dimensions of investigated objects should not fall below 4 to $5 \%$ approx. At a $2500 \AA$ wave-length, details approximating one micron can be measured whereas, in the infra-red. close to $\lambda=20 \mu$ measurements should be restricted to objects whose dimensions approximate $80 \mu$.

\section{PHOTOMETRIC EYEPIECES}

In some cases, simple visual instruments may prove to be serviceable. They usually consist of an eye-lens enabling comparison of the luminance of an imaged area with that of a comparison area of variable luminance. In Leitz's microscope photometer (Berek) the same source illuminates the specimen and the comparison area (Fig. 10.16). There is no need, therefore, to stabilize the light source. The light-beam originated by the source of light (not shown in Fig. 10.16), is split in two by the semi-reflecting cube $M_{1}$. One of these portions travels along the path (1) and serves to illuminate the microscope normally. The other portion travels along the path (2) outside the microscope and serves as comparison beam. The two beams meet again in the cube $M_{4}$. One half of the cemented face of $M_{4}$ is opaque and reflective: it is illuminated by the comparison beam (2). The other half lets through the microscope-originated light. The microscope eyepicce $O_{2}$ is so designed as to allow focusing simultaneously the investigated image and the line bounding the comparison area. 
Appearance of the field is shown in Fig. 10.17. The image $A^{\prime}$. whose luminance is being measured, is compared with the comparison area $A_{c}^{\prime}$, whose luminance is adjustable ad libitum. Such adjustment may be made in several ways. In Fig. 10.16, luminance variation of

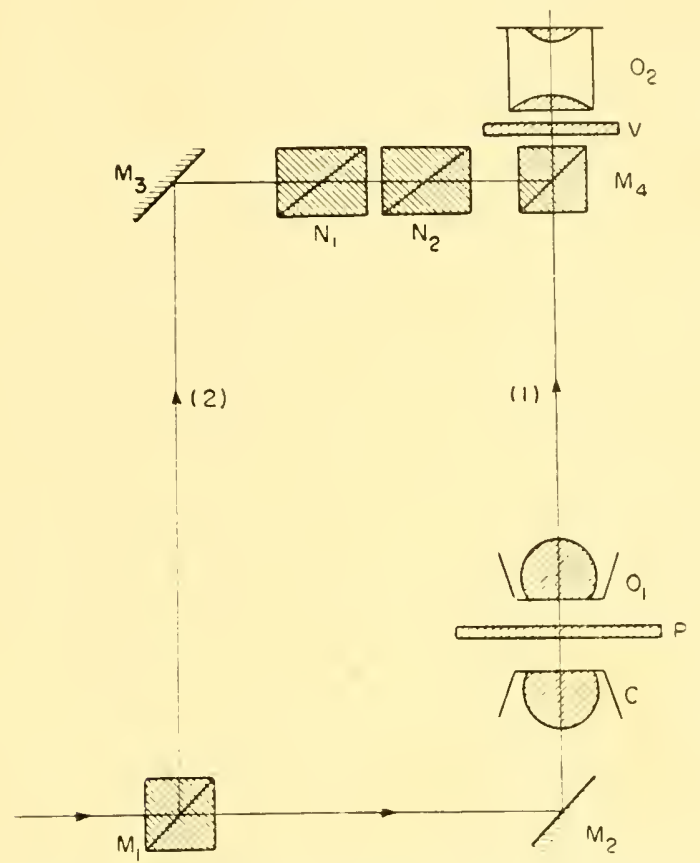

FiG. 10.16. Leitz's microscope-photometer (Berek).

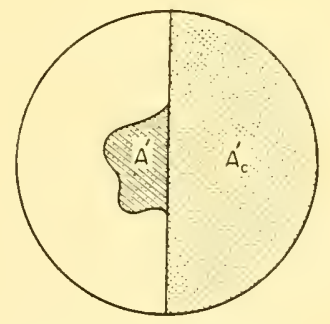

FIG. 10.17. Field aspect.

the comparison area is obtained by means of the two polarizers $N_{1}$ and $N_{2}$. Rotating $N_{2}$, whose orientation is shown by a graduated circle adjusts the luminance of the comparison area until luminances of $A^{\prime}$ and $A_{c}^{\prime}$ are equalized. The filter $V$ enables one to provide the 
same result in monochromatic light. The photometer assembly proper is adaptable to a standard microscope. There is a similar type of instrument designed for photometric work with opaque objects.

The Cooke, Troughton and Simms photometer eyepiece may be used, either with a separate light source, or a single source as in Berek's

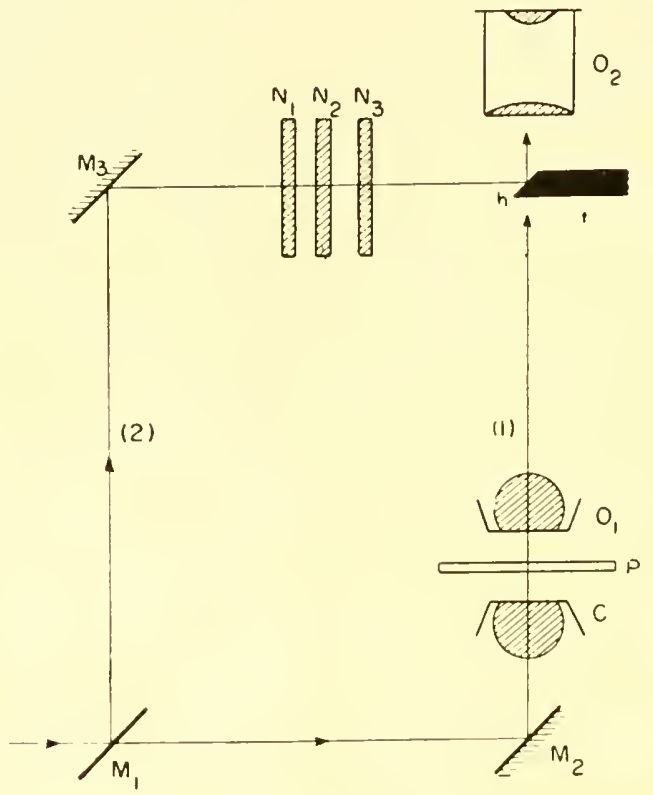

FIG. 10.18. The Cooke, Troughton and Simms photometer eyepiece.

device. The comparison surface consists of the small metallized surface $h$, located at the tip of the glass rod $t$ and cut at $45^{\circ}$. The device for adjusting the luminance of the comparison surface consists of the three polarizers (polaroid) $N_{1}, N_{2}, N_{3}$.

The polaroid elements $N_{1}$ and $N_{3}$ are fixed and their axes parallel. The polaroid $N_{2}$ is rotatable and its orientation shown by a graduated circle. Under such conditions, the transmitted intensity is subjected to a $\sin ^{4} \theta\left(\theta=\right.$ angle of rotation) instead of the $\sin ^{2} \theta$ law obtained with 2 polarizers. The polaroid element $N_{1}$ eliminates the errors due to a contingent polarization of the light source. Monochromatic filters can be added.

We would also mention a very simple device which we have used (Fig. 10.19). It consists of a plastic-material rod (diameter: about $3 \mathrm{~mm}$ ), which conveys the comparison light into the eyepiece. The 
microscope-illuminating source $S$ radiates light in the plastic-material $\operatorname{rod} T$. The light travels in the rod owing to full reflections against its walls. Bending the rod does not alter the phenomena. The com-

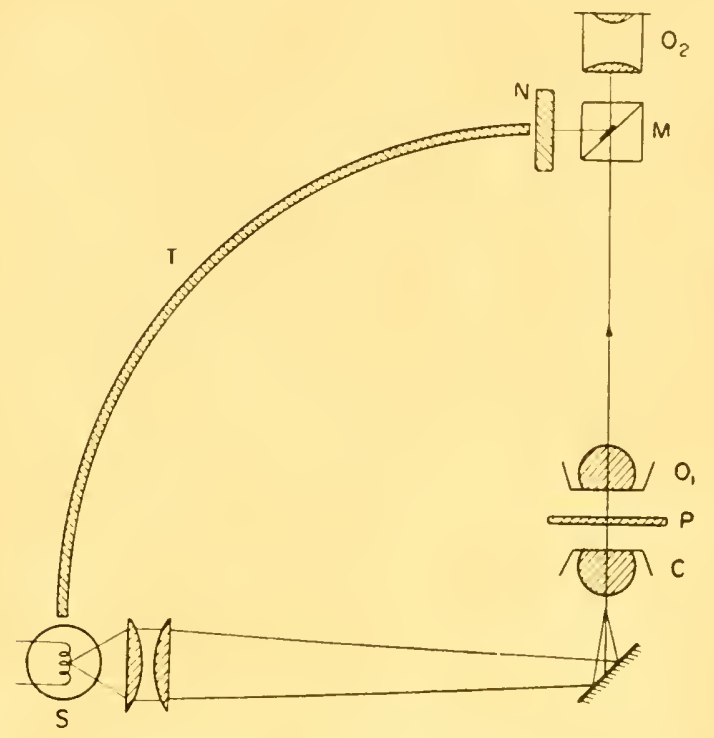

FIG. 10.19. Photometric device using a plastic-material rod (Françon).

parison surface of the cube $M$ is thus readily illuminated. Photometric balance or measurement is made by means of a device, such as neutral wedge or polaroid element. Monochromatic filters can be used.

\section{FLUORESCENCE MICROSPECTROPHOTOMETRY}

Under ultra-violet radiation, some substances radiate visible light (primary fluorescence) whose constitution may provide valuable information regarding the details of the investigated specimen. Other substances treated with fluorescent materials radiate a fluorescence of different spectral constitution (secondary fluorescence) which is well worth investigating.

A monochromatic incident ultra-violet radiation gives rise to fluorescence comprising several types of radiation. The spectial investigation of the fluorescence is made setting the spectrograph next to the microscope in accordance with a layout similar to the one shown in Fig. 10.11. 
According to Stokes's law, the emitted light is of longer wavelength than the exciting light. When excited by the ultra-violet radiation, the fluorescent elements of the specimen emit visible radiation. Hence, all the optical elements beyond the specimen $P$, the microscope and the spectrograph, may be of glass.

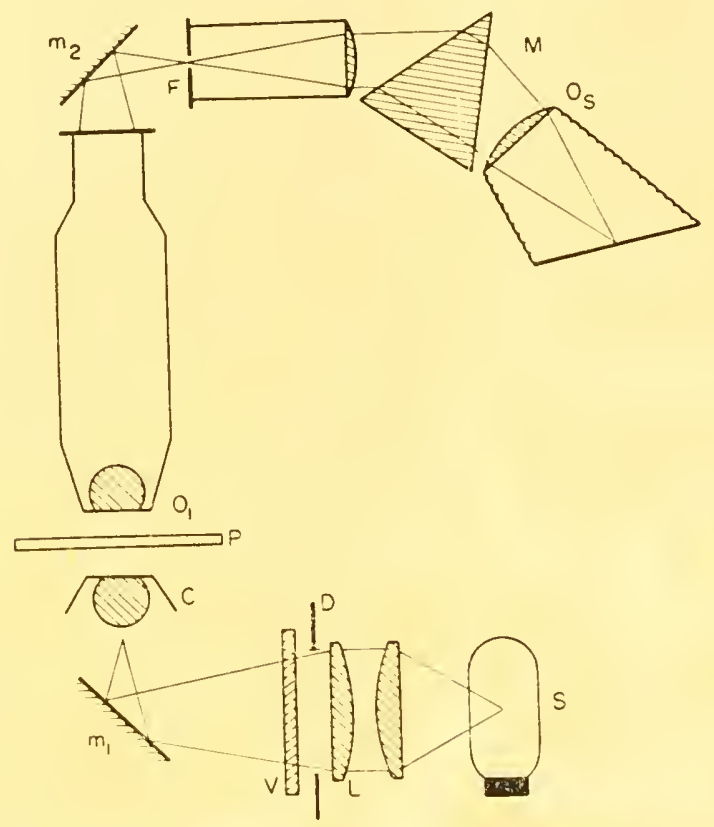

FIG. 10.20. Arrangement for fluorescence microspectrophotometry.

The layout may be the one shown in Fig. 10.20. The source $S$ emits the ultra-violet radiation. By means of the optical system $L, S$ is imaged in the condenser $C$ which is adjusted so as to image the diaphragm $D$ on the specimen (Köhler illumination). The filter $V$. set for instance, close to $D$, occludes the visible light, letting through only the exciting ultra-violet radiation. The optical elements between $S$ and the specimen $P$ should absorb the least possible ultra-violet as, for a given exciting radiation and given observation conditions, the intensity of the fluorescence-emitted light is proportional to the exciting-light intensity. Uviol glass, quartz or reflecting devices are suitable.

As in the layout shown in Fig. 10.11, the specimen $P$ is imaged on the slit $F$ of the spectrograph by the microscope whose objective 
is $O_{1}$. Under the incident ultra-violet radiation, some elements of the objective may become fluorescent. Such fluorescence brings about an undesirable haze which must be occluded by a filter within the objective, which occludes the ultra-violet radiation.

The problem then arising is the following: having obtained the fluorescence spectrum emitted by a detail of the specimen, it is now desired to compare it with that of another detail, either of the selfsame or of another specimen.

The same procedure as used previously achieves this as follows: a portion of the spectrograph slit $F$ is covered by the fluorescent image and the remainder by the image of a comparison source exhibiting a continuous spectrum. A rotatable sector is set on the latter portion which shows, on the photographic plate, a series of continuous spectra correlated to various illuminations whose relative values are thus determined. Exposure time is the same for either the fluorescence spectrum or the comparison spectra. Then, at every wave-length, the curve connecting the measured densities $(d)$ (photographic densities measured on the comparison spectra) to the logarithms of relative

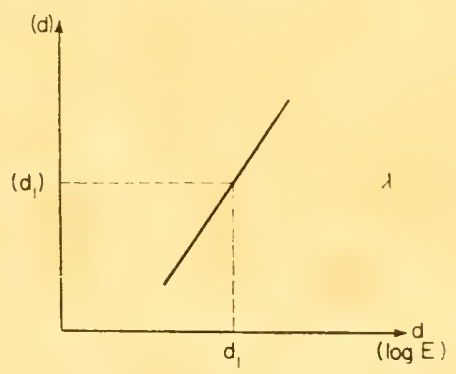

FIG. 10.21. Curve connecting (d) (photographic density) to the absorbance $d$.

illumination can be plotted. Relative-illumination logarithms may be substituted for densities $d$, which may define the illumination of the plate. The higher the density, the greater the illumination received.

Let us now consider a fluorescent detail whose measured density is $\left(d_{1}\right)$. This is the density of the fluorescence spectrum at the wavelength $\lambda$. The curve connecting $(d)$ to $d$ exhibits a density $d_{1}$ (Fig. 10.21). Such density defines the intensity of the fluorescence emitted by the detail at the wave-length $\lambda$. Changing the wave-length enables to plot the curve $d=f(\lambda)$, defining the fluorescence change of the detail versus the wave-length. 
Where it is required to investigate another detail whose fluorescence spectrum is in the same plate, the measured density $\left(d_{2}\right)$ exhibits the characteristic density $d_{2}$ which may be compared direct with $d_{1}$.

When the fluorescence spectrum of a detail is to be drawn from another plate, the comparison spectra are brought about under the same conditions, the same source and the same rotatable sector. Exposure-time is identical for both plates. Then the densities obtained from the latter may be compared.

As typical example of fluorescent microspectrophotometric measurements, Fig. 10.22 shows, according to Sjöstrand, the fluorescence of the sciatic-nerve sheath of a rabbit. The ordinates show the densities $d$

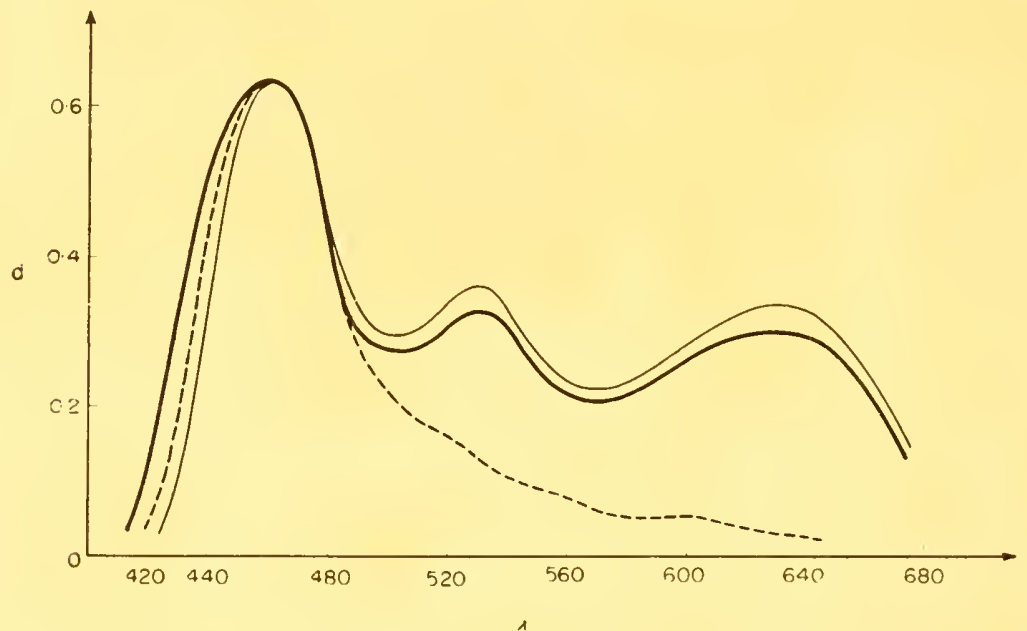

FIG. 10.22. Spectral fluorescence of the sciatic-nerve sheath of a rabbit (after Sjöstrand). - Solution of thiamine, -..-- solution of thiochrome.

just defined. Figure 10.22 shows also the curves obtained with solutions of thiochrome and thiamine, respectively. In this way, Sjöstrand found, in the sciatic-nerve sheath, the emission spectral line at $0.460 \mu$, characteristic of the triochrome. The spectrum also shows lines due to thiamine derivates located in longer wave-length ranges. 


\section{CHAPTER XI \\ Chemical Microscopy}

\section{INTRODUCTION}

GREAT strides have been made in chemical microscopy since the works of L. and A. Köfler. Application of the microscope to thermal analysis provides the means to define and identify an organic compound and, when a mixture is involved, to determine its composition. All phenomena, concomitant with the temperature rise of a substance up to its melting point, such as: sublimation, decomposition, birefringence, polymorphic transformations and so forth, can be studied. This is why the term "fusion methods" is frequently applied to operations taking place during the temperature rise and the cooling of the specimen.

Lastly, microscopic methods require not only very little time but small quantities of substance as well, usually a few milligrammes.

\section{HOT STAGE AND COLD STAGE}

The hot stage is the microscope element devised for application of fusion methods. It is placed on the stage of a standard microscope and designed for heating or cooling the specimen ad libitum.

Figure 11.1 shows the diagrammatic cross-section of the Köfler-type hot stage. The apparatus is of cylindrical shape and has the size of the microscope stage. The object is placed on the object-slide $A$ and encased in a cover-slip. The object-slide is secured by the metal frame $B$ connected to the rod $C C^{\prime}$. The small threaded element $V$ is provided to rotate $C$ about its axis thus shifting $A$ in the direction $C C^{\prime}$. The element $V$ may rotate about a vertical axis so that, when shifting $C$ horizontally, $A$ describes a circular motion centred upon $V$. These two linked motions ensure that the specimen can be brought at any point within the field of view. The upper and lower parts of the heating-chamber are closed by the glass plates $D$ and $G$, respectively. 
The stage is heated by a resistor located at $E$ and $F$. The temperature is measured by a thermometer at $H$, its tip being very close to the specimen. Figure 11.2 shows a hot stage (type Köfler of Reichert), fitted to a microscope. The more widely used types of the stages can be used up to $350^{\circ} \mathrm{C}$. Observation is always carried out at a low
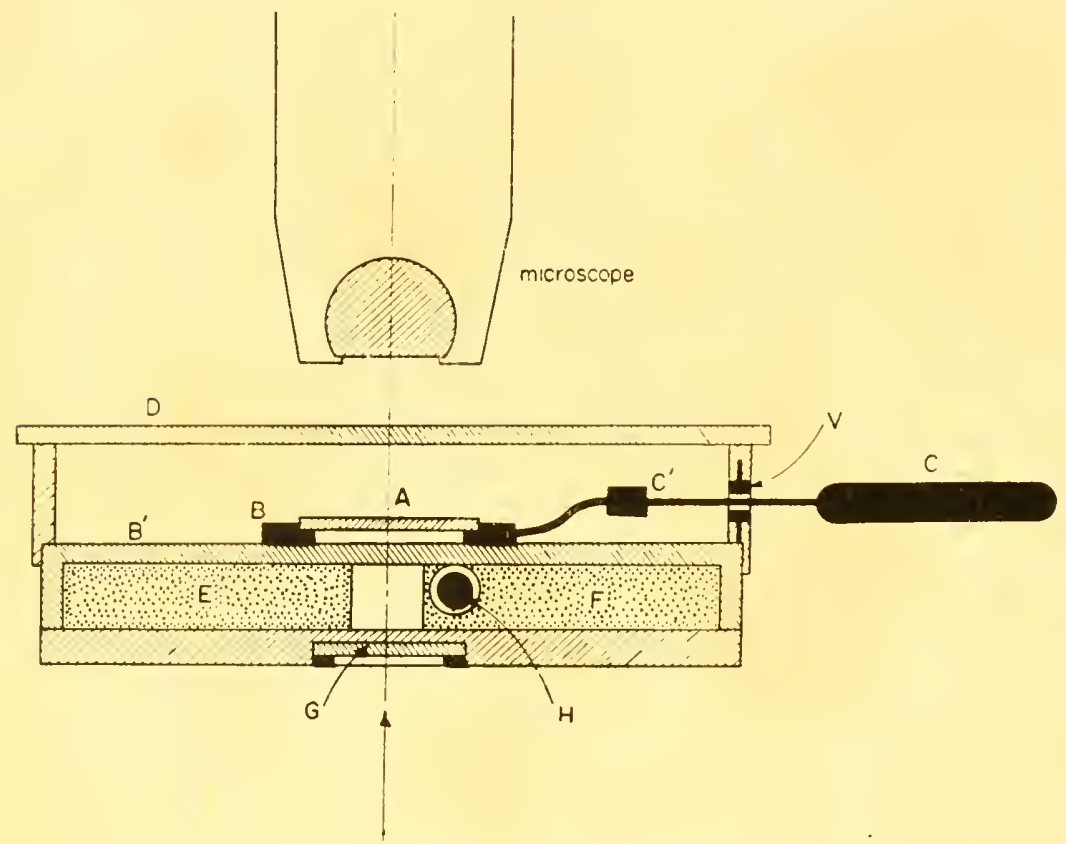

FIG. 11.1. Cross-section through the Köfler $30^{\circ}$ to $350^{\circ}$ hot stage.

magnification and, therefore, objectives with a long working distance can be employed thus preventing the ill effects caused by the temperature rise. The objectives generally employed have a $\times 3, \times 5$ magnification, $\times 10$ at most, associated with eyepieces $\times 10$ or $\times 20$. Therefore, total magnification does not exceed $\times 200$. Except, of course, during observations, the objective is kept away as far as possible from heated specimens in order to prevent any damage. When investigating sublimation, $A$ is substituted for a cap covered with a glass plate which serves as condenser for the substance to be sublimated. Special devices allowing to study low pressure sublimation have been devised.

Cold stages whose minimal temperature is under $0^{\circ} \mathrm{C}$ are valuable for measuring the melting point and studying the polymorphism of organic compounds which are liquid at normal temperatures. 
Low temperatures of $-100,-120^{\circ} \mathrm{C}$ are obtained by circulating in the cold stage a cooled gas such as dry nitrogen, which is cooled in a coil immersed in liquid nitrogen (A. H. Thomas, W. C. McCrone). In most cold stages, carbonic acid snow is used as it readily provides temperatures down to $-60^{\circ} \mathrm{C}$ approx. (Reichert, Leitz).

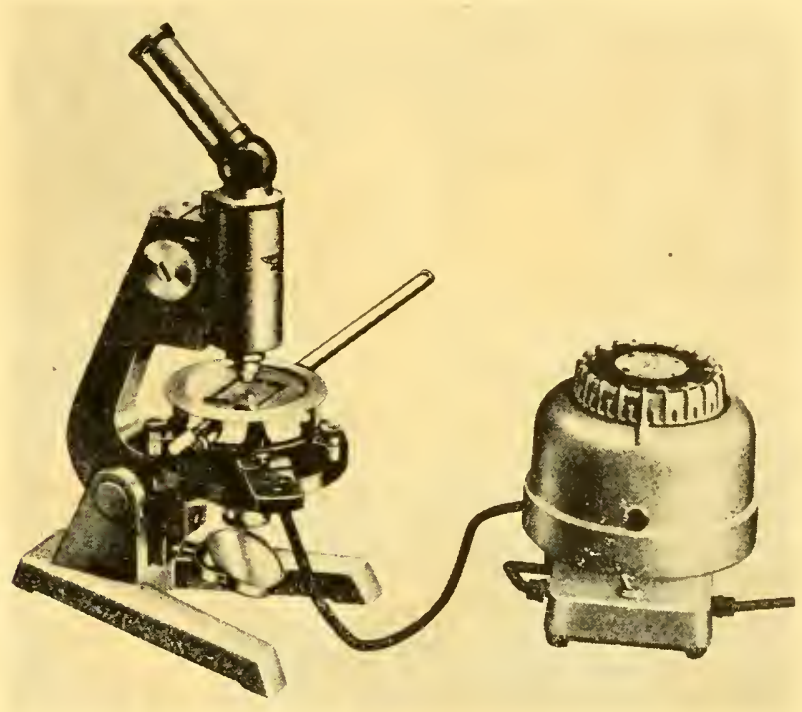

FIG. 11.2. Reichert hot stage microscope.

Inserting a hot stage or a cold stage between the condenser and the microscope objective brings about a gap between these two elements thus frequently precluding the use of a hot or cold stage in conjunction with a phase-contrast or interference microscope. The condenser diaphragm to be imaged on the phase plate in the objective is not always in the focus of the condenser thus preventing coincidence of the diaphragm image with the phase plate. Some hot stage microscopes were designed for phase-contrast and dark-ground applications and also, providing reflected-light observation by means of a vertical illuminator (Reichert). Figure 11.3 shows the O. P. L. microscope, fitted with a hot stage designed for investigating opaque objects.

Peltier-effect applications to hot or cold stages have been made in the U.S.S.R. Let us take two welded metals, $A$ and $B$, and insert these two conductors in series in a circuit through which electric current is flowing. If the current travels in the direction $A B$, either 
heat is developed or heat absorption occurs at the weld (Peltier effect). For instance, if heat is being developed, the heat will be absorbed if the current direction is reversed.

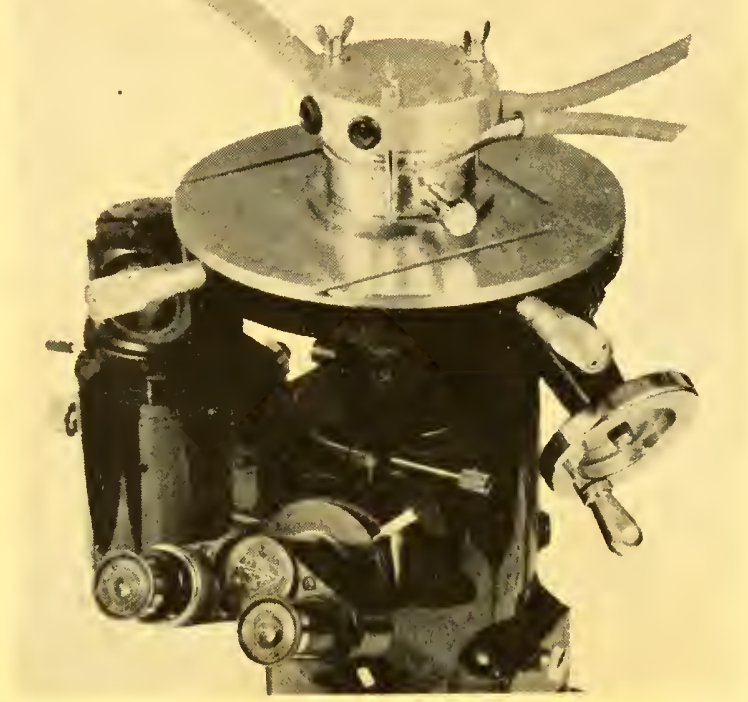

FIG. 11.3. O.P.L. (Optique Precision de Levallois) hot stage microscope designed for investigating opaque objects.

\section{CALIBRATING THE HOT STAGE}

The hot stage is heated by a resistor. The current is rheostatadjusted. Calibration consists in determining, for every rheostat adjustment, the temperature variation versus time.

The procedure is the following: the hot stage (with its ancillaries and a specimen) is set on the microscope, the thermometer being fully inserted into its recess. The rheostat-adjusted potential difference is shown by a voltmeter connected across the hot stage terminals. Then, the rheostat slide is so adjusted that, initially, the potential difference is low, e.g. 30 volts. The temperature is recorded every minute until the temperature rise drops to less than $1{ }^{\circ} \mathrm{C}$ per minute. This shows, therefore, in relation to the potential difference considered, temperatures for which the rise is $1^{\circ}, 2^{\circ}, 3^{\circ}, \ldots$ per minute. The foregoing measurements are resumed for several potential differences, and curves, 
similar to those in Fig. 11.4, are derived. In this graph, the temperatures read off the thermometer are shown as ordinates and the potential differences as abscissae. Two curves are plotted: the first corresponds to a temperature rise of $2^{\circ} \mathrm{C}$ per minute, the other $4^{\circ} \mathrm{C}$

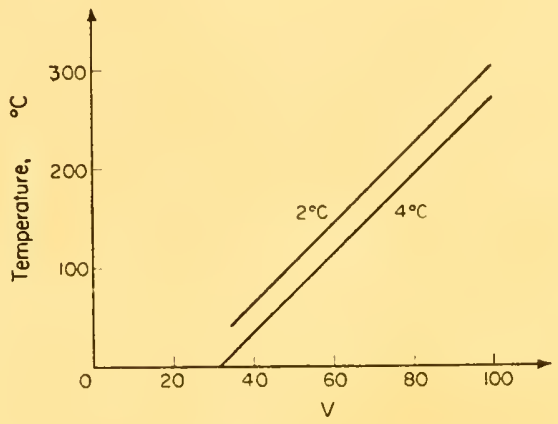

FIG. 11.4. Calibrating the hot stage.

per minute. If a temperature rise of $2^{\circ} \mathrm{C}$ per minute is required at $200^{\circ} \mathrm{C}$ approx., the graph shows that the rheostat adjustment is to provide a 73-volt potential difference.

\section{DETERMINING THE MELTING POINT}

Before attempting measurements of undetermined substances, it is indispensable to find the melting points of several compounds the melting point of which is known. After putting several crystals of the substance on the object-slide, and covering the latter with the coverslip, the cover-slip is rotated by means of, for example, an india-rubber eraser, thus spreading out the sample since the crystals adhering to the cover-slip underface melt at approximately $1^{\circ}$ above the melting point of the crystals on the object-slide, the best procedure is to remove the latter, clean it and re-set it on the specimen. In this way, the crystals are on the object-slide and not on the cover-slip under-face.

All the accessories being in order, focusing is made, selecting an area of the specimen where discrete crystals are discernible. The rheostat is adjusted so that temperature rises promptly to about ten degrees below the melting point. Heating is stopped until temperature begins to drop. Using calibration curves similar to those shown in Fig. 11.4 , heating is resumed so that temperature rises about $2^{\circ}$ per minute. When $2^{\circ}$ below the melting point, the rate of temperature 
rise is slightly decreased. Shortly before melting the crystals lose their shape, their outlines rounding off. The temperature at which the last crystal melts is considered as the melting point.

When dealing with an unknown substance, the melting point is determined approximately in a preliminary test, followed by the foregoing procedure. In most organic crystals, transition from the solid

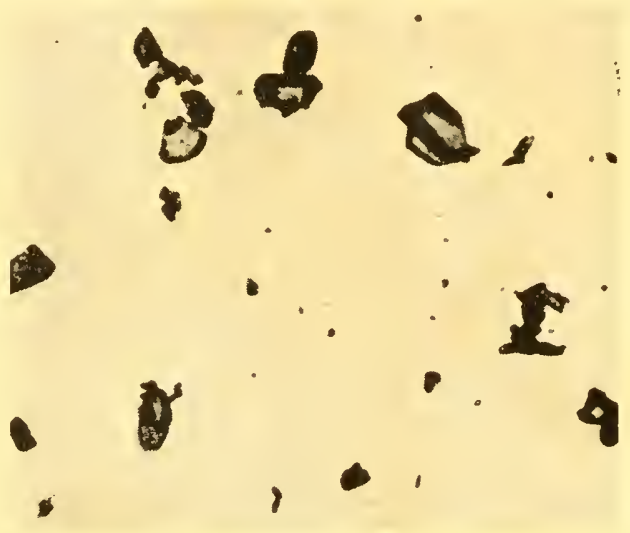

FIG. 11.5. Melting of azobenzene crystals.

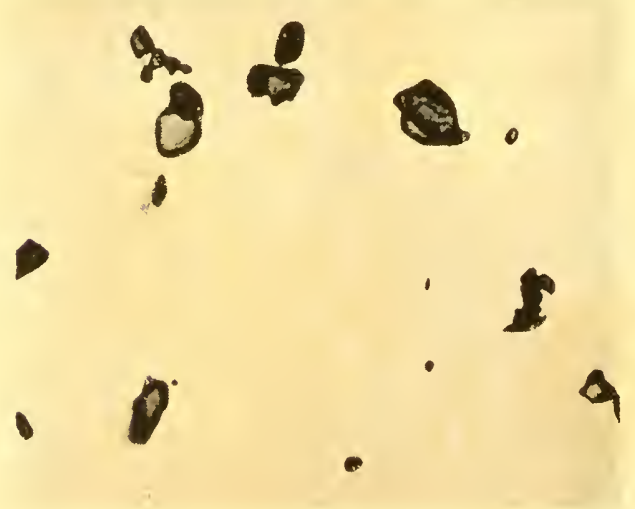

FIG. 11.6. Melling of azobenzene crystals.

to the liquid state is well defined and leaves no room for uncertainty. Melting is to occur at a definite point. If melting extends over several degrees, this means that the substance is not pure. The photographs $11.5,11.6$ and 11.7 show the melting of azobenzene crystals. 
As most crystals are anisotropic, the melting point can be determined by means of polarized light. To carry this out, a polarizer is set under the hot stage and an analyser on the eyepiece, the work taking place between polarizer and crossed analysers. The birefringent crystals show up bright against a dark ground. When melted, they vanish and the whole field becomes dark. Vanishing of birefringence

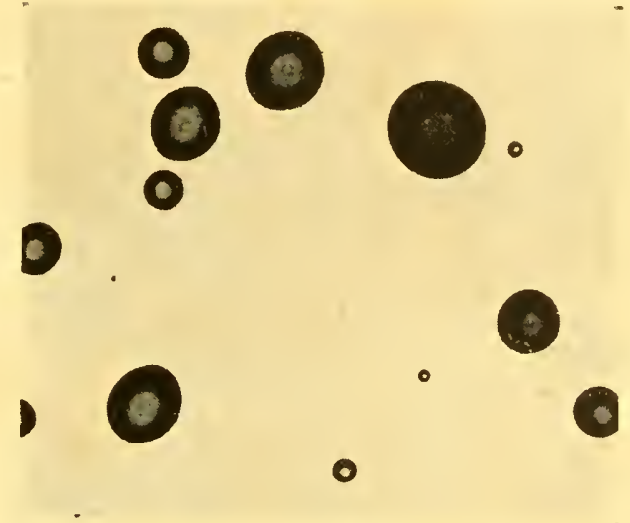

FIG. 11.7. Melting of azobenzene crystals.

determines the melting point. However, it may occur that a crystal is no longer birefringent at a temperature lower than its melting point. It may yield an isotropic polymorph (carbon tetrabromide becomes isotropic at $46.9^{\circ}$ and melts at $92.5^{\circ}$ ), or become an opaque mass of disorientated crystals of a polymorph or, yet, lose the crystallization solvent and become opaque.

\section{MEASURING THE REFRACTION INDEX OF A MELTED SUBSTANCE}

The refraction index is a useful datum for defining pure compounds or analysing chemical binary systems.

Köfler showed a method, based on glass powders, whose indices are known, for measuring the refraction index of a melted substance.

A specific powder is mixed with the substance in the solid state. A few pellets of the mixture are then put on the hot stage and observed through the microscope. As temperature rises, the crystals melt and the glass particles are visible, immersed in the liquid. Köfler makes use of Becke's line (see Chapter VII, $\S 4$ ) to determine the phase showing the higher-index. The work is effected in coherent light by reducing 
the condenser aperture and Becke's line is observed by shifting the microscope setting. This line shifts to the higher-index phase when the microscope is moved away from the specimen. In order to eliminate dispersion effects, a monochromatic filter is used. Measuring the index of the melted substance consists in causing the glass particles to vanish by suitable temperature adjustment. At the temperature considered and the wave-length used, the melted substance and the glass particles have the same refraction index. In most types of glass. the index increases by one unit of six places of decimals per degree centigrade $\left(10^{-6}\right)$. It may therefore be considered that the glassparticle index remains constant from 50 to $300^{\circ} \mathrm{C}$. Conversely, the index of melted organic substances varies much more. Such variations may attain and even exceed $10^{-3}$ per degree. Rising temperature lowers the index. Indices are balanced by increasing the temperature if the melted substance has a higher index than that of the glass particles. If the index of the melted substance is lower, the powder is to be changed.

As temperature rises, some compounds may be sublimated or decomposed, this making it difficult to determine the refraction index. Measurements are yet feasible if the compound can be supercooled. Once the substance has melted, the hot stage is cooled in order to achieve supercooling. The resulting liquid has a lower temperature than the melting point and its index is therefore higher than that of the non-supercooled liquid. Then, equalization can be made with high-index glass particles.

The foregoing procedure requires temperature variations substantially above the melting point. In some cases, such variations make the method inapplicable.

The refraction index can be measured, using interference methods without calling for temperature adjustments. First, the index for the temperature of the specimen may be determined. If the temperature varies, the corresponding variations of the refraction index can be followed up. Polarizing interference methods, in conjunction with the Köfler process, are particularly simple to apply (see Chapter VII. $\$ 8$ ).

\section{MIXED FUSION - KÖFLER'S CONTACT METHOD}

In the contact method, devised by Köfler, the mixing area of two substances in contact between the slide-object and the slip-cover is observed. This method is very suitable for studying the purity of 
a crystallized organic compound and determining whether two compounds are identical.

The process is the following (Fig. 11.8(a), (b), (c)). One to two milligrammes of the least fusible substance $B$ are melted first. The substance is set against the slip-cover, is flame-melted and spreads under it. Good care is taken that the melted substance does not

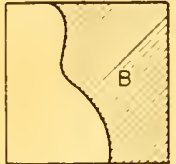

(a)

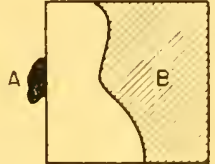

(b)

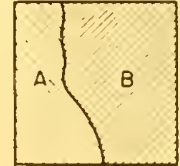

(c)

FIG. 11.8. Mixed fusion - Köfler's contact method.

extend over more than one half of the surface of the specimen (Fig. 11.8(a)). When $B$ has cooled off and solidified, the second substance $A$ (Fig. 11.8(b)) is heated until it melts, spreads under the slipcover and comes in contact with $B$ (Fig. 11.8(c)). If, during this process, a portion of $B$ were cooled, the whole is allowed to crystallize again.

\section{(a) The substances $A$ and $B$ are identical}

In this case, there is no mixing zone. Crystals grow throughout the specimen until the whole is solidified. The mixing zone exhibits no discontinuity either in the growth or shape of the crystals.

(b) The substances $A$ and $B$ are not identical

If the substances $A$ and $B$ are not identical, a break comes out in the mixing area.

(i) The two substancès do not react and are miscible in any proportion. If the substances $A$ and $B$ are not identical a break appears in the mixture area. In this case, they may furnish a continuous series of mixed crystals whose properties change continuously within the range of limit values corresponding to the forms of $A$ and $B$. Plotting the temperature as ordinates, and the mixture composition as abscissae, gives the curve shown in Fig. 11.9. The dotted-line curve shows the initial melting temperature and the full-line curve the temperature when the melting process is over. During the cooling process only one kind of mixed crystals is deposited. Under the graph is shown a diagrammatic aspect of the specimen when heated at a temperature 
slightly above the melting point of the more fusible substance $A$. In the case shown in Fig. 11.9 melting begins at the left and gradually extends to the right.
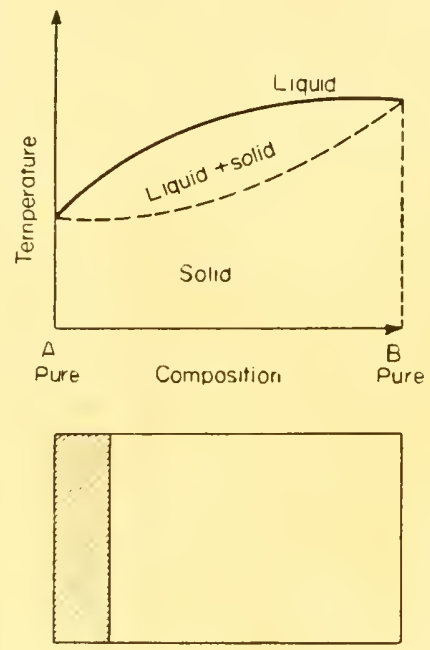

Preparation

Fig. 11.9. Temperature-composition diagram. The substances $A$ and $B$ are miscible in any proportions (type I).

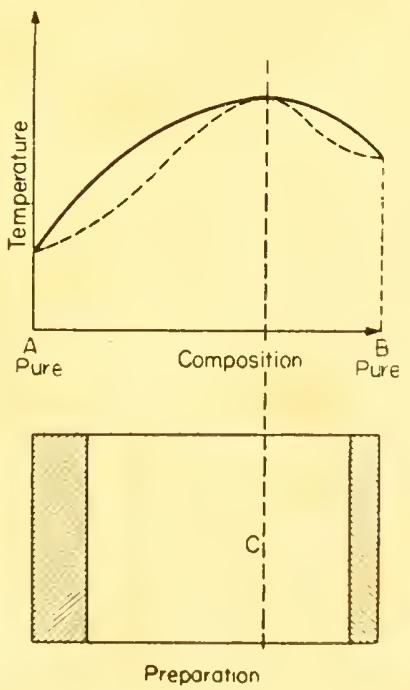

FIG. 11.10. Temperature-composition diagram. The substances $A$ and $B$ are niscible in any proportions (type II). 
Experience has revealed that there may be two other types o1 diagrams: they are shown in Figs. 11.10 and 11.11. As temperature of the specimen rises (as shown in Fig. 11.10), melting extends first

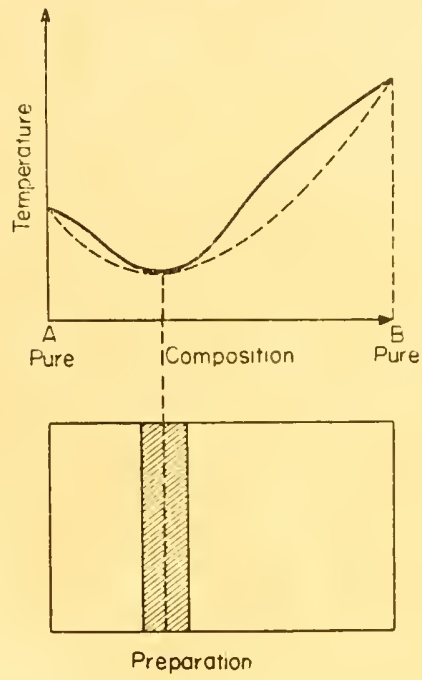

FIG. 11.11. Temperature-composition diagram - The substances $A$ and $B$ are miscible in any proportions (type III).

from the left, then from the right to reach the $C$ area. When the curves evince a minimum (Fig. 11.11), melting is initiated in the area corresponding to that minimum and gradually extends on either side.

(ii) The two substances do not react and formation of a eutectic mixture occurs. In this case, the crystals of the pure substances $A$ and $B$ grow on either side up to the contact zone where the eutectic is developed. Figure 11.12 shows the composition-temperature diagram and the aspect of the specimen when the temperature slightly exceeds the eutectic point. As the diagram shows, the eutectic that melts first is in the area $E$. The eutectic, between crossed polarizers, looks like a dark strip. The melting point of the eutectic was used by the Köflers as an additional datum enabling them to characterize organic compounds.

(iii) Both substances react. The definite compound $M$ is formed in the contact area. Figure 11.13 is derived by placing side-by-side the diagrams related to the systems $A M$ and $M B$, diagrams which are the same as those in Fig. 11.12 but the point $M$ corresponding to the definite compound, is shown by a rounded maximum. 
As the solidified specimen is heated, the two eutectics, $E_{1}$ and $E_{2}$, melt. The eutectic $E_{1}$ is between the substance $A$ and the compound $M$, the eutectic $E_{2}$ being between the compound $M$ and the substance $B$. If the temperature is increased, the compound $M$ melts and

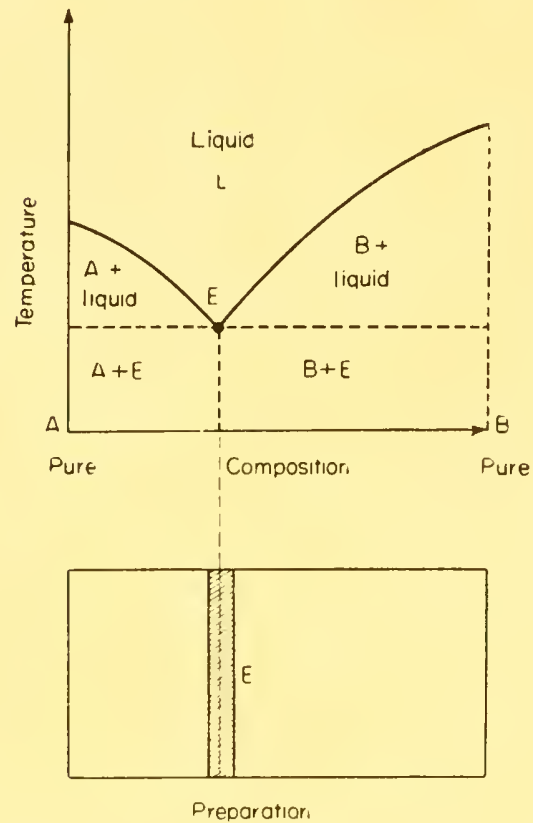

FIG. 11.12. Binary temperature-composition diagram in a system showing simple eutectic formation.
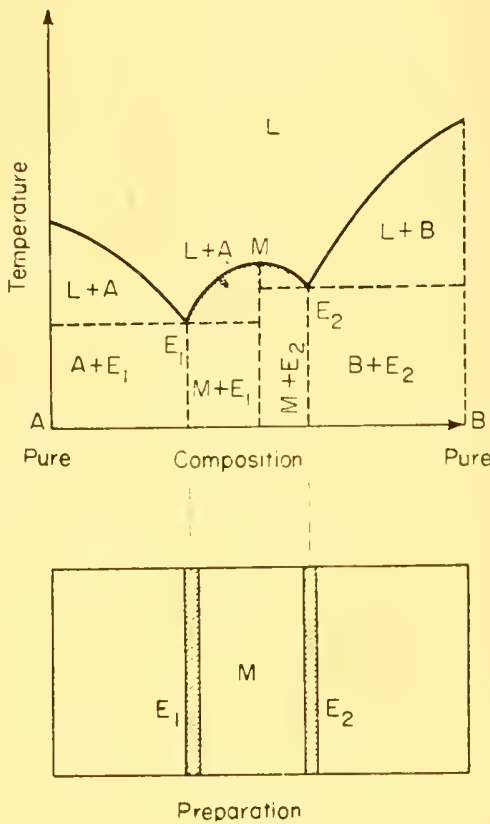

Fig. 11.13. Temperature-composition diagram for an addition compound system (congruently melting addition compound).

becomes a homogeneous liquid: fusion is congruent. In incongruent fusion, the compound $M$ cannot melt without being decomposed.

(iv) The substances $A$ and $B$ do not react and are not miscible in any proportion in the solid state. When the substances $A$ and $B$ are not miscible in any proportion in the solid state, they yield two series of mixed crystals, some are richer in $A$, some are richer in $B$ as shown in Fig. 11.14 and 11.15, respectively. In the first instance (Fig. 11.14), the series of mixed crystals richer in $A$ have a $B$ content ranging from zero to the value shown by the abscissa of the point $C_{1}$. The crystals richer in $B$ evince their saturation limit in $A$ (abscissa of the point $C_{2}$ ). The inter-space $C_{1} C_{2}$ is a lack of miscibility, in the solid state, of the two substances $A$ and $B$ (lack of syncrystallization). The saturated 
crystals $C_{1}$ and $C_{2}$ are called limit mixed crystals. In Fig. 11.14, $(A)$ and $(B)$ denote the isomorphic crystals of the constituent $A$ and $B$, $\left(C_{1}\right)$ and $\left(C_{2}\right)$ the limit mixed crystals. Figure 11.15 does not differ much from Fig. 11.12. The mixtures on either side of the point $K$
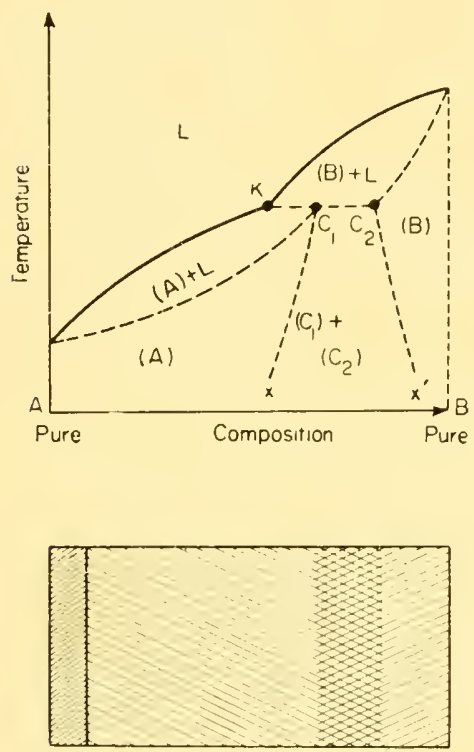

Preparation

FIG. 11.14. Temperature-composition diagram when substances $A$ and $B$ are not miscible in any proportion in the solid state (peritectic point $K$ ).
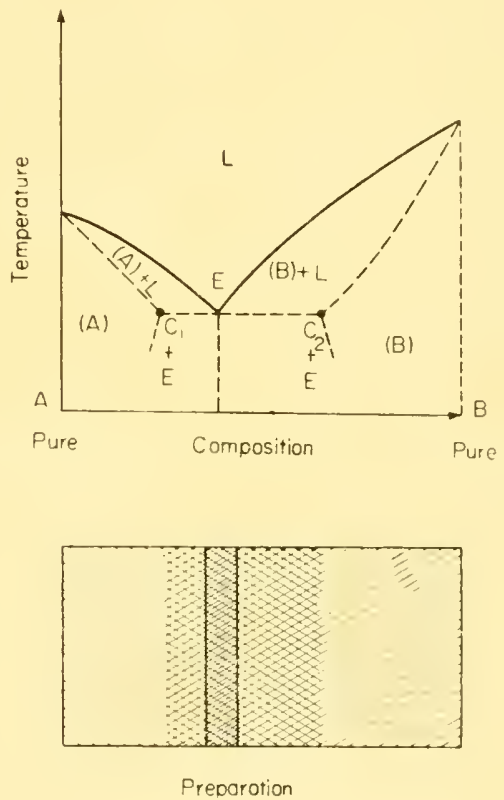

FIG. 11.15. Temperature-composition diagram when substances $A$ and $B$ are not miscible in any proportion in the solid state (eutectic point $E$ and mixed crystals).

(peritectic point) are similar to those shown in Fig. 11.12. The area to the left is the first one to melt as temperature rises. A simple eutectic is shown in Fig. 11.15, the $A$ crystals having a low $B$ content and conversely. The points $C_{1}$ and $C_{2}$ correspond to the limit mixed crystals which are isomorphic to $A$ and $B$, respectively.

\section{TEMPERATURE COMPOSITION DIAGRAM OF BINARY MIXTURES}

In general, plotting of the temperature composition diagram merely consists in determining the melting points $M$ and $N$ of the two pure substances $A$ and $B$ (Fig. 11.16) and both the melting point and com- 
position of the eutectic mixture $E$. The fusion curves $M E$ and $N E$ are plotted arbitrarily. If required, the melting point and composition of an addition compound, the maximum or minimum of the curves of a solid solution may also be determined.

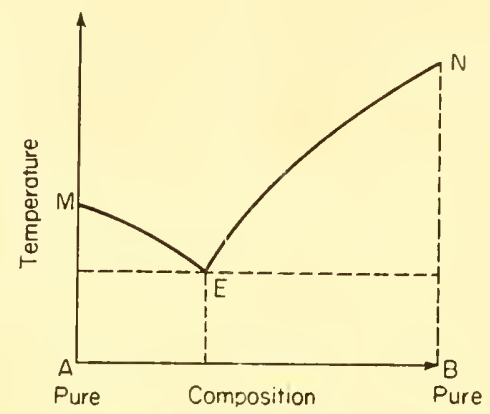

FIG. 11.16. Köfler method for determination of eutectic composition.

Köfler devised a striking method whereby the temperature composition of a binary mixture can be promptly determined without any need for weighing. Let us assume that the index of pure $A$ be 1.350 at $t_{1}^{0}$ and the index of pure $B$ be identical at $t_{2}^{0}$ (Fig. 11.17). In most melted mixtures and liquid bodies at normal temperature,

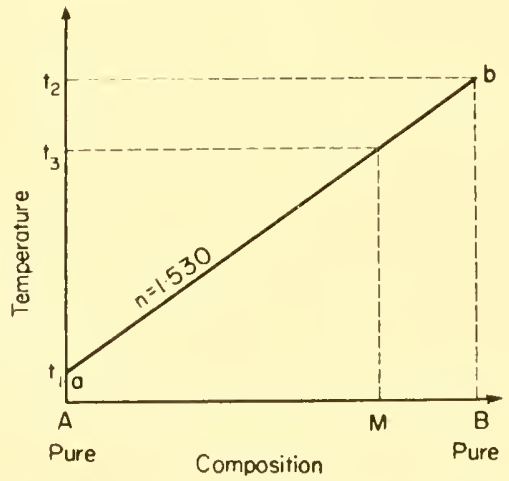

FIG. 11.17. Temperature variation versus the mixture composition kecping the index constant.

the temperature variation versus the mixture composition keeping the index constant (1.530 in the case depicted in Fig. 11.17), is shown by a straight line, such as $a b$. As an instance, for a mixture of composition $M$, the mixture index is 1.530 at the temperature $t_{3}^{0}$. This 
result was employed by the Köflers to restrict determination of the temperature-composition diagram to index measurements using the glass-powder method. First, the glass powder is inserted between the object-slide and the cover-slip, the contact method being prepared

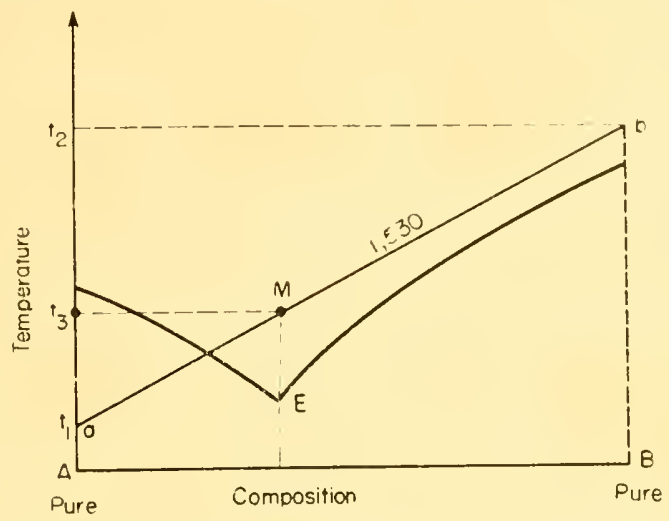

FIG. 11.18. Determination of eutectic point composition.

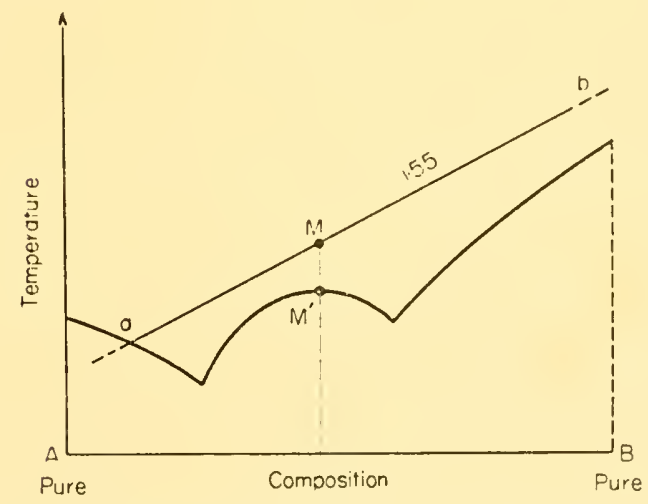

FIG. 11.19. Determination of addition compound composition (M).

as usual. Let us now determine the eutectic point. Next, the specimen is heated on the hot stage and, as the glass particles are scattered throughout the specimen, some of them are always present in the eutectic area. One such particle is observed as the eutectic is melting. If the glass-particles index is adequate, a time comes, as temperature rises, when the particle is no longer visible, i.e. its index is the same as the liquid's, this corresponding to point $M$ in Fig. 11.18. At the temperature $t_{3}^{\prime \prime}$, the melted eutectic mixture has an index of 1.530 . 
Balancing the indices thus allows one to determine the point $M$ along the straight line $a b$. The abscissa of $M$ shows the composition of the eutectic mixture. Adding to this measurement that of the eutectic's melting point, the position of the point $E$ on the diagram is determined. Procedure is the same with an addition compound (Fig. 11.19). Having

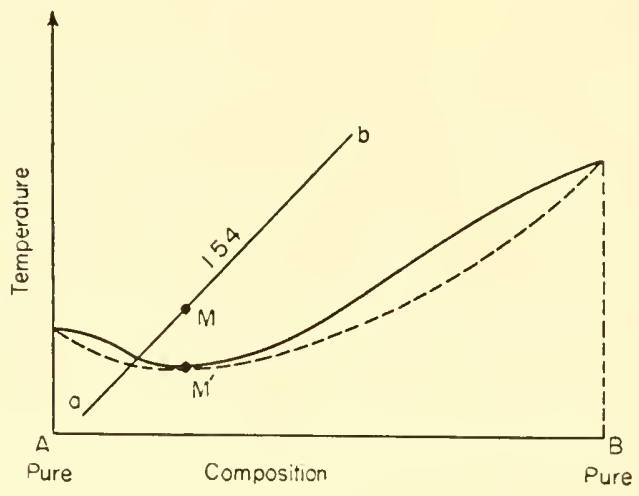

FIG. 11.20. Determination of minimum melting composition $\left(\boldsymbol{M}^{\prime}\right)$.

previously determined the straight line $a b$ with $A$ and $B$ being pure. the point $M$ is sought by balancing the particles index with the melted addition compound. Composition of a maximum and a minimum of the fusion curve may be determined in this way (Fig. 11.20).

\section{APPLICATION OF FUSION METHODS}

Observations are made during the temperature rise and cooling of the specimen and, next, after crystallization.

Experiments are complemented by application of the Köfler contact method. We will now set forth, in accordance with the foregoing, the more important properties that may be investigated together with the feasible measurements when applying fusion methods.

(a) Observations made as the specimen temperature rises

(i) The compound is being sublimated. Observation of the characteristics of the crystals formed.

(ii) A break in certain physical properties is evinced: a polymorphic transformation is taking place. For instance, carbon tetrabromide changes over from the monoclinic form to the cubic form at $46 \cdot 9^{\circ} \mathrm{C}$. 
(iii) The substance decomposes.

(iv) The substance melts. Determination of its melting point.

(v) Measurement of the refraction index of the melted substance.

(b) Observations made as the specimen cools off

(i) Supercooling is taking place: the compound remains liquid below its melting point.

(ii) Observation of the rate of crystallization.

(iii) Observation of the crystallization front. As crystallization extends into the specimen, the boundary between the solid and liquid areas may exhibit three aspects:

$(\alpha)$ The front is smooth and uniform-orientated areas are forming (naphthalene);

( $\beta$ ) Formation of discrete and variously shaped crystals (picric acid);

$(\gamma)$ The crystallization front consists of small spherules which are developing from discrete nuclei (cholesteryl acetate). The shape of the crystallization front depends on substance purity and temperature. If the substance exhibits a polymorph, the latter does not exhibit the same crystallization front.

(iv) A second crystallization takes place after normal crystallization of the melted substance: this is a polymorphic transformation (carbon tetrabromide).

(c) Observations made after the specimen is crystallized

(i) Nature of the crystals formed.

(ii) Birefringence - Observations in both parallel and converging light.

(d) Observations made applying the contact method using a reference substance

(i) Determining whether the investigated substance $A$ is identical with the reference substance $B$, thus making $A$ identifiable. When $A$ and $B$ are identical, crystals develop throughout the specimen, without giving rise to a mixed area.

(ii) Plotting the composition-temperature diagram. Solid solution. Mixture with formation of a eutectic. Melting point and measurement of the refraction index of the melted eutectic. Formation of an addition compound. 


\section{CHARACTERIZING AND IDENTIFYING FUSIBLE COMPOUNDS}

The Köflers have published tables showing the data of 1200 organic compounds including melting point, temperature of eutectic point with two reference substances and refractive index of the melted substances. These numerical data are complemented with the description of many properties recorded during the survey of the compounds. These tables are used to identify the fusible compounds by comparing the observations and measurements derived from fusion methods with the data shown in the tables. The Köfler tables were gone over, in a very convenient form, by W. C. McCrone, in his work: "Fusion methods in chemical microscopy". 


\section{SHORT BIBLIOGRAPHY}

Alpern, B. and Nomarski, G. (1954) Contribution à l'amélioration des méthodes d'examen microscopique des charbons. Bull. Soc. Min. Crist. 77, 905.

BARER, R. (1952) Combined phase-contrast and interference-contrast microscopy. Nature, Lond. 169, 108.

BARER, R. (1955) A vector theory of phase-contrast and interference-contrast. Micr. Soc. $75,23$.

BARER, R. (1955) Les applications de la microscopie et de la microspectroscopie à la chimie biologique. Chim. Anal. 37, No. 10, 327-337.

BARER, R. (1955) Phase-contrast, interference-contrast and polarizing microscopy. Analytical Cytology. McGraw-Hill, N. Y.

BARER, R. (1956) Phase-contrast Microscopy. Modern Methods of Microscopy. Butterworths, p. 66.

BARER, R. and Dick, D. A. T. (1957) Interferometry and refractrometry of cells in time tissue culture. Cytochenical Methods with Quantitative Ains. Academic Press. N. Y., p. 103.

BARER, R., Holiday, E. R. and Jope, E. M. The technique of Ultraviolet Absorption Spectroscopy with the Burch Reflecting Microscope. Biochim. biophys Acta 1, 123-134.

BENDFORD, J. R. and SEIDENBERG, R. L. (1S5) Phase-contrast microscopy for opaque specimens. J. Opt. Soc. Amer. 40, No. 5, 314.

Bennett, A. H., Jupnik, H., Osterberg, H. and Richards, O. W. (1951) Phase Microscopy, Principles and Applications. Wiley, N. Y.; Chapman and Hall.

Bennett, A. H., Woernley, D. L. and Kavanagh, A. J. (1948) Ultraviolet phase microscopy. J. Opt. Soc. Amer. 38, 739.

Blout, E. R. (1949) Microspectroscopy. Physical Methods of Organic chemistry. Interscience N. Y., p. 2179.

Blout, E. R., Bird, G. R. and Grey, D. S. (1950) Infrared microscopy. J. Opt. Soc. Amer. 40, No. 5, 304-313.

Bouwers, A. (1946) Achievements in Optics. Elsevier, Amsterdam.

BRUMBERG, E. M. (1943) Colour microscopy in ultraviolet rays. Nature, Lond. 152, 357.

Burch, C. R. (1947) Reflecting microscopes. Proc. Phys. Soc. Lond. 59, 41.

Burch, C. R. (1947) Semi-aplanat reflecting microscopes. Proc: Phys. Soc. Lond. 59, 47.

CASPERsson, T. (1955) Quantitative Cytochemical Methods for the Study of Cell Metabolism. Experientia 11, No. 2, 45-60.

Chamot, E. M. and MaSOn, C. W. (1958) Handbook of Chemical Microscopy. Wiley, N. Y. MCCrone, WAlter C. (1957) Fusion Methods in Chemical Microscopy. Interscience, N. Y. Cuckow, F. W. (1947) Phase-contrast in the photography of metals. Nature, Lond. 159, 639.

DAvies, H. G. (1958) The determination of mass and concentration by microscope interferometry. General Cytochemical Methods. Academic Press. N. Y. p. 55. 
DYSON, J. (1949) A unit-magnification optical system for the attainment of long working distances in microscopy. Proc. Phys. Soc. Lond. 62, 565.

Dyson, J. (1950) An interference microscope. Proc. Roy. Soc. A204, 170.

Dyson, J. (1952) An interferometer for opaque objects. Proc. Roy. Soc. A216, 493. ELIAS, C. (1959) Interferencia por polarizaçao aplicada à medidas dos indices de refraçao em microscopia. Instituto de Biofisica, Rio de Janeiro.

FAUST, R. C. (1952) Multiple beam interference microscopy. Proc. Roy. Soc. 11,2 240.

Foster, L. V. (1938) Polarizing vertical illuminator. J. Opt. Soc. Aner. 28, 124.

FRANÇON, M. (1952) Contraste de phase et contraste par interférences. Rev. Opt. (théor. instrum.).

FRANÇON, M. (1954) Le microscope à contraste de phase et le microscope interférentiel. Editions du Centre National de la Recherche Scientifique, Paris.

FRAnÇON, M. (1957) Polarization apparatus for interference microscopy and macroscopy of isotropic transparent objects. J. Opt. Soc. Anet. 47, 528.

Frederikse, A. M. (1933) Mikroskopische Beobachtung lebender Zellen. Acta. Brev. Neerl. Physiol. 3, 121.

Gabler, F. (1952) Das Phasenkontrastverfahren und seine Anwendung in der Anflichtmikroskopie. Mikroskopie, 7, 43.

GABLER, F. (1953) Moderne Methoden der Mikroskopischen Untersuchung von Metalloberflächen. Metall 7, 401.

Goodbody, A. M. (1957) The influence of condenser aperture on the resolution of a pair of parallel lines. Proc. Phys. Soc. Lond. 70, 361.

GrehN, J. (1959) Das Durchlicht-Interferenz-Mikroskop als ein Instrument des Biologen. Leitz-Mitteilungen für Wissenschaft und Technik. Band I, Heft 2, page 35.

GrEY, D. S. and LEE, P. H. (1949) New series of microscope objectives. J. Opt. Soc. Amer. 39, pp 719, 723.

Grey, D. S. (1950) New series of microscopes objectives. J. Opt. Soc. Amer. 40, 283. Hartley, W. G. (1947) A variable phase-contrast system for microscopy. Nature, Lond. 159, 880 .

Hopkins, H. H. (1953) A note on the theory of phase-contrast images. Proc. Phys. Soc. Lond. 66, 331.

INGElSTAM, E. and Johansson, L. P. (1958) Correction due to aperture in transmission interference microscopes. J. Sci. Instrmm. 35, 15.

JElley, E. E. (1949) Microscopy. Physical methods of Organic Chemistry. Interscience, N. Y., p. 847.

JOHANSSON, L. P. (1957) An interference microscope for rapid measurements of biological objects. Cytochemical methods with quantitative aims. Academic Press, N. Y., p. 158. JoHnson, B. K. (1949) A compound reflecting microscope of high aperture for use in ultraviolet light. J. Sci. Instrum. 26, 148.

JoHnson, B. K. (1949) An achromatic microscope objective for use with ultraviolet light. J. Sci. Instrmut. 26, 193.

JupNik, H., Osterizerg, H. and Pride, G. E. (1946) Phase microscopy with vertical illumination. J. Opt. Amer. Soc. 36, 710.

KASTIER, A. and MONTARNAL, R. (1948) Phase-contrast in polarized light. Narure, Lond. 161, 357.

KING, R. J. and ROE, E. M. F. (1954) Photomicrography with the Burch reflecting microscope. J. R. Micr. Soc. 74, 64. 
KING, J. (1956) Fluorescence microscopy. Modern methods of nicroscopy. Butterworths, p. 43.

Kofler, L. and Kofler, A. (1954) Mikroskopische Methoden. Handbuch der Mikrochemischen Methoden. Springer Verlag, Wien.

KRUG, W. and LAU, E. (1951) An interference microscope for observations in transmitted and vertical illumination. Ann. Phys. Lpz. 8, 329.

Land, E. H., Blout, E. R., Grey, D. S., Flower, M. S., Husek, H., Jones, R. C., MAtZ, C. H. and MerRill, D. P. (1949) Color translating ultraviolet microscope. Science 109, 371.

LEBEDEFF, A. A. (1930) Interferometre à polarisation et ses applications. Rev. Opt. (théor. instrum.) 9, 385.

Lison, L. (1953) Histochimie et Cytochimie Animales, Principes et Méthodes. GauthierVillars, Paris.

LoCQuin, M. (1951) Deux dispositifs à contraste de phase variables et colorés. J. Phys. Radium 12 (1), 99.

Lohmann, A. (1952) Eine neue Anordnung für variablen Phasenkontrast. Phys. Verh, Mosbach, 3, 200.

Maksutov, D. D. (1932) U.S.S.R. Patent No. 40, 859.

MARTin, L. C. (1951) The elementary theory of phase-contrast. J. Quekett Micr. Cl. 3, 237.

MENZEL, E. (1951) Mehrfacher mikroskopischer Lichtschnitt. Naturwissenschaften (38) heft 14, page 332 .

MENZEL, E. (1957) Dickenmessung mikroskopischer Objekte mit dreispaltund ZweispaltInterferenzen. Optik, p. 151.

Merton, T. (1950) On interference microscopy. Proc. Roy. Soc. 191, 1.

Moтt, B. W. (1956) Metallurgical aspects of microscopy. Modern Methods of Microscopy. Butterworths, p. 59.

Nomarski, G. (1955) Microscopie dans l'infrarouge. Rev. Opt. (théor. instrum.) 34, No. 1, 29.

Nomarski, G. (1955) Microinterférométrie différentielle à ondes polarisées. J. Phys. Radium 16, 9 (S).

NorRIS, K. P. (1956) Development of reflecting microscopes. Modern Methods of Microscopy. Butterworths, p. 15.

Oettlé, A. G. (1950) Experiments with a variable amplitude and phase microscopy. J. R. Micr. Soc. 70, 232.

Osterberg, H. (1947) The polanret microscope. J. Opt. Soc. Amer. 37, 726.

PAYNE, B. O. (1950) A phase-contrast microscope with variable amplitude and phase. J. R. Micr. Soc. 70, 225.

PERRY, J. W. (1950) Surface micro-interferometry. Modern Methods of Microscopy. Butterworths, p. 52.

RAMSAY, J. V. (1951) A phase contrast method for the study of the state of polish of single glass surfaces. J. Sci. Instrum. 28, 24.

Richards, O. W. (1950) Microscopy, fluorescence. Medical Physics, vol. 1I. Year Book Publishers, Chicago, p. 530.

Rienitz, J. (1952) Durchlicht-Interferenzmikroskopie im weissen Licht. Optik 9, 436. RiEnitz, J. (1957) Ein Verfahren zur Verdoppelung der Messgenaingkeit Ermittlung des "stoffeigenen Phasensprungs" und Prüfung der Aldrucktrene in der Anflichtinterferenzmikroskopie. Internationalen Kolloquiums der Hochschule für Elektrotechnik ilmenan, p. 23. 
RoE, E. M. F. (1956) Applications of the reflecting microscope. Modern Methods of Microscopy. Butterworths, p. 23.

SagnaC, M. (1911) Strioscopie interférentielle. J. "le radium" 8, 251.

SAYlor, Ch. P., BRICE, A. T. and ZERNIKE, F. (1950) Colour phase-contrast microscopy, requirements and applications. J. Opt. Soc. Amer. 40, 329.

Schmaltz, G. (1936) Technische Oberflächenkunde. Berlin.

Sмiтh, F. H. (1956) Microscopic interferometry. Modern Methods of Microscopy. Butterworths, p. 76.

SteEl, W. H. (1957) The design of reflecting microscope objectives. Aust. J. Sci. Res. 4, 1. SVENSSON, G. (1957) Scanning interference microphotometry. Cytochemical Methods with Quantitative Aims. Academic Press, N. Y., p. 165.

TAYLOR, E. W. (1950) The application of phase-contrast to the ultraviolet microscope. Proc. Roy. Soc. 137, 332.

ThORELL, B. and AKERMANN, L. (1957) Recording microspectroscopy for the study of intracellular reactions. Cytochemical Methods with Quantitative Aims, Academic Press, N. Y., p. 83.

TOLANSKY, S. (1948) Multiple Beam Interferometry. Clarendon Press, Oxford.

TOlansky, S. (1955) Microstructures of Diamond Surfaces. N.A.G. Press, London. TORIKAI, Y. (1952) On the image formation in phase microscopy. J. Phys. Soc.Japan 7, 330. VÄISÄlÄ, Y. (1922/23) Ann. Univ. åbo (turku.) A 1 No. 2, 131.

WALKER, P. M. B. (1958) Ultraviolet microspectrophotometry. General Cytochemical Methods. Academic Press, N. Y., p. 163.

Wilkins, M. A. F. and Norris, K. P. (1952) An easily-used ultraviolet microscope objective. Nature, Lond. 170, 883.

Wolter, H. (1950) Zur Dentung von Beobachtungen mit dem phasenkontrastverfahen, Naturwissenschaften, 37, 272.

Wolter, H. (1950) Farbige Phasenkontrastverfahren. Naturwissenschaften 37, 491.

ZERNIKE, F. (1934) Bengungstheorie des schneidenverfahrens und seiner verbesserten form, der phasenkontrastmethode. Physica 1, 689.

ZERNIKE, F. (1946) Phase-contrast a new method for microscopic observation of transparent objects. Achievements in Optics. Elsevier Publisher, Amsterdam, p. 116. 


\section{INDEX}

Airy disk 6

Annular-diaphragm (phase microscope) 77

Anoptral device (Reichert) 79

Area edge (incoherent illumination) 36

Astigmatism 22

Axial settings (role of the observers`s eye) 165

Axial setting accuracy 164

Axial image duplication method (interference microscopy) 109

Basic system for microspzctrophotometry with photo-cell 247

Bausch and Lomb interference microscope 153

Bausch and Lomb ultra-violet microscope 239

Bendford and Seidenberg device (phase contrast) 136

Birefringence (measurement) 210

Black line (coherent a partially coherent illumination) 42

Black line in coherent illumination 355

Blaisse's interference microscope 121

Bouwers and Blaisse solid reflecting objective 235

Burch microscope objectives 230-238

Calibrating the hot stage 270

Caspersson microspectrophotometric equipment 250

Cassegrain type reflecting objective 229

Chapman and Alldridge device 140

Characterizing and identifying fusible compounds 284

Chemical Microscopy 267

Chromatic effects on the image contrast 58

Chromatism produced by diffraction 15

Coated surface 60

Coherent light 26

Colour phase-contrast 88

Coma $21^{\circ}$

Compensated interference eyepiece 116

Complete image duplication method 105

Condenser aperture (effect on the resolution of two pin-points) 55

Contact angle measurement 222

Contact method (Köfler) 274

Contrast 33

Contrast factor of an optical instrument 39

Contrast image produced by the object 73

Cooke, troughton and Simms photometer eyepiece 262

Critical illumination 28 
Dark-ground in reflected light 136

Dephasing object 64

Depth of focus produced by chromatism 14

Determination of eutectic point composition 281

Determining the melting point 271

Determining the refraction index from path-difference measurement 179, 205

Determining the refraction index from path-difference measurement (Full-duplication process) 195

Diagram (binary mixtures) 279

Diagram (temperature-composition) 276

Differential method (interference microscopy) 107

Diffracted light in the focal plane of a microscope objective 57

Diffraction by parallel slits 44

Diffraction fringes surrounding a focal line 23

Diffraction pattern 3

Diffraction pattern (apochromatic, fluorite and achromatic objective) 18

Diffraction pattern in presence of coma 22

Diffraction pattern (isophotes) 13

Diffraction pattern (isophotes in presence of spherical aberration) 20

Diffraction pattern (isophotes when spherical aberration coma and astigmatism are present) 24

Diffraction patterns of various numerical apertures 7

Diffraction patterns when focusing is altered 12

Diffraction pattern with a reflecting objective 9

Double beam arrangement with photo-cell 248

Double beam microspectrophometric device 244

Dry masses (measurement) 226

Duplicated waves 106

Dysons's interference microscope (transmitted light) 97

Dyson interference microscope (reflected light) 147

Dyson long working distance attachment 161

Edge of area (coherent and partially coherent illumination) 43

Effect of the condenser aperture on the resolution of a periodic object 55

Effect of the condenser aperture on the resolution of two pin-points 55

Errors in microspectrophotometry 258

Eutectic composition (Köfler method) 280

Eutectic (index measurement) 209

Extended object (coherent illumination) 40

Extended objects (incoherent illumination) 31

Eyepiece with illuminated reticle 170

Field curvature 22

Fluorescence 239

Fluorescence spectrophotometry 263

Foster device 160

Foucault test 37

Francon's compensated interference eyepiece 113

Half-sphere (phase contrast) 87

Interference microscope (axial duplication method) 121

Interference microscope (reflected light) 148 
Francon and Nomarski phase contrast device (reflected light) 135

Francon-Nomarski phase contrast device (transmitted light) 84

Fringe-shift path-difference measurements (differential process) 203

Fusible compounds (characterizing and identifying) 284

Geometrical measurements 163

Gibson and Tyndall curve 15

Grain of photographic images 61

Grey and Lee reflecting objective 233

Half-plane object 37

Hartley phase-plate 83

Heine's condenser 80

Heine phase contrast microscope $8 \mathrm{i}$

High precision-measurement microscopes 172

Hilger and Watt interference microscope 154

Hot-metal surfaces 161

Hot stage 267

Illuminated half-plane object 36

Illumination of objects 24

Image contrast 33

Image contrast (effect of chromatism) 58

Image contrast in phase microscopy 71

Image converter 236

lmage duplication in a polarization interferometer 104

Image formation (extended object) 31

lmage formation (incoherent extended object) 32

lmage of a luminous point in monochromatic light 2

Image of a luminous point in white light 14

Image of a luminous point originated by a reflecting objective 8

Image of a luminous point when the microscope is not properly focused 9

Image of a periodic object in coherent illumination 44

Image of a small black disk (coherent illumination) 33

Image of a small white disk 34

Image of bright small disk (black ground) 42

Image of thin black line (coherent or partially coherent illumination) 42

Image of thin black line (coherent illumination) 43

Image structure in phase-contrast 73

Incoherent light 27

lndex measurement of a melted eutectic 209

Infra-red microscopy 228

Infra-red phase contrast 89

Interference eyepiece 115

Interference microscope applied to micro-hardness tests 154

Interference microscope (measurement accuracy) 218

Interference microscope (principals) 95

Interference microscope (sensitivity) 128

Interference microscopy (axial image duplication method) 109

Interference microscopy (complete image duplication) 105

Interference microscopy (differential method) 107 
Interference microscopy (transmitted light) 94

Jamin interferometer 103

Johansson's interferometer eyepiece 117

Johnson reflecting objective 232

Jupnik, Osterberg and Pride phase microscope 134

Kastler and Montarnal phase-plate 82

Köfler contact method 274

Köfler hot stage 268

Köfler method for determination of eutectic composition $\quad \mathbf{2 8 0}$

Köhler illumination 28

Köhler illumination in reflected light 130

Koristka high precision-measurement microscope 172

Krug and Lau interference microscope 146

Lateral setting 164

Lebedeff interference microscope 104

Leitz's interference microscope 101

Leitz's microscope-photometer 261

Leitz's ultropak 141

Lieberkuhn device 140

Light distribution in the Foucault test 37

Linnik's interference microscope 143

Locquins's phase-plate 84

Long working distance attachment (Dyson) 161

Luminous point in the presence of aberrations 19

Luminous points on black ground (coherent illumination) 54

Luminous points on black ground (incoherent illumination) 53

Maksutov reflecting objective 232

Maksutov solid reflecting objective 234

Measurement accuracy with an interference microscope 218

Measurements (microspectrophotometry) 255

Measurements with interference fringes 125

Measuring a length by means of lateral and axial settings 168

Measuring a length by means of two lateral settings 167

Measuring a thin object by 2 axial settings 168

Measuring angles of contact between liquid and solid surfaces 222

Measuring birefringence through a polarizing interference microscope 210

Measuring dry masses by means of an interference microscope 226

Measuring path differences 174

Measuring path differences, applying the fringes process (full-duplication process) 187

Measuring path differences by means of the flat-tints process (full-duplication process) 181

Measuring path difference using the flat-tints method (differential process) 199

Measuring refraction indices of transparent objects polarizing interference microscopes 181

Measuring refraction indices of transparent objects (unpolarized two-waves interference microscopes) 174

Measuring slopes with interference microscope 212

Measuring small areas and volumes 169

Measuring the refraction index of a melted substance 273 
Measuring the refraction index of liquids (differential process) 206

Measuring the refraction index of liquids (full-duplication process) 197

Measuring the surface slopes 142

Measuring thickness with interference microscope 212

Melting point (determination of) 213, 215, 218, 271

Menzel's interference microscope 127

Menzel's method (reflected light) 139

Microscope (high precision-measurement) 172

Microspectrophotometric measurements 255

Microscope objectives (ultra-violet and infra-red) 228

Microspectrophotometry 242

Microspectrophotometry (basic system with photo-cell) 247

Microspectrophotometry (double beam device) 244

Microspectrophotometry (errors) 258

Microspectrophotometry (fluorescence) 263

Microspectroscopy 242

Mirau interference microscope 146

Mixed fusion 274

Multi-wave interference microscopes 122

Newton's colour scale 185

Nomarski and Bernstein reflecting objective 234

Nomarski interference microscope (reflected light) 149

Nomarski quartz plate device 131

Nomarski's interference microscope (transmitted light) 118

Normal in a point of a small reflecting surface 169

Norris and Wilkins solid reflecting objective 235

Object illuminated in monochromatiz light (microspectroscopy) 243

Object illuminated in white light (microspectroscopy) 254

Oblique illumination 48

Oblique illumination in reflected light 136

Optical filtering of a photographic image 61

Optimun magnification 34

Optique Précision de Levallois hot stage 270

Osterberg's polanret microscope 82

Path difference (measurement) 174

Path difference (measurement by full-duplication process) 181

Path difference (measurement by the fringes process) 187

Path difference (measurement with the differential process) 199

Path difference (measurement with the flat-tints method) 199

Path difference produced by a shift of the focusing plane 10

Payne's device (phase contrast) 86

Peltier-effect applications to hot stage 269

Perception limit 48

Perception limit (effects of stray light) 75

Perception limit of a perfect instrument (numerical values) 58

Periodic object (coherent and partially coherent illumination) 43

Periodic object (coherent illumination) 44

Periodic object in incoherent illumination 37 
Periodic object in oblique illumination 48

Phase-contrast applications 90

Phase contrast (image-contrast) 71

Phase contrast (image structure) 73

Phase contrast (infra-rouge) 89

Phase contrast microscope 77

Phase contrast microscopy 64

Phase contrast (reflected light) 133

Phase contrast (sensitivity) 71

Phase contrast (ultra-violet) 89

Phase plate 69

Photometric device using a plastic-material rod 263

Photometric eyepieces 260

Polanret microscope 82

Polarization interference microscope 103

Polarized light (by reflection) 158

Range of coherence 27

Range of coherence with an extended source 27

Rayleigh's law 20

Recording infra-red imagery 236

Recording ultra-violet imagery 236

Reflectance of a surface 60

Reflected-light microscopy 129

Reflected-light phase-contrast 133

Reflecting objectives 228

Refraction index (liquids) 197-206

Refraction index (measurement of a melted substance) 273

Refraction indices (determining from path difference measurement) 179

Refraction indices (measurement with polarizing interferences microscope) 181

Refraction indices (measurement with unpolarized two-waves interterence microscope) 144

Reichert's anoptral device 79

Reichert's device (Epilum) 141

Reichert hot stage microscope 269

Replica method 157

Resolving power 48

Resolving power (effects of stray light) 75

Resolving power (incoherent illumination) 49

Resolving power of a perfect instrument (numerical values) 58

Sagnac's interference microscope 142

Savart plate 114

Schmaltz's slit 136

Schmaltz's slit (improved method) 138

Scharzschild-type reflecting objective 229

Sensitivity of interference microscopes 128

Sensitivity of phase contrast method 71

Setting accuracy 163

Setting in microscopy 163

Slopes (measurement) 212 
Small black disk (coherent illumination) 33

Small black disk (incoherent and partially coherent illumination) 40

Small black disk in relation to the aperture condenser 41

Smith's interference microscope 110-120

Solid image converter 237

Spectral distribution of the photometrists standard $A \quad 17$

Spherical aberration 19

Stray light 59

Stray light reflected by lenses of an objective 131

Structure of small objects 50

Taylor phase-plate 84

Temperature-composition diagram 276

Temperature-composition diagram of binary mixtures 279

Thickness (measurement) 212-213, 215, 218

Thin film 60

Thomas Merton interference microscope 126

Thorell arrangement (microspectroscopy) 249

Thornburg reflecting objective 231

Transparent objects 64

Transverse setting accuracy 163

Two-wave interference microscopes 94

Ultra-violet phase contrast 89

Ultra-violet microscopy 228

Water surface deformation produced by a small insect 225

Wollaston prism 110

Young's experiment 25

Zeiss interference microscope 144

Zeiss quarter-wave device 131

Zeiss ultra-violet objectives 235

Zernike phase plate 78

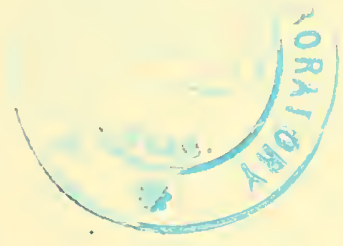




